\title{
Review of the Decomposition of Ammonia to Generate Hydrogen
}

\author{
Ilaria Lucentini, Xènia Garcia, Xavier Vendrell, and Jordi Llorca* \\ Cite This: https://doi.org/10.1021/acs.iecr.1c00843 \\ Read Online
}

ACCESS |

Wlll Metrics \& More

回 Article Recommendations

ABSTRACT: Because of the problems associated with the generation and storage of hydrogen in portable applications, the use of ammonia has been proposed for on-site production of hydrogen through ammonia decomposition. First, an analysis of the existing systems for ammonia decomposition and the challenges for this technology are presented. Then, the state of the art of the catalysts used to date for ammonia decomposition is described considering the catalysts composed of noble and non-noble metals and their combinations, as well as novel materials such as alkali metal amides and imides. The effect of the supports and promoters used is analyzed in detail, and the catalytic activity obtained is compared. An analysis of the kinetics of the reaction obtained with different catalysts is also presented and discussed, including the reaction mechanism, the determining step of the reaction, and the apparent activation energy. Finally, the structured reactors used to date for the decomposition reaction of ammonia are explored, as well as the possibilities offered by catalytic membrane reactors, which allow the on-site simultaneous production and separation of hydrogen.

\section{INTRODUCTION}

The fundamental problem of the current energy system is the great negative environmental impact of energy production using fossil fuels. ${ }^{1}$ Alternative technologies to fossil fuels have been added over time and existing technologies have been improved; however, a radical change in the energy system, the so-called "energy transition", has yet to take place. In the framework of the energy transition toward a new clean and efficient system, the dramatic decrease in costs in solar and wind technologies, and now in energy storage technologies, is a strong impulse toward the radical change that is proposed. ${ }^{2}$ It should be pointed out that the energy transition is not only technological, but also a combination of economic, political, institutional, and sociocultural changes, and it must be based on ethics and sustainability. ${ }^{3}$

1.1. Hydrogen as an Energy Vector. The "hydrogen economy" is a concept introduced a long time ago. This term was used by John Bockris in 1972, and proposes to base the energy transition on the use of hydrogen as a vector for the generation of clean and environmentally sustainable energy. ${ }^{4}$ In recent decades, the production of hydrogen from various sources, its transport and storage, and finally its use to provide energy with low emissions have been extensively investigated. ${ }^{5}$ Hydrogen is taking one of the leading positions in the energy sector for stationary and transport applications, including vehicles and other means of transport, auxiliary power units, stationary power generation in domestic and industrial applications, and as an energy vector to store the excess of electrical energy generated off-peak. ${ }^{6}$
Nowadays almost all the hydrogen produced comes from catalytic steam reforming of fossil fuels, mainly from natural gas, ${ }^{7,8}$ which is currently a well-established commercial technology and is the least expensive way to produce hydrogen on a large scale. Although the electrolysis of water is a wellknown and established technology to produce clean and high purity hydrogen, ${ }^{9}$ it comprises high energy losses. ${ }^{10}$ Nevertheless, a large reduction in the cost of electricity from renewable sources and electrolyzers is needed to allow the hydrogen produced by electrolysis to compete with conventional sources of energy on a large scale. ${ }^{11}$ Currently, most of the hydrogen is produced and used on-site in industry, principally for ammonia production and petroleum refining, which together account for two-thirds of the total hydrogen use. ${ }^{12}$ Hydrogen represents an effective and clean alternative to the use of fossils fuels, which have a high carbon footprint, to produce energy by using fuel cells, since water is the only product generated in the process, apart from heat. Moreover, fuel cells have the ability to continuously produce electricity while fueling them, which is a significant advantage compared to batteries. Currently, there are several commercialized fuel cell-based technologies that differ mainly in the electrolyte, fuel and/or working temperature. The

Special Issue: José Luis García Fierro Festschrift

Received: March 2, 2021

Revised: May 10, 2021

Accepted: May 11, 2021 
most common fuel cells are solid oxide fuel cells (SOFC), proton exchange membrane (PEMFC), direct methanol (DMFC), alkaline (AFC), phosphoric acid (PAFC), and molten carbonate (MCFC) fuel cells. PEMFCs are the most widely used for mobility and small applications. ${ }^{13}$

Another of the current challenges in hydrogen technologies is its storage and transport. Hydrogen has a very high energy density by mass $\left(119.7 \mathrm{MJ} \mathrm{kg}^{-1}\right.$ of lower heating value at $25^{\circ} \mathrm{C}$ and $\left.1 \mathrm{bar}^{14}\right)$, but it has a very low energy density by volume $\left(8.96 \mathrm{GJ} \mathrm{m}^{-3}\right.$, referred to as liquid fuel $\left.{ }^{15}\right)$. Furthermore, hydrogen tends to diffuse through the materials, leading to embrittlement or weakening of the storage material. ${ }^{16}$ Nowadays, hydrogen is commonly stored as compressed gas at pressures up to 700 bar at $25{ }^{\circ} \mathrm{C}$. ${ }^{17}$ It can be also stored as a liquid with a higher volumetric energy density at much lower temperatures $\left(-253{ }^{\circ} \mathrm{C}\right.$ at 1 bar $) .{ }^{18}$ The cryo-compression, where hydrogen is cooled down until the pressure required for its compression drops to $350 \mathrm{bar},{ }^{19}$ offers an alternative to store hydrogen, although the energy needed is very high. ${ }^{20}$ Alternatively, hydrogen can be adsorbed on materials with a large surface area and pores of adequate size (such as carbonaceous materials or carbon nanotubes), ${ }^{21,22}$ metal hydrides, ${ }^{23}$ and structures based on metal-organic frameworks (MOFs). ${ }^{24,25}$ However, the adsorption/absorption of hydrogen leads to an increase in its transportation weight and volume ${ }^{26}$ and there are difficulties in the regeneration processes of the materials. ${ }^{23}$ A different approach is the chemical storage in the form of another hydrogen-containing compound that can be easily transported, and hydrogen is generated on-site through a chemical reaction. ${ }^{27}$ In this sense, the chemical storage can be in the form of simple hydrides like $\mathrm{LiH}, \mathrm{NaH}, \mathrm{KH}$, or $\mathrm{CaH}_{2}$, and also as binary hydrides such as $\mathrm{LiBH}_{4}, \mathrm{NaBH}_{4}, \mathrm{KBH}_{4}, \mathrm{LiAlH}_{4}$, or $\mathrm{NaAlH}_{4}{ }^{28}$ However, these compounds must be synthesized from the respective metals and hydrogen, which involves a high loss of energy. ${ }^{29,30}$ For example, in the case of calcium hydride, losses are at least $60 \%$, and for other compounds the losses can be even higher. ${ }^{28}$ For this reason, up to date, the chemical storage of hydrogen in these types of compounds has a limited practical application. Nevertheless, other interesting compounds for chemical storage are synthetic fuels that are easy to synthesize and can be stored in a liquid form at ambient or near-ambient conditions; one of the most promising compounds is ammonia. ${ }^{31}$

1.2. Ammonia as a Source of Hydrogen. Ammonia has a high hydrogen content $(17.8 \%$ by weight and a volumetric density of $121 \mathrm{~kg} \mathrm{H}_{2} \mathrm{~m}^{-3}$ at $\left.10 \mathrm{bar}\right)^{32}$ and can liquefy at low pressure, 8.6 bar at $20{ }^{\circ} \mathrm{C},{ }^{33}$ so its transport and storage are relatively easy and require a low amount of energy. ${ }^{34,35}$ The decomposition reaction of ammonia is endothermic $\left(2 \mathrm{NH}_{3(\mathrm{~g})}\right.$ $\left.\leftrightarrows \mathrm{N}_{2(\mathrm{~g})}+3 \mathrm{H}_{2(\mathrm{~g})} ; \Delta H^{\circ}=92 \mathrm{~kJ} \mathrm{~mol}^{-1}\right)$ and reaches $99.99 \%$ ammonia conversion at $400{ }^{\circ} \mathrm{C}$ and 1 atm according to thermodynamics, considering an inlet flow composed only of ammonia. This means that a moderately high operating temperature is required to drive the ammonia decomposition reaction to completion and thus produce very high purity hydrogen. This purity is compulsory if the hydrogen produced is used in fuel cells such as PEMFCs, which are irreparably degraded at very low concentrations of ammonia (ca. 0.1 $\mathrm{ppm}) .^{32}$ Alternatively, hydrogen-selective membrane systems can be used, such as catalytic membrane reactors. ${ }^{36}$

Ammonia is the second most widely produced chemical, after sulfuric acid, ${ }^{37}$ and is mainly used in fertilizer manufacturing, which is the largest source of demand ( $88 \%$ of the total ammonia produced). ${ }^{38}$ More than $90 \%$ of the total production of ammonia is carried out by the inverse reaction of decomposition, which is the so-called Haber-Bosch process, originally developed by Fritz Haber and Carl Bosch. Iron catalysts with promoters, temperatures around $400-600{ }^{\circ} \mathrm{C}$, and pressures between 100 and 400 bar are typically used. Starting in 1990, ruthenium was introduced as a catalyst, which has allowed the reaction pressure to be lowered, but due to its high cost, it is only used in a few plants. $^{39}$ In the synthesis of ammonia, the production of the mixture of $\mathrm{H}_{2}$ and $\mathrm{N}_{2}$ has the greatest contribution to the total cost of the process; moreover, energy from fossil fuels is used almost exclusively for the production of $\mathrm{H}_{2}$ (steam reforming of natural gas) and the separation of $\mathrm{N}_{2}$ from air. ${ }^{38}$ Ammonia production consumes about $2 \%$ of the world's energy supply and its production releases more than 400 Mt of $\mathrm{CO}_{2}$, which represents $1.6 \%$ of the total global emissions. ${ }^{40}$ Therefore, several attempts have been made to decarbonize the $\mathrm{NH}_{3}$ production process through the concepts of "blue ammonia" production using carbon capture systems, and "green ammonia" using hydrogen produced from electrolysis of water. ${ }^{41}$

Currently, the ammonia decomposition reaction is applied industrially mainly for annealing metals and galvanizing. The ammonia crackers use external energy sources, nickel supported on aluminum oxide as catalyst, and operate at a temperature of about $850-950{ }^{\circ} \mathrm{C}$. ${ }^{42}$ To apply ammonia decomposition in power generation (ammonia-to-power) and to minimize the presence of ammonia in the outlet gas, several solutions have been proposed:

- First, the ammonia can be separated from the stream by cooling. The fraction of condensed ammonia depends on the pressure of the gas stream, and at low pressures, such as atmospheric pressure, the required temperature is below zero. ${ }^{43}$ To avoid lowering the gas temperature, ammonia capture materials, such as $\mathrm{CaCl}_{2}, \mathrm{MgCl}_{2}, \mathrm{MgBr}_{2}$, or $\mathrm{CaBr}_{2}$ have been used on porous supports, silica or zeolite, which can remove ammonia at high temperatures (typically $100-250{ }^{\circ} \mathrm{C}$ and 5-30 bar). These materials can emit less than $0.1 \mathrm{ppm}$ of ammonia from an input concentration greater than $10000 \mathrm{ppm}$. The main disadvantage of using these materials is that the ammonia cannot be directly recirculated in the process, but has to be desorbed from the material at temperatures around $300-400{ }^{\circ} \mathrm{C}$. Furthermore, these materials do not adsorb nitrogen, and if the output gas is used to supply $\mathrm{H}_{2}$ to a PEM-type fuel cell in mobile applications, such as in a vehicle, the requirement of maximum $100 \mathrm{ppmv} \mathrm{N}_{2}$ has to be met, necessary so that the inlet stream to the PEMFC has a purity of $\mathrm{H}_{2}$ greater than $99.97 \%$ (ISO 14687 2:2012). ${ }^{44}$ Currently these technologies are on a laboratory scale or are used in pilot plants. ${ }^{43}$

- Second, alternative fuel cells that are not affected by high levels of ammonia in the hydrogen stream could be used. In this sense AFCs can tolerate ammonia concentrations of up to $9 \% .{ }^{45}$ However, the main limitation to the direct use of ammonia is that at the low operation temperatures of AFCs, ammonia does not decompose easily, and therefore an external reformer is necessary. Recently, high temperature PEMFCs have shown a greater capacity to resist poisoning by other compounds such as $\mathrm{CO}$, and they have also the potential to resist higher concentrations of ammonia. ${ }^{46}$ In SOFCs, ammonia can be used directly 


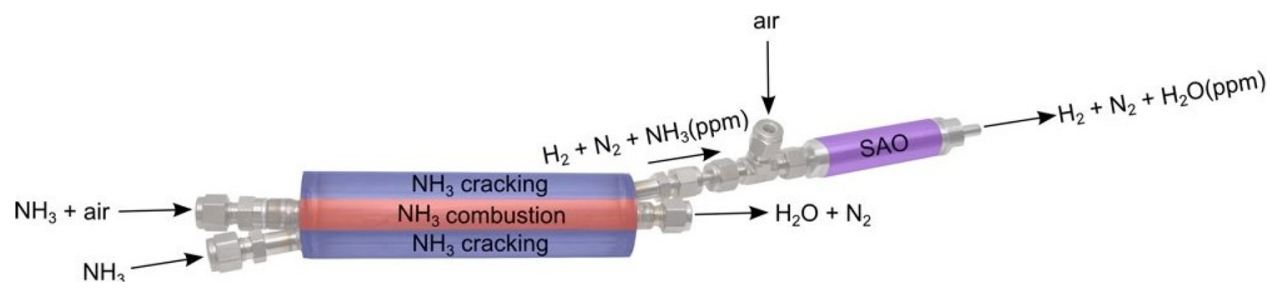

Figure 1. Scheme of the system used by Rencat. ${ }^{65}$ Reprinted with permission from RenCat (https://rencat.net/).

as fuel at high temperatures, at which the decomposition occurs directly at the anode. ${ }^{36}$ Ammonia-fed SOFCs have been tested at pilot scale at Kyoto University, Japan, in 1 $\mathrm{kW}$ applications. ${ }^{47}$

- Third, ammonia can be used directly as a fuel in gas turbines or engines, exploiting its high octane number $(110-130) .^{48}$ The main obstacle of these technologies is the emission of $\mathrm{NO}_{x}$ associated with the combustion of ammonia. Ammonia in a gas turbine has initially been used in the form of mixtures with air ${ }^{47}$ or with water steam, ${ }^{49}$ as well as coupled with other fossil fuels, which leads to a reduction in carbon emissions without losing energy efficiency. Gas turbines fired directly with ammonia have been tested in pilot plants in Japan (IHI Corporation). ${ }^{50}$ However, the application of ammonia as an engine fuel in a vehicle is still in the prototype stage. In this sense, two options have been examined: the direct combustion of ammonia alone or in mixtures, or the decomposition and use of the $\mathrm{H}_{2}$ produced on-board as fuel. In 1905, the first ammonia combustion engine was developed by Ammonia Casale Ltd.., which was patented in Italy in $1935-36 .^{51}$ Later on, in 1933, Norsk Hydro built a prototype vehicle with a hydrogen combustion engine, produced from the decomposition of ammonia. ${ }^{52}$ Another approach for using ammonia in a combustion engine is mixing it with hydrogen; ammonia mixtures containing a minimum of $10 \%$ by volume hydrogen have been shown to be very effective. ${ }^{32}$ In 2013 , a hybrid car prototype was presented, the Marangoni Toyota GT86-R Eco Explorer, which worked with a mixture of $\mathrm{NH}_{3}-\mathrm{H}_{2}$, for which the $\mathrm{H}_{2}$ came from the decomposition of the same $\mathrm{NH}_{3}$ in a separate catalytic reactor using the heat of the exhaust gases. ${ }^{53}$ Apollo Energy Systems (AES) ${ }^{54}$ and Intelligent Energy Corporation (IE) ${ }^{55}$ have patented ammonia decomposition systems to supply hydrogen at a PEMFC of $\sim 10 \mathrm{~kW}$ for vehicle applications. ${ }^{37}$ Pochari Technologies has designed a system to decompose ammonia in a microchannel reactor to supply $\mathrm{H}_{2}$ to a PEMFC in vehicles. ${ }^{56}$

Concerning medium-sized systems for stationary power generation applications, different companies have designed systems that use ammonia for power generation. In this sense, Tower Power uses the generated energy to power cell phone towers in locations that lack a consolidated electricity service. In 1999 they built the first prototype and in 2012 the first equipment was installed in Namibia to generate a power of 1.2 kW. Currently, eight different updated models of ammonia crackers (ToWER/CuBE) with an output power between 1.3 and $10 \mathrm{~kW}$ are available. ${ }^{57}$ Another company, GenCell, has recently developed a system (GenCell A5) that can produce electricity $(4 \mathrm{~kW})$ from the decomposition of ammonia continuously for a year disconnected from the electricity grid. The system is already in the commercial phase and has a lower cost than a diesel system of the same dimensions. ${ }^{58}$ In a different way, the company AFC Energy has tested an ammonia decomposition system connected to an AFC of $240 \mathrm{~kW}$, which has successfully completed an 18-month field test. ${ }^{59}$ In United Kingdom, the Science and Technology Installations Council (STFC), which had already developed a prototype for the generation of energy in vehicles from the decomposition of ammonia contained in amides, ${ }^{60}$ together with Siemens, Ecuity, and Engie, has started a project to supply low-cost stationary energy from $\mathrm{H}_{2}$ produced from the decomposition of $\mathrm{NH}_{3} \cdot{ }^{61,62}$

Regarding portable applications of very small dimensions (between 50 and $150 \mathrm{~W}$ ) there are projects at prototype scale or in the first steps for their commercialization. The compact system developed by Meso Systems Technology, Inc. (MTI) uses a microchannel reactor (MesoChannel) to decompose ammonia and produce $50 \mathrm{~W}$ integrating it to a PEMFC. ${ }^{63}$ In a similar way, Analytic Power Corporation uses ammonia decomposition (A-Cracker) to provide hydrogen for small power supplies using $150 \mathrm{~W}$ fuel cells. ${ }^{64} \mathrm{~A}$ new company in Denmark (Rencat) commercializes a technology to generate low-cost, high-purity hydrogen from ammonia for use in fuel cells (Figure 1, RenGen) that uses catalytic decomposition and oxidation of ammonia simultaneously. ${ }^{65}$ Other organizations such as CSIRO in Australia ${ }^{66}$ and Bettergy Corp. in the USA ${ }^{67}$ develop ammonia decomposition systems to generate energy using hydrogen-selective membrane reactors.

\section{HYDROGEN GENERATION FROM AMMONIA}

The decomposition of ammonia occurs at high temperature in the presence or absence of a catalyst. One of the first works about the decomposition of ammonia reaction was carried out in 1904 by Perman and Atkinson. ${ }^{68}$ The effect of temperature and pressure on the decomposition rate was evaluated, as well as the catalytic activity of elements such as $\mathrm{Hg}, \mathrm{Fe}$, and Pt. Over time, the decomposition of ammonia has proven to be an interesting reaction for different industrial applications; in 1934 Tyler $^{69}$ proposed the use of the hydrogen produced through the decomposition of ammonia at high pressures (7-14 bar) coupled with a residual ammonia scrubber to harden oils. It is important to mention that the technology of ammonia crackers at ambient pressure was already established in the metallurgical industry to reduce and temper metals. Regarding the effect of pressure, the decomposition of ammonia is favored at low pressures, for this reason many studies have focused on investigating the reaction rate at low pressures up to ultrahigh vacuum in the presence of platinum, ${ }^{70}$ nickel, rhodium, tantalum, tungsten, ${ }^{71}$ and iridium ${ }^{72}$ catalysts. More recently, the effect of high pressures on the reaction rate was examined, considering that generally the hydrogen produced has to be compressed for its supply, for example, to a fuel cell. ${ }^{73}$ In this sense, in order to avoid compressing the hydrogen generated, the decomposition of ammonia has been evaluated directly at high pressures, up to $40 \mathrm{bar}$, in the presence of a $\mathrm{Ru} / \mathrm{CaO}$ 
catalyst promoted with $\mathrm{K} .{ }^{73}$ Di Carlo et al. ${ }^{74}$ tested a $\mathrm{Ru} / \mathrm{Al}_{2} \mathrm{O}_{3}$ catalyst at pressures between 1 and $10 \mathrm{bar}$, evaluating the decrease in conversion with increasing pressure.

As a result of the first studies and applications, and in parallel with the research of catalysts for thermal decomposition, alternative methods have been proposed to provide the activation energy necessary for the reaction, among which is the application of electric current, electron beams or ions, microwave, plasma, or solar energy. Integrated systems have also been studied in which the ammonia decomposition has been coupled with other parallel exothermic reactions, such as the combustion of propane or butane. These technologies can be applied with or without the presence of catalysts. The production of hydrogen from the electrolysis of liquid ammonia has also been studied, by photocatalysis, mechanochemical methods, or the decomposition of ammonia in the presence of other compounds such as hydrocarbons, $\mathrm{H}_{2} \mathrm{~S}$, oxygen or water. Some of these methods have been proposed to avoid unwanted ammonia emissions. The different technologies that have been proposed in the literature are listed in Table 1.

Table 1. Technologies Used to Decompose Ammonia for Removal or for the Production of Hydrogen

\begin{tabular}{|c|c|c|}
\hline technology & year & ref \\
\hline thermal decomposition & 1904,1934 & 68,69 \\
\hline $\begin{array}{l}\text { decomposition at } \\
\text { pressures other than } \\
1 \text { bar }\end{array}$ & 1967, 1968, 2001, 2020, 2014 & $70-74$ \\
\hline $\begin{array}{l}\text { decomposition with } \\
\text { electric current }\end{array}$ & 1997, 2000, 2002, 1938, 2013 & $75-79$ \\
\hline $\begin{array}{l}\text { decomposition with an } \\
\text { electron beam }\end{array}$ & 1928, 1980, 1970, 2013 & $80-83$ \\
\hline $\begin{array}{l}\text { decomposition with an ion } \\
\text { beam }\end{array}$ & 2016 & 84 \\
\hline microwave decomposition & $2017,2017,1972$ & $85-87$ \\
\hline $\begin{array}{l}\text { decomposition with } \\
\text { plasma technologies }\end{array}$ & $\begin{array}{l}1967,2013,2019,2019,2014,2017 \\
2018,2015\end{array}$ & $88-95$ \\
\hline $\begin{array}{l}\text { decomposition with solar } \\
\text { energy }\end{array}$ & 2020,2019 & 96,97 \\
\hline $\begin{array}{l}\text { decomposition coupled } \\
\text { with other reactions }\end{array}$ & $\begin{array}{l}2017,2018,2017,2012,2011,2003 \\
\quad 2009,2013,2005\end{array}$ & $98-106$ \\
\hline electrolysis of liquid $\mathrm{NH}_{3}$ & 2010,2016 & 107,108 \\
\hline $\begin{array}{l}\text { photocatalysis in gaseous } \\
\text { or aqueous medium }\end{array}$ & $2015,2018,1932,1983,2012$ & $109-113$ \\
\hline $\begin{array}{l}\text { decomposition with } \\
\text { mechanochemical } \\
\text { methods }\end{array}$ & 2010 & 114 \\
\hline $\begin{array}{l}\text { reaction of } \mathrm{NH}_{3} \text { with } \\
\text { hydrides }\end{array}$ & 2007 & 115 \\
\hline $\begin{array}{l}\text { decomposition in } \\
\text { gasification atmospheres }\end{array}$ & $\begin{array}{l}1905,1996,1999,1995,1997,2008 \\
2008,2004,1993,1995,2002,2002\end{array}$ & $116-127$ \\
\hline $\begin{array}{l}\text { decomposition in the } \\
\text { presence of } \mathrm{H}_{2} \mathrm{~S}\end{array}$ & $2008,2005,2000,2002$ & $128-131$ \\
\hline $\begin{array}{l}\text { decomposition in the } \\
\text { presence of oxygen }\end{array}$ & $2012,2008,2015,2002,2017$ & $132-136$ \\
\hline $\begin{array}{l}\text { decomposition in } \\
\text { wastewater }\end{array}$ & 1999 & 137 \\
\hline $\begin{array}{l}\text { decomposition in the } \\
\text { presence of water vapor }\end{array}$ & 1977,2014 & 138,139 \\
\hline
\end{tabular}

2.1. Energy Supply Methods. The first experiments related to the decomposition of ammonia using electric current were focused on studying the decomposition as the inverse reaction of ammonia synthesis. The first tests were carried out in one or two chamber cell reactors made up of ceramic materials with $\mathrm{Fe} / \mathrm{K}^{+},{ }^{75} \mathrm{Pd}^{76}$, and $\mathrm{Ag}^{77}$ electrodes, obtaining ammonia conversions between 25 and $35 \%$ at temperatures of 500-600
${ }^{\circ} \mathrm{C}$. In the case of $\mathrm{Fe} / \mathrm{K}^{+}$electrodes, poisoning by ammonia was observed. ${ }^{75}$ In these cells, the temperature at which ammonia begins to decompose remains very high, while if a strong electric field is coupled with alpha particles, the reaction temperature can be significantly lowered. In the experiments reported by Smith and Essex in $1938,{ }^{78}$ Pt electrodes were used, and it was demonstrated that the decomposition of ammonia was mainly due to alpha particles. After these first works, the studies were extended until reaching conversions of ammonia comparable to those obtained with thermal decomposition. In this sense, Zhao et al. $^{79}$ analyzed the results in an alternating current discharge reactor testing three types of electrodes $(\mathrm{Cu}$, stainless steel, and $\mathrm{Ni}$ ), obtaining an almost complete conversion with $\mathrm{Ni}$ electrodes. Other attempts to decompose ammonia by eliminating the effect of temperature was carried out in 1928 by McLennan and Greenwood, ${ }^{80}$ who investigated the ammonia decomposition in a cathode ray tube. In their study, it was initially determined that the electric discharge generated in the reactor completely decomposed the ammonia in a very short time, and that the chamber composed of glass had an initial catalytic effect. By eliminating the generation of electric current, they analyzed the decomposition due only to the high-speed electron beam, reaching a decomposition of up to $30 \%$ with pure ammonia. They also analyzed the influence of the gases generated on the reaction rate, determining a favorable effect of the dilution of ammonia with $\mathrm{N}_{2}$ and an inhibition by $\mathrm{H}_{2}$. With the objective of generating hydrogen, McLennan and Greenwood $^{80}$ and Seabury et al. ${ }^{81}$ analyzed the decomposition of ammonia using electron beams on a $\mathrm{Ni}(111)$ catalyst. In a similar way, Hirabayashi and Ichihashi ${ }^{84}$ evaluated the decomposition reaction of ammonia through an ion beam using various vanadium and niobium nitrides, determining that some of them were promising for the generation of hydrogen. In another sense, and due to the corrosive characteristics of ammonia, Dawson and Peng, ${ }^{82}$ among others, sought to eliminate ammonia by exposing the corrosive gas to a tungsten surface in the presence of a low-energy electron beam, detecting ammonia conversion at temperatures as low as $27{ }^{\circ} \mathrm{C}$. At present, the decomposition of ammonia through an electron beam has also been proposed to eliminate its strong odor from industrial gas streams. ${ }^{83}$ For this, the decomposition in the presence of different types of substances such as helium, nitrogen, oxygen, or water has been evaluated, showing that the maximum efficiency of ammonia removal takes place in the presence of $\mathrm{O}_{2}$.

To provide heat to the reactor instead of using a conventional oven, the use of microwaves has been proposed, which allows reaching the desired temperature in a shorter time. In the work of Guler et al., ${ }^{85}$ mesoporous carbon was used as support for a catalyst composed of molybdenum. The ammonia conversion obtained in the microwave reactor was complete at $400{ }^{\circ} \mathrm{C}$, while using the same catalyst in a conventional oven under the same reaction conditions an ammonia conversion of only $49 \%$ at $600{ }^{\circ} \mathrm{C}$ was obtained. Similar results were obtained by Varışlı et $\mathrm{al}^{86}$ using an iron catalyst supported on mesoporous carbon, which was shown to completely convert ammonia in a microwave reactor at $450{ }^{\circ} \mathrm{C}$, while in a conventional reactor the complete conversion was reached at $600{ }^{\circ} \mathrm{C}$. In a previous work (1971) developed by Barker, ${ }^{87}$ the decomposition of ammonia through microwave discharges was examined, and the effect of adding scavengers of $\mathrm{H}$ atoms such as allyl alcohol and propylene promoted also the formation of hydrazine $\left(\mathrm{N}_{2} \mathrm{H}_{4}\right)$. 


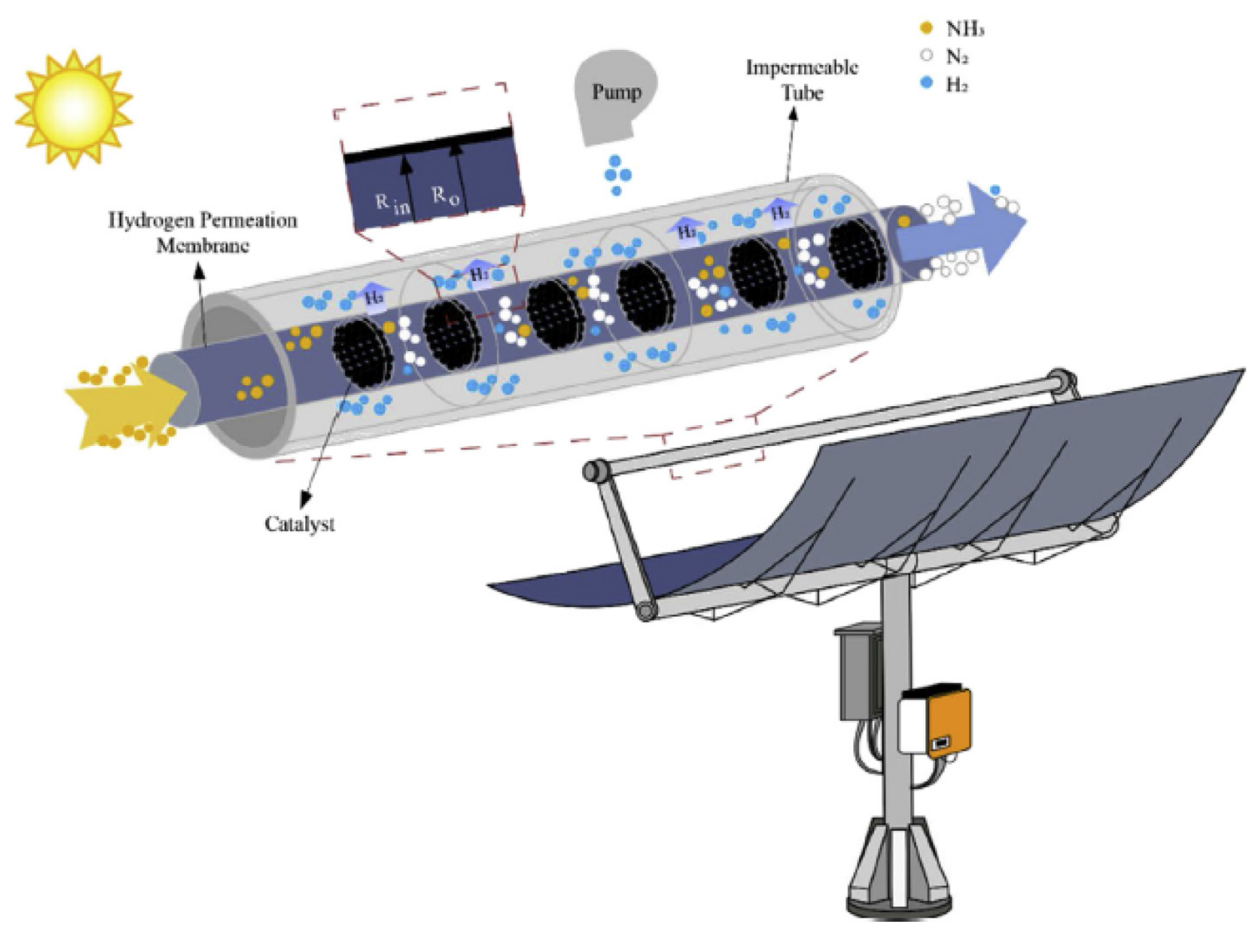

Figure 2. Conceptual diagram of the reactor equipment proposed by Wang et al. Reprinted with permission from ref 97 . Copyright 2019 Elsevier.

Another widely studied method to supply energy to the system is plasma technology. In the case of ammonia decomposition, the first work that proposed the use of plasma generated in an incandescent discharge tube of alternating voltage at low pressure was carried out by Carbaugh et al. ${ }^{88}$ in 1967 , also targeting the generation of hydrazine. Very low conversions were obtained, but no catalyst was used. More recently, the decomposition of ammonia through pulsed plasma or plasma generated by dielectric barrier discharges (DBD) in the presence of a catalyst $\left(\mathrm{Fe}^{89}\right.$ and $\left.\mathrm{Fe}-\mathrm{Ni}^{90}\right)$ or coupling plasma with a membrane reactor ${ }^{91}$ reached higher conversions. In a similar way, the work of Inoue et al. ${ }^{92}$ showed that complete conversion could be achieved at low temperatures with atmospheric pulsed plasma in the absence of catalysts by controlling the reaction conditions and dilution of ammonia with argon. However, the use of a stream of pure ammonia and DBD plasma resulted in lower conversions (19\%), according to Goto et al..$^{33}$

As shown by Akiyama et al. ${ }^{94}$ the type of electrodes used to produce the plasma does not interfere significantly in the decomposition of ammonia, whereas the applied power and the residence time of the gas shows a strong dependence on the conversion rate. Moreover, applying plasma technology at higher temperatures and in the presence of a low-cost catalyst results in a complete conversion at low temperatures. ${ }^{89,90}$ Different metals such as $\mathrm{Ni}, \mathrm{Co}, \mathrm{Fe}$, and $\mathrm{Mo}$ and their bimetallic forms $\mathrm{Fe}-\mathrm{Co}, \mathrm{Mo}-\mathrm{Co}, \mathrm{Fe}-\mathrm{Ni}$, and $\mathrm{Mo}-\mathrm{Ni}$ have been tested, and the catalyst composed of $\mathrm{Fe}-\mathrm{Ni}$ is the most active. ${ }^{90}$ Following the catalytic test performed by Yi et al., Wang et al. ${ }^{95}$ analyzed various elements and various supports as catalysts, among which $\mathrm{Co} / \mathrm{SiO}_{2}$ showed the strongest synergy with plasma, giving higher $\mathrm{NH}_{3}$ conversion. Finally, it is possible to use a combined system composed of a catalytic reactor and a plasma reactor with a hydrogen-selective membrane $(\mathrm{Pd}-\mathrm{Cu}$ alloy) to decrease the energy input necessary to decompose ammonia and obtain high energy efficiency. ${ }^{91}$ The results show a total hydrogen energy efficiency of about $30 \%$.

Within the framework of energy efficiency and to limit carbon emissions into the atmosphere, the production of hydrogen from ammonia using solar concentrators to convert renewable solar energy into chemical energy is currently being studied. In this sense, $\mathrm{Hu}$ et al. ${ }^{96}$ and Wang et al. ${ }^{97}$ are heading in this direction. At first, a catalyst composed of nickel supported on $\mathrm{Al}_{2} \mathrm{O}_{3}$ was used in a tubular reactor, in which the ammonia conversion was evaluated by changing its geometric characteristics and the reaction conditions, simulating through a model and obtaining an optimized reactor geometry, reaching conversions around $70 \%$ at a reactor wall temperature of $650{ }^{\circ} \mathrm{C}$. Subsequently, it was proposed to couple the solar concentrator with a catalytic membrane reactor $(\mathrm{Pd}-\mathrm{Ag}$ alloy), reaching almost complete conversion (99\%) at only $200^{\circ} \mathrm{C}$ at a pressure of $0.1 \mathrm{bar}$ (Figure 2 ). The low reaction temperatures lead to very high total energy efficiency.

Another method proposed in the literature to provide the necessary heat to the catalytic reactor is to couple the ammonia decomposition reaction with an exothermic reaction. This is the case proposed by Chen et al., ${ }^{98}$ who designed a microreactor where channels with a platinum catalyst were used for the combustion of a methane-air mixture. The gases are alternated with other channels for the decomposition of ammonia in the presence of ruthenium, obtaining a compact reactor of reduced size. Also Engelbrecht et al. ${ }^{99}$ and Chiuta et al. ${ }^{100}$ designed an autothermal microchannel reactor in which the decomposition of ammonia and its oxidation takes place in stainless steel plates alternated in parallel with $\mathrm{Ru}$ (decomposition) and $\mathrm{Pt}$ (oxidation) supported on alumina catalysts. Another application to couple ammonia decomposition with another exothermic reaction was proposed by Kim et al., ${ }^{101}$ where a microcombustor burns a mixture of $\mathrm{H}_{2}, \mathrm{NH}_{3}$, and air, and a microreformer converts $\mathrm{NH}_{3}$ to hydrogen using a ruthenium catalyst. The reformer is placed around the combustor to facilitate the transfer 
of heat. The ruthenium catalyst showed higher conversion rates compared to $\mathrm{Ni} / \mathrm{SiO}_{2}-\mathrm{Al}_{2} \mathrm{O}_{3}$ and Ir catalysts. ${ }^{102}$ Finally, Arana et al. ${ }^{103}$ coupled the combustion of butane with the decomposition of ammonia, and Kaisare et al. ${ }^{104,105}$ and Deshmukh and Vlachos ${ }^{106}$ used a similar microreactor using propane/air and ammonia.

The production of hydrogen in an aqueous medium from ammonia has also been considered, such as by alkaline electrolysis $^{107,108}$ or photocatalysis. ${ }^{109,110}$ Hanada et al. ${ }^{107}$ proposed the alkali metal amides $\mathrm{LiNH}_{2}, \mathrm{NaNH}_{2}$, and $\mathrm{KNH}_{2}$ as electrolytes. The results showed that $\mathrm{KNH}_{2}$ is the best compound among the three proposed for generating a high current density. The results showed that the electrolysis of ammonia is a valuable technology for the on-site generation of $\mathrm{H}_{2}$, also considering that it requires $94 \%$ less energy than the electrolysis of water. In the study by Modisha and Bessarabov, ${ }^{108}$ $\mathrm{KOH}$ was used as the electrolyte and platinum-iridium was used as the electrocatalyst. The influence of temperature and ammonia concentration on the conversion was studied, obtaining an output flow with an ammonia concentration less than $0.1 \mathrm{ppm}$, which allows its direct use in a PEMFC. In relation to the photocatalysis of ammonia in aqueous medium, different photocatalysts have been proposed, such as those based on titania, a widely studied photocatalyst, alone or doped with ceria, ${ }^{109}$ or $\mathrm{Ru} / \mathrm{ZnS} .{ }^{110}$ In the work by Reli et al. ${ }^{109}$ it was shown that doping titania with ceria increases ammonia conversion compared to undoped titania, whereas in the work of Iwase, $\mathrm{Li}$, and $\mathrm{Kudo}^{110} \mathrm{Ru} / \mathrm{ZnS}$ was active for the photocatalysis of ammonia under both ultraviolet and sunlight conditions. The photolysis of ammonia has also been tested in the gas phase. One of the first works on the photolysis of gaseous ammonia without a catalyst was carried out in 1932 by Wiig and Kistiakowsky. ${ }^{111}$ Later on, in $1983, \mathrm{Li}$ et al. ${ }^{112}$ tested several photocatalysts to convert ammonia, obtaining the best results with $\mathrm{Ru}-\mathrm{Ni}$ supported on $\mathrm{BaTiO}_{3}$. Importantly, they demonstrated that the photocatalytic decomposition of pure ammonia was almost zero, but in the presence of water vapor it increased to reach considerable $\mathrm{H}_{2}$ production values. More recently, Yuzawa et al. ${ }^{113}$ compared the photocatalytic results obtained in the presence of water vapor with those obtained with ammonia in an aqueous medium. The results showed that platinum supported on titania was the most active photocatalyst compared to $\mathrm{Cu}, \mathrm{Ni}$, $\mathrm{Au}, \mathrm{Rh}$, and $\mathrm{Pd}$ for gaseous ammonia photocatalysis in the presence of steam. Furthermore, comparing the conversion of ammonia in the presence and that in the absence of water vapor, it was concluded that the production of hydrogen in the first case was more than three times higher. In the case of aqueous photocatalysis, it was determined that the production of hydrogen increased considerably using hydrazine instead of ammonia. $\mathrm{SrTiO}_{3}$ and $\mathrm{BaTiO}_{3}$ prepared by mechanochemical methods have been tested at room temperature, and a conversion of 2.5 and $4.5 \%$ has been obtained, respectively. ${ }^{114}$

2.2. Decomposition in the Presence of Other Substances. The study of the decomposition of ammonia in the presence of other substances has had several objectives, among which the main ones are to increase the conversion of ammonia or to eliminate it from a gas flow. In the first case, the possibility of the generation of hydrogen from ammonia through other routes, such as the reaction between ammonia and metal hydrides as magnesium hydride $\mathrm{MgH}_{2}$, has also been investigated. ${ }^{115}$ The experiments in this case were carried out in a discontinuous reactor in the temperature range of 75-150 ${ }^{\circ} \mathrm{C}$ in the presence of catalysts, obtaining good results of $\mathrm{H}_{2}$ production using $\mathrm{Pd}-\mathrm{Cl}$ compounds. In another direction, White and Melville ${ }^{116}$ investigated in 1905 the decomposition of ammonia in the gas produced during the destructive distillation of coal. For this, the experiments focused on decomposition in the presence of combinations of various gases, $\mathrm{CO}, \mathrm{H}_{2} \mathrm{O}, \mathrm{H}_{2}$, and $\mathrm{N}_{2}$, determining that $\mathrm{H}_{2}$ and $\mathrm{N}_{2}$ could be considered inert, while $\mathrm{CO}$ and water vapor increased the decomposition reaction rate.

The presence of $\mathrm{NH}_{3}$ in a gas stream can also be undesirable, as for example in the gas produced by the gasification of coal, and high $\mathrm{NO}_{x}$ emissions can occur when the gas is burned in a gas turbine. In this sense, Chambers et al. ${ }^{117}$ studied the use of $\mathrm{CaO}$ to clean the hot gas stream produced in the gasification of coal by decomposing the $\mathrm{NH}_{3}$ present. The same phenomenon occurs in biomass gasification; Wang et al. ${ }^{118}$ studied nickel as catalyst for the decomposition reaction of ammonia for its removal in a wide range of temperatures (between 200 and $1000{ }^{\circ} \mathrm{C}$ ) at a pressure of 21 bar. The main difficulty of ammonia removal in the presence of gas from the gasification of hydrocarbons is the possible poisoning of the catalyst by carbon deposition. Nickel has shown a greater capacity to be regenerated, removing carbon through oxygen treatment, compared to catalysts composed of Ru. ${ }^{119}$ Other materials such as dolomite ${ }^{120}$ have also been tested, but dolomite deactivates in the presence of carbon and water vapor. In this regard, a long-term (6 years) lifetime experiment of Ni-based catalysts in the presence of carbon and/ or water compounds was proposed by Platonov and Stepanov, ${ }^{121}$ showing a constant deactivation. Pansare and Goodwin $^{122}$ determined that tungsten-based catalysts, in particular WZ, showed a constant long-term conversion in the presence of syngas $\left(\mathrm{CO}+\mathrm{H}_{2}\right)$ after an initial period of induction. Ohtsuka et al., ${ }^{123}$ studying a catalyst composed of $\mathrm{Fe}$ supported on carbon, determined that in the presence of syngas the catalytic activity decreased dramatically due to the deposition of carbon in the catalyst. However, the introduction of $\mathrm{CO}_{2}$ into the inlet gas stream showed a recovery of the conversion. In a later work, Ohtuska et al. ${ }^{128}$ studied the tolerance of $\mathrm{FeOOH}$ (limonite) to the presence of $\mathrm{H}_{2} \mathrm{~S}$, a product of gasification, obtaining that in the presence of ca. 500 ppm of $\mathrm{H}_{2} \mathrm{~S}$ the conversion remained constant, while at 2000 ppm the catalyst suffered a reversible deactivation. In an opposite way, Uemiya et al. ${ }^{129}$ showed that Fe-based catalysts can convert ammonia in the presence of $5000 \mathrm{ppm}$ of $\mathrm{H}_{2} \mathrm{~S}$ without being deactivated. Furthermore, when Ni was used as a catalyst, a deactivation of the catalyst was not detected in the presence of gas streams containing up to $150 \mathrm{ppm}$ of $\mathrm{H}_{2} \mathrm{~S}^{130}$ Additionally, the catalyst did not show deactivation by carbon deposition in the presence of tar. It is important to mention that the degree of sulfur poisoning during ammonia decomposition is related to the number of active sites of a catalyst. ${ }^{131}$ The removal of ammonia by decomposition from gas flows generated from coal gasification has also been coupled to membrane reactors, ${ }^{124-127}$ with which it was possible to achieve the complete destruction of ammonia in the presence of the lowcost catalysts $\mathrm{Ni} / \mathrm{Al}_{2} \mathrm{O}_{3}$ at relatively low temperatures (400 $\left.{ }^{\circ} \mathrm{C}\right) .{ }^{125}$

In the presence of oxygen, the decomposition of ammonia follows a different route, and depending on the amount of oxygen present in the reagent stream, it can decompose and generate $\mathrm{N}_{2}$ or nitrogen oxides, $\mathrm{NO}_{x}$. The oxidation of ammonia was studied for its elimination from gas streams when a selective catalyst toward the generation of $\mathrm{N}_{2}$ that prevents the formation of nitrogen oxides was sought. Theoretically, catalysts based on 


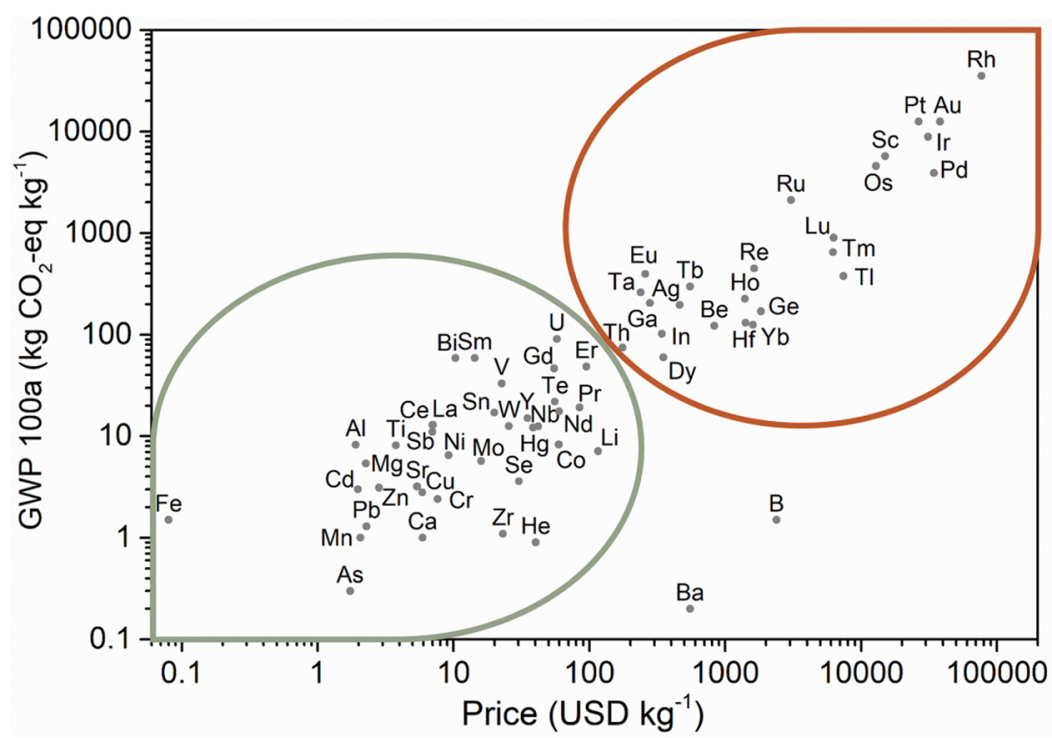

Figure 3. Comparison of the price $\left(\mathrm{USD} \mathrm{kg}^{-1}\right)^{146}$ and the global warming power on a 100 -year basis $\left(\mathrm{kg} \mathrm{CO}_{2}-\mathrm{equiv} \mathrm{kg}^{-1}\right)^{147}$ of the elements.

Ir can achieve $100 \%$ selectivity toward $\mathrm{N}_{2}{ }^{132}$ Hung $^{133,140}$ proposed a catalyst formed by $\mathrm{Cu}$ and ceria oxide, obtaining a selectivity toward the production of $\mathrm{N}_{2}$ up to $85 \%$ with an ammonia conversion of up to $98 \%$. Other studies on the selective oxidation of ammonia were carried out by Lee et al. ${ }^{134}$ using a bimetallic catalyst formed by $\mathrm{Ag}$ and $\mathrm{Cu}$ supported on alumina, and by Bera and Hedge, ${ }^{135}$ who tested different active phases supported on alumina or ceria, obtaining the best results with Pt. The oxidation of ammonia in the presence of oxygen has been also proposed for ammonia decomposition to generate $\mathrm{H}_{2}$ in the presence of a $\mathrm{RuO}_{2} / \mathrm{Al}_{2} \mathrm{O}_{3}$ catalyst. ${ }^{136}$ In this case, the asdeveloped process does not need an external source of heat to initiate or maintain the reaction due to the exothermicity of the ammonia oxidation reaction.

The decomposition of ammonia has also been studied as an intermediate reaction of the supercritical oxidation of water from organic waste. ${ }^{137}$ Supercritical oxidation is applied to destroy excess sludge from sewage treatment plants, and the reaction is performed with an oxidant such as hydrogen peroxide at temperatures between 450 and $550^{\circ} \mathrm{C}$. The decomposition of ammonia is one of the slowest stages in the supercritical oxidation process of sludge. The decomposition of ammonia into $\mathrm{N}_{2}$ and $\mathrm{H}_{2}$ in the presence of water vapor was studied using Ni-based catalysts supported on alumina; ${ }^{138,139}$ water vapor inhibited the conversion of ammonia and initially deactivated the catalyst until a constant conversion was reached.

\section{CATALYSTS FOR THE THERMAL DECOMPOSITION OF AMMONIA}

Thermal decomposition or catalytic cracking is the most common technique used for the generation of hydrogen from ammonia. It can be carried out with or without the presence of a catalyst, as the presence of a catalyst allows the decrease of the temperature necessary for the decomposition. For this reason, it is important to study the catalysts involved as well as different reactor configurations in order to decrease the supply of energy, in this case in the form of heat, to the system.

3.1. Catalysts. Since the decomposition of ammonia is the inverse reaction of the Haber-Bosh process for the synthesis of ammonia, initially the same catalysts used for the synthesis, $\mathrm{Ru}$ and $\mathrm{Fe},{ }^{141}$ were considered for the thermal decomposition of ammonia assuming the principle of microreversibility in heterogeneous catalysis. Afterward, $\mathrm{Cu}$-based catalysts were studied $^{142}$ as well as other metals, including Ni, Ir, Mo, Co, Pt, $\mathrm{Pd}$, and $\mathrm{Rh},{ }^{143}$ and different combinations of metals, such as $\mathrm{Co}-\mathrm{Mo}$, Ni-Mo, Fe-Mo, Ni-Co, $\mathrm{Co}-\mathrm{Mo}-\mathrm{Fe}-\mathrm{Ni}-\mathrm{Cu}$, $\mathrm{Mg}-\mathrm{Fe}, \mathrm{Fe}-\mathrm{Co}, \mathrm{Ni}-\mathrm{Fe}, \mathrm{Mg}-\mathrm{Co}-\mathrm{Fe}, \mathrm{Ni}-\mathrm{Pt}, \mathrm{Ni}-\mathrm{Pd}, \mathrm{Ir}-\mathrm{Ni}$, $\mathrm{Cu}-\mathrm{Zn}$, and bimetallic compositions with $\mathrm{Ru} .{ }^{144}$ Nowadays, the catalyst used commercially for the decomposition of ammonia is nickel supported on alumina, due to its mechanical properties and heat resistance. ${ }^{34}$ Among the catalysts studied in the literature, the highest catalytic activity for the decomposition of ammonia has been found with ruthenium supported on different oxides or structured and unstructured carbon, ${ }^{145}$ with the main problem being deactivation. ${ }^{44}$ However, ruthenium is a noble metal, rare in nature, and consequently an expensive element. For this reason, low-cost catalytic compositions with a catalytic activity comparable to that of ruthenium have been actively sought. ${ }^{143}$ In addition to the price of the metal on which the catalyst is based, within the framework of environmental sustainability it is recommended to also take into account the environmental impact of the material used, such as its global warming power or the energy demand of its extraction and refining. Figure 3 shows the relationship between the price of the different elements of the periodic table and their global warming power (GWP) over a period of 100 years. ${ }^{146,147}$ Generally, the price and the GWP have a linear relationship, since both are related to the abundance of an element on earth; the scarcer is the element, the higher is its price and the energy necessary for its availability. For this reason, noble metals, such as $\mathrm{Ru}$, are at the top of the chart, having a high price and high environmental impact. If possible, the use of the elements that are located in the lower part should be preferred, such as the transition metals $\mathrm{Fe}$, $\mathrm{Ni}$, Mo, or Co, among others.

The catalytic activity in the decomposition of ammonia depends not only on the active phase used, but also on the support and promoters. In general, the catalytic activity improves with supports that exhibit high surface area, with high dispersion and reduced particle size of the metal, and in the presence of electrodonating promoters such as $\mathrm{K}$, Cs, or Ba. ${ }^{148}$ The support can stabilize the size and/or morphology of the metal particles and increase the exposure of their active sites and, 


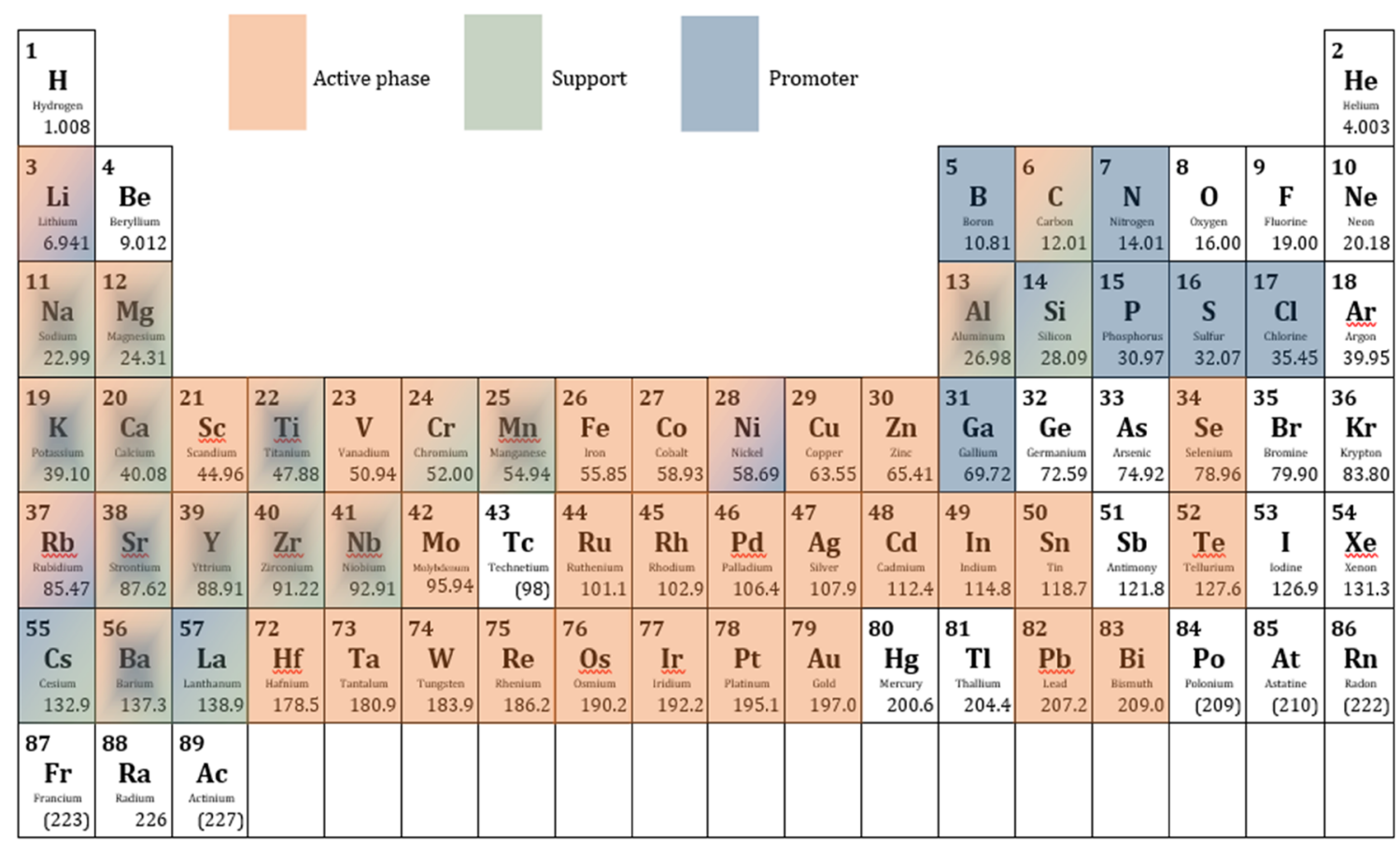

\begin{tabular}{|c|c|c|c|c|c|c|c|c|c|c|c|c|c|}
\hline $\begin{array}{l}58 \\
\mathrm{Ce}\end{array}$ & $\begin{array}{l}59 \\
\mathrm{Pr}\end{array}$ & 60 & $\begin{array}{c}61 \\
\text { Pm }\end{array}$ & ${ }^{62} \mathrm{Sm}$ & $\begin{array}{c}63 \\
\mathrm{Eu}\end{array}$ & 64 & 65 & $\begin{array}{l}66 \\
\text { Dy }\end{array}$ & $\begin{array}{c}67 \\
\text { Ho }\end{array}$ & $\begin{array}{l}68 \\
\mathrm{Er}\end{array}$ & 69 & $\begin{array}{l}70 \\
\mathrm{Yb}\end{array}$ & $\begin{array}{c}71 \\
\text { Lu }\end{array}$ \\
\hline 140.1 & 140.9 & 144.2 & (145) & 150.4 & 152.0 & 157.3 & 158.9 & 162.5 & 164.9 & 167.3 & 168.9 & 173.0 & 175.0 \\
\hline $\begin{array}{l}90 \\
\text { Th }\end{array}$ & $\begin{array}{l}91 \\
\mathrm{~Pa}\end{array}$ & $\begin{array}{r}92 \\
U\end{array}$ & & & & & & & & & & & \\
\hline 232.0 & 231.0 & 238.0 & & & & & & & & & & & \\
\hline
\end{tabular}

Figure 4. Simplified periodic table of the elements used in the formulation of catalysts for the decomposition of ammonia.

at the same time, it can affect the electronic structure of the supported metals. ${ }^{143}$ In particular, it has been determined that the basicity of the catalyst increases the catalytic activity ${ }^{149}$ as well as the ability to generate bonds with nitrogen atoms with a given energy. ${ }^{145}$ On the other hand, it must be considered that the composition of the reaction gas also influences the catalytic performance, being that the composition of the gas varies across the catalytic bed, which means that an optimal catalyst in the inlet conditions may not be optimal in the outlet, where there is a higher concentration of $\mathrm{H}_{2}$ and $\mathrm{N}_{2}$ and a lower concentration of $\mathrm{NH}_{3}{ }^{148}$ In Figure 4, the elements reported in the literature involved in the catalysts for the decomposition of ammonia are indicated, divided by active phase, support, and promoters. Some elements such as $\mathrm{Mg}, \mathrm{Al}$, or Ce are used for the three components, while others such as noble metals are used only as the active phase of the catalyst, or the alkali metals, that are used mainly as promoters.

The different catalysts reported in the literature for the decomposition of ammonia are presented according to their active phase in Tables 2-10 (section 3.1.1). The results reported correspond to those obtained at atmospheric pressure and in the presence of pure ammonia or diluted with an inert gas, assuming a pressure of $1 \mathrm{~atm}$ when not specified. The values of the conversion of ammonia, $\mathrm{H}_{2}$ formation rate, TOF, and apparent activation energy at the temperatures specified in the text are included in the tables. When not present in the text, the values were estimated by extrapolation, reporting representative values between 350 and $500{ }^{\circ} \mathrm{C}$. In the case that different methods were used for the synthesis of catalysts with the same composition, or that they had different relative amounts of the components, it was chosen to report only the results of the catalyst for which the higher catalytic activity was obtained. The reaction conditions are reported or calculated from the data provided in the different studies (if they are not indicated it is because they have not been specified by the authors). The works related to reaction kinetics studies are compiled in Table 11 (section 3.2), including results obtained at pressures other than atmospheric. Apparent activation energy values have been obtained from experiments as well as theoretical studies.

As far as review articles on the catalytic decomposition of ammonia are concerned, works that consider decomposition in the framework of hydrogen production as well as works that focus on the type of catalyst or structured support used have been found in the literature. The first are the works of Klerke et al. (2008), ${ }^{34}$ Zamfirescu and Dincer (2008-2009), ${ }^{35,48}$ Cheddie (2012), ${ }^{149}$ Lan et al. (2012), ${ }^{33}$ Giddey et al. (2017), ${ }^{36}$ Abd Ali et al. (2018), ${ }^{150}$ and Makepeace et al. (2019). ${ }^{141}$ Among those that analyze the catalysts, supports and types of reactor used are the works of Yin et al. (2004), ${ }^{151}$ Schüth et al. (2012), ${ }^{145}$ Chiuta et al. (2013), ${ }^{46}$ Duan et al. (2013), ${ }^{152}$ García-Bordejé et al. (2014), ${ }^{148}$ Bell and TorrenteMurciano (2016), ${ }^{144}$ Mukherjee et al. (2018), ${ }^{143}$ and Lamb et al. (2019). ${ }^{44}$

3.1.1. Importance of the Active Phase. There are several works focusing on a comparison of the catalytic activity of different metals supported on the same support for ammonia decomposition. Boisen et al. ${ }^{153}$ in 2005 carried out a decisive study; they compared different active phases (Fe, $\mathrm{Co}, \mathrm{Ni}, \mathrm{Cu}$ and 


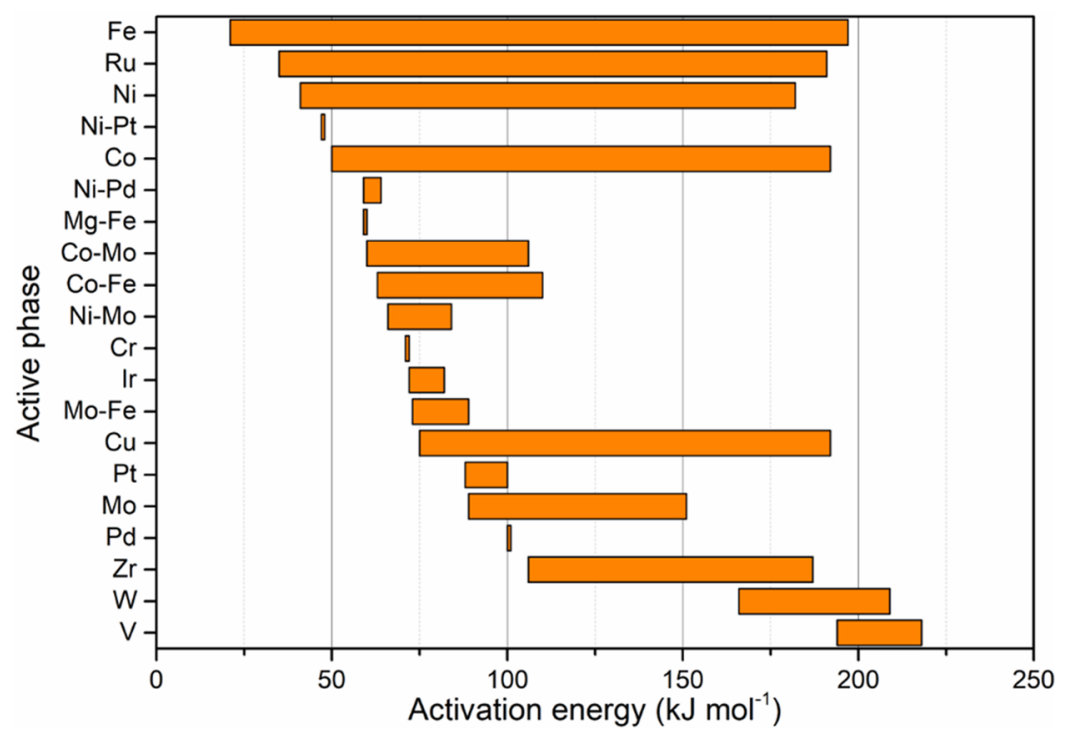

Figure 5. Apparent activation energy values for the decomposition of ammonia recorded on different metal-based catalysts.

$\mathrm{Ru}$ ) supported on $\mathrm{MgAl}_{2} \mathrm{O}_{4}$ spinel and observed that the best catalysts for the synthesis of ammonia are not good catalysts for its decomposition. They evaluated it by calculating the TOF values and comparing them with the dissociation energy of the nitrogen bond at the active sites, obtaining a volcano-shaped curve, with the optimum in the case of metals that can generate the less strong $\mathrm{N}$ bond. They also observed that the position of the maximum of the curve varied according to the reaction conditions, such as the concentration of ammonia. Through an interpolation in the periodic table, they proposed the catalyst composed of $\mathrm{Co}_{3} \mathrm{Mo}_{3} \mathrm{~N}$ as a good candidate for the decomposition of ammonia. ${ }^{154}$ This model, which focuses on the energy of nitrogen chemisorption, is the one that predicts more precisely the catalytic activity of metals. However, it cannot foresee the higher activity of rhodium compared to cobalt. ${ }^{155}$ Ganley et al. proposed that this occurs because the determining step of the decomposition reaction mechanism changes according to the catalyst. ${ }^{155}$ They compared the results of several monometallic catalysts supported on alumina and were able to define the following relative activity relationship for decomposition: $\mathrm{Ru}>\mathrm{Ni}>\mathrm{Rh}>\mathrm{Co}>\mathrm{Ir}>\mathrm{Fe} \gg \mathrm{Pt}>\mathrm{Cr}>\mathrm{Pd}>$ $\mathrm{Cu} \gg \mathrm{Te}, \mathrm{Se}, \mathrm{Pb}$. However, since the support has an important influence on the catalytic activity, the relationship between the catalytic activities of the catalysts can vary considerably depending on the support considered. For example, in the study by Yin et al. at a reaction temperature of $400{ }^{\circ} \mathrm{C}$, the TOF as well as the ammonia conversion of the catalysts supported on carbon nanotubes can be arranged in the order: $\mathrm{Ru}>\mathrm{Rh} \approx \mathrm{Ni}>$ $\mathrm{Pt} \approx \mathrm{Pd}>\mathrm{Fe},{ }^{156}$ different from the catalysts supported on alumina prepared by Ganley et al. ${ }^{155}$

Using a high-throughput method, Liu et al. ${ }^{157}$ tested a high number of transition metals supported on $\mathrm{SiO}_{2}$, verifying that the activity of the catalysts at $570^{\circ} \mathrm{C}$ is $\mathrm{Ru}>\mathrm{Ni}>\mathrm{Co}>\mathrm{Ir}>\mathrm{Ag}>$ $\mathrm{Mo}>\mathrm{Pd}>\mathrm{Gd}$, while the other metals showed a catalytic activity equal to or less than that obtained without a catalyst. They also determined that a nitriding pretreatment with $\mathrm{H}_{2} / \mathrm{N}_{2}$ can change the catalytic activity of the catalysts, as in the case of $\mathrm{Fe}$, Co, and Mo, which presented a much higher catalytic activity after pretreatment due to the formation of the respective nitrides. Also V, W, and Ti showed an increase in their activity after the pretreatment. Therefore, not only does the support but also the reaction conditions and the preparation method influence the activity of a catalyst. In this sense, Choudhary et al. ${ }^{158,159}$ using $\mathrm{SiO}_{2}$ as a support, determined the order of catalytic activity $\mathrm{Ru}>\mathrm{Ir}>\mathrm{Ni}$ at various temperatures using pure ammonia. By testing the $\mathrm{Ru}$ and $\mathrm{Ir}$ catalysts supported on $\mathrm{Al}_{2} \mathrm{O}_{3}$, they concluded that both metals present lower catalytic activity compared to when supported on $\mathrm{SiO}_{2},{ }^{158}$ but they follow the same relative trend. Comparing the active phases of $\mathrm{Fe}, \mathrm{Ni}$, and $\mathrm{Ru}$ with their encapsulated forms in $\mathrm{SiO}_{2}\left(@ \mathrm{SiO}_{2}\right)$ under the same reaction conditions, a significant increase in catalytic activity resulting from encapsulation was observed in all three cases (in the case of $\mathrm{Ru}$ the increase was the highest, ammonia conversion increased by $62 \%$ at a temperature of $500{ }^{\circ} \mathrm{C}$ ). ${ }^{160,161}$ The encapsulated catalysts showed the order of catalytic activity: $\mathrm{Ru}>\mathrm{Ni} \approx \mathrm{Fe}$. However, a Co catalyst prepared following the same technique presented lower activity than the other three formulations. ${ }^{162}$ On the contrary, when they were supported on carbon nanotubes, Co showed a higher catalytic activity with respect to Fe. ${ }^{163}$ Finally, promoters can also have a positive effect on one type of catalyst, while they decrease the activity of another type. $\mathrm{Li}$ et al. ${ }^{164}$ investigated different forms of $\mathrm{SiO}_{2}$ (fumed $\mathrm{SiO}_{2}, \mathrm{MCM}-41$, and SBA-15) as supports for $\mathrm{Ni}$ and $\mathrm{Ru}$ catalysts, showing that the catalysts supported on these materials are more active than those supported on conventional $\mathrm{SiO}_{2}$. In general, supported $\mathrm{Ru}$ catalysts are more active than supported $\mathrm{Ni}$ catalysts (a conversion about $20 \%$ higher under the same conditions and considering the same support), and MCM-41 is the best support for both metals. The modification of the $\mathrm{Ru}$ catalysts with $\mathrm{KOH}$ contributes to a significant increase of the catalytic activity, while in the case of $\mathrm{Ni}$ the promoter effect is minimal.

The apparent activation energy values for the different catalysts have been analyzed with respect to the active phase used, obtaining the graph presented in Figure 5. The apparent activation energy is very different depending on the metal used and the support. In general, it is possible to conclude that the lowest apparent activation energy values are found for iron, ruthenium, and nickel catalysts, which are also the most studied catalysts in the literature. It can be concluded that the catalytic activity has a strong dependence with the type of support and 
promoter. The lowest catalytic activity is obtained with the nitrides and carbides of $\mathrm{V}$ and $\mathrm{W}$.

3.1.2. Noble Metals. The noble metals are grouped by their resistance to oxidation and corrosion. There is no strict definition for them, and $\mathrm{Ru}, \mathrm{Rh}, \mathrm{Pd}, \mathrm{Ag}, \mathrm{Re}, \mathrm{Os}, \mathrm{Ir}, \mathrm{Pt}$, and $\mathrm{Au}$ are usually considered. ${ }^{165}$ Papapolymerou and Bontozoglou ${ }^{166}$ tested different noble metals in the form of wires or polycrystalline foils at low reaction pressures between 225 and $925{ }^{\circ} \mathrm{C}$ and determined that the reaction rate for the decomposition of ammonia followed the order $\mathrm{Ir}>\mathrm{Rh}>\mathrm{Pt}>$ $\mathrm{Pd}$. However, among the noble metals, ruthenium is the element most studied as a catalyst for the decomposition of ammonia.

3.1.2.1. Ruthenium. Hill and Torrente-Murciano ${ }^{167}$ reported the catalyst considered as the most active up to date in the decomposition of ammonia: $\mathrm{Ru} / \mathrm{CNTs}$ (ruthenium supported on carbon nanotubes) promoted with Cs (20 wt \%). In a followup study, they prepared the same catalyst with a graphitization treatment of the carbon nanotubes and a lower Cs content (4 wt $\%)$, obtaining a significant increase in the conversion compared to the catalyst with the support without graphitization under the same reaction conditions. ${ }^{168}$ Wang et al. ${ }^{169}$ tested other types of promoters for the $\mathrm{Ru} / \mathrm{CNTs}$ catalyst, obtaining the following order of promotion at $400{ }^{\circ} \mathrm{C}: \mathrm{K}>\mathrm{Na}>\mathrm{Li}>\mathrm{Ce}>\mathrm{Ba}>\mathrm{La}>\mathrm{Ca}$; all promoted catalysts showed a higher catalytic activity with respect to the unpromoted ones. In general, various types of carbon show very good results as supports of $\mathrm{Ru}$ catalysts, in their unpromoted or alkali metal-promoted form. For example, a very high TOF of about $35 \mathrm{~s}^{-1}$ was obtained at $400{ }^{\circ} \mathrm{C}$ with a $\mathrm{Ru}$ catalyst supported on carbon and promoted with $\mathrm{K}^{170}$ It is worth mentioning that nitrogen doping changes the properties of carbon and further increases the catalytic activity of the catalyst. Ru supported on ordered mesoporous carbon (OMC) doped with nitrogen presented a high catalytic activity, compared to other forms of carbon (CNTs, activated carbon or undoped OMC). ${ }^{171}$ Nitrogen-doping has also a positive effect on Ru catalysts supported on carbon nanotubes. ${ }^{172,173}$ Chen et al. ${ }^{174}$ showed that doping carbon nanotubes with nitrogen increases the metal dispersion. Using carbon nanofibers (CNFs) as a support for $\mathrm{Ru}$, Marco et al. ${ }^{175}$ showed that doping with $\mathrm{N}$ leads to an increase of the catalytic activity compared to the support without doping, and that if oxygen is introduced into the structure of the nanofibers instead of nitrogen, the activity does not increase. In general, carbon nanofibers are proven to be an optimal support for $\mathrm{Ru}$, presenting similar catalytic activity with respect to $\mathrm{Ru} / \mathrm{CNTs}$. Another important aspect to take into account is the $\mathrm{Ru}$ particle size. By varying the size of the $\mathrm{Ru}$ particles the ammonia conversion has been optimized, obtaining the best results with a Ru particle size of about $2 \mathrm{~nm} .{ }^{176}$ On the contrary, activated carbon (AC) did not show to have superior activity with respect to carbon nanotubes, even if promoting a $\mathrm{Ru}$ catalyst supported on activated carbon with $\mathrm{Na} .{ }^{177,178}$ Yin et al. tested the catalysts $\mathrm{Ru} / \mathrm{MgO}$ and $\mathrm{Ru} / \mathrm{AC}$ promoted with $\mathrm{K}$, obtaining an increase in the conversion in both cases, particularly with the catalyst supported on activated carbon. ${ }^{179}$

Yin et al. ${ }^{156}$ carried out an analysis of the catalytic activity of $\mathrm{Ru}$ supported on various oxides and forms of carbon and obtained the order: CNTs $>\mathrm{MgO}>\mathrm{TiO}_{2}>\mathrm{Al}_{2} \mathrm{O}_{3}>\mathrm{ZrO}_{2}>\mathrm{AC}$. They attributed the superior catalytic activity of the CNTs to a greater dispersion of $\mathrm{Ru}$ and to its high electronic conductivity, which allows a greater transfer of electrons and, consequently, facilitates the desorption of $\mathrm{N}_{2}$ from the catalyst. They also tested the same catalysts prepared with acetone as solvent, instead of water, obtaining an increase in the ammonia conversion, which highlights the importance of the catalyst preparation method. ${ }^{180}$ Petrunin et al. analyzed the best ratio between $\mathrm{K}$ and $\mathrm{Ru}$ on mesoporous carbon (Sibunit) and obtained an increase in ammonia conversion of around $10 \%$ with respect to the catalyst without promoter at $450{ }^{\circ} \mathrm{C}$ with a $\mathrm{K} / \mathrm{Ru}$ molar ratio of 0.5 . Nevertheless, further increase of the amount of $\mathrm{K}$ produced a strong decrease in conversion. ${ }^{181}$ By changing the carbonaceous support, Li et al. ${ }^{182}$ tested a catalyst composed of $\mathrm{Ru}$ supported on graphene, which resulted in a high conversion of ammonia ( $91 \%$ at $450{ }^{\circ} \mathrm{C}$ ) using pure ammonia. In another work, $\mathrm{Li}$ et al. also tested other types of carbon as support for $\mathrm{Ru}{ }^{183}$ obtaining that the catalytic activity of the supports follows the order: graphitic carbon $>\mathrm{CNTs}>$ black carbon $>$ mesoporous carbon CMK-3 $>$ activated carbon. They also tested $\mathrm{Cr}_{2} \mathrm{O}_{3}$ as a support for a $\mathrm{Ru}$ catalyst ${ }^{184}$ and analyzed the relationship between $\mathrm{Ru}$ particle size and conversion, obtaining the maximum of activity with $4 \mathrm{~nm}$ particles. The catalytic activity of chromium oxide without ruthenium was also studied, and it was observed that the catalytic activity increased by increasing the size of the $\mathrm{Cr}_{2} \mathrm{O}_{3}$ particles. ${ }^{184}$ Raróg-Pilecka et al. ${ }^{185}$ tested a catalyst composed of $\mathrm{Ru}$ supported on carbon modified with $\mathrm{Cs}$ and $\mathrm{Ba}$, determining that for the whole range of concentrations of ammonia tested $(5-50 \%)$, Cs has a promoting effect greater than $\mathrm{Ba}$. Also Huang et al. ${ }^{186}$ obtained that $\mathrm{Cs}$ is an optimal promoter for the $\mathrm{Ru} / \mathrm{C}$ catalyst, obtaining an increase in conversion of $48 \%$ at $400{ }^{\circ} \mathrm{C}$ with respect to the unmodified catalyst using pure ammonia. Not only have alkali metals showed a promoting effect on carbon supported ruthenium catalysts, but also other elements. For example, Li et al. ${ }^{187}$ tested a catalyst composed of Ru supported on ordered mesoporous carbon (CMK-3) and evaluated different materials as promoters for the catalyst, obtaining a relative activity of $\mathrm{K}>$ $\mathrm{Na}>\mathrm{Ca}>\mathrm{Cl}>\mathrm{SO}_{4}>\mathrm{PO}_{4}>\mathrm{Li}$. The Li-modified catalyst showed lower activity with respect to the unpromoted catalyst.

Various forms of carbon mixed with other compounds, mainly oxides, were also tested as Ru supports. For example, Yin et al. ${ }^{188}$ compared the activity of Ru catalysts supported on CNTs, $\mathrm{MgO}$ and a mixture of the two, and they verified a synergy between the supports, especially when the catalyst is promoted with $\mathrm{K}$. Under the same conditions, the conversion of ammonia at $400{ }^{\circ} \mathrm{C}$ reached $80 \%$ while the nonpromoted $\mathrm{Ru} / \mathrm{CNTs}-\mathrm{MgO}$ catalyst reached a conversion of only $13 \%$. In a similar manner, Bajus et al. ${ }^{189}$ verified whether the same alkali metals that showed a promoting effect on the catalytic activity of $\mathrm{Ru}$ supported on carbon could be used to promote $\mathrm{Ru} / \mathrm{Al}_{2} \mathrm{O}_{3}$. They reported that the three metals $\mathrm{Li}, \mathrm{Na}$, and $\mathrm{Cs}$ do indeed have the ability to promote the catalyst. With $\mathrm{Na}$ and Cs, TOF was approximately twice that of the unpromoted catalyst, while $\mathrm{Li}$ presented the highest increase $\left(0.26\right.$ vs $0.05 \mathrm{~s}^{-1}$ at $\left.350^{\circ} \mathrm{C}\right)$. Moreover, Pyrz et al. ${ }^{190}$ identified $\mathrm{Ba}, \mathrm{Cs}$, and $\mathrm{K}$ as more promising with respect to $\mathrm{Sr}, \mathrm{Rb}, \mathrm{Ca}, \mathrm{Na}$, and $\mathrm{Li}$ when used as promoters of ruthenium supported on alumina. They also prepared catalysts using a combination of promoters, such as $\mathrm{Ru} / \mathrm{Al}_{2} \mathrm{O}_{3}$ promoted with $\mathrm{Ba}$ and $\mathrm{K}$ or $\mathrm{Cs}$ and $\mathrm{K}$. They found that the use of double promoted catalysts did not increase catalytic activity.

Moving on to a different type of supports, Klerke et al. ${ }^{191}$ compared $\mathrm{TiO}_{2}$ with three types of titanates $\left(\mathrm{Na}_{2} \mathrm{Ti}_{3} \mathrm{O}_{7}\right.$, $\mathrm{K}_{2} \mathrm{Ti}_{6} \mathrm{O}_{13}$ and $\mathrm{Cs}_{2} \mathrm{Ti}_{6} \mathrm{O}_{13}$ ) as support for $\mathrm{Ru}$, obtaining the same general trend to promote ruthenium catalysts with alkali metals: $\mathrm{Cs}>\mathrm{K}>\mathrm{Na}$. Comparing $\mathrm{Ru}$ supported on $\mathrm{Al}_{2} \mathrm{O}_{3}$ and Ladoped $\mathrm{Al}_{2} \mathrm{O}_{3}$, Chung et al. ${ }^{192}$ obtained a higher catalytic activity in the second case; at a temperature of $600{ }^{\circ} \mathrm{C}$ the conversion increased from 4 to $98 \%$ by adding $50 \mathrm{~mol} \% \mathrm{La}$ to the support, 
and the catalyst showed high stability in a $120 \mathrm{~h}$ test. Lanthanum has been used not only as a promoter, but also as a support; Huang et al. ${ }^{193}$ determined that the activity of $\mathrm{Ru}$ catalysts supported on $\mathrm{La}_{2} \mathrm{O}_{3}$ is higher than $\mathrm{Ru}$ supported on $\mathrm{Al}_{2} \mathrm{O}_{3}$, $\mathrm{Er}_{2} \mathrm{O}_{3}, \mathrm{SiO}_{2}$, and $\mathrm{TiO}_{2}$, and that the addition of $\mathrm{KOH}$ can effectively improve the catalytic activity of the catalysts. Szmigiel et al. $^{194}$ tested a $\mathrm{Ru}$ catalyst deposited on a magnesiumaluminum spinel and compared its activity with the same catalyst doped with $\mathrm{Ba}$ and $\mathrm{Cs}$. Also in the case of $\mathrm{MgAl}_{2} \mathrm{O}_{4}$ the two alkali metals are good promoters and the activity increased about 10 times compared to the unpromoted catalyst, although it is still lower than that obtained with the $\mathrm{Ru} / \mathrm{C}$ catalyst promoted with $\mathrm{Cs}$ and Ba. A similar composition was tested by Su et al., ${ }^{195}$ who prepared a $\mathrm{Ru}$ catalyst supported on a double layer oxide composed of $\mathrm{Mg}$ and $\mathrm{Al}$, obtaining a higher activity with respect to the $\mathrm{Ru}$ supported on the corresponding $\mathrm{MgO}$ and $\mathrm{Al}_{2} \mathrm{O}_{3}$ oxides separately. A Ru catalyst supported on $\mathrm{MgO}$ and $\mathrm{Al}_{2} \mathrm{O}_{3}$ was also tested by Tan et al., ${ }^{196}$ obtaining an increase of ammonia conversion of $44 \%$ at $500{ }^{\circ} \mathrm{C}$ with respect to the catalyst supported only on alumina.

Also zirconium oxide has proven to be a good support for ruthenium. Using a $\mathrm{Ru} / \mathrm{ZrO}_{2}$ catalyst, Wang et al. ${ }^{197}$ tested different zirconia supports modified with $\mathrm{Ba}$. As a promoter of the active phase, it presented a lower conversion of ammonia compared to the unpromoted catalyst, whereas when it was used as a promoter of the zirconia support a spectacular increase in activity was achieved. The conversion was further increased by adding $\mathrm{K}$ and $\mathrm{Cs}$ as promoters, obtaining the best results with Cs. By incorporating $\mathrm{Ru}$ nanoparticles into a La-doped $\mathrm{ZrO}_{2}$ support, Lorenzut et al. ${ }^{198}$ obtained a significantly higher activity with respect to the reference catalysts prepared by impregnation using $\mathrm{ZrO}_{2}-\mathrm{La}_{2} \mathrm{O}_{3}$ as support ( 81 vs $4 \%$ at $450{ }^{\circ} \mathrm{C}$ ). In a similar way, Miyamoto et al. ${ }^{199}$ modified a $\mathrm{Ru} / \mathrm{ZrO}_{2}$ catalyst with different amounts of $\mathrm{La}, \mathrm{Ca}$, and $\mathrm{Sr}$ obtaining a higher catalytic activity with respect to the unmodified catalyst in all three cases. Also, the subsequent promotion of $\mathrm{Ru}-\mathrm{La} / \mathrm{ZrO}_{2}$ with $\mathrm{Mg}$, Ca, and $\mathrm{Sr}$ did not allow an increase in the conversion of ammonia; nevertheless, the catalyst promoted with La and Sr presented the highest TOF value. Differently, Yin et al. ${ }^{200}$ modified a $\mathrm{Ru}$ catalyst supported on $\mathrm{ZrO}_{2}$ with $\mathrm{KOH}$ to increase the basicity of the catalyst, obtaining a better ammonia conversion than the catalyst further promoted with $\mathrm{K}\left(\mathrm{KOH}-\mathrm{Ru} / \mathrm{KOH}-\mathrm{ZrO}_{2}\right)$. On the contrary, Zhang et al. ${ }^{201}$ modified a Ru catalyst supported on $\mathrm{MgO}$ with $\mathrm{K}$ and $\mathrm{Cs}$ and obtained a higher ammonia conversion. The promoter effect using $\mathrm{K}$ with a catalyst composed of $\mathrm{Ru}$ supported on $\mathrm{MgO}$ was confirmed by Ju et al. ${ }^{202}$

Other less conventional supports have been proposed for $\mathrm{Ru}$ catalysts, for example an organic-metal chromium terephthalate structure (MIL-101) mixed with $\mathrm{MgO}^{203}$ The catalyst presented a similar catalytic activity with respect to when the support is not mixed with magnesia. However, after doping the two catalysts with Cs, the activity showed a significant increase, and the catalyst supported on MgO-MIL-101 presented the greatest increase. To lower the cost of the catalyst made up of $\mathrm{Ru}$, red mud was tested as support. ${ }^{204} \mathrm{Red}$ mud is composed mainly of the oxides of $\mathrm{Al}, \mathrm{Si}, \mathrm{Ca}$, and $\mathrm{Fe}$ and it is a residual material from the alumina production process. High ammonia conversion was obtained, comparable with other configurations of Ru catalysts, and stable over $170 \mathrm{~h}$. Another waste material that has been tested as $\mathrm{Ru}$ support for the ammonia decomposition reaction is fly ash, composed mainly of $\mathrm{SiO}_{2}$ and $\mathrm{Al}_{2} \mathrm{O}_{3}$ with the presence of $\mathrm{Fe}$, but the results are not as good as with red mud. 205,206
Silica is another support that has been extensively tested as a catalytic support, alone or mixed with other materials, and also as a natural mineral. A catalyst composed of $\mathrm{Ru}$ on $\mathrm{SiO}_{2}$ showed high catalytic activity, reaching a conversion of $86 \%$ at $500{ }^{\circ} \mathrm{C}$ with a high space velocity of pure ammonia. The catalytic activity is also promoted by $\mathrm{K}$, and the ammonia conversion increases by $50 \%$ at $600{ }^{\circ} \mathrm{C}$ with respect to nonpromoted catalysts. $^{207,208}$ A natural material, halloysite nanotubes (HNTs), composed mainly of $\mathrm{SiO}_{2}, \mathrm{Al}_{2} \mathrm{O}_{3}$, and other impurities, was used as a support for $\mathrm{Ru}$ and tested in the decomposition of ammonia. ${ }^{209}$ Although the catalytic performance of $\mathrm{Ru} / \mathrm{HNTs}$ is not comparable to that of $\mathrm{Ru} / \mathrm{CNTs}$, the former has the advantages of using a low cost and highly abundant support.

Cerium oxide is a material that allows easy control of the shape of its particles by varying the synthesis conditions. A low amount of $\mathrm{Ru}$ ( 1 wt \%) supported on two types of $\mathrm{CeO}_{2}$ nanoshapes, nanorods, and nanospheres, showed high catalytic activity ( 32 and $25 \%$ at $350{ }^{\circ} \mathrm{C}$, respectively) compared to the catalyst supported on $\mathrm{Al}_{2} \mathrm{O}_{3}$ and $\mathrm{MgO}$, with a conversion of only 5 and $11 \%$ under the same reaction conditions. ${ }^{210}$ Comparing different $1 \mathrm{D}$ nanoshapes of ceria, alumina, titanate, and carbon, $\mathrm{Hu}$ et al. ${ }^{211}$ found that $\mathrm{CeO}_{2}$ nanorods were the best support for $\mathrm{Ru}$ catalyst compared to CNTs and $\mathrm{Al}_{2} \mathrm{O}_{3}$, particularly at low temperatures. This result is attributed to the strong metalsupport interaction and the electronic modification of $\mathrm{Ru}$ by ceria. The addition of $\mathrm{Na}$ to the catalyst improved the activity when CNTs and $\mathrm{Al}_{2} \mathrm{O}_{3}$ were used as supports, but had a negligible effect on $\mathrm{CeO}_{2}$-based catalysts.

The basicity of Ru-based catalyst was proven to be important. Comparing catalysts based on $\mathrm{Ru}$ promoted with $\mathrm{K}$ supported on different materials (CNTs, $\mathrm{MgO}$, sepiolite, and $\mathrm{CaO}$ ), Sayas et al. ${ }^{73}$ obtained the highest catalytic activity with $\mathrm{CaO}$, which is the most basic support of those used. The $\mathrm{Ru}$ supported on a layered oxide composed of $\mathrm{Ca}$ and $\mathrm{Al}\left(\mathrm{CaAlO}_{x}\right)$ was compared with other types of supports widely used in the literature, carbon nanotubes and $\mathrm{SiO}_{2}$, obtaining a higher conversion (78 vs 21 and $62 \%$ at $450{ }^{\circ} \mathrm{C}$, respectively). ${ }^{212}$ In a similar way, Hayashi et $\mathrm{al}^{213}$ tested as supports an electride, $\left[\mathrm{Ca}_{24} \mathrm{Al}_{28} \mathrm{O}_{64}\right]^{4+}\left(\mathrm{e}^{-}\right)_{4}$, where electrons work as anions, and mayenite oxide $\left[\mathrm{Ca}_{24} \mathrm{Al}_{28} \mathrm{O}_{64}\right]^{4+}\left(\mathrm{O}^{2-}\right)_{2}$, and compared the results with conventional catalysts of $\mathrm{Ru}$ supported on $\mathrm{CaO}, \mathrm{Al}_{2} \mathrm{O}_{3}, \mathrm{MgO}$. They also prepared a K-promoted $\mathrm{Ru} / \mathrm{MgO}$ and Cs-promoted $\mathrm{Ru} / \mathrm{C}$ catalyst. At relatively low temperatures $\left(400{ }^{\circ} \mathrm{C}\right)$ the catalyst supported on $\left[\mathrm{Ca}_{24} \mathrm{Al}_{28} \mathrm{O}_{64}\right]^{4+}\left(\mathrm{e}^{-}\right)_{4}$ showed the highest conversion. Barium has also shown to be a catalyst promoter when used to modify an alumina support. For instance, $\mathrm{Ru}$ supported on barium hexaaluminate (BHA) shows superior catalytic activity compared to traditional supports such as $\mathrm{MgO}$, $\mathrm{Al}_{2} \mathrm{O}_{3}$, and CNTs; around 4 times higher compared to $\mathrm{MgO}$ and $\mathrm{Al}_{2} \mathrm{O}_{3}$ supports, and more than double compared to carbon nanotubes. A very good stability for $60 \mathrm{~h}$ was reported using pure ammonia at $450{ }^{\circ} \mathrm{C} .^{214}$

An intensive analysis of the catalytic activities of $\mathrm{Ru}$ supported on $\operatorname{Pr}_{6} \mathrm{O}_{11}$ was carried out by Nagaoka et al. ${ }^{215}$ The ammonia conversion on promoted $\operatorname{Pr}_{6} \mathrm{O}_{11}$ followed the trend $\mathrm{Cs}_{2} \mathrm{O}>$ $\mathrm{Rb}_{2} \mathrm{O}>\mathrm{K}_{2} \mathrm{O}>\mathrm{Na}_{2} \mathrm{O}$, where $\mathrm{Cs}_{2} \mathrm{O}$ is the most basic oxide. $\mathrm{Ru} /$ $\mathrm{Pr}_{6} \mathrm{O}_{11}$ promoted with $\mathrm{Cs}_{2} \mathrm{O}$ showed a very high ammonia conversion. Comparing the nonpromoted $\mathrm{Ru} / \operatorname{Pr}_{6} \mathrm{O}_{11}$ catalyst with $\mathrm{Ru}$ supported on other oxides, they obtained a similar catalytic activity with $\mathrm{Ru} / \mathrm{La}_{2} \mathrm{O}_{3}$. Moreover, when $\mathrm{Ru} / \mathrm{La}_{2} \mathrm{O}_{3}$ is promoted with $\mathrm{Cs}$, the activity increases, but to a lesser extent with respect to the promoted $\mathrm{Ru} / \mathrm{Pr}_{6} \mathrm{O}_{11}$ catalyst. $^{216} \mathrm{Ru}$ supported on $\mathrm{MgO}, \mathrm{Al}_{2} \mathrm{O}_{3}$, and $\mathrm{SiO}_{2} / \mathrm{Al}_{2} \mathrm{O}_{3}$ showed a low 
Table 2. Catalysts Based on Ruthenium Used to Decompose Ammonia and Their Catalytic Performances at 1 atm

\begin{tabular}{|c|c|c|c|c|c|c|c|c|c|c|c|c|}
\hline $\begin{array}{l}\text { active } \\
\text { phase }\end{array}$ & wt $\%$ & support & promoter & $\begin{array}{c}\text { WHSV } \\
\left(\mathrm{mL} \mathrm{g}^{-1} \mathrm{~h}^{-1}\right)\end{array}$ & $\begin{array}{c}\text { GHSV } \\
\left(\mathrm{h}^{-1}\right)\end{array}$ & $\begin{array}{c}\% \mathrm{NH}_{3} \\
\text { inlet flow }\end{array}$ & $\begin{array}{l}T\left(^{\circ}\right. \\
\mathrm{C})\end{array}$ & $\begin{array}{l}\text { conv } \\
(\%)\end{array}$ & $\begin{array}{c}\mathrm{H}_{2} \text { rate } \\
\left(\mathrm{mmol} \mathrm{g}^{-1} \mathrm{~s}^{-1}\right)\end{array}$ & $\begin{array}{c}E_{\mathrm{a}} \\
\left(\mathrm{kJ} \mathrm{\textrm {mol } ^ { - 1 }}\right)\end{array}$ & $\begin{array}{l}\text { TOF } \\
\left(s^{-1}\right)\end{array}$ & ref \\
\hline $\mathrm{Ru}$ & & & & 30000 & & 100 & 500 & 34 & 0.34 & & & 161 \\
\hline $\mathrm{Ru}$ & 3 & $@ \mathrm{La}_{2} \mathrm{O}_{3}-\mathrm{ZrO}_{2}$ & & 4000 & & 100 & 450 & 81 & & & & 198 \\
\hline $\mathrm{Ru}$ & 3 & $@ \mathrm{La}_{2} \mathrm{O}_{3}-\mathrm{ZrO}_{2}$ & $\mathrm{NaBH}_{4}$ & 4000 & & 100 & 450 & 63 & & & & 198 \\
\hline $\mathrm{Ru}$ & & $@ \mathrm{SiO}_{2}$ & & 30000 & & 100 & 500 & 96 & 0.50 & 41 & 18.1 & 161 \\
\hline $\mathrm{Ru}$ & & $@ \mathrm{SiO}_{2}$ & & 360000 & & 100 & 600 & 23 & & & & 207 \\
\hline $\mathrm{Ru}$ & & $@ \mathrm{SiO}_{2}$ & & 30000 & & 100 & 500 & 84 & & & & 208 \\
\hline $\mathrm{Ru}$ & & $@ \mathrm{SiO}_{2}$ & $\mathrm{~K}$ & 360000 & & 100 & 600 & 73 & & & & 207 \\
\hline $\mathrm{Ru}$ & 2.0 & {$\left[\mathrm{Ca}_{24} \mathrm{Al}_{28} \mathrm{O}_{64}\right]^{4+}\left(\mathrm{O}^{2-}\right)_{2}$} & & 15000 & & 30 & 400 & 42 & & 69 & 1.0 & 213 \\
\hline $\mathrm{Ru}$ & 2.2 & {$\left[\mathrm{Ca}_{24} \mathrm{Al}_{28} \mathrm{O}_{64}\right]^{4+}\left(\mathrm{e}^{-}\right)_{4}$} & & 15000 & & 30 & 400 & 70 & & 55 & 6.9 & 213 \\
\hline $\mathrm{Ru}$ & 2 & $\mathrm{AC}$ & & 20000 & & 10 & 350 & 25 & & & 0.07 & 178 \\
\hline $\mathrm{Ru}$ & 4.8 & $\mathrm{AC}$ & & 30000 & & 100 & 450 & & 0.16 & 75 & 1.7 & 180 \\
\hline $\mathrm{Ru}$ & 5.0 & $\mathrm{AC}$ & & 6000 & & 100 & 450 & 9 & & & & 171 \\
\hline $\mathrm{Ru}$ & 5 & $\mathrm{AC}$ & & 150000 & & 100 & 500 & 9 & 0.29 & 76 & & 156 \\
\hline $\mathrm{Ru}$ & 5 & $\mathrm{AC}$ & & 30000 & & 100 & 550 & 14 & 0.07 & & & 183 \\
\hline $\mathrm{Ru}$ & 5 & $\mathrm{AC}$ & & 15000 & & 100 & 400 & 4 & & & & 203 \\
\hline $\mathrm{Ru}$ & 5 & $\mathrm{AC}$ & $\mathrm{K}$ & 30000 & & 100 & 450 & 40 & & & & 179 \\
\hline $\mathrm{Ru}$ & 2 & $\mathrm{AC}$ & $\mathrm{Na}$ & 20000 & & 10 & 350 & 46 & & & 0.14 & 178 \\
\hline $\mathrm{Ru}$ & 0.5 & $\mathrm{Al}_{2} \mathrm{O}_{3}$ & & & & 100 & 580 & & & & 6.85 & 155 \\
\hline $\mathrm{Ru}$ & 0.7 & $\mathrm{Al}_{2} \mathrm{O}_{3}$ & & 2300 & & 10 & 500 & 4 & & & & 192 \\
\hline $\mathrm{Ru}$ & 1 & $\mathrm{Al}_{2} \mathrm{O}_{3}$ & & 22000 & & 100 & 350 & 5 & & & 0.2 & 210 \\
\hline $\mathrm{Ru}$ & 1 & $\mathrm{Al}_{2} \mathrm{O}_{3}$ & & 3000 & & 100 & 350 & 7 & & & & 216 \\
\hline $\mathrm{Ru}$ & 1.9 & $\mathrm{Al}_{2} \mathrm{O}_{3}$ & & 30000 & & 100 & 500 & 85 & & 80 & & 217 \\
\hline $\mathrm{Ru}$ & 2 & $\mathrm{Al}_{2} \mathrm{O}_{3}$ & & 30000 & & 100 & 500 & 42 & & & & 196 \\
\hline $\mathrm{Ru}$ & 2.0 & $\mathrm{Al}_{2} \mathrm{O}_{3}$ & & 15000 & & 30 & 400 & 13 & & 86 & 1.0 & 213 \\
\hline $\mathrm{Ru}$ & 2 & $\mathrm{Al}_{2} \mathrm{O}_{3}$ & & 240000 & 80000 & 50 & 500 & 98 & & & & 218 \\
\hline $\mathrm{Ru}$ & 2 & $\mathrm{Al}_{2} \mathrm{O}_{3}$ & & 13800 & 3067 & 57 & 400 & 37 & & & 1.0 & 219 \\
\hline $\mathrm{Ru}$ & 2.1 & $\mathrm{Al}_{2} \mathrm{O}_{3}$ & & 30000 & & 100 & 450 & 7 & & 122 & 0.6 & 214 \\
\hline $\mathrm{Ru}$ & 4 & $\mathrm{Al}_{2} \mathrm{O}_{3}$ & & & & 10 & 400 & 28 & & & & 190 \\
\hline $\mathrm{Ru}$ & 4 & $\mathrm{Al}_{2} \mathrm{O}_{3}$ & & 125000 & & 100 & 450 & 33 & & 95 & & 220 \\
\hline $\mathrm{Ru}$ & 4 & $\mathrm{Al}_{2} \mathrm{O}_{3}$ & & 120000 & & 10 & 350 & 24 & & 130 & 1.5 & 221 \\
\hline $\mathrm{Ru}$ & 4 & $\mathrm{Al}_{2} \mathrm{O}_{3}$ & & 5400 & & 100 & 400 & & & 125 & 0.14 & 222 \\
\hline $\mathrm{Ru}$ & 4.7 & $\mathrm{Al}_{2} \mathrm{O}_{3}$ & & 4160 & & 67 & 350 & & & & 0.05 & 189 \\
\hline $\mathrm{Ru}$ & 4.8 & $\mathrm{Al}_{2} \mathrm{O}_{3}$ & & 30000 & & 100 & 450 & & 0.13 & 61 & 2.5 & 180 \\
\hline $\mathrm{Ru}$ & 4.8 & $\mathrm{Al}_{2} \mathrm{O}_{3}$ & & 18000 & & 100 & 350 & 13 & & & & 193 \\
\hline $\mathrm{Ru}$ & 5 & $\mathrm{Al}_{2} \mathrm{O}_{3}$ & & 150000 & & 100 & 500 & 11 & 0.30 & 65 & & 156 \\
\hline $\mathrm{Ru}$ & 5 & $\mathrm{Al}_{2} \mathrm{O}_{3}$ & & 30000 & & 100 & 550 & 78 & & & & 223 \\
\hline $\mathrm{Ru}$ & 5 & $\mathrm{Al}_{2} \mathrm{O}_{3}$ & & 7200 & & 100 & 450 & 54 & & & 0.093 & 32 \\
\hline $\mathrm{Ru}$ & 5 & $\mathrm{Al}_{2} \mathrm{O}_{3}$ & & & 13000 & 10 & 400 & 79 & & & & 224 \\
\hline $\mathrm{Ru}$ & 8.5 & $\mathrm{Al}_{2} \mathrm{O}_{3}$ & & & 1200 & 100 & 400 & 99 & & 117 & & 74 \\
\hline $\mathrm{Ru}$ & 10 & $\mathrm{Al}_{2} \mathrm{O}_{3}$ & & 30000 & & 100 & 450 & & & 80 & 6.5 & 158 \\
\hline $\mathrm{Ru}$ & 4 & $\mathrm{Al}_{2} \mathrm{O}_{3}$ & $\mathrm{Ba}$ & & & 10 & 400 & 37 & & & & 190 \\
\hline $\mathrm{Ru}$ & 4 & $\mathrm{Al}_{2} \mathrm{O}_{3}$ & $\mathrm{Ba}, \mathrm{K}$ & & & 10 & 400 & 95 & & & & 190 \\
\hline $\mathrm{Ru}$ & 4 & $\mathrm{Al}_{2} \mathrm{O}_{3}$ & Cs & & & 10 & 400 & 47 & & & & 190 \\
\hline $\mathrm{Ru}$ & 4.7 & $\mathrm{Al}_{2} \mathrm{O}_{3}$ & Cs & & & 67 & 350 & & & & 0.10 & 189 \\
\hline $\mathrm{Ru}$ & 4 & $\mathrm{Al}_{2} \mathrm{O}_{3}$ & $\mathrm{~K}$ & & & 10 & 400 & 96 & & & & 190 \\
\hline $\mathrm{Ru}$ & 4 & $\mathrm{Al}_{2} \mathrm{O}_{3}$ & $\mathrm{~K}$ & 5400 & & 100 & 400 & & & 66 & 0.33 & 222 \\
\hline $\mathrm{Ru}$ & 4.6 & $\mathrm{Al}_{2} \mathrm{O}_{3}$ & $\mathrm{~K}$ & 30000 & & 100 & 400 & 12 & 0.07 & & 0.19 & 195 \\
\hline $\mathrm{Ru}$ & 4.7 & $\mathrm{Al}_{2} \mathrm{O}_{3}$ & K & & & 67 & 350 & & & & 0.12 & 189 \\
\hline $\mathrm{Ru}$ & 5 & $\mathrm{Al}_{2} \mathrm{O}_{3}$ & $\mathrm{~K}$ & 30000 & & 100 & 550 & 86 & & & & 223 \\
\hline $\mathrm{Ru}$ & 4.7 & $\mathrm{Al}_{2} \mathrm{O}_{3}$ & $\mathrm{Li}$ & 3700 & & 67 & 350 & & & & 0.26 & 189 \\
\hline $\mathrm{Ru}$ & 1 & $\mathrm{Al}_{2} \mathrm{O}_{3}$ & $\mathrm{LiOH}$ & & & 100 & 350 & & & & 1.6 & 225 \\
\hline $\mathrm{Ru}$ & 7 & $\mathrm{Al}_{2} \mathrm{O}_{3}$ rods & & 6000 & & 29 & 350 & 4 & & 111 & 0.006 & 211 \\
\hline $\mathrm{Ru}$ & 7 & $\mathrm{Al}_{2} \mathrm{O}_{3}$ rods & $\mathrm{Na}$ & 6000 & & 29 & 350 & 21 & & 95 & 0.031 & 211 \\
\hline $\mathrm{Ru}$ & 7.0 & $\mathrm{AX}-21$ & & 5200 & 33 & 100 & 450 & 24 & & & & 226 \\
\hline $\mathrm{Ru}$ & 3 & $\mathrm{Ba}-\mathrm{ZrO}_{2}$ & & 30000 & & 100 & 500 & 24 & 0.13 & 92 & & 197 \\
\hline $\mathrm{Ru}$ & 3 & $\mathrm{Ba}-\mathrm{ZrO}_{2}$ & Cs & 30000 & & 100 & 500 & 38 & 0.21 & 64 & & 197 \\
\hline $\mathrm{Ru}$ & 3 & $\mathrm{Ba}-\mathrm{ZrO}_{2}$ & $\mathrm{~K}$ & 30000 & & 100 & 500 & 33 & 0.18 & 71 & & 197 \\
\hline $\mathrm{Ru}$ & 2.7 & BHA & & 30000 & & 100 & 450 & 42 & & 65 & 2.9 & 214 \\
\hline $\mathrm{Ru}$ & 5 & black carbon & & 30000 & & 100 & 550 & 56 & 0.27 & & & 183 \\
\hline $\mathrm{Ru}$ & 9.1 & $\mathrm{C}$ & & 1500000 & & 5.7 & 400 & & & 191 & & 185 \\
\hline
\end{tabular}


Table 2. continued

\begin{tabular}{|c|c|c|c|c|c|c|c|c|c|c|c|c|}
\hline $\begin{array}{l}\text { active } \\
\text { phase }\end{array}$ & wt $\%$ & support & promoter & $\begin{array}{c}\text { WHSV } \\
\left(\mathrm{mL} \mathrm{g}^{-1} \mathrm{~h}^{-1}\right)\end{array}$ & $\begin{array}{c}\text { GHSV } \\
\left(\mathrm{h}^{-1}\right)\end{array}$ & $\begin{array}{c}\% \mathrm{NH}_{3} \\
\text { inlet flow }\end{array}$ & $\begin{array}{l}T\left(^{\circ}\right. \\
\mathrm{C})\end{array}$ & $\begin{array}{l}\text { conv } \\
(\%)\end{array}$ & $\begin{array}{c}\mathrm{H}_{2} \text { rate } \\
\left(\mathrm{mmol} \mathrm{g}^{-1} \mathrm{~s}^{-1}\right)\end{array}$ & $\begin{array}{c}E_{\mathrm{a}} \\
\left(\mathrm{kJ} \mathrm{\textrm {mol } ^ { - 1 }}\right)\end{array}$ & $\begin{array}{l}\text { TOF } \\
\left(s^{-1}\right)\end{array}$ & ref \\
\hline $\mathrm{Ru}$ & 9.1 & $\mathrm{C}$ & & & & 50 & 400 & & & & 0.5 & 227 \\
\hline $\mathrm{Ru}$ & 25 & $\mathrm{C}$ & & 30000 & & 100 & 400 & 42 & 0.23 & 54 & & 186 \\
\hline $\mathrm{Ru}$ & 4.6 & $\mathrm{C}$ & $\mathrm{Ba}$ & & & 20 & 400 & & & 158 & 1.64 & 194 \\
\hline $\mathrm{Ru}$ & 9.1 & $\mathrm{C}$ & $\mathrm{Ba}$ & 1500000 & & 5.7 & 400 & & & 158 & 0.56 & 185 \\
\hline $\mathrm{Ru}$ & & $\mathrm{C}$ & Cs & 30000 & & 100 & 400 & 90 & 0.50 & & & 186 \\
\hline $\mathrm{Ru}$ & 4.6 & $\mathrm{C}$ & Cs & & & 20 & 400 & & & 134 & 5.10 & 194 \\
\hline $\mathrm{Ru}$ & 9.1 & $\mathrm{C}$ & Cs & 1500000 & & 5.7 & 400 & & & 134 & 1.48 & 185 \\
\hline $\mathrm{Ru}$ & 2.7 & $\mathrm{C}$ & $\mathrm{K}$ & 15000 & & 30 & 400 & 56 & & 57 & 2.9 & 213 \\
\hline $\mathrm{Ru}$ & 9.1 & $\mathrm{C}$ & K & & & 50 & 400 & & & 139 & 34.7 & 170 \\
\hline $\mathrm{Ru}$ & 3.5 & $\mathrm{CaAlO}_{x}$ & & 6000 & & 100 & 450 & 78 & & & & 212 \\
\hline $\mathrm{Ru}$ & 1.8 & $\mathrm{CaO}$ & & 15000 & & 30 & 400 & 15 & & 84 & 2.6 & 213 \\
\hline $\mathrm{Ru}$ & 2.9 & $\mathrm{CaO}$ & & 9000 & & 100 & 400 & 7 & & 96 & 0.20 & 73 \\
\hline $\mathrm{Ru}$ & 2.8 & $\mathrm{CaO}$ & $\mathrm{K}$ & 9000 & & 100 & 400 & 60 & & 75 & 1.25 & 73 \\
\hline $\mathrm{Ru}$ & 2 & $\mathrm{CeO}_{2}$ & & 13800 & 3067 & 57 & 400 & 77 & & & 1.8 & 219 \\
\hline $\mathrm{Ru}$ & 1 & $\mathrm{CeO}_{2}$ rods & & 22000 & & 100 & 350 & 32 & & 151 & 3.2 & 210 \\
\hline $\mathrm{Ru}$ & 7 & $\mathrm{CeO}_{2}$ rods & & 6000 & & 29 & 350 & 23 & & 83 & 0.034 & 211 \\
\hline $\mathrm{Ru}$ & 7 & $\mathrm{CeO}_{2}$ rods & $\mathrm{Na}$ & 6000 & & 29 & 350 & 25 & & 86 & 0.046 & 211 \\
\hline $\mathrm{Ru}$ & 1 & $\mathrm{CeO}_{2}$ spheres & & 22000 & & 100 & 350 & 25 & & & & 210 \\
\hline $\mathrm{Ru}$ & 5 & CMK-3 & & 30000 & & 100 & 550 & 23 & 0.12 & & & 183 \\
\hline $\mathrm{Ru}$ & 5 & CMK-3 & & 30000 & & 100 & 550 & 23 & 0.12 & & & 187 \\
\hline $\mathrm{Ru}$ & 5 & CMK-3 & $\mathrm{Ca}$ & 30000 & & 100 & 550 & 49 & 0.25 & & & 187 \\
\hline $\mathrm{Ru}$ & 5 & CMK-3 & $\mathrm{Cl}$ & 30000 & & 100 & 550 & 37 & 0.19 & & & 187 \\
\hline $\mathrm{Ru}$ & 5 & CMK-3 & $\mathrm{K}$ & 30000 & & 100 & 550 & 79 & 0.40 & & & 187 \\
\hline $\mathrm{Ru}$ & 5 & CMK-3 & $\mathrm{Li}$ & 30000 & & 100 & 550 & 15 & 0.08 & & & 187 \\
\hline $\mathrm{Ru}$ & 5 & CMK-3 & $\mathrm{Na}$ & 30000 & & 100 & 550 & 51 & 0.26 & & & 187 \\
\hline $\mathrm{Ru}$ & 5 & CMK-3 & $\mathrm{PO}_{4}$ & 30000 & & 100 & 550 & 34 & 0.17 & & & 187 \\
\hline $\mathrm{Ru}$ & 5 & CMK-3 & $\mathrm{SO}_{4}$ & 30000 & & 100 & 550 & 36 & 0.18 & & & 187 \\
\hline $\mathrm{Ru}$ & 3.9 & CNFs & & 9900 & & 5 & 450 & 72 & & & & 175 \\
\hline $\mathrm{Ru}$ & 7.9 & CNFs & & 6500 & & 100 & 400 & 55 & & 183 & 0.05 & 176 \\
\hline $\mathrm{Ru}$ & 10 & CNFs & & 36000 & & 100 & 500 & & & & 0.2 & 228 \\
\hline $\mathrm{Ru}$ & 0.8 & CNTs & & 42000 & & 100 & 550 & 44 & & & 22.5 & 174 \\
\hline $\mathrm{Ru}$ & 1.7 & CNTs & & 25200 & & 100 & 500 & 17 & & & & 229 \\
\hline $\mathrm{Ru}$ & 2 & CNTs & & 20000 & & 10 & 400 & 18 & & & & 173 \\
\hline $\mathrm{Ru}$ & 2 & CNTs & & 36000 & & 100 & 450 & & 0.18 & & & 230 \\
\hline $\mathrm{Ru}$ & 2 & CNTs & & 6000 & & 100 & 450 & 82 & & & & 231 \\
\hline $\mathrm{Ru}$ & 2.5 & CNTs & & 30000 & & 100 & 450 & 18 & & 87 & 1.1 & 214 \\
\hline $\mathrm{Ru}$ & 3.2 & CNTs & & 6500 & & 100 & 400 & 8 & & & & 176 \\
\hline $\mathrm{Ru}$ & 3.5 & CNTs & & 6000 & & 100 & 450 & 21 & & & & 212 \\
\hline $\mathrm{Ru}$ & 4.8 & CNTs & & 60000 & & 100 & 400 & 12 & & 71 & & 169 \\
\hline $\mathrm{Ru}$ & 4.8 & CNTs & & 30000 & & 100 & 450 & & 0.24 & 69 & 2.3 & 180 \\
\hline $\mathrm{Ru}$ & 4.85 & CNTs & & 60000 & & 100 & 400 & 9 & 0.08 & & & 188 \\
\hline $\mathrm{Ru}$ & 5 & CNTs & & 30000 & & 100 & 500 & 88 & 0.47 & 69 & & 156 \\
\hline $\mathrm{Ru}$ & 5.0 & CNTs & & 6000 & & 100 & 450 & 69 & & & & 171 \\
\hline $\mathrm{Ru}$ & 5 & CNTs & & 30000 & & 100 & 550 & 85 & 0.43 & & & 183 \\
\hline $\mathrm{Ru}$ & 5 & CNTs & & 60000 & & 5 & 400 & 84 & & 89 & & 232 \\
\hline $\mathrm{Ru}$ & 5 & CNTs & & & & & & & & & & 233 \\
\hline $\mathrm{Ru}$ & 7 & CNTs & & 5200 & & 100 & 327 & 2 & & 97 & 0.002 & 168 \\
\hline $\mathrm{Ru}$ & 7 & CNTs & & 20000 & & 29 & 400 & 34 & & 81 & 0.05 & 172 \\
\hline $\mathrm{Ru}$ & 7 & CNTs & & 6000 & & 29 & 350 & 2 & & 104 & 0.004 & 211 \\
\hline $\mathrm{Ru}$ & 7.0 & CNTs & & 5200 & 33 & 100 & 450 & 50 & & & & 226 \\
\hline $\mathrm{Ru}$ & 4.8 & CNTs & $\mathrm{Ba}$ & 60000 & & 100 & 400 & 20 & & 65 & & 169 \\
\hline $\mathrm{Ru}$ & 4.8 & CNTs & $\mathrm{Ca}$ & 60000 & & 100 & 400 & 15 & & 70 & & 169 \\
\hline $\mathrm{Ru}$ & 4.8 & CNTs & $\mathrm{Ce}$ & 60000 & & 100 & 400 & 26 & & 63 & & 169 \\
\hline $\mathrm{Ru}$ & 7 & CNTs & Cs & 5200 & & & 327 & 46 & & 60 & 0.04 & 167 \\
\hline $\mathrm{Ru}$ & 7 & CNTs & Cs & 5200 & & 100 & 327 & 14 & & 59 & 0.04 & 168 \\
\hline $\mathrm{Ru}$ & 4.8 & CNTs & $\mathrm{K}$ & 60000 & & 100 & 400 & 63 & & 54 & 4.8 & 169 \\
\hline $\mathrm{Ru}$ & 4.8 & CNTs & $\mathrm{K}$ & 30000 & & 100 & 450 & & 0.54 & 56 & 5.1 & 180 \\
\hline $\mathrm{Ru}$ & 4.85 & CNTs & $\mathrm{K}$ & 60000 & & 100 & 400 & 62 & 0.70 & & & 188 \\
\hline $\mathrm{Ru}$ & 5 & CNTs & $\mathrm{K}$ & 150000 & & 100 & 500 & 28 & 0.80 & 56 & & 156 \\
\hline $\mathrm{Ru}$ & 4.8 & CNTs & $\mathrm{La}$ & 60000 & & 100 & 400 & 17 & & 67 & & 169 \\
\hline
\end{tabular}


Table 2. continued

\begin{tabular}{|c|c|c|c|c|c|c|c|c|c|c|c|c|}
\hline $\begin{array}{l}\text { active } \\
\text { phase }\end{array}$ & wt $\%$ & support & promoter & $\begin{array}{c}\text { WHSV } \\
\left(\mathrm{mL} \mathrm{g}^{-1} \mathrm{~h}^{-1}\right)\end{array}$ & $\begin{array}{c}\text { GHSV } \\
\left(h^{-1}\right)\end{array}$ & $\begin{array}{c}\% \mathrm{NH}_{3} \\
\text { inlet flow }\end{array}$ & $\begin{array}{l}T\left(^{\circ}\right. \\
\mathrm{C})\end{array}$ & $\begin{array}{l}\text { conv } \\
(\%)\end{array}$ & $\begin{array}{c}\mathrm{H}_{2} \text { rate } \\
\left(\mathrm{mmol} \mathrm{g}^{-1} \mathrm{~s}^{-1}\right)\end{array}$ & $\begin{array}{c}E_{\mathrm{a}} \\
\left(\mathrm{kJ} \mathrm{mol}^{-1}\right)\end{array}$ & $\begin{array}{l}\text { TOF } \\
\left(s^{-1}\right)\end{array}$ & ref \\
\hline $\mathrm{Ru}$ & 4.8 & CNTs & $\mathrm{Li}$ & 60000 & & 100 & 400 & 33 & & 64 & & 169 \\
\hline $\mathrm{Ru}$ & 4.8 & CNTs & $\mathrm{Na}$ & 60000 & & 100 & 400 & 41 & & 61 & & 169 \\
\hline $\mathrm{Ru}$ & 7 & CNTs & $\mathrm{Na}$ & 6000 & & 29 & 350 & 58 & & 85 & 0.088 & 211 \\
\hline $\mathrm{Ru}$ & 4.85 & CNTs-MgO & & 60000 & & 100 & 400 & 13 & 0.10 & & & 188 \\
\hline $\mathrm{Ru}$ & 4.85 & CNTs-MgO & $\mathrm{K}$ & 60000 & & 100 & 400 & 80 & 0.88 & & & 188 \\
\hline $\mathrm{Ru}$ & 5 & $\mathrm{Cr}_{2} \mathrm{O}_{3}$ & & 30000 & & 100 & 450 & 11 & & 76 & 3.5 & 234 \\
\hline $\mathrm{Ru}$ & 3 & $\mathrm{Cs}_{2} \mathrm{Ti}_{6} \mathrm{O}_{13}$ & & 42000 & & 29 & & & & & & 191 \\
\hline $\mathrm{Ru}$ & 4.8 & $\mathrm{Er}_{2} \mathrm{O}_{3}$ & & 18000 & & 100 & 350 & 11 & & & & 193 \\
\hline $\mathrm{Ru}$ & 5 & fly ashes & & 60000 & & 100 & 550 & 7 & 0.07 & & & 205 \\
\hline $\mathrm{Ru}$ & 5 & fumed $\mathrm{SiO}_{2}$ & & 30000 & & 100 & 500 & 26 & & & & 164 \\
\hline $\mathrm{Ru}$ & 5 & fumed $\mathrm{SiO}_{2}$ & $\mathrm{~K}$ & 30000 & & 100 & 500 & 28 & & & & 164 \\
\hline $\mathrm{Ru}$ & 5 & GC & & 30000 & & 100 & 550 & 95 & 0.49 & & & 183 \\
\hline $\mathrm{Ru}$ & 5 & GNP & & 15000 & 12000 & 10 & 375 & 35 & & 73 & & 235 \\
\hline $\mathrm{Ru}$ & 5 & GNP & $\mathrm{K}$ & 15000 & 12000 & 10 & 375 & 62 & & & & 235 \\
\hline $\mathrm{Ru}$ & 5 & GNP & $\mathrm{Na}$ & 15000 & 12000 & 10 & 375 & 44 & & & & 235 \\
\hline $\mathrm{Ru}$ & & graphene & & 20000 & & 100 & 450 & 91 & 0.35 & & & 182 \\
\hline $\mathrm{Ru}$ & 2 & graphite & & 36000 & & 100 & 600 & 93 & & & & 236 \\
\hline $\mathrm{Ru}$ & 7 & graphitized CNTs & & 5200 & & 100 & 327 & 14 & & 68 & 0.01 & 168 \\
\hline $\mathrm{Ru}$ & 7 & graphitized CNTs & Cs & 5200 & & 100 & 327 & 72 & & 54 & 0.07 & 168 \\
\hline $\mathrm{Ru}$ & 2.6 & HNTs & & 12600 & & 100 & 525 & 81 & & & 8.75 & 209 \\
\hline $\mathrm{Ru}$ & 3 & $\mathrm{~K}_{2} \mathrm{Ti}_{6} \mathrm{O}_{13}$ & & 42000 & & 29 & & & & & & 191 \\
\hline $\mathrm{Ru}$ & 4.8 & $\mathrm{La}_{2} \mathrm{O}_{3}$ & & 18000 & & 100 & 350 & 19 & & 42 & 0.43 & 193 \\
\hline $\mathrm{Ru}$ & 5 & $\mathrm{La}_{2} \mathrm{O}_{3}$ & & 3000 & & 100 & 350 & 40 & & & & 216 \\
\hline $\mathrm{Ru}$ & 5 & $\mathrm{La}_{2} \mathrm{O}_{3}$ & $\mathrm{Cs}_{2} \mathrm{O}$ & 3000 & & 100 & 350 & 73 & & & & 216 \\
\hline $\mathrm{Ru}$ & 4.8 & $\mathrm{La}_{2} \mathrm{O}_{3}$ & $\mathrm{~K}$ & 18000 & & 100 & 350 & 33 & & 35 & 3.66 & 193 \\
\hline $\mathrm{Ru}$ & 3 & $\mathrm{La}_{2} \mathrm{O}_{3}-\mathrm{ZrO}_{2}$ & & 4000 & & 100 & 450 & 4 & & & & 198 \\
\hline $\mathrm{Ru}$ & 3 & $\mathrm{La}_{2} \mathrm{O}_{3}-\mathrm{ZrO}_{2}$ & $\mathrm{NaBH}_{4}$ & 4000 & & 100 & 450 & 7 & & & & 198 \\
\hline $\mathrm{Ru}$ & 0.7 & $\mathrm{La}-\mathrm{Al}_{2} \mathrm{O}_{3}$ & & 2300 & & 10 & 500 & 98 & & 72 & & 192 \\
\hline $\mathrm{Ru}$ & 5 & MCM-41 & & 30000 & & 100 & 500 & 24 & & & & 164 \\
\hline $\mathrm{Ru}$ & 5 & MCM-41 & $\mathrm{K}$ & 30000 & & 100 & 500 & 29 & & 50 & 7.2 & 164 \\
\hline $\mathrm{Ru}$ & 4.6 & $\mathrm{MgAl}_{2} \mathrm{O}_{4}$ & $\mathrm{Ba}$ & & & 20 & 400 & & & 124 & 0.46 & 194 \\
\hline $\mathrm{Ru}$ & 4.6 & $\mathrm{MgAl}_{2} \mathrm{O}_{4}$ & Cs & & & 20 & 400 & & & 105 & 0.54 & 194 \\
\hline $\mathrm{Ru}$ & 1 & $\mathrm{MgO}$ & & 22000 & & 100 & 350 & 11 & & & 0.6 & 210 \\
\hline $\mathrm{Ru}$ & 1 & $\mathrm{MgO}$ & & 3000 & & 100 & 350 & 11 & & & & 216 \\
\hline $\mathrm{Ru}$ & 2.0 & $\mathrm{MgO}$ & & 15000 & & 30 & 400 & 33 & & 82 & 1.5 & 213 \\
\hline $\mathrm{Ru}$ & 2.8 & $\mathrm{MgO}$ & & 30000 & & 100 & 450 & 41 & & & 7 & 201 \\
\hline $\mathrm{Ru}$ & 2.9 & $\mathrm{MgO}$ & & 30000 & & 100 & 450 & 12 & & 103 & 1.6 & 214 \\
\hline $\mathrm{Ru}$ & 3.5 & $\mathrm{MgO}$ & & 36000 & & 100 & 450 & 53 & 0.35 & 83 & 3 & 202 \\
\hline $\mathrm{Ru}$ & 4.7 & $\mathrm{MgO}$ & & 60000 & & 100 & 400 & 6 & 0.07 & 99 & 0.33 & 237 \\
\hline $\mathrm{Ru}$ & 4.8 & $\mathrm{MgO}$ & & 30000 & & 100 & 450 & & 0.17 & 62 & 4.0 & 180 \\
\hline $\mathrm{Ru}$ & 4.85 & $\mathrm{MgO}$ & & 60000 & & 100 & 400 & 7 & 0.07 & & & 188 \\
\hline $\mathrm{Ru}$ & 5 & $\mathrm{MgO}$ & & & 15000 & 100 & 450 & 16 & & 124 & & 238 \\
\hline $\mathrm{Ru}$ & 5 & $\mathrm{MgO}$ & & 150000 & & 100 & 500 & 13 & 0.37 & 62 & & 156 \\
\hline $\mathrm{Ru}$ & 5 & $\mathrm{MgO}$ & & 60000 & & 5 & 400 & 47 & & 86 & & 232 \\
\hline $\mathrm{Ru}$ & 2.0 & $\mathrm{MgO}$ & Cs & 15000 & & 30 & 400 & 53 & & 68 & 2.5 & 213 \\
\hline $\mathrm{Ru}$ & 2.8 & $\mathrm{MgO}$ & Cs & 30000 & & 100 & 450 & 61 & & & & 201 \\
\hline $\mathrm{Ru}$ & 2.8 & $\mathrm{MgO}$ & $\mathrm{K}$ & 30000 & & 100 & 450 & 57 & & & & 201 \\
\hline $\mathrm{Ru}$ & 3.5 & $\mathrm{MgO}$ & $\mathrm{K}$ & 36000 & & 100 & 450 & 87 & 0.47 & 58 & & 202 \\
\hline $\mathrm{Ru}$ & 4.6 & $\mathrm{MgO}$ & $\mathrm{K}$ & 30000 & & 100 & 400 & 11 & 0.06 & & 0.31 & 195 \\
\hline $\mathrm{Ru}$ & 4.8 & $\mathrm{MgO}$ & $\mathrm{K}$ & 9000 & & 100 & 400 & 39 & 0.11 & & & 73 \\
\hline $\mathrm{Ru}$ & 4.85 & $\mathrm{MgO}$ & $\mathrm{K}$ & 60000 & & 100 & 400 & 22 & 0.25 & & & 188 \\
\hline $\mathrm{Ru}$ & 5 & $\mathrm{MgO}$ & $\mathrm{K}$ & 30000 & & 100 & 450 & 29 & & & & 179 \\
\hline $\mathrm{Ru}$ & 5 & $\mathrm{MgO}$ & $\mathrm{K}$ & 60000 & & 5 & 400 & 76 & & 51 & & 232 \\
\hline $\mathrm{Ru}$ & 2 & $\mathrm{MgO}-\mathrm{Al}_{2} \mathrm{O}_{3}$ & & 30000 & & 100 & 500 & 86 & & & & 196 \\
\hline $\mathrm{Ru}$ & & MgO-MIL-101 & & 15000 & & 100 & 400 & 45 & & & & 203 \\
\hline $\mathrm{Ru}$ & & MgO-MIL-101 & Cs & 15000 & & 100 & 400 & 88 & & & & 203 \\
\hline $\mathrm{Ru}$ & 4.6 & $\mathrm{Mg}_{y} \mathrm{Al}_{z} \mathrm{O}_{\mathrm{n}}$ & $\mathrm{K}$ & 30000 & & 100 & 400 & 17 & 0.10 & & 0.61 & 195 \\
\hline $\mathrm{Ru}$ & & MIL-101 & & 15000 & & 100 & 400 & 42 & & & & 203 \\
\hline $\mathrm{Ru}$ & & MIL-101 & Cs & 15000 & & 100 & 400 & 66 & & & & 203 \\
\hline $\mathrm{Ru}$ & 4.7 & MWCNT & $\mathrm{K}$ & 9000 & & 100 & 400 & 31 & 0.09 & & & 73 \\
\hline
\end{tabular}


Table 2. continued

\begin{tabular}{|c|c|c|c|c|c|c|c|c|c|c|c|c|}
\hline $\begin{array}{l}\text { active } \\
\text { phase }\end{array}$ & wt $\%$ & support & promoter & $\begin{array}{c}\text { WHSV } \\
\left(\mathrm{mL} \mathrm{g}^{-1} \mathrm{~h}^{-1}\right)\end{array}$ & $\begin{array}{c}\text { GHSV } \\
\left(\mathrm{h}^{-1}\right)\end{array}$ & $\begin{array}{c}\% \mathrm{NH}_{3} \\
\text { inlet flow }\end{array}$ & $\begin{array}{l}T\left(^{\circ}\right. \\
\mathrm{C})\end{array}$ & $\begin{array}{l}\text { conv } \\
(\%)\end{array}$ & $\begin{array}{c}\mathrm{H}_{2} \text { rate } \\
\left(\mathrm{mmol} \mathrm{g}^{-1} \mathrm{~s}^{-1}\right)\end{array}$ & $\begin{array}{c}E_{\mathrm{a}} \\
\left(\mathrm{kJ} \mathrm{mol}^{-1}\right)\end{array}$ & $\begin{array}{l}\text { TOF } \\
\left(s^{-1}\right)\end{array}$ & ref \\
\hline $\mathrm{Ru}$ & 3 & $\mathrm{Na}_{2} \mathrm{Ti}_{3} \mathrm{O}_{7}$ & & 42000 & & 29 & & & & & & 191 \\
\hline $\mathrm{Ru}$ & 5.1 & N-CNFs & & 9900 & & 5 & 450 & 86 & & & & 175 \\
\hline $\mathrm{Ru}$ & 0.8 & N-CNTs & & 42000 & & 100 & 550 & 61 & & & 18.8 & 174 \\
\hline $\mathrm{Ru}$ & 2 & N-CNTs & & 20000 & & 10 & 400 & 37 & & & & 173 \\
\hline $\mathrm{Ru}$ & 7 & $\mathrm{~N}-\mathrm{CNTs}$ & & 20000 & & 29 & 400 & 48 & & 85 & 0.07 & 172 \\
\hline $\mathrm{Ru}$ & 5.0 & N-OMC & & 6000 & & 100 & 450 & 85 & & 37 & & 171 \\
\hline $\mathrm{Ru}$ & 3.7 & $\mathrm{O}-\mathrm{CNFs}$ & & 9900 & & 5 & 450 & 69 & & & & 175 \\
\hline $\mathrm{Ru}$ & 5.0 & OMC & & 6000 & & 100 & 450 & 59 & & & & 171 \\
\hline $\mathrm{Ru}$ & 5 & $\mathrm{Pr}_{6} \mathrm{O}_{11}$ & & 3000 & & 100 & 350 & 41 & & & & 215 \\
\hline $\mathrm{Ru}$ & 5 & $\operatorname{Pr}_{6} \mathrm{O}_{11}$ & $\mathrm{BaO}$ & 3000 & & 100 & 350 & 20 & & & & 215 \\
\hline $\mathrm{Ru}$ & 5 & $\operatorname{Pr}_{6} \mathrm{O}_{11}$ & $\mathrm{CaO}$ & 3000 & & 100 & 350 & 19 & & & & 215 \\
\hline $\mathrm{Ru}$ & 5 & $\mathrm{Pr}_{6} \mathrm{O}_{11}$ & $\mathrm{Cs}_{2} \mathrm{O}$ & 3000 & & 100 & 350 & 93 & & & 0.3 & 215 \\
\hline $\mathrm{Ru}$ & 5 & $\operatorname{Pr}_{6} \mathrm{O}_{11}$ & $\mathrm{Gd}_{2} \mathrm{O}_{3}$ & 3000 & & 100 & 350 & 25 & & & & 215 \\
\hline $\mathrm{Ru}$ & 5 & $\mathrm{Pr}_{6} \mathrm{O}_{11}$ & $\mathrm{~K}_{2} \mathrm{O}$ & 3000 & & 100 & 350 & 61 & & & & 215 \\
\hline $\mathrm{Ru}$ & 5 & $\operatorname{Pr}_{6} \mathrm{O}_{11}$ & $\mathrm{La}_{2} \mathrm{O}_{3}$ & 3000 & & 100 & 350 & 22 & & & & 215 \\
\hline $\mathrm{Ru}$ & 5 & $\mathrm{Pr}_{6} \mathrm{O}_{11}$ & $\mathrm{Li}_{2} \mathrm{O}$ & 3000 & & 100 & 350 & 36 & & & & 215 \\
\hline $\mathrm{Ru}$ & 5 & $\mathrm{Pr}_{6} \mathrm{O}_{11}$ & $\mathrm{MgO}$ & 3000 & & 100 & 350 & 25 & & & & 215 \\
\hline $\mathrm{Ru}$ & 5 & $\mathrm{Pr}_{6} \mathrm{O}_{11}$ & $\mathrm{Na}_{2} \mathrm{O}$ & 3000 & & 100 & 350 & 49 & & & & 215 \\
\hline $\mathrm{Ru}$ & 5 & $\mathrm{Pr}_{6} \mathrm{O}_{11}$ & $\mathrm{Pr}_{6} \mathrm{O}_{11}$ & 3000 & & 100 & 350 & 27 & & & & 215 \\
\hline $\mathrm{Ru}$ & 5 & $\mathrm{Pr}_{6} \mathrm{O}_{11}$ & $\mathrm{Rb}_{2} \mathrm{O}$ & 3000 & & 100 & 350 & 66 & & & & 215 \\
\hline $\mathrm{Ru}$ & 5 & $\mathrm{Pr}_{6} \mathrm{O}_{11}$ & $\mathrm{Sm}_{2} \mathrm{O}_{3}$ & 3000 & & 100 & 350 & 21 & & & & 215 \\
\hline $\mathrm{Ru}$ & 5 & $\mathrm{Pr}_{6} \mathrm{O}_{11}$ & $\mathrm{SrO}$ & 3000 & & 100 & 350 & 21 & & & & 215 \\
\hline $\mathrm{Ru}$ & 5 & $\mathrm{Pr}_{6} \mathrm{O}_{11}$ & $\mathrm{Yb}_{2} \mathrm{O}_{3}$ & 3000 & & 100 & 350 & 20 & & & & 215 \\
\hline $\mathrm{Ru}$ & 5 & red mud & & 60000 & & 100 & 550 & 17 & 0.19 & & & 205 \\
\hline $\mathrm{Ru}$ & 5.5 & red mud & & 240000 & & 100 & 550 & 24 & & 92 & & 204 \\
\hline $\mathrm{Ru}$ & 5 & SBA-15 & & 30000 & & 100 & 500 & 25 & & & & 164 \\
\hline $\mathrm{Ru}$ & 5 & SBA-15 & $\mathrm{K}$ & 30000 & & 100 & 500 & 27 & & & & 164 \\
\hline $\mathrm{Ru}$ & 4.9 & Sepiolite & $\mathrm{K}$ & 9000 & & 100 & 400 & 47 & 0.13 & & & 73 \\
\hline $\mathrm{Ru}$ & & Sibunit & & 34200 & 17100 & 100 & 450 & 13 & & & & 181 \\
\hline $\mathrm{Ru}$ & & Sibunit & $\mathrm{K}$ & 34200 & 17100 & 100 & 450 & 23 & & & & 181 \\
\hline $\mathrm{Ru}$ & 4.8 & $\mathrm{SiO}_{2}$ & & 18000 & & 100 & 350 & 4 & & & & 193 \\
\hline $\mathrm{Ru}$ & 5 & $\mathrm{SiO}_{2}$ & & 6000 & & 100 & 450 & 62 & & & & 212 \\
\hline $\mathrm{Ru}$ & 10 & $\mathrm{SiO}_{2}$ & & 30000 & & 100 & 450 & 36 & 0.19 & 82 & 10.5 & 158 \\
\hline $\mathrm{Ru}$ & 1 & $\mathrm{SiO}_{2}-\mathrm{Al}_{2} \mathrm{O}_{3}$ & & 3000 & & 100 & 350 & 3 & & & & 216 \\
\hline $\mathrm{Ru}$ & 3 & $\mathrm{TiO}_{2}$ & & 42000 & & 29 & & & & & & 191 \\
\hline $\mathrm{Ru}$ & 4.8 & $\mathrm{TiO}_{2}$ & & 30000 & & 100 & 450 & & 0.15 & 61 & 3.1 & 180 \\
\hline $\mathrm{Ru}$ & 4.8 & $\mathrm{TiO}_{2}$ & & 18000 & & 100 & 350 & 1 & & & & 193 \\
\hline $\mathrm{Ru}$ & 5 & $\mathrm{TiO}_{2}$ & & 150000 & & 100 & 500 & 12 & 0.32 & 63 & & 156 \\
\hline $\mathrm{Ru}$ & 7 & titanate NTs & $\mathrm{Na}$ & 6000 & & 29 & 350 & 6 & & 96 & 0.009 & 211 \\
\hline $\mathrm{Ru}$ & 0.5 & $\mathrm{ZrO}_{2}$ & & 4600 & & 100 & 500 & 60 & & & 0.025 & 199 \\
\hline $\mathrm{Ru}$ & 3 & $\mathrm{ZrO}_{2}$ & & 3000 & & 100 & 500 & 43 & & & & 197 \\
\hline $\mathrm{Ru}$ & 5 & $\mathrm{ZrO}_{2}$ & & 150000 & & 100 & 500 & 10 & 0.27 & 66 & & 156 \\
\hline $\mathrm{Ru}$ & 3 & $\mathrm{ZrO}_{2}$ & $\mathrm{Ba}$ & 3000 & & 100 & 500 & 29 & & & & 197 \\
\hline $\mathrm{Ru}$ & 0.5 & $\mathrm{ZrO}_{2}$ & $\mathrm{Ca}, \mathrm{La}$ & 4600 & & 100 & 500 & 87 & & & 0.038 & 199 \\
\hline $\mathrm{Ru}$ & 5 & $\mathrm{ZrO}_{2}$ & $\mathrm{~K}$ & 150000 & & 100 & 500 & 19 & 0.53 & 57 & & 156 \\
\hline $\mathrm{Ru}$ & 0.5 & $\mathrm{ZrO}_{2}$ & $\mathrm{La}$ & 4600 & & 100 & 500 & 86 & & & 0.039 & 199 \\
\hline $\mathrm{Ru}$ & 0.5 & $\mathrm{ZrO}_{2}$ & $\mathrm{Mg}, \mathrm{La}$ & 4600 & & 100 & 500 & 84 & & & 0.031 & 199 \\
\hline $\mathrm{Ru}$ & 0.5 & $\mathrm{ZrO}_{2}$ & $\mathrm{Sr}, \mathrm{La}$ & 4600 & & 100 & 500 & 76 & & & 0.046 & 199 \\
\hline $\mathrm{Ru}$ & 4.85 & $\mathrm{ZrO}_{2}-\mathrm{KOH}$ & & 150000 & & 100 & 350 & 18 & 0.49 & 47 & 4.9 & 200 \\
\hline $\mathrm{Ru}$ & 4.85 & $\mathrm{ZrO}_{2}-\mathrm{KOH}$ & $\mathrm{K}$ & 150000 & & 100 & 350 & 15 & 0.41 & 48 & 4.7 & 200 \\
\hline
\end{tabular}

catalytic activity compared to $\mathrm{Ru} / \mathrm{Pr}_{6} \mathrm{O}_{11}$ and $\mathrm{Ru} / \mathrm{La}_{2} \mathrm{O}_{3}{ }^{216}$ Catalysts based on ruthenium that are used to decompose ammonia and their catalytic performances are reported in Table 2.

3.1.2.2. Other Noble Metals. Other noble metals tested in the decomposition of ammonia are rhodium and platinum. For example, using $\mathrm{Rh} / \mathrm{SiO}_{2}$ promoted with $\mathrm{Nb}$, Maeda et al. ${ }^{239}$ verified a superior catalytic activity to that of a catalyst composed of $\mathrm{Rh}$ supported on $\mathrm{Nb}_{2} \mathrm{O}_{5}$. Platinum supported on alumina was tested by Richardson et al., ${ }^{240}$ obtaining low catalytic activity. Catalysts based on noble metals other than ruthenium used to decompose ammonia and their catalytic performances are reported in Table 3 .

3.1.3. Non-noble Metals. Among the non-noble metals used as catalysts for the decomposition of ammonia, nickel is the element most studied as it shows remarkable activity. Again, the type of support has an important effect on the catalytic performance. Testing $\mathrm{Fe}$ and Ni catalysts supported on activated 
Table 3. Catalysts Based on Noble Metals Other than Ruthenium Used to Decompose Ammonia and Their Catalytic Performances at 1 atm

\begin{tabular}{|c|c|c|c|c|c|c|c|c|c|c|c|c|}
\hline $\begin{array}{l}\text { active } \\
\text { phase }\end{array}$ & wt $\%$ & support & promoter & $\begin{array}{c}\text { WHSV } \\
\left(\mathrm{mL} \mathrm{g}^{-1} \mathrm{~h}^{-1}\right)\end{array}$ & $\begin{array}{c}\text { GHSV } \\
\left(\mathrm{h}^{-1}\right)\end{array}$ & $\begin{array}{c}\% \mathrm{NH}_{3} \text { inlet } \\
\text { flow }\end{array}$ & $\begin{array}{c}\mathrm{T} \\
\left({ }^{\circ} \mathrm{C}\right)\end{array}$ & $\begin{array}{c}\text { conv } \\
(\%)\end{array}$ & $\begin{array}{c}\mathrm{H}_{2} \text { rate } \\
\left(\mathrm{mmol} \mathrm{g}^{-1} \mathrm{~s}^{-1}\right)\end{array}$ & $\begin{array}{c}E_{\mathrm{a}} \\
\left(\mathrm{kJ} \mathrm{mol}{ }^{-1}\right)\end{array}$ & $\begin{array}{l}\text { TOF } \\
\left(\mathrm{s}^{-1}\right)\end{array}$ & ref \\
\hline Ir & 1 & $\mathrm{Al}_{2} \mathrm{O}_{3}$ & & & & 100 & 580 & & & & 0.79 & 155 \\
\hline Ir & 10 & $\mathrm{Al}_{2} \mathrm{O}_{3}$ & & 30000 & & 100 & 450 & & & 82 & 0.7 & 158 \\
\hline Ir & 10 & $\mathrm{SiO}_{2}$ & & 30000 & & 100 & 450 & 8 & 0.04 & 72 & 2.1 & 158 \\
\hline $\mathrm{Pd}$ & & & & & 20600 & 100 & 450 & 13 & & & & 241 \\
\hline $\mathrm{Pd}$ & 0.5 & $\mathrm{Al}_{2} \mathrm{O}_{3}$ & & & & 100 & 580 & & & & 0.02 & 155 \\
\hline $\mathrm{Pd}$ & & CNTs & & 30000 & & 100 & 500 & 5 & 0.02 & 100 & & 156 \\
\hline $\mathrm{Pd}$ & 1 & $\mathrm{SiO}_{2}$ & & & 20600 & 100 & 450 & 16 & & & & 241 \\
\hline $\mathrm{Pt}$ & 1 & $\mathrm{Al}_{2} \mathrm{O}_{3}$ & & & & 100 & 580 & & & & 0.02 & 155 \\
\hline $\mathrm{Pt}$ & 1.7 & $\mathrm{Al}_{2} \mathrm{O}_{3}$ & & & & & 700 & 80 & & & & 240 \\
\hline $\mathrm{Pt}$ & 0.5 & C & & & 7500 & 100 & 570 & & & 100 & 0.07 & 242 \\
\hline $\mathrm{Pt}$ & & CNTs & & 30000 & & 100 & 500 & 4 & 0.02 & 88 & & 156 \\
\hline $\mathrm{Pt}$ & & $\begin{array}{l}\text { MCM- } \\
41\end{array}$ & & 5100 & & 100 & 500 & & 0.124 & & & 243 \\
\hline Pt & 10 & $\mathrm{SiO}_{2}$ & & 5100 & & 100 & 500 & & 0.084 & & & 243 \\
\hline $\mathrm{Rh}$ & 0.5 & $\mathrm{Al}_{2} \mathrm{O}_{3}$ & & & & 100 & 580 & & & & 2.26 & 155 \\
\hline $\mathrm{Rh}$ & & CNTs & & 30000 & & 100 & 500 & 15 & 0.07 & 81 & & 156 \\
\hline $\mathrm{Rh}$ & 5 & $\mathrm{Nb}_{2} \mathrm{O}_{5}$ & & & & 2.5 & & & & & & 239 \\
\hline $\mathrm{Rh}$ & 0.5 & $\mathrm{SiO}_{2}$ & $\mathrm{Nb}_{2} \mathrm{O}_{5}$ & & & 2.5 & & & & & & 239 \\
\hline
\end{tabular}

carbon, Donald et al. ${ }^{244}$ obtained a conversion with the $\mathrm{Fe}$ catalyst significantly higher with respect to the $\mathrm{Ni}$ catalyst. However, both catalysts showed good medium-term stability, demonstrating a progressive activation in $10 \mathrm{~h}$ of reaction. Comparing the catalytic activity of the transition metals $\mathrm{Ni}, \mathrm{Fe}$, and Co dispersed in an alumina matrix, $\mathrm{Gu}$ et al. ${ }^{245}$ obtained a higher catalytic activity with the cobalt catalyst, followed by the $\mathrm{Ni}$ catalyst and finally the lowest catalytic activity was obtained with $\mathrm{Fe} / \mathrm{Al}_{2} \mathrm{O}_{3}$. All three catalysts showed high stability for the decomposition reaction after more than $70 \mathrm{~h}$. The same order of catalytic activity was obtained with the catalysts supported on $\mathrm{MgO}$ modified with $\mathrm{La}^{246}$ and on CNTs. ${ }^{247}$ Similarly, Yanran et al. ${ }^{248}$ compared the decomposition of ammonia in the presence of $\mathrm{Fe}$ and Co catalysts and concluded that the latter has a higher activity ( 85 vs $30 \%$ conversion at $500{ }^{\circ} \mathrm{C}$ ). Conversely, both catalysts showed a decrease in activity after a second reaction cycle, although the addition of lanthanum to the catalyst resulted in a better stability for both catalysts. Comparing the catalytic results of different types of low-ordered carbon with and without adding $\mathrm{Fe}$ or $\mathrm{Ca}$, $\mathrm{Xu}$ et al. ${ }^{249}$ showed that an increase in conversion can be obtained with the catalyst promoted with $\mathrm{Fe}$, while adding Ca has a detrimental effect on the reaction.

3.1.3.1. Nickel. As mentioned above, the commercially available catalysts used for the industrial ammonia crackers are primarily composed of nickel supported on alumina. Catalysts based on nickel as active phase that are used to decompose ammonia and their catalytic performances are reported in Table 4. The work presented by Zhang et al. ${ }^{251}$ reported that the best TOF with $\mathrm{Ni} / \mathrm{Al}_{2} \mathrm{O}_{3}$ catalysts is obtained with a particle size of metallic Ni between 1.8 and $2.9 \mathrm{~nm}$, and that doping alumina with lanthanum increases the catalytic activity. In terms of $\mathrm{H}_{2}$ production, they obtained comparable results with $\mathrm{Ru}$ catalysts. ${ }^{252}$ Nickel has shown to have good synergy with rare earths, in particular La and Ce, used as promoters or in the form of oxides as support. In this sense, Yan et al. ${ }^{253}$ prepared a catalyst composed of $\mathrm{Ni}, \mathrm{Ni}_{0.5} \mathrm{Ce}_{0.5} \mathrm{O}_{x}, \mathrm{Ni}_{0.5} \mathrm{Al}_{0.5} \mathrm{O}_{x}$, or $\mathrm{Ni}_{0.5} \mathrm{Ce}_{0.1} \mathrm{Al}_{0.4} \mathrm{O}_{x}$ porous microspheres, and concluded that the catalyst composed of nickel and cerium gave better results compared to $\mathrm{Ni}_{0.5} \mathrm{Al}_{0.5} \mathrm{O}_{x}$, while $\mathrm{Ni}_{0.5} \mathrm{Ce}_{0.1} \mathrm{Al}_{0.4} \mathrm{O}_{x}$ allowed the stability of the catalyst to increase. Okura et al. ${ }^{254}$ tested different rare earth $(\mathrm{Y}, \mathrm{La}, \mathrm{Ce}, \mathrm{Sm}, \mathrm{Gd})$ and $\mathrm{Al}$ oxides as supports for $\mathrm{Ni}$, obtaining the highest conversion with $\mathrm{Y}_{2} \mathrm{O}_{3}$. Similar results were obtained by Nakamura and Fujitani ${ }^{255}$ comparing the supports $\mathrm{Y}_{2} \mathrm{O}_{3}, \mathrm{CeO}_{2}, \mathrm{MgO}, \mathrm{La}_{2} \mathrm{O}_{3}, \mathrm{Al}_{2} \mathrm{O}_{3}$, and $\mathrm{ZrO}_{2}$. However, the highest TOF value was obtained with the $\mathrm{ZrO}_{2}$ support. In a similar study carried out by Muroyama et al., ${ }^{256}$ the catalytic activity of supported $\mathrm{Ni}$ followed the trend: $\mathrm{Al}_{2} \mathrm{O}_{3} \approx \mathrm{La}_{2} \mathrm{O}_{3}>$ $\mathrm{SiO}_{2}>\mathrm{MgO} \approx \mathrm{CeO}_{2}>\mathrm{TiO}_{2}>\mathrm{ZrO}_{2}$.

To improve the catalytic performance of $\mathrm{Ni}$, rare earth promoters appear to have a positive effect. On the $\mathrm{Ni} / \mathrm{Al}_{2} \mathrm{O}_{3}$ catalyst, Okura et al. ${ }^{257}$ found that the promoting effect followed the trend $\mathrm{La} \approx \mathrm{Pr} \approx \mathrm{Nd}>\mathrm{Y}>\mathrm{Sm}>\mathrm{Eu} \approx \mathrm{Gd}>\mathrm{Ce}$. They also investigated the catalytic activity of oxides or mixtures of oxides as a support for $\mathrm{Ni}$, obtaining a trend of the catalytic activity that correlates well with the basicity of the materials: ${ }^{258} \mathrm{BaZrO}_{3}>$ $\mathrm{SrZrO}_{3}>\mathrm{SmAlO}_{3} \approx \mathrm{GdAlO}_{3}>\mathrm{SrTiO}_{3}>\mathrm{LaAlO}_{3} \approx \mathrm{BaTiO}_{3}>$ $\mathrm{Al}_{2} \mathrm{O}_{3}>\mathrm{CaMnO}_{3}>\mathrm{CaZrO}_{3}>\mathrm{SrMnO}_{3}>\mathrm{BaMnO}_{3}>\mathrm{MnO}_{2}>$ $\mathrm{NaNbO}_{3}>\mathrm{CaTiO}_{3} \approx \mathrm{KNbO}_{3}>\mathrm{Nb}_{2} \mathrm{O}_{5}>\mathrm{TiO}_{2}>\mathrm{ZrO}_{2}$. The catalysts supported on oxide mixtures showed superior catalytic activity compared to those prepared using simple oxides. In a similar way, Liu et al. ${ }^{259}$ observed that the conversion of ammonia increases when $\mathrm{Ni}$ microfibers are modified with $\mathrm{Al}_{2} \mathrm{O}_{3}$ and $\mathrm{CeO}_{2}$. Similar catalyst compositions were tested by Zheng et al., ${ }^{260}$ who doped Ni catalysts supported on alumina with ceria. The results showed that the addition of $\mathrm{CeO}_{2}$ improves the activity and the stability of the catalyst, and it is believed that ceria increases the dispersion of $\mathrm{Ni}$, improves its reducibility, and decreases metal sintering. Vacharapong et al. ${ }^{261}$ studied the same composition, but Ce was added to the support, and Liu et al. ${ }^{262}$ verified the promoting effect of $\mathrm{Ce}$ and La oxides on a Ni catalyst supported on SBA-15, obtaining an increase in conversion with $\mathrm{CeO}_{2}$ of around $10 \%$ at $500{ }^{\circ} \mathrm{C}$, and a limited increase using $\mathrm{La}_{2} \mathrm{O}_{3}(2 \%)$. In all these cases the ammonia conversion improved significantly as a result of the addition of $\mathrm{CeO}_{2}$ to the catalyst composition. Ceria is a well-known catalyst or catalyst component, and it is used in many applications due to its redox properties and ability to disperse and prevent sintering of metal nanoparticles through robust metal-support interactions. $^{263-266}$ 
Table 4. Catalysts Based on Nickel as Active Phase Used to Decompose Ammonia and Their Catalytic Performances at 1 atm

\begin{tabular}{|c|c|c|c|c|c|c|c|c|c|c|c|c|}
\hline active phase & wt $\%$ & support & promoter & $\begin{array}{c}\text { WHSV } \\
\left(\mathrm{mL} \mathrm{g}^{-1} \mathrm{~h}^{-1}\right)\end{array}$ & $\begin{array}{c}\text { GHSV } \\
\left(\mathrm{h}^{-1}\right)\end{array}$ & $\begin{array}{c}\% \\
\mathrm{NH}_{3} \\
\text { inlet } \\
\text { flow }\end{array}$ & $T\left({ }^{\circ} \mathrm{C}\right)$ & $\begin{array}{c}\text { conv } \\
(\%)\end{array}$ & $\begin{array}{c}\mathrm{H}_{2} \text { rate } \\
\left(\mathrm{mmol} \mathrm{g}^{-1} \mathrm{~s}^{-1}\right)\end{array}$ & $\begin{array}{c}E_{\mathrm{a}} \\
\left(\mathrm{kJ} \mathrm{mol}^{-1}\right)\end{array}$ & $\begin{array}{l}\text { TOF } \\
\left(s^{-1}\right)\end{array}$ & ref \\
\hline $\mathrm{Ni}$ & & & & 30000 & & 100 & 500 & 10 & 0.08 & & & 161 \\
\hline $\mathrm{Ni}_{0.5} \mathrm{Ce}_{0.1} \mathrm{Al}_{0.4} \mathrm{O}_{x}$ & & & & 18000 & & 100 & 500 & 88 & & 118 & & 253 \\
\hline $\mathrm{Ni}_{0.5} \mathrm{Ce}_{0.1} \mathrm{O}_{x}$ & & & & 18000 & & 100 & 500 & 81 & & 137 & & 253 \\
\hline $\mathrm{Ni}_{0.5} \mathrm{Al}_{0.5} \mathrm{O}_{x}$ & & & & 18000 & & 100 & 500 & 48 & & 114 & & 253 \\
\hline $\mathrm{Ni}$ & & & & & 12000 & 100 & 500 & 1 & & & 0.27 & 255 \\
\hline $\mathrm{Ni}$ & & & & & 10000 & 100 & 600 & 39 & & 115 & 1.20 & 259 \\
\hline $\mathrm{Ni}$ & & & & & 20600 & 100 & 450 & 12 & & & & 241 \\
\hline $\mathrm{Ni}$ & & & $\mathrm{Al}_{2} \mathrm{O}_{3}$ & & 10000 & 100 & 600 & 63 & & 109 & 0.21 & 259 \\
\hline $\mathrm{Ni}$ & & & $\mathrm{CeO}_{2}$ & & 10000 & 100 & 600 & 65 & & 113 & 0.51 & 259 \\
\hline $\mathrm{Ni}$ & & & $\begin{array}{c}\mathrm{CeO}_{2} / \\
\mathrm{Al}_{2} \mathrm{O}_{3}\end{array}$ & & 10000 & 100 & 600 & 81 & & 105 & 0.30 & 259 \\
\hline $\mathrm{Ni}$ & & $@ \mathrm{Al}_{2} \mathrm{O}_{3}$ & & 30000 & & 100 & 450 & 9 & & & & 162 \\
\hline $\mathrm{Ni}$ & & $@ \mathrm{MgO}$ & & 30000 & & 100 & 450 & 10 & & & & 162 \\
\hline $\mathrm{Ni}$ & & $@ \mathrm{SiO}_{2}$ & & 30000 & & 100 & 500 & 36 & 0.20 & 63 & 6.5 & 161 \\
\hline $\mathrm{Ni}$ & & $@ \mathrm{SiO}_{2}$ & $\mathrm{Ce}$ & 30000 & & 100 & 500 & 40 & & & & 161 \\
\hline $\mathrm{Ni}$ & & $@ \mathrm{SiO}_{2}$ & $\mathrm{Ce}$ & & & 100 & 600 & 87 & 0.49 & 57 & & 271 \\
\hline $\mathrm{Ni}$ & & $@ \mathrm{SiO}_{2}$ & $\mathrm{La}$ & 30000 & & 100 & 500 & 47 & 0.26 & & & 161 \\
\hline $\mathrm{Ni}$ & 5 & $\mathrm{AC}$ & & & 6000 & 100 & 500 & 24 & & & 1.9 & 284 \\
\hline $\mathrm{Ni}$ & 10 & $\mathrm{AC}$ & & 30000 & & 100 & 500 & 2 & & & & 285 \\
\hline $\mathrm{Ni}$ & 13 & $\mathrm{AC}$ & & & 45000 & 0.2 & 750 & 75 & & & & 244 \\
\hline $\mathrm{Ni}$ & & $\mathrm{Al}_{2} \mathrm{O}_{3}$ & & 6000000 & & 15 & 540 & & & 96 & 30 & 251 \\
\hline $\mathrm{Ni}$ & & $\mathrm{Al}_{2} \mathrm{O}_{3}$ & & 30000 & & 100 & 450 & 15 & 0.08 & & & 252 \\
\hline $\mathrm{Ni}$ & & $\mathrm{Al}_{2} \mathrm{O}_{3}$ & & 30000 & & 100 & 450 & 15 & & & & 260 \\
\hline $\mathrm{Ni}$ & & $\mathrm{Al}_{2} \mathrm{O}_{3}$ & & 60000 & & 100 & 600 & 29 & & & & 288 \\
\hline $\mathrm{Ni}$ & & $\mathrm{Al}_{2} \mathrm{O}_{3}$ & & 60000 & & 100 & 600 & 17 & & & & 289 \\
\hline $\mathrm{Ni}$ & & $\mathrm{Al}_{2} \mathrm{O}_{3}$ & & & 1800 & 100 & 650 & 97 & & & & 290 \\
\hline $\mathrm{Ni}$ & 1 & $\mathrm{Al}_{2} \mathrm{O}_{3}$ & & & & 100 & 580 & & & & 4.21 & 155 \\
\hline $\mathrm{Ni}$ & 1.7 & $\mathrm{Al}_{2} \mathrm{O}_{3}$ & & & & 100 & 500 & 30 & & & & 282 \\
\hline $\mathrm{Ni}$ & 3.6 & $\mathrm{Al}_{2} \mathrm{O}_{3}$ & & & & 100 & 550 & 33 & & 100 & & 278 \\
\hline $\mathrm{Ni}$ & 8.9 & $\mathrm{Al}_{2} \mathrm{O}_{3}$ & & & 9000 & 100 & 500 & 27 & & 92 & 0.39 & 279 \\
\hline $\mathrm{Ni}$ & 10 & $\mathrm{Al}_{2} \mathrm{O}_{3}$ & & 6000 & & 100 & 450 & 10 & & & & 254 \\
\hline $\mathrm{Ni}$ & 10 & $\mathrm{Al}_{2} \mathrm{O}_{3}$ & & 6000 & & 100 & 500 & 35 & & & & 256 \\
\hline $\mathrm{Ni}$ & 10 & $\mathrm{Al}_{2} \mathrm{O}_{3}$ & & 6000 & & 100 & 450 & 10 & & & & 257 \\
\hline $\mathrm{Ni}$ & 10 & $\mathrm{Al}_{2} \mathrm{O}_{3}$ & & 240000 & 80000 & 50 & 500 & 93 & & & & 218 \\
\hline $\mathrm{Ni}$ & 10 & $\mathrm{Al}_{2} \mathrm{O}_{3}$ & & 9000 & & 5.9 & 400 & 32 & & & & 291 \\
\hline $\mathrm{Ni}$ & 10 & $\mathrm{Al}_{2} \mathrm{O}_{3}$ & & 13800 & 3067 & 57 & 400 & 8 & & & 0.01 & 219 \\
\hline $\mathrm{Ni}$ & 20 & $\mathrm{Al}_{2} \mathrm{O}_{3}$ & & 7500 & & 100 & 500 & 28 & & 84 & & 261 \\
\hline $\mathrm{Ni}$ & 20 & $\mathrm{Al}_{2} \mathrm{O}_{3}$ & & 2400 & & 100 & 500 & 31 & 0.007 & & & 275 \\
\hline $\mathrm{Ni}$ & 20 & $\mathrm{Al}_{2} \mathrm{O}_{3}$ & & 7500 & & 100 & 500 & 27 & 0.03 & 88 & & 276 \\
\hline $\mathrm{Ni}$ & 30 & $\mathrm{Al}_{2} \mathrm{O}_{3}$ & & & 12000 & 100 & 500 & 42 & & & 0.39 & 255 \\
\hline $\mathrm{Ni}$ & 40 & $\mathrm{Al}_{2} \mathrm{O}_{3}$ & & 6000 & & 100 & 550 & 66 & & & & 258 \\
\hline $\mathrm{Ni}$ & 71.4 & $\mathrm{Al}_{2} \mathrm{O}_{3}$ & & 30000 & & 100 & 550 & 51 & & & 0.4 & 223 \\
\hline $\mathrm{Ni}$ & 90 & $\mathrm{Al}_{2} \mathrm{O}_{3}$ & & 36000 & & 100 & 600 & 93 & & 123 & & 245 \\
\hline $\mathrm{Ni}$ & & $\mathrm{Al}_{2} \mathrm{O}_{3}$ & $\mathrm{Ce}$ & 30000 & & 100 & 450 & 28 & & & & 260 \\
\hline $\mathrm{Ni}$ & 9 & $\mathrm{Al}_{2} \mathrm{O}_{3}$ & $\mathrm{Ce}$ & 6000 & & 100 & 450 & 15 & & & & 257 \\
\hline $\mathrm{Ni}$ & 20 & $\mathrm{Al}_{2} \mathrm{O}_{3}$ & $\mathrm{Ce}$ & 7500 & & 100 & 500 & 51 & & 83 & & 261 \\
\hline $\mathrm{Ni}$ & 9 & $\mathrm{Al}_{2} \mathrm{O}_{3}$ & $\mathrm{Eu}$ & 6000 & & 100 & 450 & 16 & & & & 257 \\
\hline $\mathrm{Ni}$ & 9 & $\mathrm{Al}_{2} \mathrm{O}_{3}$ & Gd & 6000 & & 100 & 450 & 16 & & & & 257 \\
\hline $\mathrm{Ni}$ & 9 & $\mathrm{Al}_{2} \mathrm{O}_{3}$ & $\mathrm{La}$ & 6000 & & 100 & 450 & 20 & & & & 257 \\
\hline $\mathrm{Ni}$ & 9 & $\mathrm{Al}_{2} \mathrm{O}_{3}$ & $\mathrm{Nd}$ & 6000 & & 100 & 450 & 20 & & & & 257 \\
\hline $\mathrm{Ni}$ & 9 & $\mathrm{Al}_{2} \mathrm{O}_{3}$ & $\mathrm{Pr}$ & 6000 & & 100 & 450 & 20 & & & & 257 \\
\hline $\mathrm{Ni}$ & 9 & $\mathrm{Al}_{2} \mathrm{O}_{3}$ & $\mathrm{Sm}$ & 6000 & & 100 & 450 & 18 & & & & 257 \\
\hline $\mathrm{Ni}$ & 9 & $\mathrm{Al}_{2} \mathrm{O}_{3}$ & $\mathrm{Y}$ & 6000 & & 100 & 450 & 19 & & & & 257 \\
\hline $\mathrm{Ni}$ & 20 & $\mathrm{Al}_{2} \mathrm{O}_{3}$ & $\mathrm{Zr}$ & 7500 & & 100 & 500 & 29 & 0.04 & & & 276 \\
\hline $\mathrm{Ni}$ & 8 & $\mathrm{Al}-\mathrm{Ce}_{0.8} \mathrm{Zr}_{0.2} \mathrm{O}_{2}$ & & & 9000 & 100 & 500 & 58 & & 67 & 0.48 & 279 \\
\hline $\mathrm{Ni}$ & 38.6 & attapulgite & & 30000 & & 100 & 650 & 90 & 0.50 & 75 & & 273 \\
\hline $\mathrm{Ni}$ & 8.7 & attapulgite@SiO 2 & & 30000 & & 100 & 650 & 73 & 0.41 & 80 & & 273 \\
\hline $\mathrm{Ni}$ & 40 & $\mathrm{BaMnO}_{3}$ & & 6000 & & 100 & 550 & 46 & & & & 258 \\
\hline $\mathrm{Ni}$ & 40 & $\mathrm{BaTiO}_{3}$ & & 6000 & & 100 & 550 & 75 & & & & 258 \\
\hline
\end{tabular}


Table 4. continued

\begin{tabular}{|c|c|c|c|c|c|c|c|c|c|c|c|c|}
\hline active phase & wt $\%$ & support & promoter & $\begin{array}{c}\text { WHSV } \\
\left(\mathrm{mL} \mathrm{g}^{-1} \mathrm{~h}^{-1}\right)\end{array}$ & $\begin{array}{l}\text { GHSV } \\
\left(\mathrm{h}^{-1}\right)\end{array}$ & $\begin{array}{c}\% \\
\mathrm{NH}_{3} \\
\text { inlet } \\
\text { flow }\end{array}$ & $T\left({ }^{\circ} \mathrm{C}\right)$ & $\begin{array}{l}\text { conv } \\
(\%)\end{array}$ & $\begin{array}{c}\mathrm{H}_{2} \text { rate } \\
\left(\mathrm{mmol} \mathrm{g}^{-1} \mathrm{~s}^{-1}\right)\end{array}$ & $\begin{array}{c}E_{\mathrm{a}} \\
\left(\mathrm{kJ} \mathrm{mol}^{-1}\right)\end{array}$ & $\begin{array}{l}\text { TOF } \\
\left(s^{-1}\right)\end{array}$ & ref \\
\hline $\mathrm{Ni}$ & 40 & $\mathrm{BaZrO}_{3}$ & & 6000 & & 100 & 550 & 94 & & & & 258 \\
\hline $\mathrm{Ni}$ & 5 & $\mathrm{C}$ & & & 13000 & 10 & 400 & 19 & & & & 224 \\
\hline $\mathrm{Ni}$ & 40 & $\mathrm{CaMnO}_{3}$ & & 6000 & & 100 & 550 & 54 & & & & 258 \\
\hline $\mathrm{Ni}$ & 40 & $\mathrm{CaTiO}_{3}$ & & 6000 & & 100 & 550 & 36 & & & & 258 \\
\hline $\mathrm{Ni}$ & 40 & $\mathrm{CaZrO}_{3}$ & & 6000 & & 100 & 550 & 51 & & & & 258 \\
\hline $\mathrm{Ni}$ & 10 & $\mathrm{Ce}_{0.6} \mathrm{Zr}_{0.3} \mathrm{Y}_{0.1} \mathrm{O}_{2}$ & & 6000 & & 100 & 350 & 7 & 0.007 & 47 & 0.52 & 292 \\
\hline $\mathrm{Ni}$ & 10.7 & $\mathrm{Ce}_{0.8} \mathrm{Zr}_{0.2} \mathrm{O}_{2}$ & & & 9000 & 100 & 500 & 48 & & 74 & 0.34 & 279 \\
\hline $\mathrm{Ni}$ & 13.2 & $\mathrm{Ce}_{0.8} \mathrm{Zr}_{0.2} \mathrm{O}_{2}$ & & & & 100 & 550 & 96 & & 54 & & 278 \\
\hline $\mathrm{Ni}$ & 20 & $\mathrm{Ce}-\mathrm{Al}_{2} \mathrm{O}_{3}$ & & 7500 & & 100 & 500 & 53 & & 41 & & 277 \\
\hline $\mathrm{Ni}$ & & $\mathrm{CeO}_{2}$ & & & & 100 & 600 & 77 & 0.43 & 70 & & 271 \\
\hline $\mathrm{Ni}$ & 3.6 & $\mathrm{CeO}_{2}$ & & & & 100 & 550 & 61 & & 71 & & 278 \\
\hline $\mathrm{Ni}$ & 10 & $\mathrm{CeO}_{2}$ & & 6000 & & 100 & 450 & 3 & & & & 254 \\
\hline $\mathrm{Ni}$ & 10 & $\mathrm{CeO}_{2}$ & & 6000 & & 100 & 500 & 20 & & & & 256 \\
\hline $\mathrm{Ni}$ & 10 & $\mathrm{CeO}_{2}$ & & 6000 & & 100 & 450 & 30 & & & & 292 \\
\hline $\mathrm{Ni}$ & 10 & $\mathrm{CeO}_{2}$ & & 13800 & 3067 & 57 & 400 & 13 & & & 0.03 & 219 \\
\hline $\mathrm{Ni}$ & 30 & $\mathrm{CeO}_{2}$ & & & 12000 & 100 & 500 & 60 & & & 0.66 & 255 \\
\hline $\mathrm{Ni}$ & & CNTs & & 30000 & & 100 & 500 & 9 & 0.05 & 90 & & 156 \\
\hline $\mathrm{Ni}$ & 5 & CNTs & & 6000 & & 100 & 500 & 25 & & 121 & & 247 \\
\hline $\mathrm{Ni}$ & 5 & CNTs & & & & & & & & & & 233 \\
\hline $\mathrm{Ni}$ & 5 & fumed $\mathrm{SiO}_{2}$ & & 30000 & & 100 & 500 & 7 & & & & 164 \\
\hline $\mathrm{Ni}$ & 5 & fumed $\mathrm{SiO}_{2}$ & $\mathrm{~K}$ & 30000 & & 100 & 500 & 8 & & & & 164 \\
\hline $\mathrm{Ni}$ & 10 & $\mathrm{Gd}_{2} \mathrm{O}_{3}$ & & 6000 & & 100 & 450 & 16 & & & & 254 \\
\hline $\mathrm{Ni}$ & 40 & $\mathrm{GdAlO}_{3}$ & & 6000 & & 100 & 550 & 81 & & & & 258 \\
\hline $\mathrm{Ni}$ & 10 & GNP & & 15000 & 12000 & 10 & 375 & 17 & & 86 & & 235 \\
\hline $\mathrm{Ni}$ & 10 & GO & & 30000 & & 100 & 500 & 17 & & 65 & & 285 \\
\hline $\mathrm{Ni}$ & 5 & graphene & & & 6000 & 100 & 500 & $<1$ & & & & 283 \\
\hline $\mathrm{Ni}$ & 10 & $\mathrm{HY}$ & & 30000 & & 100 & 450 & & & & 0.8 & 158 \\
\hline $\mathrm{Ni}$ & 23.6 & hydrocalumite & & 10000 & & 100 & 500 & 55 & & & & 280 \\
\hline $\mathrm{Ni}$ & 10 & HZSM-5 & & 30000 & & 100 & 450 & & & 84 & 0.1 & 158 \\
\hline $\mathrm{Ni}$ & 40 & $\mathrm{KNbO}_{3}$ & & 6000 & & 100 & 550 & 36 & & & & 258 \\
\hline $\mathrm{Ni}$ & 10 & $\mathrm{La}_{2} \mathrm{O}_{3}$ & & 6000 & & 100 & 450 & 12 & & & & 254 \\
\hline $\mathrm{Ni}$ & 10 & $\mathrm{La}_{2} \mathrm{O}_{3}$ & & 6000 & & 100 & 500 & 34 & & & & 256 \\
\hline $\mathrm{Ni}$ & 30 & $\mathrm{La}_{2} \mathrm{O}_{3}$ & & & 12000 & 100 & 500 & 49 & & & 0.61 & 255 \\
\hline $\mathrm{Ni}$ & & $\mathrm{La}-\mathrm{Al}_{2} \mathrm{O}_{3}$ & & 6000000 & & 15 & 540 & & & 98 & 6 & 251 \\
\hline $\mathrm{Ni}$ & & $\mathrm{La}-\mathrm{Al}_{2} \mathrm{O}_{3}$ & & 30000 & & 100 & 450 & 26 & 0.15 & & & 252 \\
\hline $\mathrm{Ni}$ & 40 & $\mathrm{LaAlO}_{3}$ & & 6000 & & 100 & 550 & 76 & & & & 258 \\
\hline $\mathrm{Ni}$ & 20 & $\mathrm{La}-\mathrm{MgO}$ & & 22000 & & 100 & 400 & 28 & & 182 & $0.007^{a}$ & 246 \\
\hline $\mathrm{Ni}$ & & MCF-17 & & 6000 & & 100 & 500 & 40 & & & & 269 \\
\hline $\mathrm{Ni}$ & 5 & MCM-41 & & 30000 & & 100 & 500 & 9 & & & & 164 \\
\hline $\mathrm{Ni}$ & 7 & MCM-41 & & 36000 & & 100 & 500 & 58 & & & 0.62 & 270 \\
\hline $\mathrm{Ni}$ & 5 & MCM-41 & $\mathrm{K}$ & 30000 & & 100 & 500 & 11 & & 49 & 1.7 & 164 \\
\hline $\mathrm{Ni}$ & & $\mathrm{MgO}$ & & 3000 & & 100 & 500 & 56 & & & 0.51 & 281 \\
\hline $\mathrm{Ni}$ & 6 & $\mathrm{MgO}$ & & & 1800 & 100 & 650 & 88 & & & & 290 \\
\hline $\mathrm{Ni}$ & 10 & $\mathrm{MgO}$ & & 6000 & & 100 & 500 & 22 & & & & 256 \\
\hline $\mathrm{Ni}$ & 30 & $\mathrm{MgO}$ & & & 15000 & 100 & 450 & 12 & & 144 & & 238 \\
\hline $\mathrm{Ni}$ & 30 & $\mathrm{MgO}$ & & & 12000 & 100 & 500 & 51 & & & 0.46 & 255 \\
\hline $\mathrm{Ni}$ & 15 & $\mathrm{MgO}-\mathrm{Al}_{2} \mathrm{O}_{3}$ & & 3000 & & 100 & 500 & 59 & & & 0.20 & 281 \\
\hline $\mathrm{Ni}$ & 41.8 & $\mathrm{Mg}_{y} \mathrm{Al}_{z} \mathrm{O}_{\mathrm{n}}$ & & 30000 & & 100 & 550 & 79 & & & 0.7 & 223 \\
\hline $\mathrm{Ni}$ & 40 & $\mathrm{MnO}_{2}$ & & 6000 & & 100 & 550 & 43 & & & & 258 \\
\hline $\mathrm{Ni}$ & 5 & MWCNTs & & & 6000 & 100 & 500 & 25 & & & & 283 \\
\hline $\mathrm{Ni}$ & 5 & MWCNTs & & & 6000 & 100 & 500 & 57 & & & 1.3 & 284 \\
\hline $\mathrm{Ni}$ & 40 & $\mathrm{NaNbO}_{3}$ & & 6000 & & 100 & 550 & 39 & & & & 258 \\
\hline $\mathrm{Ni}$ & 40 & $\mathrm{Nb}_{2} \mathrm{O}_{5}$ & & 6000 & & 100 & 550 & 34 & & & & 258 \\
\hline $\mathrm{Ni}$ & 10 & OMC & & 30000 & & 100 & 500 & 8 & & & & 285 \\
\hline $\mathrm{Ni}$ & 15 & red mud & & 30000 & & 100 & 500 & 16 & & 70 & & 286 \\
\hline $\mathrm{Ni}$ & & SBA-15 & & 30000 & & 100 & 500 & 57 & & & & 262 \\
\hline $\mathrm{Ni}$ & 5 & SBA-15 & & 30000 & & 100 & 500 & 7 & & & & 164 \\
\hline $\mathrm{Ni}$ & 23.4 & SBA-15 & & 30000 & & 100 & 550 & 89 & 0.50 & & & 272 \\
\hline
\end{tabular}


Table 4. continued

\begin{tabular}{|c|c|c|c|c|c|c|c|c|c|c|c|c|}
\hline active phase & wt $\%$ & support & promoter & $\begin{array}{c}\text { WHSV } \\
\left(\mathrm{mL} \mathrm{g}^{-1} \mathrm{~h}^{-1}\right)\end{array}$ & $\begin{array}{c}\text { GHSV } \\
\left(\mathrm{h}^{-1}\right)\end{array}$ & $\begin{array}{c}\% \\
\mathrm{NH}_{3} \\
\text { inlet } \\
\text { flow }\end{array}$ & $T\left({ }^{\circ} \mathrm{C}\right)$ & $\begin{array}{c}\text { conv } \\
(\%)\end{array}$ & $\begin{array}{c}\mathrm{H}_{2} \text { rate } \\
\left(\mathrm{mmol} \mathrm{g}^{-1} \mathrm{~s}^{-1}\right)\end{array}$ & $\begin{array}{c}E_{\mathrm{a}} \\
\left(\mathrm{kJ} \mathrm{mol}{ }^{-1}\right)\end{array}$ & $\begin{array}{l}\text { TOF } \\
\left(\mathrm{s}^{-1}\right)\end{array}$ & ref \\
\hline $\mathrm{Ni}$ & & SBA-15 & $\mathrm{Ce}$ & 30000 & & 100 & 500 & 66 & & & & 262 \\
\hline $\mathrm{Ni}$ & 5 & SBA-15 & $\mathrm{K}$ & 30000 & & 100 & 500 & 8 & & & & 164 \\
\hline $\mathrm{Ni}$ & & SBA-15 & $\mathrm{La}$ & 30000 & & 100 & 500 & 59 & & & & 262 \\
\hline $\mathrm{Ni}$ & 5.2 & sepiolite & & 2000 & & 100 & 550 & 82 & 0.03 & 105 & & 274 \\
\hline $\mathrm{Ni}$ & & $\mathrm{SiO}_{2}$ & & & 20600 & 100 & 450 & 4 & & & & 241 \\
\hline $\mathrm{Ni}$ & 10 & $\mathrm{SiO}_{2}$ & & 30000 & & 100 & 450 & 4 & 0.02 & & 1.0 & 158 \\
\hline $\mathrm{Ni}$ & 10 & $\mathrm{SiO}_{2}$ & & 6000 & & 100 & 500 & 31 & & & & 256 \\
\hline $\mathrm{Ni}$ & 10 & $\mathrm{SiO}_{2}$ & & 36000 & 1200 & 100 & 550 & 50 & & 108 & & 268 \\
\hline $\mathrm{Ni}$ & 65 & $\mathrm{SiO}_{2}-\mathrm{Al}_{2} \mathrm{O}_{3}$ & & 30000 & & 100 & 450 & 9 & 0.05 & 92 & & 158 \\
\hline $\mathrm{Ni}$ & 66 & $\mathrm{SiO}_{2}-\mathrm{Al}_{2} \mathrm{O}_{3}$ & & 7200 & & 100 & 450 & 34 & & & 0.003 & 32 \\
\hline $\mathrm{Ni}$ & 10 & $\mathrm{Sm}_{2} \mathrm{O}_{3}$ & & 6000 & & 100 & 450 & 15 & & & & 254 \\
\hline $\mathrm{Ni}$ & 40 & $\mathrm{SmAlO}_{3}$ & & 6000 & & 100 & 550 & 81 & & & & 258 \\
\hline $\mathrm{Ni}$ & 20 & $\mathrm{Sr}-\mathrm{Al}_{2} \mathrm{O}_{3}$ & & 7500 & & 100 & 500 & 45 & & 43 & & 277 \\
\hline $\mathrm{Ni}$ & 40 & $\mathrm{SrMnO}_{3}$ & & 6000 & & 100 & 550 & 48 & & & & 258 \\
\hline $\mathrm{Ni}$ & 40 & $\mathrm{SrTiO}_{3}$ & & 6000 & & 100 & 550 & 80 & & & & 258 \\
\hline $\mathrm{Ni}$ & 40 & $\mathrm{SrZrO}_{3}$ & & 6000 & & 100 & 550 & 90 & & & & 258 \\
\hline $\mathrm{Ni}$ & 5 & SWCNTs & & & 6000 & 100 & 500 & $<1$ & & & & 283 \\
\hline $\mathrm{Ni}$ & 10 & $\mathrm{TiO}_{2}$ & & 6000 & & 100 & 500 & 16 & & & & 256 \\
\hline $\mathrm{Ni}$ & 40 & $\mathrm{TiO}_{2}$ & & 6000 & & 100 & 550 & 31 & & & & 258 \\
\hline $\mathrm{Ni}$ & 10 & $\mathrm{Y}_{2} \mathrm{O}_{3}$ & & 6000 & & 100 & 450 & 18 & & & 0.9 & 254 \\
\hline $\mathrm{Ni}$ & 10 & $\mathrm{Y}_{2} \mathrm{O}_{3}$ & & 6000 & & 100 & 450 & 23 & & & & 292 \\
\hline $\mathrm{Ni}$ & 30 & $\mathrm{Y}_{2} \mathrm{O}_{3}$ & & & 12000 & 100 & 500 & 61 & & & 0.42 & 255 \\
\hline $\mathrm{Ni}$ & 20 & $\mathrm{Y}-\mathrm{Al}_{2} \mathrm{O}_{3}$ & & 7500 & & 100 & 500 & 43 & & 43 & & 277 \\
\hline $\mathrm{Ni}$ & 1.6 & zeolite & & & & 100 & 500 & 61 & & 113 & & 282 \\
\hline $\mathrm{Ni}$ & 20 & $\mathrm{Zr}-\mathrm{Al}_{2} \mathrm{O}_{3}$ & & 7500 & & 100 & 500 & 49 & & 42 & & 277 \\
\hline $\mathrm{Ni}$ & 10 & $\mathrm{ZrO}_{2}$ & & 6000 & & 100 & 500 & 4 & & & & 256 \\
\hline $\mathrm{Ni}$ & 10 & $\mathrm{ZrO}_{2}$ & & 6000 & & 100 & 450 & 13 & & & & 292 \\
\hline $\mathrm{Ni}$ & 30 & $\mathrm{ZrO}_{2}$ & & & 12000 & 100 & 500 & 31 & & & 0.81 & 255 \\
\hline $\mathrm{Ni}$ & 40 & $\mathrm{ZrO}_{2}$ & & 6000 & & 100 & 550 & 27 & & & & 258 \\
\hline $\mathrm{Ni}$ & 20 & $\mathrm{ZrO}_{2}-\mathrm{Al}_{2} \mathrm{O}_{3}$ & & 2400 & & 100 & 500 & 42 & 0.010 & & & 275 \\
\hline $\mathrm{Ni}$ & 20 & $\mathrm{ZrO}_{2}-\mathrm{Al}_{2} \mathrm{O}_{3}$ & & 7500 & & 100 & 500 & 43 & 0.05 & 86 & & 276 \\
\hline $\mathrm{Ni}$ & 5 & ZSM-5 & & 30000 & & 100 & 650 & 98 & 0.55 & 88 & & 293 \\
\hline
\end{tabular}

In the case of nickel catalysts, silica has also been tested as a support in various forms, such as $\mathrm{SiO}_{2}$, mesoporous structures, or as a natural mineral. Choudhary et al. ${ }^{158,267}$ tested various types of silica and zeolites ( $\mathrm{HY}$ and $\mathrm{H}-\mathrm{ZSM}-5$ ) as a support for $\mathrm{Ni}$, and also a catalyst supported on a mixture of silica and alumina, obtaining the order of catalytic activity: $\mathrm{SiO}_{2}-\mathrm{Al}_{2} \mathrm{O}_{3}>$ $\mathrm{SiO}_{2} \approx \mathrm{HY} \gg \mathrm{H}-\mathrm{ZSM}-5$. The relationship between the catalytic activity and porosity using a $\mathrm{Ni} / \mathrm{SiO}_{2}$ catalyst was investigated by Atsumi et al. ${ }^{268}$ It was found that the catalyst with the smallest pore diameter $(7.7 \mathrm{~nm})$ presented the highest activity at temperatures below $650{ }^{\circ} \mathrm{C}$, while at higher temperatures the conversion was higher with larger pores as a consequence of internal mass transfer issues. Good catalytic activities were also obtained using Ni supported on mesoporous silica MCF-1 $7^{269}$ and MCM-41. ${ }^{270}$ Similarly, Yao et al. ${ }^{161}$ showed that by encapsulating $\mathrm{Ni}$ in $\mathrm{SiO}_{2}$, the catalytic activity increased more than $25 \%$ at $500{ }^{\circ} \mathrm{C}$ compared to the noncapsulated catalyst. Moreover, by adding $\mathrm{Ce}$ or La to the catalyst, the activity increases by 11 and $4 \%$, respectively, compared to $\mathrm{Ni}$ encapsulated in $\mathrm{SiO}_{2}$ without promoters. The same $\mathrm{Ni}$ nanoparticles encapsulated in $\mathrm{Al}_{2} \mathrm{O}_{3}$ or $\mathrm{MgO}$ presented a lower catalytic activity, 9 and $10 \%$ conversion at $450{ }^{\circ} \mathrm{C}$, respectively, compared to a conversion of $17 \%$ for the catalyst encapsulated in $\mathrm{SiO}_{2} .{ }^{162} \mathrm{Zhang}$ et al. ${ }^{271}$ expanded the study of the $\mathrm{Ni}$ catalyst encapsulated in $\mathrm{SiO}_{2}$ by adding ceria as a promoter, obtaining a higher activity with respect to $\mathrm{Ni}$ supported on ceria. By using Ni catalyst supported on SBA-15 mesoporous silica, Liu et al. ${ }^{272}$ obtained a higher conversion of ammonia with respect to $\mathrm{Ru}$ catalysts supported on various types of carbon under the same reaction conditions. ${ }^{183}$ A nickel catalyst supported on attapulgite (a type of clay composed mostly of silica and $\mathrm{MgO}$ ) was tested and its activity was compared with the same catalyst supported on silica. At parity of $\mathrm{Ni}$ content, the $\mathrm{Ni} / \mathrm{SiO}_{2}$ catalyst showed superior activity and stability. ${ }^{273}$ In contrast, sepiolite (an abundant $\mathrm{Si}$ and $\mathrm{Mg}$ mineral) has demonstrated good catalytic results as a Ni support for the decomposition of ammonia. ${ }^{274}$

Other types of compounds tested as supports for $\mathrm{Ni}$ are zirconium-based supports. For instance, Henpraserttae et al. ${ }^{275}$ tested the use of $\mathrm{ZrO}_{2}$ as dopant for an alumina support, obtaining an increase in ammonia conversion of $11 \%$ at $500{ }^{\circ} \mathrm{C}$ compared to the catalyst supported only on alumina. The catalyst supported on the oxide mixture showed superior catalytic activity compared to the catalyst with the promoter added directly to the active Ni phase. ${ }^{276}$ The better activity of the $\mathrm{Zr}$-doped sample is attributed to a greater dispersion of $\mathrm{Ni}$, as well as an increase in basic sites. Doping alumina with other elements shows that the catalytic activity follows the order $\mathrm{Ce}>$ 
Table 5. Catalysts Based on Iron as Active Phase Used to Decompose Ammonia and Their Catalytic Performances at 1 atm

\begin{tabular}{|c|c|c|c|c|c|c|c|c|c|c|c|c|}
\hline active phase & wt $\%$ & support & promoter & $\begin{array}{c}\text { WHSV } \\
\left(\mathrm{mL} \mathrm{g}^{-1} \mathrm{~h}^{-1}\right)\end{array}$ & $\begin{array}{l}\text { GHSV } \\
\left(\mathrm{h}^{-1}\right)\end{array}$ & $\begin{array}{l}\% \mathrm{NH}_{3} \\
\text { inlet } \\
\text { flow }\end{array}$ & $T\left({ }^{\circ} \mathrm{C}\right)$ & $\begin{array}{c}\text { conv } \\
(\%)\end{array}$ & $\begin{array}{c}\mathrm{H}_{2} \text { rate } \\
\left(\mathrm{mmol} \mathrm{g}^{-1} \mathrm{~s}^{-1}\right)\end{array}$ & $\begin{array}{c}E_{\mathrm{a}} \\
\left(\mathrm{kJ} \mathrm{mol}{ }^{-1}\right)\end{array}$ & $\begin{array}{l}\text { TOF } \\
\left(\mathrm{s}^{-1}\right)\end{array}$ & ref \\
\hline $\mathrm{Fe}$ & & & & 30000 & & 100 & 500 & 9 & 0.05 & & & 160 \\
\hline $\mathrm{Fe}$ & & & & 18000 & & 100 & 500 & 30 & 0.002 & & & 248 \\
\hline $\mathrm{Fe}$ & & & & & & & 700 & 100 & & & & 297 \\
\hline $\mathrm{Fe}$ & & & & 15000 & & 100 & 550 & 4 & & & & 298 \\
\hline $\mathrm{Fe}$ & & & & 24000 & & 100 & 550 & 33 & & & & 308 \\
\hline Fe (Limonite) & & & & 45000 & & 0.2 & 450 & 88 & & 100 & & 309 \\
\hline $\mathrm{Fe}$ & & & & 60 & & 100 & 400 & 28 & & & & 312 \\
\hline $\begin{array}{l}\mathrm{Fe} \\
\quad\left(\mathrm{C}_{32} \mathrm{H}_{16} \mathrm{FeN}_{8}\right)\end{array}$ & & & & 60000 & & 100 & 600 & 33 & & & & 289 \\
\hline $\begin{array}{l}\mathrm{Fe} \\
\quad(\text { Amomax } 10)\end{array}$ & & & & 120000 & & 0.4 & 500 & 11 & & & & 122 \\
\hline $\mathrm{Fe}$ & & & & & & 15 & 550 & 4 & & & & 318 \\
\hline $\mathrm{Fe}$ & & & $\mathrm{Al}, \mathrm{K}$ & 1500 & & 0.6 & 500 & 66 & & & & 319 \\
\hline $\mathrm{Fe}$ (Magnetite) & & & $\mathrm{Al}_{2} \mathrm{O}_{3}, \mathrm{CaO}$ & 30000 & & 100 & 500 & 40 & & & & 296 \\
\hline $\mathrm{Fe}$ & & & $\mathrm{CaO}, \mathrm{Al}_{2} \mathrm{O}_{3}$ & & & 5 & 400 & & & 167 & 0.0110 & 295 \\
\hline $\mathrm{Fe}$ & & & $\mathrm{CaO}, \mathrm{Al}_{2} \mathrm{O}_{3}, \mathrm{~K}_{2} \mathrm{O}$ & & & 5 & 400 & & & 146 & 0.0105 & 295 \\
\hline $\mathrm{Fe}$ & & & $\mathrm{CaO}, \mathrm{Al}_{2} \mathrm{O}_{3}, \mathrm{~K}_{2} \mathrm{O}$ & 30000 & & 20 & 450 & 17 & & 93 & & 320 \\
\hline $\mathrm{Fe}$ & & & $\mathrm{CaO}, \mathrm{K}_{2} \mathrm{O}$ & 2000 & & 100 & 500 & 46 & & 87 & & 321 \\
\hline $\mathrm{Fe}$ & & & $\mathrm{K}$ & & & & & & & 96 & & 321 \\
\hline $\mathrm{Fe}$ & 12.4 & & $\mathrm{La}$ & 18000 & & 100 & 500 & 11 & & & & 248 \\
\hline $\mathrm{Fe}$ & 58.1 & & $\begin{array}{c}\mathrm{TiO}_{2}, \mathrm{CaO} \\
\mathrm{Al}_{2} \mathrm{O}_{3}, \mathrm{~K}_{2} \mathrm{O} \\
\mathrm{SiO}_{2}, \mathrm{Mn}\end{array}$ & & & & 700 & 100 & & & & 297 \\
\hline $\mathrm{Fe}$ & & $@ \mathrm{Al}_{2} \mathrm{O}_{3}$ & & 30000 & & 100 & 450 & 9 & & & & 162 \\
\hline $\mathrm{Fe}$ & & $@ \mathrm{CeO}_{2}$ & & 24000 & & 100 & 550 & 70 & & & & 308 \\
\hline $\mathrm{Fe}$ & & $@ \mathrm{SiO}_{2}$ & & 30000 & & 100 & 500 & 27 & 0.15 & & & 160 \\
\hline $\mathrm{Fe}$ & & $@ \mathrm{SiO}_{2}$ & & 120000 & & 100 & 400 & 10 & & & & 305 \\
\hline $\mathrm{Fe}$ & & $@ \mathrm{SiO}_{2}$ & & 30000 & & 100 & 450 & 8 & 0.05 & 69 & & 306 \\
\hline $\mathrm{Fe}$ & & $@ \mathrm{SiO}_{2}$ & Cs & 30000 & & 100 & 450 & 17 & 0.09 & 61 & & 306 \\
\hline $\mathrm{Fe}$ & & $\underset{\mathrm{Cs}}{@ \mathrm{SiO}_{2}-}$ & & 30000 & & 100 & 450 & 16 & 0.09 & 63 & & 306 \\
\hline $\mathrm{Fe}$ & & $@ \mathrm{TiO}_{2}$ & & 24000 & & 100 & 550 & 60 & & & & 308 \\
\hline $\mathrm{Fe}$ & 13 & $\mathrm{AC}$ & & & 45000 & 0.2 & 750 & 90 & & & & 244 \\
\hline $\mathrm{Fe}$ & & $\mathrm{Al}_{2} \mathrm{O}_{3}$ & & 60 & & 5 & 400 & 77 & & & & 312 \\
\hline $\mathrm{Fe}$ & 1 & $\mathrm{Al}_{2} \mathrm{O}_{3}$ & & & & 100 & 580 & & & & 0.33 & 155 \\
\hline $\mathrm{Fe}$ & 10 & $\mathrm{Al}_{2} \mathrm{O}_{3}$ & & 240000 & 80000 & 50 & 500 & 25 & & & & 218 \\
\hline $\mathrm{Fe}$ & 90 & $\mathrm{Al}_{2} \mathrm{O}_{3}$ & & 36000 & & 100 & 600 & 86 & & 127 & & 245 \\
\hline $\mathrm{Fe}$ & 5.1 & $\begin{array}{l}\text { C/SBA- } \\
15\end{array}$ & & 60000 & & 100 & 600 & 32 & & & & 288 \\
\hline $\mathrm{Fe}$ & 5.9 & carbon & & & 45000 & 0.2 & 750 & 96 & & & & 249 \\
\hline $\mathrm{Fe}$ & & $\mathrm{CeO}_{2}$ & & 60 & & 5 & 400 & 88 & & & & 312 \\
\hline $\mathrm{Fe}$ & & $\begin{array}{r}\mathrm{CeO}_{2} / \\
\mathrm{ZrO}_{2}\end{array}$ & & 60 & & 100 & 400 & 97 & & 21 & & 312 \\
\hline $\mathrm{Fe}$ & 12.2 & CMK-5 & & 60000 & & 100 & 600 & 74 & & & & 288 \\
\hline $\mathrm{Fe}$ & 3.5 & CNFs & & 6500 & & 100 & 600 & 51 & & & & 317 \\
\hline $\mathrm{Fe}$ & 3.2 & $\begin{array}{l}\text { CNFs/ } \\
\text { CMFs }\end{array}$ & & 18000 & & 100 & 550 & 15 & & & & 315 \\
\hline $\mathrm{Fe}$ & 3.5 & $\begin{array}{l}\text { CNFs- } \\
\text { mica }\end{array}$ & & 6500 & & 100 & 600 & 99 & & 94 & & 317 \\
\hline $\mathrm{Fe}$ & & CNTs & & 30000 & & 100 & 500 & 2 & 0.01 & 149 & & 156 \\
\hline $\mathrm{Fe}$ & 2.8 & CNTs & & & 5000 & 100 & 700 & 75 & & & & 163 \\
\hline $\mathrm{Fe}$ & 5 & CNTs & & 6000 & & 100 & 500 & 15 & & 142 & & 247 \\
\hline $\mathrm{Fe}$ & 5 & CNTs & & 36000 & & 100 & 550 & & 0.07 & 147 & & 322 \\
\hline $\mathrm{Fe}$ & 66 & CNTs & & & & & & & & & & 233 \\
\hline $\mathrm{Fe}$ & 5.9 & coal char & & & & 0.2 & 750 & 96 & & & & 123 \\
\hline $\mathrm{Fe}$ & 1.29 & GC & & 6000 & & 100 & 600 & 71 & & & & 313 \\
\hline $\mathrm{Fe}$ & 24 & GC & $\mathrm{K}$ & 375000 & & 20 & 400 & & & 166 & 0.016 & 314 \\
\hline $\mathrm{Fe}$ & 20 & $\mathrm{La}-\mathrm{MgO}$ & & 22000 & & 100 & 400 & 3 & & 197 & $0.002^{a}$ & 246 \\
\hline $\mathrm{Fe}$ & 48 & $\mathrm{MgO}$ & & 120000 & & 3 & 450 & 6 & & & & 311 \\
\hline $\mathrm{Fe}$ & 24 & $\mathrm{MgO}$ & $\mathrm{Al}$ & 120000 & & 3 & 450 & 3 & & & & 311 \\
\hline $\mathrm{Fe}$ & 28 & $\mathrm{MgO}$ & $\mathrm{Ga}$ & 120000 & & 3 & 450 & 24 & & 70 & & 311 \\
\hline $\mathrm{Fe}$ & 3.5 & Mica & & 6500 & & 100 & 600 & 85 & & & & 317 \\
\hline
\end{tabular}


Table 5. continued

\begin{tabular}{|c|c|c|c|c|c|c|c|c|c|c|c|c|}
\hline active phase & wt $\%$ & support & promoter & $\begin{array}{c}\text { WHSV } \\
\left(\mathrm{mL} \mathrm{g}^{-1} \mathrm{~h}^{-1}\right)\end{array}$ & $\begin{array}{c}\text { GHSV } \\
\left(\mathrm{h}^{-1}\right)\end{array}$ & $\begin{array}{c}\% \mathrm{NH}_{3} \\
\text { inlet } \\
\text { flow }\end{array}$ & $T\left({ }^{\circ} \mathrm{C}\right)$ & $\begin{array}{c}\text { conv } \\
(\%)\end{array}$ & $\begin{array}{c}\mathrm{H}_{2} \text { rate } \\
\left(\mathrm{mmol} \mathrm{g}^{-1} \mathrm{~s}^{-1}\right)\end{array}$ & $\begin{array}{c}E_{\mathrm{a}} \\
\left(\mathrm{kJ} \mathrm{mol}^{-1}\right)\end{array}$ & $\begin{array}{l}\text { TOF } \\
\left(\mathrm{s}^{-1}\right)\end{array}$ & ref \\
\hline $\mathrm{Fe}$ & 31 & SBA-15 & & 15000 & & 100 & 550 & 18 & & & & 298 \\
\hline $\mathrm{Fe}$ & & $\mathrm{SiO}_{2}$ & & 60 & & 5 & 400 & 61 & & & & 312 \\
\hline $\mathrm{Fe}$ & & $\mathrm{SrO}$ & & 60 & & 5 & 400 & 61 & & & & 312 \\
\hline $\mathrm{Fe}$ & 10 & $\begin{array}{r}\mathrm{Y}_{2} \mathrm{O}_{3}- \\
\mathrm{ZrO}_{2}\end{array}$ & & 46000 & & 100 & 550 & 6 & & & & 323 \\
\hline $\mathrm{Fe}$ & & $\mathrm{ZrO}_{2}$ & & 60 & & 100 & 400 & 57 & & & & 312 \\
\hline $\mathrm{Fe}$ & 5 & ZSM-5 & & 30000 & & 100 & 500 & 13 & & & & 307 \\
\hline
\end{tabular}

$\mathrm{Zr}>\mathrm{Sr}>\mathrm{Y}^{277}$ Deng et al. ${ }^{278}$ modified the support of a Ni/CeO catalyst with $\mathrm{ZrO}_{2}$, obtaining an increase in conversion of $30 \%$ compared to the catalyst with the unmodified support, and an increase of almost three times compared to a catalyst composed of $\mathrm{Ni} / \mathrm{Al}_{2} \mathrm{O}_{3}$ with the same metal content. A Ni alumina catalyst modified with $\mathrm{CeO}_{2}-\mathrm{ZrO}_{2}$ was tested by Sima et al., ${ }^{279}$ obtaining a remarkable increase in catalytic activity. Other modifications of the alumina support were tested by Zhao et al., ${ }^{280}$ who prepared a catalyst composed by $\mathrm{Ni}$ introduced into the hydrocalumite double-layered hydroxide structure. Similarly, Su et al. ${ }^{223}$ tested a $\mathrm{Mg}-\mathrm{Al}$ double-layered hydroxide as a support for $\mathrm{Ni}$ and concluded that its activity was similar to that of $\mathrm{Ru} / \mathrm{Al}_{2} \mathrm{O}_{3}$, but did not exceed the one obtained with $\mathrm{Ru}$ / $\mathrm{Al}_{2} \mathrm{O}_{3}$ promoted with $\mathrm{K}$. The activity of the $\mathrm{Ni} / \mathrm{MgO}$ catalyst was compared with that of $\mathrm{Ni}$ supported on different compositions of $\mathrm{MgO}$ and $\mathrm{Al}_{2} \mathrm{O}_{3}$ by Sato et al. ${ }^{281}$ Using a zeolite support for $\mathrm{Ni}$, Inokawa et al. ${ }^{282}$ obtained a conversion three times higher than that of a conventional $\mathrm{Ni} / \mathrm{Al}_{2} \mathrm{O}_{3}$ catalyst. The same authors reported that by changing the catalyst preparation method they were able to reduce the size of the $\mathrm{Ni}$ particles from 50 to $5 \mathrm{~nm}$, obtaining significantly better catalytic results ( 32 vs $61 \%$ ammonia conversion at $500{ }^{\circ} \mathrm{C}$ ).

Carbon-based supports have also been tested in the case of $\mathrm{Ni}$, although they do not show the good synergy with the metal as observed for Ru. Carbon nanotubes (CNTs) and graphene were first tested as supports by Alhamed et al., ${ }^{283}$ but ammonia conversion was only obtained by using Ni supported on CNTs, which in turn presented higher conversion values compared to $\mathrm{Ni} / \mathrm{AC}^{284}$ By using oxidized graphene (GO) as a support for $\mathrm{Ni}$, Meng et al. ${ }^{285}$ reported a higher catalytic activity compared to $\mathrm{Ni}$ supported on ordered mesoporous carbon or activated carbon ( 17 vs 8 and $2 \%$ at $500{ }^{\circ} \mathrm{C}$, respectively). Finally, red mud has also been used as a low cost support for $\mathrm{Ni}^{286,287}$

3.1.3.2. Iron. As already mentioned, catalysts active for the Haber-Bosch synthesis of ammonia, such as the iron catalyst promoted with $\mathrm{K}_{2} \mathrm{O}, \mathrm{Al}_{2} \mathrm{O}_{3}$, and $\mathrm{CaO}$, were initially tested to decompose ammonia. ${ }^{294,295}$ A catalyst prepared by melting magnetite with $\mathrm{Al}_{2} \mathrm{O}_{3}$ and $\mathrm{CaO}$ resulted in good catalytic activity. ${ }^{296}$ A catalyst composed by a natural iron mineral with impurities of $\mathrm{TiO}_{2}, \mathrm{CaO}, \mathrm{Al}_{2} \mathrm{O}_{3}, \mathrm{~K}_{2} \mathrm{O}, \mathrm{SiO}_{2}$, and $\mathrm{Mn}$ showed good activity, but is unstable over time, with a rapid decrease in a $3 \mathrm{~h}$ test. ${ }^{297}$ The same behavior was obtained with a reference catalyst composed only of $\mathrm{Fe}_{2} \mathrm{O}_{3}{ }^{297}$ Through in situ experiments, Tseng et al. ${ }^{298}$ claimed that the active form of catalysts composed of $\mathrm{Fe}$ is $\mathrm{Fe}_{3} \mathrm{~N}_{x}$, while at high temperatures $\left(>675^{\circ} \mathrm{C}\right.$ ) $\mathrm{FeN}_{x}$ is formed, which has a negative influence on ammonia conversion. $\mathrm{A} \mathrm{Fe}_{2} \mathrm{O}_{3}$ catalyst supported on SBA-15 mesoporous silica shows better catalytic activity than the bare iron oxide (18 vs $4 \%$ at $\left.500{ }^{\circ} \mathrm{C}\right) .{ }^{298}$ Pelka et al. ${ }^{299}$ observed that the catalytic decomposition reaction rate of ammonia is higher in the case of nanocrystalline iron compared to iron nitride $\mathrm{Fe}_{4} \mathrm{~N}$. The decrease of ammonia conversion in the presence of $\mathrm{Fe}_{4} \mathrm{~N}$ has also been evidenced with a Fe catalyst promoted with $\mathrm{Al}_{2} \mathrm{O}_{3}$, $\mathrm{CaO}$, and $\mathrm{K}_{2} \mathrm{O}$ with or without $\mathrm{SiO}_{2} \cdot{ }^{300-303}$ Temperature influences the degree of nitriding, being favored at temperatures above $400{ }^{\circ} \mathrm{C} .{ }^{304}$ Also in the case of iron, encapsulation in an oxide has been shown to improve the catalytic activity of the catalyst. $\mathrm{Li}$ et al. ${ }^{160}$ compared $\mathrm{Fe}$ nanoparticles alone and encapsulated in silica, finding that the latter are considerably more active (conversion of 9 vs $27 \%$ at $500{ }^{\circ} \mathrm{C}$ ). The encapsulation of $\mathrm{Fe}$ particles in $\mathrm{SiO}_{2}$ has been demonstrated to significantly increase the stability of $\mathrm{Fe}$ for the reaction. ${ }^{305}$ Adding Cs to this catalyst, $\mathrm{Li}$ et al. obtained around twice the conversion of ammonia at $450{ }^{\circ} \mathrm{C}$ with respect to the catalyst without promoting. ${ }^{306}$ Also, by encapsulating Fe nanoparticles in $\mathrm{Al}_{2} \mathrm{O}_{3}$, the catalytic activity showed an increase with respect to $\mathrm{SiO}_{2}$ under the same reaction conditions $\left(9\right.$ vs $4 \%$ at $\left.450{ }^{\circ} \mathrm{C}\right) .{ }^{162}$

By varying the proportions between the oxides of $\mathrm{Si}$ and $\mathrm{Al}$, the acid-base characteristics of the support and the interaction between the metal and the support can be modulated, obtaining an improvement in the conversion of ammonia by increasing the basicity of the catalyst. ${ }^{307}$ In this sense, Cui et al. ${ }^{308}$ compared the activity of iron oxide alone or modified with cerium or titanium oxide, obtaining a higher and more stable conversion over time in the case of composite catalysts. It is worth mentioning that the modification with ceria was the most effective. Using natural limonite, a $\mathrm{Fe}$ mineral with small amounts of Si, Al, Ca, and Mg, Tsubouchi et al. ${ }^{309}$ obtained good conversion values and proposed that the decomposition of ammonia catalyzed with $\mathrm{Fe}$ proceeds through a cyclic mechanism that involves both metallic iron and its nitride form. Similarly, a series of $\mathrm{Fe}$ catalysts supported on $\mathrm{MgO}$ and promoted with Ga were prepared and tested for the decomposition of ammonia, obtaining good activity compared to a catalyst composed only of $\mathrm{Fe},{ }^{310}$ and also with respect to $\mathrm{Fe} /$ $\mathrm{MgO}$ and $\mathrm{Fe} / \mathrm{MgO}$ promoted with $\mathrm{Al}^{311}$ Other supports that have been used for $\mathrm{Fe}$ are $\mathrm{SiO}_{2}, \mathrm{Al}_{2} \mathrm{O}_{3}, \mathrm{CeO}_{2}, \mathrm{SrO}, \mathrm{ZrO}_{2}$, and $\mathrm{CeO}_{2}-\mathrm{ZrO}_{2}$, obtaining the highest conversion with the ceriazirconia mixture. ${ }^{312}$ It is important to mention that $40 \%$ of the $\mathrm{N}_{2}$ produced during the reaction was incorporated into the catalyst to form $\mathrm{Fe}_{2} \mathrm{~N}$.

The synergy between $\mathrm{Fe}$ and carbon-based supports are superior to the case of nickel. Supported on ordered mesoporous carbon CMK-5, Fe showed superior catalytic activity compared to $\mathrm{Ni} / \mathrm{Al}_{2} \mathrm{O}_{3}$ and to $\mathrm{Fe}$ supported on a mixture of carbon and SBA-15 (74 vs 32 and $29 \%$ at $600{ }^{\circ} \mathrm{C}$, respectively). ${ }^{288}$ Moreover, high activity and stability can be obtained when graphitized carbon (GC) is used as support for Fe. ${ }^{313}$ When $\mathrm{K}$ is added to a Fe catalyst supported on GC, the catalytic activity in the decomposition of ammonia increases. ${ }^{314}$ In a similar way, Ji 
Table 6. Catalysts Based on Cobalt As Active Phase Used to Decompose Ammonia and Their Catalytic Performances at 1 atm

\begin{tabular}{|c|c|c|c|c|c|c|c|c|c|c|c|c|}
\hline $\begin{array}{l}\text { active } \\
\text { phase }\end{array}$ & wt $\%$ & support & promoter & $\begin{array}{c}\text { WHSV } \\
\left(\mathrm{mL} \mathrm{g}^{-1} \mathrm{~h}^{-1}\right)\end{array}$ & $\begin{array}{c}\text { GHSV } \\
\left(\mathrm{h}^{-1}\right)\end{array}$ & $\begin{array}{l}\% \mathrm{NH}_{3} \\
\text { inlet flow }\end{array}$ & $\begin{array}{c}T \\
\left({ }^{\circ} \mathrm{C}\right)\end{array}$ & $\begin{array}{c}\text { conv } \\
(\%)\end{array}$ & $\begin{array}{c}\mathrm{H}_{2} \text { rate } \\
\left(\mathrm{mmol} \mathrm{g}^{-1} \mathrm{~s}^{-1}\right)\end{array}$ & $\begin{array}{c}E_{\mathrm{a}} \\
\left(\mathrm{kJ} \mathrm{mol}{ }^{-1}\right)\end{array}$ & $\begin{array}{l}\text { TOF } \\
\left(\mathrm{s}^{-1}\right)\end{array}$ & ref \\
\hline Co & & & & 18000 & & 100 & 500 & 85 & 0.003 & & & 248 \\
\hline Co & & & & 18000 & & 100 & 500 & 21 & & & & 325 \\
\hline Co & & & & 18000 & & 100 & 500 & 18 & & & & 327 \\
\hline Co & & & & 22000 & & 100 & 400 & 28 & & & & 246 \\
\hline Co & & & & 24000 & & 100 & 500 & 5 & 0.02 & & & 337 \\
\hline Co & & & & & & 15 & 550 & 25 & & & & 318 \\
\hline Co & & & $\mathrm{Al}_{2} \mathrm{O}_{3}, \mathrm{CaO}, \mathrm{K}_{2} \mathrm{O}$ & 24000 & & 100 & 500 & 40 & 0.14 & & & 337 \\
\hline $\mathrm{Co}_{3} \mathrm{O}_{4}$ & & & $\mathrm{CaO}, \mathrm{Al}_{2} \mathrm{O}_{3}, \mathrm{~K}_{2} \mathrm{O}$ & 19544 & & 100 & 500 & 85 & & & & 326 \\
\hline Co & 6.8 & & $\mathrm{La}$ & 18000 & & 100 & 500 & 63 & & & & 248 \\
\hline Co & & $@ \mathrm{SiO}_{2}$ & & 30000 & & 100 & 450 & 4 & & & & 162 \\
\hline Co & 5 & AC & & & 6000 & 100 & 500 & 34 & & & & 329 \\
\hline Co & 7.0 & $\mathrm{AC}$ & & 5200 & 33 & 100 & 450 & 2 & & & & 226 \\
\hline Co & 1 & $\mathrm{Al}_{2} \mathrm{O}_{3}$ & & & & 100 & 580 & & & & 1.33 & 155 \\
\hline Co & 5 & $\mathrm{Al}_{2} \mathrm{O}_{3}$ & & 36000 & & 100 & 500 & 21 & & & & 338 \\
\hline Co & 90 & $\mathrm{Al}_{2} \mathrm{O}_{3}$ & & 36000 & & 100 & 600 & 100 & & 123 & & 245 \\
\hline Co & 90 & $\mathrm{Al}_{2} \mathrm{O}_{3}$ & & 18000 & & 100 & 500 & 44 & 0.9 & 148 & & 325 \\
\hline Co & 90 & $\mathrm{Al}_{2} \mathrm{O}_{3}$ & & 18000 & & 100 & 500 & 57 & & & & 327 \\
\hline Co & 7.0 & $A X-21$ & & 5200 & 33 & 100 & 450 & 25 & & 89 & & 226 \\
\hline Co & 7.0 & $A X-21$ & Cs & 5200 & 33 & 100 & 450 & 3 & & & & 226 \\
\hline Co & 10 & $\mathrm{Ce}_{0.6} \mathrm{Zr}_{0.3} \mathrm{Y}_{0.1} \mathrm{O}_{2}$ & & 6000 & & 100 & 350 & 7 & 0.008 & 50 & 0.54 & 292 \\
\hline Co & 10 & $\mathrm{CeO}_{2}$ & & 6000 & & 100 & 450 & 30 & & & & 292 \\
\hline Co & 4.1 & CNTs & & & 20000 & 100 & 700 & 100 & & & & 163 \\
\hline Co & 5 & CNTs & & 6000 & & 100 & 500 & 61 & & 93 & & 247 \\
\hline Co & 5 & CNTs & & 36000 & & 100 & 550 & & 0.18 & 79 & & 322 \\
\hline Co & 5 & CNTs & & & & & & & & & & 233 \\
\hline Co & 7.0 & CNTs & & 5200 & 33 & 100 & 450 & 8 & & 94 & & 226 \\
\hline Co & 7.0 & CNTs & Cs & 5200 & 33 & 100 & 450 & 9 & & & & 226 \\
\hline Co & 20 & $\mathrm{La}-\mathrm{MgO}$ & & 22000 & & 100 & 400 & 37 & & 167 & $0.009^{a}$ & 246 \\
\hline Co & 5 & MCM-41 & & 36000 & & 100 & 500 & 28 & & & & 339 \\
\hline Co & 7.0 & MESO-C & & 5200 & 33 & 100 & 450 & 10 & & 104 & & 226 \\
\hline Co & 20 & $\mathrm{MgO}$ & & 22000 & & 100 & 400 & 25 & & & & 246 \\
\hline Co & 5 & $\mathrm{MgO}-\mathrm{Al}_{2} \mathrm{O}_{3}$ & & & 6000 & 100 & 550 & 32 & & 192 & & 331 \\
\hline Co & 5 & $\mathrm{MgO}-\mathrm{CeO}_{2}$ & & & 6000 & 100 & 550 & 69 & & 81 & & 331 \\
\hline Co & 5 & $\mathrm{MgO}-\mathrm{La}_{2} \mathrm{O}_{3}$ & & & 6000 & 100 & 550 & 92 & & 67 & & 332 \\
\hline Co & 7.0 & MSC-30 & & 5200 & 33 & 100 & 450 & 12 & & 102 & & 226 \\
\hline Co & 7.0 & MSC-30 & Cs & 5200 & 33 & 100 & 450 & 10 & & & & 226 \\
\hline Co & 10 & MWCNTs & & & 6000 & 100 & 500 & 75 & & 69 & & 328 \\
\hline Co & 5 & MWCNTs & & & 6000 & 100 & 500 & 61 & & & & 329 \\
\hline Co & 5 & RGO & & & 6000 & 100 & 500 & 4 & & & & 329 \\
\hline Co & & silicate & & 150000 & & 100 & 600 & 51 & 1.2 & & & 335 \\
\hline Co & & silicate & $\mathrm{KOH}$ & 150000 & & 100 & 600 & 67 & 1.7 & & & 335 \\
\hline Co & 42 & $\mathrm{SiO}_{2}$ & & & & & & & & 155 & & 334 \\
\hline Co & & sodium silicate & & 150000 & & 100 & 600 & 71 & 1.6 & & & 336 \\
\hline Co & 5 & SWCNTs & & & 6000 & 100 & 500 & 2 & & & & 329 \\
\hline Co & 6.5 & Ti-NT & & 6000 & & 30 & 550 & 25 & & 85 & & 330 \\
\hline Co & 14 & Ti-NT & $\mathrm{Na}$ & 6000 & & 30 & 550 & 41 & & 89 & & 330 \\
\hline Co & 10 & $\mathrm{Y}_{2} \mathrm{O}_{3}$ & & 6000 & & 100 & 450 & 28 & & & & 292 \\
\hline Co & 10 & $\mathrm{ZrO}_{2}$ & & 6000 & & 100 & 450 & 19 & & & & 292 \\
\hline
\end{tabular}

et al. ${ }^{315,316}$ tested Fe catalysts supported on carbon nanofibers (CNFs), obtaining an outstanding catalytic activity. Duan et al. $^{317}$ compared the $\mathrm{Fe} / \mathrm{CNF}$ s catalyst with a $\mathrm{Fe}$ catalyst supported on mica and a mixture of the two materials. The Fe/ CNFs-mica catalyst showed superior activity and stability, which could be related to a better dispersion of the Fe particles. Ohtsuka et al. ${ }^{123}$ used $\mathrm{Fe}$ and Ca catalysts supported on lignite doped with $\mathrm{Fe}$ and $\mathrm{Ca}$ ions and obtained a higher ammonia conversion with respect to a Fe catalyst supported on activated carbon. Finally, an organic compound, $\mathrm{Fe}(\mathrm{II})$ phthalocyanine $\left(\mathrm{C}_{32} \mathrm{H}_{16} \mathrm{FeN}_{8}\right)$, was used in the ammonia decomposition reaction, obtaining higher conversion of ammonia than with a commercial $\mathrm{Ni} / \mathrm{Al}_{2} \mathrm{O}_{3}$ catalyst $\left(33\right.$ vs $17 \%$ at $\left.600{ }^{\circ} \mathrm{C}\right) .^{289}$ Catalysts based on iron as an active phase used to decompose ammonia and their catalytic performances are reported in Table 5 .

3.1.3.3. Cobalt. Although cobalt is less active than iron in the ammonia synthesis, it is more efficient in the ammonia 
Table 7. Catalysts Based on Molybdenum as Active Phase Used to Decompose Ammonia and Their Catalytic Performances at 1 atm

\begin{tabular}{|c|c|c|c|c|c|c|c|c|c|c|c|c|}
\hline $\begin{array}{l}\text { active } \\
\text { phase }\end{array}$ & wt $\%$ & support & promoter & $\begin{array}{c}\text { WHSV } \\
\left(\mathrm{mL} \mathrm{g}^{-1} \mathrm{~h}^{-1}\right)\end{array}$ & $\begin{array}{c}\text { GHSV } \\
\left(\mathrm{h}^{-1}\right)\end{array}$ & $\begin{array}{c}\% \mathrm{NH}_{3} \text { inlet } \\
\text { flow }\end{array}$ & $\begin{array}{c}\mathrm{T} \\
\left({ }^{\circ} \mathrm{C}\right)\end{array}$ & $\begin{array}{c}\text { conv } \\
(\%)\end{array}$ & $\begin{array}{c}\mathrm{H}_{2} \text { rate } \\
\left(\mathrm{mmol} \mathrm{g}^{-1} \mathrm{~s}^{-1}\right)\end{array}$ & $\begin{array}{c}E_{\mathrm{a}} \\
\left(\mathrm{kJ} \mathrm{mol}{ }^{-1}\right)\end{array}$ & $\begin{array}{l}\text { TOF } \\
\left(\mathrm{s}^{-1}\right)\end{array}$ & ref \\
\hline Mo & & & & 15000 & & 100 & 500 & 13 & & & & 340 \\
\hline Mo & 5 & $\mathrm{Al}_{2} \mathrm{O}_{3}$ & & 36000 & & 100 & 500 & 22 & & & & 338 \\
\hline Mo & & $\mathrm{C}$ & & 15000 & & 100 & 600 & 66 & & 137 & & 344 \\
\hline Mo & 5 & MCM-41 & & 36000 & & 100 & 500 & 28 & & & & 339 \\
\hline Mo & 10 & $\begin{array}{r}\mathrm{Y}_{2} \mathrm{O}_{3}- \\
\mathrm{ZrO}_{2}\end{array}$ & & 46000 & & 100 & 550 & 10 & & & & 323 \\
\hline $\mathrm{Mo}_{2} \mathrm{C}$ & & & & & 7500 & 100 & 570 & & & 151 & 18.3 & 242 \\
\hline $\mathrm{Mo}_{2} \mathrm{C}$ & & & & 36000 & & 100 & 600 & 77 & & 89 & & 236 \\
\hline $\mathrm{Mo}_{2} \mathrm{~N}$ & & & & 22000 & & 100 & 550 & 94 & 0.4 & 139 & & 342 \\
\hline $\mathrm{Mo}_{2} \mathrm{~N}$ & & & & & 170000 & 100 & 500 & 10 & & 148 & & 345 \\
\hline $\mathrm{Mo}_{2} \mathrm{~N}$ & & & & 15000 & & 100 & 500 & 100 & & & & 346 \\
\hline $\mathrm{Mo}_{2} \mathrm{~N}$ & & & & & 6000 & 100 & 500 & 27 & & 131 & & 347 \\
\hline $\mathrm{Mo}_{2} \mathrm{~N}$ & & & & 6000 & & 100 & 550 & 72 & & 131 & & 348 \\
\hline $\mathrm{Mo}_{2} \mathrm{~N}$ & & & & 6000 & & 100 & 550 & 72 & & 131 & & 349 \\
\hline $\mathrm{Mo}_{2} \mathrm{~N}$ & & & & 6000 & & 100 & 550 & 69 & & 97 & & 350 \\
\hline $\mathrm{Mo}_{2} \mathrm{~N}$ & & & & & 6000 & 100 & 500 & 27 & & 131 & & 351 \\
\hline $\mathrm{MoN}$ & & & & & 7500 & 100 & 570 & & & 151 & 12.6 & 352 \\
\hline $\mathrm{MoN}$ & 25.9 & $\mathrm{C}$ & & 15000 & & 100 & 600 & 89 & & 124 & & 344 \\
\hline $\mathrm{MoN}$ & 51 & SBA-15 & & 60000 & & 100 & 500 & 62 & & & & 157 \\
\hline $\mathrm{MoN}$ & 80 & $\mathrm{SiO}_{2}$ & & 60000 & & 100 & 500 & 50 & & & & 157 \\
\hline $\mathrm{MoN}_{x}$ & 10 & $\mathrm{Al}_{2} \mathrm{O}_{3}$ & & & 1800 & 100 & 650 & 99 & & & & 290 \\
\hline $\mathrm{MoS}_{2}$ & 5.7 & Al-laponite & & & 19200 & 10 & 600 & 46 & 0.23 & & 4.1 & 341 \\
\hline $\mathrm{MoS}_{2}$ & 6.0 & laponite & & & 24000 & 10 & 600 & 35 & 0.16 & & 3.6 & 341 \\
\hline $\mathrm{MoS}_{2}$ & 5.9 & Ti-laponite & & & 16000 & 10 & 600 & 75 & 0.24 & & 4.0 & 341 \\
\hline $\mathrm{MoS}_{2}$ & 6.1 & Zr-laponite & & & 16000 & 10 & 600 & 94 & 0.29 & & 4.5 & 341 \\
\hline
\end{tabular}

decomposition reaction. ${ }^{324}$ However, cobalt in its oxide form does not show good catalytic activity in the decomposition of ammonia. ${ }^{325}$ In the work of Zhang et al. the conversion of ammonia using $\mathrm{Co}_{3} \mathrm{O}_{4}$ reaches its maximum at a reaction temperature of $500{ }^{\circ} \mathrm{C}$, while at higher temperatures the conversion decreases. ${ }^{325}$ Czekajło and Lendzion-Bielun ${ }^{326}$ were able to increase the conversion by adding the oxides of $\mathrm{Al}, \mathrm{Ca}$, and $\mathrm{K}$ as promoters. The addition of promoter oxides is believed to stabilize the surface area of the catalysts. Therefore, by adding a small amount of alumina (10 wt \%) to cobalt oxide, the conversion of ammonia increased significantly. ${ }^{325}$ Using a catalyst with the same composition, Gu et al. ${ }^{327}$ obtained a similar result; the conversion increased by around $20 \%$ compared to pure cobalt oxide.

Using cobalt supported on different forms of carbon (CNTs, AX-21, MSC-30, MESO-C, and AC), Torrente-Murciano et al. $^{226}$ found that the use of Cs as a promoter causes a decrease in conversion compared to the nonpromoted form, which constitutes an opposite behavior with respect to $\mathrm{Ru}$ catalysts supported on carbon. ${ }^{185}$ Co was also studied supported on carbon nanotubes, showing good catalytic activity. ${ }^{328}$ In a comparison of different carbon supports for Co, multiwalled carbon nanotubes (MWCNTs) showed better results than activated carbon (AC), reduced graphene oxide (RGO), and single-walled carbon nanotubes (SWCNTs). ${ }^{329}$

Lara-García et al. ${ }^{330}$ demonstrated the promoting effect of $\mathrm{Na}$ on a cobalt catalyst supported on titania nanotubes. They observed that the best catalytic results were obtained with the catalyst with Co particles of $15 \mathrm{~nm}$, which is in agreement with the literature that reports the highest activity for Co nanoparticles with dimensions between 10 and $20 \mathrm{~nm} .{ }^{144}$ Podila et al. $^{331}$ studied cobalt catalysts supported on different mixed oxide systems of $\mathrm{MgO}$ with $\mathrm{Al}_{2} \mathrm{O}_{3}, \mathrm{La}_{2} \mathrm{O}_{3}$, and $\mathrm{CeO}_{2}$, obtaining the following order of the catalytic activity: $\mathrm{La}_{2} \mathrm{O}_{3}>\mathrm{CeO}_{2}>\mathrm{Al}_{2} \mathrm{O}_{3}$. The best catalytic activity of the $\mathrm{Co} / \mathrm{MgO}-\mathrm{La}_{2} \mathrm{O}_{3}$ catalyst was attributed to its higher basicity compared to other formulations, and different preparations were tested to optimize its activity. ${ }^{332,333}$ Similarly, $\mathrm{Hu}$ et al. $^{246}$ verified the promoting effect of La on the activity of Co supported on $\mathrm{MgO}$. The conversion of pure ammonia at $400{ }^{\circ} \mathrm{C}$ increases by $12 \%$ with respect to the nonpromoted catalyst. A Co catalyst supported on $\mathrm{SiO}_{2}$ was also tested by $\mathrm{Hu}$ et al. ${ }^{334}$ Co shows good activity when silicates are used as support. In this sense, the use of cobalt incorporated into a structured mesoporous silicate increased notably the ammonia conversion. ${ }^{335}$ Similarly, cobalt incorporated in the structure of a sodium silicate showed high activity. ${ }^{336}$ Catalysts based on cobalt as the active phase used to decompose ammonia and their catalytic performances are reported in Table 6.

3.1.3.4. Molybdenum. Another non-noble metal that has been studied for the decomposition of ammonia is Mo. Catalysts based on molybdenum as the active phase used to decompose ammonia and their catalytic performances are reported in Table 7. Molybdenum oxide $\left(\mathrm{MoO}_{3}\right)$ showed low catalytic activity, but it could be improved through a mechanochemical pretreatment (ball milling). ${ }^{340}$ In successive cycles, the catalyst increased its catalytic activity due to the formation of molybdenum nitride, which is catalytically active and is formed during the reaction. Santhana Krishnan et al. ${ }^{341}$ tested catalysts with $\mathrm{MoS}_{2}$ as the active phase supported on laponite and laponite modified with $\mathrm{Al}, \mathrm{Ti}$, and $\mathrm{Zr}$, obtaining the best results with the support modified with $\mathrm{Zr}$, reaching a conversion of $94 \%$ at $600{ }^{\circ} \mathrm{C}$. They concluded that the modification of the support with heteroatoms allows an increase in both the dispersion of $\mathrm{MoS}_{2}$ and the basicity of the catalyst. Xu et al. ${ }^{342}$ synthesized a $\mathrm{Mo}_{2} \mathrm{~N}$ catalyst from molybdenum oxide nanobelts, which showed an 
Table 8. Catalysts Based on Carbides and Nitrides of Metals Other than Molybdenum Used to Decompose Ammonia and Their Catalytic Performances at 1 atm

\begin{tabular}{|c|c|c|c|c|c|c|c|c|c|c|c|}
\hline $\begin{array}{l}\text { active } \\
\text { phase }\end{array}$ & wt $\%$ & support promoter & $\begin{array}{c}\text { WHSV } \\
\left(\mathrm{mL} \mathrm{g}^{-1} \mathrm{~h}^{-1}\right)\end{array}$ & $\begin{array}{c}\text { GHSV } \\
\left(\mathrm{h}^{-1}\right)\end{array}$ & $\begin{array}{c}\% \mathrm{NH}_{3} \text { inlet } \\
\text { flow }\end{array}$ & $\begin{array}{c}T \\
\left({ }^{\circ} \mathrm{C}\right)\end{array}$ & $\begin{array}{c}\text { conv } \\
(\%)\end{array}$ & $\begin{array}{c}\mathrm{H}_{2} \text { rate } \\
\left(\mathrm{mmol} \mathrm{g}^{-1} \mathrm{~s}^{-1}\right)\end{array}$ & $\underset{(\mathrm{kJ} \mathrm{mol}}{\left.E_{\mathrm{a}}\right)}$ & $\begin{array}{l}\text { TOF } \\
\left(\mathrm{s}^{-1}\right)\end{array}$ & ref \\
\hline $\mathrm{CrN}$ & & & 60000 & & 100 & 500 & 0 & & & & 233 \\
\hline $\mathrm{Fe}_{2} \mathrm{~N}$ & & & 60000 & & 100 & 500 & 4 & & & & 359 \\
\hline $\mathrm{Fe}_{2} \mathrm{~N}$ & & & & & & & & & 181 & & 233 \\
\hline $\mathrm{Fe}_{3} \mathrm{C}$ & & & 15000 & & 100 & 550 & 23 & & & & 355 \\
\hline $\mathrm{MnN}$ & & & 60000 & & 5 & 500 & 15 & & & & 360 \\
\hline $\mathrm{MnN}$ & & & 60000 & & 100 & 500 & 3 & & & & 233 \\
\hline $\mathrm{TaC}$ & & & & 7500 & 100 & 570 & & & 134 & 9.24 & 354 \\
\hline TiN & & & 60000 & & 100 & 500 & 0 & & & & 233 \\
\hline $\mathrm{V}_{8} \mathrm{C}_{7}$ & & & & 7500 & 100 & 570 & & & 146 & 12.9 & 242 \\
\hline VN & & & 22000 & & 100 & 550 & 22 & & 194 & & 342 \\
\hline VN & & & & 7500 & 100 & 570 & & & 218 & 0.8 & 352 \\
\hline VN & & & & 7500 & 100 & 380 & 25 & & & & 353 \\
\hline $\mathrm{VN}$ & & & 60000 & & 100 & 500 & 1 & & & & 233 \\
\hline $\mathrm{W}_{2} \mathrm{~N}$ & & & 22000 & & 100 & 550 & 36 & & 173 & & 342 \\
\hline WC & & & 120000 & & 0.4 & 500 & 22 & & 166 & & 122 \\
\hline WC & & & 66000 & & 1.8 & 500 & 100 & & & & 357 \\
\hline WZ & & & 120000 & & 0.4 & 500 & 18 & & 209 & & 122 \\
\hline $\mathrm{ZrON}$ & & & 1500 & & 0.6 & 500 & 12 & & 187 & & 319 \\
\hline $\begin{array}{l}\mathrm{ZrON} / \\
\mathrm{ZrO}_{2}\end{array}$ & $49 / 51$ & & 750 & & 0.41 & 500 & 30 & & 106 & & 358 \\
\hline
\end{tabular}

ammonia conversion around 3 times higher compared to $\mathrm{VN}$ and $\mathrm{W}_{2} \mathrm{~N}$ nitrides under the same reaction conditions. Liu et al. $^{157}$ carried out a systematic study of a large number of supported transition metals on $\mathrm{SiO}_{2}$ and concluded that $\mathrm{MoN}_{x}$ could be a promising catalyst for the decomposition of ammonia. When the catalytic activity of $\mathrm{MoN}_{x}$ supported on $\mathrm{SiO}_{2}$ is compared with that obtained with SBA-15, a higher ammonia conversion is obtained with the second support (62 vs $50 \%$ at $500{ }^{\circ} \mathrm{C}$ ). The same authors also studied the influence of the $\mathrm{MoN}_{x} / \mathrm{SBA}-15$ catalyst preparation method to achieve its optimization. $^{343}$

Molybdenum carbide catalysts are also promising candidates for the decomposition of ammonia. Zheng et al. ${ }^{236}$ prepared and tested $\mathrm{Mo}_{2} \mathrm{C}$. The catalyst had a tendency to form $\mathrm{MoN}$ when pure ammonia was used at high temperature $\left(600{ }^{\circ} \mathrm{C}\right)$, which caused a significant loss of specific surface area. Anyhow, the catalyst was less active than $\mathrm{Ru}$ supported on graphite. ${ }^{236}$ Similarly, Li et al. ${ }^{344}$ tested catalysts composed by $\mathrm{MoO}_{2}$ and $\mathrm{Mo}_{2} \mathrm{C}$ nanoparticles dispersed in a porous carbon matrix, observing that they rapidly formed MoN under reaction, which turned out to be the active phase.

3.1.3.5. Carbides and Nitrides. Choi ${ }^{242}$ tested various forms of vanadium carbides and compared their activity with that of $\mathrm{Mo}_{2} \mathrm{C}$, showing that in all cases it is lower than that of the molybdenum catalyst, while it is notably higher than that of a catalyst composed of Pt supported on carbon. In another work, Choi et al. ${ }^{352}$ compared the activity of vanadium and molybdenum carbides with the corresponding nitrides, VN and MoN. The results showed that the carbides of the two elements are more active for the decomposition of the ammonia. However, the $\mathrm{VN}$ catalyst showed superior activity with respect to $\mathrm{Ni} / \mathrm{Al}_{2} \mathrm{O}_{3}-\mathrm{SiO}_{2} .{ }^{353}$ Also, the tantalum carbide $\mathrm{TaC}$ showed a good activity, comparable to that obtained using the other nitrides and carbides. ${ }^{354}$ Finally, using $\mathrm{Fe}_{3} \mathrm{C}$ iron carbide, Kraupner et al. ${ }^{355}$ obtained good ammonia conversion and high stability at $600{ }^{\circ} \mathrm{C}$. Pansare et al. ${ }^{122,356}$ studied the conversion of ammonia using tungsten carbide and zirconium tungstate as catalysts, obtaining a higher conversion in both cases compared to a conventional $\mathrm{Fe}$ catalyst $\left(22,18\right.$, and $11 \%$ at $500{ }^{\circ} \mathrm{C}$, respectively). A modified tungsten carbide catalyst studied by Cui et al. ${ }^{357}$ showed noticeably higher activity. Zirconium was also tested in the form of zirconium oxynitride, $\mathrm{ZrON}$, and it showed good catalytic activity in decomposing ammonia when mixed with $\mathrm{ZrO}_{2} \cdot{ }^{358}$ However, it shows limited and lower catalytic activity compared to a nonpromoted iron oxide catalyst. $^{319}$ Catalysts based on carbides and nitrides of metals other than molybdenum used to decompose ammonia and their catalytic performances are reported in Table 8 .

3.1.4. Bimetallics and Multimetallics. Among the bimetallic and multimetallic catalysts tested in the ammonia decomposition reaction, combinations of the metals $\mathrm{Co}, \mathrm{Mo}, \mathrm{Ni}, \mathrm{Fe}, \mathrm{Pt}$, $\mathrm{Cu}, \mathrm{Ir}, \mathrm{Cr}, \mathrm{Mn}, \mathrm{Mg}, \mathrm{Cu}, \mathrm{Sn}, \mathrm{Zn}, \mathrm{Li}$, and Pd have been used, and combinations of $\mathrm{Ru}$ with $\mathrm{Fe}, \mathrm{Sr}, \mathrm{Bi}, \mathrm{Pb}, \mathrm{Sn}, \mathrm{In}, \mathrm{Cd}, \mathrm{Zn}, \mathrm{Au}, \mathrm{Ag}$, $\mathrm{Cu}, \mathrm{Pt}, \mathrm{Pd}, \mathrm{Ni}$, Ir, Rh, Co, Os, Re, Mn, W, Mo, Cr, Ta, Nb, Hf, Zr, $\mathrm{Y}, \mathrm{Sc}, \mathrm{Ca}$, and $\mathrm{Mg}$ have been studied. However, the combination of cobalt and molybdenum is one of the most studied bimetallic catalysts since it exhibits significant catalytic activity. By testing an unsupported Co-Mo catalyst, Duan et al. ${ }^{361}$ observed that the active phase of the bimetallic catalyst was, indeed, $\mathrm{Co}_{3} \mathrm{Mo}_{3} \mathrm{~N}$. The bimetallic $\mathrm{Co}_{3} \mathrm{Mo}_{3} \mathrm{~N}$ catalyst showed higher conversion than the monometallic form, $\mathrm{Mo}_{2} \mathrm{~N}^{347,348}$ Also, the catalyst composed of $\mathrm{Fe}_{3} \mathrm{Mo}_{3} \mathrm{~N}$ prepared by nitriding $\mathrm{FeMoO}_{4}$ showed a higher catalytic activity than the monometallic nitride, $\mathrm{Mo}_{2} \mathrm{~N}^{349}$ Similarly, catalysts composed of Co-Mo in its nitride form promoted with $\mathrm{K}, \mathrm{Ba}, \mathrm{La}, \mathrm{Ce}$, and $\mathrm{Zr}$ were tested, showing that the promoter effect follows the order: $\mathrm{La}>\mathrm{Ba}>\mathrm{K} \approx \mathrm{Ce}$, while $\mathrm{Zr}$ exhibits a promotion effect only at a temperature higher than $500{ }^{\circ} \mathrm{C} .{ }^{362}$ Following the results obtained previously, Srifa et al. ${ }^{350}$ tested bimetallic catalysts of Mo combined with $\mathrm{Co}, \mathrm{Ni}$, and $\mathrm{Fe}$, prepared by a nitriding process of the corresponding oxides using $\mathrm{NH}_{3}$ and a temperature of $350{ }^{\circ} \mathrm{C}$. They reported the catalytic activity order $\mathrm{Co}_{3} \mathrm{Mo}_{3} \mathrm{~N}>\mathrm{Ni}_{3} \mathrm{Mo}_{3} \mathrm{~N}>\mathrm{Fe}_{3} \mathrm{Mo}_{3} \mathrm{~N}>$ $\mathrm{Mo}_{2} \mathrm{~N}$. The same authors verified that the activity of the $\mathrm{Co}_{3} \mathrm{Mo}_{3} \mathrm{~N}$ catalyst increased by adding a small amount of $\mathrm{Cs} ;{ }^{363}$ a $22 \%$ increase in conversion was measured at $450{ }^{\circ} \mathrm{C}$ with respect to the unmodified catalyst. It should be mentioned that 
similar compositions have also been tested, such as $\mathrm{Ni}_{2} \mathrm{Mo}_{3} \mathrm{~N}$, obtaining similar results. $351,350,364$

The compositions presented above have been tested alone or supported on different materials. For example, Liang et al. ${ }^{290}$ compared the catalytic activity of $\mathrm{MoN}_{x}$ with that of $\mathrm{Ni}$ and $\mathrm{NiMoN}_{x}$ bimetallic catalyst supported on alumina. As a reference, they also prepared the $\mathrm{Ni} / \mathrm{MgO}$ catalyst and concluded that the bimetallic catalysts had a higher catalytic activity with respect to the monometallic form and that all the prepared catalysts supported on alumina present a higher conversion than $\mathrm{Ni} / \mathrm{MgO}$. Similarly, a catalyst composed of $\mathrm{Co}-\mathrm{Mo}$ was tested supported on $\mathrm{SiO}_{2}$ and compared with its form promoted with $\mathrm{CaP}$, obtaining an increase in activity until reaching a Ca content of $1 \mathrm{wt} \% .{ }^{365}$ Nevertheless, when Co-Mo was supported directly on $\mathrm{CaP}$, the catalytic activity was minimal. ${ }^{365}$ Lorenzut et al. ${ }^{323}$ prepared several $\mathrm{Fe}$ and $\mathrm{Mo}$ bimetallic catalysts supported on $\mathrm{ZrO}_{2}$ mixed with $\mathrm{Y}_{2} \mathrm{O}_{3}, \mathrm{La}_{2} \mathrm{O}_{3}$, and $\mathrm{CeO}_{2}$, and supported on $\mathrm{La}_{2} \mathrm{O}_{3}-\mathrm{Al}_{2} \mathrm{O}_{3}$ and $\mathrm{Ce}_{0.6} \mathrm{Zr}_{0.4} \mathrm{O}_{2}-$ $\mathrm{Al}_{2} \mathrm{O}_{3}$. They verified the superior activity of the bimetallic catalyst supported on $\mathrm{ZrO}_{2}-\mathrm{Y}_{2} \mathrm{O}_{3}$ that showed a $16 \%$ conversion with respect to a conversion of $6 \%$ for $\mathrm{Fe}$ and $10 \%$ for $\mathrm{Mo}$ at $550{ }^{\circ} \mathrm{C}$. Among the supports tested, $\mathrm{La}_{2} \mathrm{O}_{3}-\mathrm{Al}_{2} \mathrm{O}_{3}$ is the one with the best activity. Catalysts composed of $\mathrm{Co}-\mathrm{Mo}$ have generated a lot of interest in recent times, and have been tested in many types of formulations, for example in its supported form on MCM- $41^{339}$ or $\mathrm{Al}_{2} \mathrm{O}_{3} \cdot{ }^{338,366}$ In both cases, the bimetallic catalysts showed superior catalytic activity with respect to both monometallic compositions. ${ }^{339,338}$

The use of carbon nanofibers (CNFs) as supports was tested by Xie et al. ${ }^{228}$ The nanofiber was combined with a $\mathrm{Co}-\mathrm{Mo}-$ $\mathrm{Fe}-\mathrm{Ni}-\mathrm{Cu}$ alloy and its catalytic activity was compared with that of a Co-Mo bimetallic catalyst and $\mathrm{Ru}$ using the same support. They obtained a TOF 20 times higher than that obtained with $\mathrm{Ru}$, and an even greater increase with respect to the conventional Co-Mo catalyst by varying the amount of Co and Mo in the composition. This multimetallic formulation showed good stability in a $50 \mathrm{~h}$ test at $500{ }^{\circ} \mathrm{C}$. Different ceramic supports have also been used to test the catalytic activity of several bimetallic formulations. For instance, Huang et al. ${ }^{292}$ compared the catalytic activity of bimetallic catalysts composed of $\mathrm{Ni}$ and Co on different oxide supports. The order of catalytic activity of the supports was $\mathrm{Ce}_{0.6} \mathrm{Zr}_{0.3} \mathrm{Y}_{0.1} \mathrm{O}_{2}>\mathrm{CeO}_{2}>\mathrm{Y}_{2} \mathrm{O}_{3}>\mathrm{ZrO}_{2}$. The $\mathrm{Ni}-\mathrm{Co}$ bimetallic catalyst supported on $\mathrm{Ce}_{0.6} \mathrm{Zr}_{0.3} \mathrm{Y}_{0.1} \mathrm{O}_{2}$ showed an increase in ammonia conversion with respect to the monometallic catalysts, a higher TOF, and a great stability in a $72 \mathrm{~h}$ test, demonstrating the synergy between the two metals. Lendzion-Bielun et al. ${ }^{367}$ modified a Fe catalyst promoted with $\mathrm{CaO}, \mathrm{Al}_{2} \mathrm{O}_{3}$, and $\mathrm{K}_{2} \mathrm{O}$ with cobalt, and obtained a higher conversion with respect to the monometallic catalyst. They also tested a catalyst with a $\mathrm{Fe}-\mathrm{Co}$ alloy promoted with $\mathrm{CaO}, \mathrm{Al}_{2} \mathrm{O}_{3}$, and $\mathrm{K}_{2} \mathrm{O},{ }^{368}$ and determined that the catalytic activity depends on the nitriding potential of $\mathrm{Fe}$. In contrast, the addition of chromium or manganese leads to a decrease of the activity of the Co catalyst. ${ }^{337}$ Wang et al. ${ }^{318}$ also tested a Co-Fe bimetallic catalyst and obtained a higher catalytic activity with respect to the monometallic forms of $\mathrm{Co}$ and $\mathrm{Fe}$ (53 vs 25 and $4 \%$ of conversion at $550^{\circ} \mathrm{C}$, respectively). In addition, Zhang et al. ${ }^{322}$ observed that the $\mathrm{Co}-\mathrm{Fe}$ bimetallic catalyst supported on carbon nanotubes is more active than the Fe catalyst on the same support, but it does not exceed the hydrogen production obtained with the Co catalyst. Comparing the catalytic activity of $\mathrm{Mg}-\mathrm{Fe}, \mathrm{Co}-\mathrm{Fe}$ and $\mathrm{Mg}-\mathrm{Co}-\mathrm{Fe}$ bimetallic catalysts, Podila et al. ${ }^{369}$ obtained an activity that follows the order: $\mathrm{Mg}-\mathrm{Co}-\mathrm{Fe}$
$>\mathrm{Mg}-\mathrm{Fe}>\mathrm{Co}-\mathrm{Fe}$. It is interesting to note that in the bimetallic samples the formation of $\mathrm{FeN}_{2}$ was not observed. Following their previous works, Simonsen et al. ${ }^{218}$ synthesized different catalysts composed of $\mathrm{Ni}, \mathrm{Fe}$, and their bimetallic form $\mathrm{Ni}-\mathrm{Fe}$ supported on various materials. In their tests they considered the change in gas composition throughout the reactor, comparing the catalytic performance in the absence of $\mathrm{H}_{2}$ and introducing it into the reagent stream to simulate the conditions in the final part of the reactor. Under the conditions of the first part of the reactor, the bimetallic catalyst supported on alumina showed a much higher activity than the Fe catalyst and activity similar to the $\mathrm{Ni}$ catalyst supported on the same oxide. With the $\mathrm{Ni}-\mathrm{Fe}$ bimetallic catalyst they obtained the same conversion as with a $\mathrm{Ru}$ on alumina catalyst. In the presence of hydrogen, the $\mathrm{Ni}$ catalyst decreases its activity with respect to the bimetallic composition. Regarding the support, they verified that the best catalytic results were obtained with $\mathrm{Al}_{2} \mathrm{O}_{3}$ and $\mathrm{Mg}-\mathrm{Al}$ spinels with respect to $\mathrm{SiO}_{2}, \mathrm{ZrO}_{2}$ and $\mathrm{TiO}_{2}$.

Bimetallic catalysts have not only been tested with the aim of replacing ruthenium or other noble metals, but also to increase their catalytic activity and to be able to reduce their content in the catalyst. In this sense, $\mathrm{McCullough}$ et al. ${ }^{222}$ tested more than 100 bimetallic catalysts based on $\mathrm{Ru}-$ metal-K supported on $\mathrm{Al}_{2} \mathrm{O}_{3}$ through a high-throughput method for the decomposition of ammonia at low temperature. For each catalyst they tested three different metal loadings $(\mathrm{Ru} / \mathrm{Metal}=3: 1,2: 2$, and $1: 3$ on a weight basis). They concluded that the bimetallic catalysts that have a higher activity with respect to the K-promoted Ru catalyst follow the order of activity $\mathrm{Ir} \approx \mathrm{Sc} \approx \mathrm{Zr} \approx \mathrm{Ta}>\mathrm{Sr} \approx \mathrm{Y}>\mathrm{Ca}>\mathrm{Mg}$ $>$ Hf. The K-promoted $\mathrm{Ru}-\mathrm{Sr}$ and $\mathrm{Ru}-\mathrm{Fe}$ catalysts supported on $\mathrm{Al}_{2} \mathrm{O}_{3}$ showed higher TOF values than the K-promoted $\mathrm{Ru}$ / $\mathrm{Al}_{2} \mathrm{O}_{3}$ catalyst $\left(1.8,1.0\right.$, and $0.3 \mathrm{~s}^{-1}$ at $400{ }^{\circ} \mathrm{C}$, respectively, using pure ammonia). Ruthenium bimetallic catalysts have not only been tested to increase catalytic activity, but also to increase stability. Thus, Chen et al. ${ }^{231}$ prepared a $\mathrm{Ru}-\mathrm{Fe}$ catalyst supported on CNTs obtaining a slightly lower catalytic activity than the monometallic $\mathrm{Ru} / \mathrm{CNT}$ s catalyst, but a superior longterm stability $(60 \mathrm{~h})$.

Varisli and Rona ${ }^{243}$ compared the $\mathrm{H}_{2}$ production of $\mathrm{Pt}-\mathrm{Ni}$ and $\mathrm{Pt}-\mathrm{Sn}$ catalysts supported on $\mathrm{SiO}_{2}$ and MCM-41.They reported a higher activity of the monometallic Pt/MCM-41 compared to the bimetallic forms, and that $\mathrm{Pt}-\mathrm{Ni} / \mathrm{MCM}-41$ presents a very low ammonia conversion. In contrast, the bimetallic catalyst supported on $\mathrm{SiO}_{2}$ had a slightly higher activity compared to the monometallic catalyst. Chellappa et al. ${ }^{370}$ also estimated the apparent activation energy for a Ni-Pt bimetallic catalyst supported on alumina and found that it is very similar to that obtained for nickel, concluding that platinum behaves more like a stabilizer to prevent sintering of nickel active sites instead of increasing catalytic activity.

A catalyst composed of $\mathrm{Ni}-\mathrm{Pd}^{241}$ exhibited superior catalytic activity compared to monometallic catalysts. The bimetallic catalyst demonstrated the synergistic effect of $\mathrm{Pd}$ and $\mathrm{Ni}$ for the decomposition of ammonia. A bimetallic catalyst composed of Ir and $\mathrm{Ni}$ supported on alumina also presented good catalytic results, with a $12 \%$ higher conversion at $500{ }^{\circ} \mathrm{C}$ with respect to the monometallic $\mathrm{Ni} / \mathrm{Al}_{2} \mathrm{O}_{3}$ catalyst under the same reaction conditions. ${ }^{291}$ Similarly, Dasireddy and Likozar ${ }^{250}$ prepared a bimetallic catalyst composed of $\mathrm{Cu}$ and $\mathrm{Zn}$ supported on alumina, demonstrating its superior catalytic activity with respect to the corresponding monometallic catalysts (78 vs 61 and $15 \%$ at $500{ }^{\circ} \mathrm{C}$, respectively). The same authors also improved the activity and stability of the $\mathrm{Cu}-\mathrm{Zn} / \mathrm{Al}_{2} \mathrm{O}_{3}$ catalyst 
Table 9. Catalysts with Bimetallic and Multimetallic Active Phase Used to Decompose Ammonia and Their Catalytic Performances at $1 \mathrm{~atm}$

\begin{tabular}{|c|c|c|c|c|c|c|c|c|c|c|c|c|}
\hline active phase & wt $\%$ & support & promoter & $\begin{array}{c}\text { WHSV } \\
\left(\mathrm{mL} \mathrm{g}^{-1} \mathrm{~h}^{-1}\right)\end{array}$ & $\begin{array}{c}\text { GHSV } \\
\left(\mathrm{h}^{-1}\right)\end{array}$ & $\begin{array}{c}\% \mathrm{NH}_{3} \\
\text { inlet flow }\end{array}$ & $\begin{array}{c}T\left(^{\circ}\right. \\
\mathrm{C})\end{array}$ & $\begin{array}{c}\text { conv } \\
(\%)\end{array}$ & $\begin{array}{c}\mathrm{H}_{2} \text { rate } \\
\left(\mathrm{mmol} \mathrm{g}^{-1} \mathrm{~s}^{-1}\right)\end{array}$ & $\begin{array}{c}E_{\mathrm{a}} \\
\left(\mathrm{kJ} \mathrm{mol}^{-1}\right)\end{array}$ & $\begin{array}{l}\text { TOF } \\
\left(s^{-1}\right)\end{array}$ & ref \\
\hline $\mathrm{Co}_{3} \mathrm{Mo}_{3} \mathrm{~N}$ & & & & & 6000 & 100 & 500 & 39 & & 93 & & 347 \\
\hline $\mathrm{Co}_{3} \mathrm{Mo}_{3} \mathrm{~N}$ & & & & 6000 & & 100 & 550 & 97 & & 60 & & 348 \\
\hline $\mathrm{Co}_{3} \mathrm{Mo}_{3} \mathrm{~N}$ & & & & 6000 & & 100 & 550 & 94 & & 70 & & 350 \\
\hline $\mathrm{Co}_{3} \mathrm{Mo}_{3} \mathrm{~N}$ & & & & 6000 & & 100 & 450 & 26 & & 86 & 0.7 & 363 \\
\hline $\mathrm{Co}_{3} \mathrm{Mo}_{3} \mathrm{~N}$ & & & Cs & 6000 & & 100 & 450 & 48 & & 71 & 1.3 & 363 \\
\hline $\mathrm{Co}-\mathrm{Cr}$ & & & $\mathrm{Al}_{2} \mathrm{O}_{3}, \mathrm{CaO}, \mathrm{K}_{2} \mathrm{O}$ & 24000 & & 100 & 500 & 35 & 0.12 & & & 337 \\
\hline $\mathrm{Co}-\mathrm{Fe}$ & & & & & & 15 & 550 & 53 & & & & 318 \\
\hline $\mathrm{Co}-\mathrm{Fe}$ & & & & & 6000 & 100 & 550 & 77 & & 63 & & 369 \\
\hline $\mathrm{Co}-\mathrm{Fe}$ & & & $\mathrm{CaO}, \mathrm{Al}_{2} \mathrm{O}_{3}, \mathrm{~K}_{2} \mathrm{O}$ & 24000 & & 100 & 500 & 13 & & & & 368 \\
\hline $\mathrm{Co}-\mathrm{Fe}$ & 5 & CNTs & & 36000 & & 100 & 550 & & 0.17 & 110 & 5.4 & 322 \\
\hline $\mathrm{Co}-\mathrm{Mn}$ & & & $\mathrm{Al}_{2} \mathrm{O}_{3}, \mathrm{CaO}, \mathrm{K}_{2} \mathrm{O}$ & 24000 & & 100 & 500 & 23 & 0.10 & & & 337 \\
\hline $\mathrm{Co}-\mathrm{Mo}$ & & & & 36000 & & 100 & 550 & 37 & & & & 361 \\
\hline $\mathrm{Co}-\mathrm{Mo}$ & 5 & $\mathrm{Al}_{2} \mathrm{O}_{3}$ & & 36000 & & 100 & 500 & 55 & & & & 338 \\
\hline $\mathrm{Co}-\mathrm{Mo}$ & $5 / 8$ & $\mathrm{Al}_{2} \mathrm{O}_{3}$ & & 30000 & & 5 & 527 & 60 & & 106 & & 366 \\
\hline $\mathrm{Co}-\mathrm{Mo}$ & $2.5 / 2.5$ & $\mathrm{CaP}$ & & 6000 & & 100 & 500 & 10 & & & & 365 \\
\hline $\mathrm{Co}-\mathrm{Mo}$ & 10 & CNFs & & 36000 & & 100 & 500 & 5 & & & 0.06 & 228 \\
\hline $\mathrm{Co}-\mathrm{Mo}$ & 5 & MCM-41 & & 36000 & & 100 & 500 & 52 & & & & 339 \\
\hline $\mathrm{Co}-\mathrm{Mo}$ & $2.5 / 2.5$ & $\mathrm{SiO}_{2}$ & & 6000 & & 100 & 500 & 20 & & 82 & & 365 \\
\hline $\mathrm{Co}-\mathrm{Mo}$ & 5 & $\mathrm{SiO}_{2}$ & & 36000 & & 100 & 500 & 15 & & & & 339 \\
\hline $\mathrm{Co}-\mathrm{Mo}$ & $2.5 / 2.5$ & $\mathrm{SiO}_{2}$ & $\mathrm{CaP}$ & 6000 & & 100 & 500 & 24 & & 76 & & 365 \\
\hline $\begin{array}{l}\mathrm{Co}-\mathrm{Mo}- \\
\mathrm{Fe}-\mathrm{Ni}-\mathrm{Cu}\end{array}$ & 9.3 & CNFs & & 36000 & & 100 & 500 & 100 & & 123 & 7.0 & 228 \\
\hline $\mathrm{CoMoN}_{x}$ & $10 / 2$ & CNTs & & & 11000 & 10 & 550 & 50 & & & & 362 \\
\hline $\mathrm{CoMoN}_{x}$ & $10 / 2$ & CNTs & $\mathrm{Ba}$ & & 11000 & 10 & 550 & 67 & & & & 362 \\
\hline $\mathrm{CoMoN}_{x}$ & $10 / 2$ & CNTs & $\mathrm{Ce}$ & & 11000 & 10 & 550 & 50 & & & & 362 \\
\hline $\mathrm{CoMoN}_{x}$ & $10 / 2$ & CNTs & $\mathrm{K}$ & & 11000 & 10 & 550 & 56 & & & & 362 \\
\hline $\mathrm{CoMoN}_{x}$ & $10 / 2$ & CNTs & $\mathrm{La}$ & & 11000 & 10 & 550 & 83 & & & & 362 \\
\hline $\mathrm{CoMoN}_{x}$ & $10 / 2$ & CNTs & $\mathrm{Zr}$ & & 11000 & 10 & 550 & 50 & & & & 362 \\
\hline $\mathrm{Cu}-\mathrm{Zn}$ & $50 / 30$ & $\mathrm{Al}_{2} \mathrm{O}_{3}$ & & 60000 & & 9.8 & 500 & 78 & 0.60 & 56 & & 250 \\
\hline $\mathrm{Fe}_{3} \mathrm{Mo}_{3} \mathrm{~N}$ & & & & 6000 & & 100 & 550 & 78 & & 73 & & 349 \\
\hline $\mathrm{Fe}_{3} \mathrm{Mo}_{3} \mathrm{~N}$ & & & & 6000 & & 100 & 550 & 75 & & 89 & & 350 \\
\hline $\mathrm{Fe}-\mathrm{Mo}$ & $5 / 5$ & $\begin{array}{l}\mathrm{Ce}_{0.6} \mathrm{Zr}_{0.4} \mathrm{O}_{2}- \\
\mathrm{Al}_{2} \mathrm{O}_{3}\end{array}$ & & 46000 & & 100 & 550 & 17 & & & & 323 \\
\hline $\mathrm{Fe}-\mathrm{Mo}$ & $5 / 5$ & $\mathrm{CeO}_{2}-\mathrm{ZrO}_{2}$ & & 46000 & & 100 & 550 & 16 & & & & 323 \\
\hline $\mathrm{Fe}-\mathrm{Mo}$ & $5 / 5$ & $\mathrm{La}_{2} \mathrm{O}_{3}-\mathrm{Al}_{2} \mathrm{O}_{3}$ & & 46000 & & 100 & 550 & 50 & & & & 323 \\
\hline $\mathrm{Fe}-\mathrm{Mo}$ & $5 / 5$ & $\mathrm{La}_{2} \mathrm{O}_{3}-\mathrm{ZrO}_{2}$ & & 46000 & & 100 & 550 & 16 & & & & 323 \\
\hline $\mathrm{Fe}-\mathrm{Mo}$ & $5 / 5$ & $\mathrm{Y}_{2} \mathrm{O}_{3}-\mathrm{ZrO}_{2}$ & & 46000 & & 100 & 550 & 16 & & & & 323 \\
\hline $\mathrm{Li}_{3} \mathrm{FeN}_{2}$ & & & & 60000 & & 100 & 500 & 31 & & & & 359 \\
\hline $\mathrm{Mg}-\mathrm{Co}-\mathrm{Fe}$ & & & & & 6000 & 100 & 550 & 99 & & 45 & & 369 \\
\hline $\mathrm{Mg}-\mathrm{Fe}$ & & & & & 6000 & 100 & 550 & 86 & & 59 & & 369 \\
\hline $\mathrm{Ni}_{2} \mathrm{Mo}_{3} \mathrm{~N}$ & & & & & 6000 & 100 & 500 & 87 & & 66 & & 351 \\
\hline $\mathrm{Ni}_{3} \mathrm{Mo}_{3} \mathrm{~N}$ & & & & 6000 & & 100 & 550 & 83 & & 84 & & 350 \\
\hline $\mathrm{Ni}-\mathrm{Co}$ & $1-9$ & $\mathrm{Ce}_{0.6} \mathrm{Zr}_{0.3} \mathrm{Y}_{0.1} \mathrm{O}_{2}$ & & 6000 & & 100 & 350 & 10 & 0.012 & 41 & 0.68 & 292 \\
\hline $\mathrm{Ni}-\mathrm{Fe}$ & $2 / 8$ & $\mathrm{Al}_{2} \mathrm{O}_{3}$ & & 240000 & 80000 & 50 & 500 & 98 & & & 0.037 & 218 \\
\hline $\mathrm{Ni}-\mathrm{Fe}$ & $2 / 8$ & $\mathrm{Mg}-\mathrm{Al}$ spinel & & 240000 & 80000 & 50 & 500 & 98 & & & & 218 \\
\hline $\mathrm{Ni}-\mathrm{Fe}$ & $2 / 8$ & $\mathrm{SiO}_{2}$ & & 240000 & 80000 & 50 & 500 & 35 & & & & 218 \\
\hline $\mathrm{Ni}-\mathrm{Fe}$ & $2 / 8$ & $\mathrm{TiO}_{2}$ & & 240000 & 80000 & 50 & 500 & 9 & & & & 218 \\
\hline $\mathrm{Ni}-\mathrm{Fe}$ & $2 / 8$ & $\mathrm{ZrO}_{2}$ & & 240000 & 80000 & 50 & 500 & 60 & & & & 218 \\
\hline $\mathrm{Ni}-\mathrm{Ir}$ & $10 / 0.7$ & $\mathrm{Al}_{2} \mathrm{O}_{3}$ & & 9500 & & 5.9 & 400 & 44 & & & & 291 \\
\hline $\mathrm{NiMoN}_{x}$ & & & & & 21600 & 100 & 500 & 29 & & & & 364 \\
\hline $\mathrm{NiMoN}_{x}$ & $6 / 10$ & $\mathrm{Al}_{2} \mathrm{O}_{3}$ & & & 1800 & 100 & 650 & 100 & & & & 290 \\
\hline $\mathrm{Pd}-\mathrm{Ni}$ & & & & & 20600 & 100 & 450 & 50 & & 64 & & 241 \\
\hline $\mathrm{Pd}-\mathrm{Ni}$ & & & $\mathrm{Ca}$ & & 20600 & 100 & 450 & 85 & & 59 & & 241 \\
\hline $\mathrm{Pt}-\mathrm{Ni}$ & & $\mathrm{Al}_{2} \mathrm{O}_{3}$ & & & & 100 & 540 & 97 & & 47 & & 370 \\
\hline $\mathrm{Pt}-\mathrm{Ni}$ & & MCM-41 & & 5100 & & 100 & 500 & & 0.009 & & & 243 \\
\hline $\mathrm{Pt}-\mathrm{Ni}$ & & $\mathrm{SiO}_{2}$ & & 5100 & & 100 & 500 & & 0.086 & & & 243 \\
\hline $\mathrm{Pt}-\mathrm{Sn}$ & & MCM-41 & & 5100 & & 100 & 500 & & 0.103 & & & 243 \\
\hline $\mathrm{Pt}-\mathrm{Sn}$ & & $\mathrm{SiO}_{2}$ & & 5100 & & 100 & 500 & & 0.089 & & & 243 \\
\hline $\mathrm{Ru}-\mathrm{Ag}$ & $3 / 1$ & $\mathrm{Al}_{2} \mathrm{O}_{3}$ & $\mathrm{~K}$ & 30000 & & 1 & 300 & 64 & & & & 222 \\
\hline $\mathrm{Ru}-\mathrm{Au}$ & $3 / 1$ & $\mathrm{Al}_{2} \mathrm{O}_{3}$ & $\mathrm{~K}$ & 30000 & & 1 & 300 & 58 & & & & 222 \\
\hline $\mathrm{Ru}-\mathrm{Bi}$ & $3 / 1$ & $\mathrm{Al}_{2} \mathrm{O}_{3}$ & $\mathrm{~K}$ & 30000 & & 1 & 300 & 23 & & & & 222 \\
\hline
\end{tabular}


Table 9. continued

\begin{tabular}{|c|c|c|c|c|c|c|c|c|c|c|c|c|}
\hline active phase & wt $\%$ & support & promoter & $\begin{array}{c}\text { WHSV } \\
\left(\mathrm{mL} \mathrm{g}^{-1} \mathrm{~h}^{-1}\right)\end{array}$ & $\begin{array}{c}\text { GHSV } \\
\left(\mathrm{h}^{-1}\right)\end{array}$ & $\begin{array}{c}\% \mathrm{NH}_{3} \\
\text { inlet flow }\end{array}$ & $\begin{array}{c}T\left(^{\circ}\right. \\
\mathrm{C})\end{array}$ & $\begin{array}{l}\text { conv } \\
(\%)\end{array}$ & $\begin{array}{c}\mathrm{H}_{2} \text { rate } \\
\left(\mathrm{mmol} \mathrm{g}^{-1} \mathrm{~s}^{-1}\right)\end{array}$ & $\begin{array}{c}E_{\mathrm{a}} \\
\left(\mathrm{kJ} \mathrm{mol}^{-1}\right)\end{array}$ & $\begin{array}{l}\text { TOF } \\
\left(\mathrm{s}^{-1}\right)\end{array}$ & ref \\
\hline $\mathrm{Ru}-\mathrm{Ca}$ & $3 / 1$ & $\mathrm{Al}_{2} \mathrm{O}_{3}$ & $\mathrm{~K}$ & 30000 & & 1 & 300 & 91 & & & & 222 \\
\hline $\mathrm{Ru}-\mathrm{Cd}$ & $2 / 2$ & $\mathrm{Al}_{2} \mathrm{O}_{3}$ & $\mathrm{~K}$ & 30000 & & 1 & 300 & 29 & & & & 222 \\
\hline $\mathrm{Ru}-\mathrm{Co}$ & $3 / 1$ & $\mathrm{Al}_{2} \mathrm{O}_{3}$ & $\mathrm{~K}$ & 30000 & & 1 & 300 & 43 & & & & 222 \\
\hline $\mathrm{Ru}-\mathrm{Cr}$ & $3 / 1$ & $\mathrm{Al}_{2} \mathrm{O}_{3}$ & $\mathrm{~K}$ & 30000 & & 1 & 300 & 56 & & & & 222 \\
\hline $\mathrm{Ru}-\mathrm{Cu}$ & $3 / 1$ & $\mathrm{Al}_{2} \mathrm{O}_{3}$ & $\mathrm{~K}$ & 30000 & & 1 & 300 & 30 & & & & 222 \\
\hline $\mathrm{Ru}-\mathrm{Fe}$ & $2 / 2$ & $\mathrm{Al}_{2} \mathrm{O}_{3}$ & $\mathrm{~K}$ & 30000 & & 1 & 300 & 39 & & & & 222 \\
\hline $\mathrm{Ru}-\mathrm{Fe}$ & $3 / 1$ & $\mathrm{Al}_{2} \mathrm{O}_{3}$ & $\mathrm{~K}$ & 5400 & & 100 & 400 & 44 & & 248 & 0.97 & 222 \\
\hline $\mathrm{Ru}-\mathrm{Fe}$ & 1.7 & CNTs & & 6000 & & 100 & 450 & 85 & & & & 231 \\
\hline $\mathrm{Ru}-\mathrm{Hf}$ & $1 / 3$ & $\mathrm{Al}_{2} \mathrm{O}_{3}$ & $\mathrm{~K}$ & 30000 & & 1 & 300 & 82 & & & & 222 \\
\hline $\mathrm{Ru}-\mathrm{In}$ & $3 / 1$ & $\mathrm{Al}_{2} \mathrm{O}_{3}$ & K & 30000 & & 1 & 300 & 24 & & & & 222 \\
\hline $\mathrm{Ru}-\mathrm{Ir}$ & $3 / 1$ & $\mathrm{Al}_{2} \mathrm{O}_{3}$ & $\mathrm{~K}$ & 30000 & & 1 & 300 & 100 & & & & 222 \\
\hline $\mathrm{Ru}-\mathrm{Mg}$ & $3 / 1$ & $\mathrm{Al}_{2} \mathrm{O}_{3}$ & $\mathrm{~K}$ & 30000 & & 1 & 300 & 90 & & & & 222 \\
\hline $\mathrm{Ru}-\mathrm{Mn}$ & $2 / 2$ & $\mathrm{Al}_{2} \mathrm{O}_{3}$ & K & 30000 & & 1 & 300 & 47 & & & & 222 \\
\hline $\mathrm{Ru}-\mathrm{Mo}$ & $3 / 1$ & $\mathrm{Al}_{2} \mathrm{O}_{3}$ & K & 30000 & & 1 & 300 & 18 & & & & 222 \\
\hline $\mathrm{Ru}-\mathrm{Nb}$ & $1 / 3$ & $\mathrm{Al}_{2} \mathrm{O}_{3}$ & K & 30000 & & 1 & 300 & 17 & & & & 222 \\
\hline $\mathrm{Ru}-\mathrm{Ni}$ & $3 / 1$ & $\mathrm{Al}_{2} \mathrm{O}_{3}$ & $\mathrm{~K}$ & 30000 & & 1 & 300 & 55 & & & & 222 \\
\hline $\mathrm{Ru}-\mathrm{Ni}$ & $2.5 / 0.5$ & $\mathrm{CeO}_{2}$ & & 15000 & 3660 & 55 & 400 & 50 & & 107 & 2.0 & 372 \\
\hline $\mathrm{Ru}-\mathrm{Os}$ & $1 / 3$ & $\mathrm{Al}_{2} \mathrm{O}_{3}$ & K & 30000 & & 1 & 300 & 34 & & & & 222 \\
\hline $\mathrm{Ru}-\mathrm{Pb}$ & $1 / 3$ & $\mathrm{Al}_{2} \mathrm{O}_{3}$ & K & 30000 & & 1 & 300 & 12 & & & & 222 \\
\hline $\mathrm{Ru}-\mathrm{Pd}$ & $3 / 1$ & $\mathrm{Al}_{2} \mathrm{O}_{3}$ & K & 30000 & & 1 & 300 & 62 & & & & 222 \\
\hline $\mathrm{Ru}-\mathrm{Pt}$ & $3 / 1$ & $\mathrm{Al}_{2} \mathrm{O}_{3}$ & K & 30000 & & 1 & 300 & 28 & & & & 222 \\
\hline $\mathrm{Ru}-\mathrm{Re}$ & $2 / 2$ & $\mathrm{Al}_{2} \mathrm{O}_{3}$ & K & 30000 & & 1 & 300 & 10 & & & & 222 \\
\hline $\mathrm{Ru}-\mathrm{Rh}$ & $2 / 2$ & $\mathrm{Al}_{2} \mathrm{O}_{3}$ & K & 30000 & & 1 & 300 & 79 & & & & 222 \\
\hline $\mathrm{Ru}-\mathrm{Sc}$ & $3 / 1$ & $\mathrm{Al}_{2} \mathrm{O}_{3}$ & $\mathrm{~K}$ & 30000 & & 1 & 300 & 100 & & & & 222 \\
\hline $\mathrm{Ru}-\mathrm{Sn}$ & $3 / 1$ & $\mathrm{Al}_{2} \mathrm{O}_{3}$ & $\mathrm{~K}$ & 30000 & & 1 & 300 & 20 & & & & 222 \\
\hline $\mathrm{Ru}-\mathrm{Sr}$ & $1 / 3$ & $\mathrm{Al}_{2} \mathrm{O}_{3}$ & $\mathrm{~K}$ & 30000 & & 1 & 300 & 93 & & & & 222 \\
\hline $\mathrm{Ru}-\mathrm{Sr}$ & $1 / 3$ & $\mathrm{Al}_{2} \mathrm{O}_{3}$ & $\mathrm{~K}$ & 5400 & & 100 & 400 & 80 & & 156 & 1.78 & 222 \\
\hline $\mathrm{Ru}-\mathrm{Ta}$ & $3 / 1$ & $\mathrm{Al}_{2} \mathrm{O}_{3}$ & $\mathrm{~K}$ & 30000 & & 1 & 300 & 100 & & & & 222 \\
\hline $\mathrm{Ru}-\mathrm{W}$ & $3 / 1$ & $\mathrm{Al}_{2} \mathrm{O}_{3}$ & $\mathrm{~K}$ & 30000 & & 1 & 300 & 57 & & & & 222 \\
\hline $\mathrm{Ru}-\mathrm{Y}$ & $2 / 2$ & $\mathrm{Al}_{2} \mathrm{O}_{3}$ & $\mathrm{~K}$ & 30000 & & 1 & 300 & 93 & & & & 222 \\
\hline $\mathrm{Ru}-\mathrm{Zn}$ & $3 / 1$ & $\mathrm{Al}_{2} \mathrm{O}_{3}$ & K & 30000 & & 1 & 300 & 49 & & & & 222 \\
\hline $\mathrm{Ru}-\mathrm{Zr}$ & $3 / 1$ & $\mathrm{Al}_{2} \mathrm{O}_{3}$ & $\mathrm{~K}$ & 30000 & & 1 & 300 & 100 & & & & 222 \\
\hline
\end{tabular}

by varying the composition and the preparation method. ${ }^{371}$ Finally, the superior stability of a bimetallic catalyst composed of $\mathrm{Ni}$ and $\mathrm{Ru}$ supported on ceria compared to $\mathrm{Ru} / \mathrm{CeO}_{2}$ and its superior activity compared to $\mathrm{Ni} / \mathrm{CeO}_{2}$ has recently been demonstrated. ${ }^{219,372}$ Catalysts with bimetallic and multimetallic active phases used to decompose ammonia and their catalytic performances are reported in Table 9.

3.1.5. Metal Amides and Imides. The alkali metal amides, such as $\mathrm{LiNH}_{2}, \mathrm{KNH}_{2}$, and $\mathrm{NaNH}_{2}$, have proven to be promising materials in the decomposition of ammonia, offering comparable or better conversion results with respect to catalysts composed of ruthenium. For instance, under the same catalytic conditions, lithium amide showed a much higher activity compared to the $\mathrm{Ru} / \mathrm{Al}_{2} \mathrm{O}_{3}$ and $\mathrm{Ni} / \mathrm{SiO}_{2}-\mathrm{Al}_{2} \mathrm{O}_{3}$ catalysts (91 vs 54 and $34 \%$ at $500{ }^{\circ} \mathrm{C}$, respectively). ${ }^{32}$ Using the amide $\mathrm{NaNH}_{2}$, a conversion equivalent to that of $\mathrm{Ru} / \mathrm{Al}_{2} \mathrm{O}_{3}$ was obtained..$^{373}$ Moreover, $\mathrm{NaNH}_{2}$ showed higher conversion than $\mathrm{LiNH}_{2}$ at temperatures below ca. $420{ }^{\circ} \mathrm{C}$, while at higher temperature the two amides presented a similar activity. ${ }^{32}$

It was determined that the active phase of catalysts composed of amides varies according to the reaction conditions and the alkali metal chosen. In the case of $\mathrm{Li}$ amides, the active phase is the imide form, $-\mathrm{NH} .{ }^{32}$ Makepeace et al. ${ }^{374}$ prepared lithium imide, modified it with $\mathrm{Ca}$ and $\mathrm{Mg}$, and compared the results with a lithium amide-imide mixture. They observed that the modified forms show a higher conversion at low temperatures with respect to the unmodified catalyst ( 48 and 40 vs $33 \%$ at 440 ${ }^{\circ} \mathrm{C}$, respectively), and that $\mathrm{Li}_{2} \mathrm{Ca}(\mathrm{NH})_{2}$ presents a higher activity with respect to $\mathrm{Li}_{2} \mathrm{Mg}(\mathrm{NH})_{2}$. Moreover, Wood and Makepeace also analyzed the compatibility of the lithium amide-imide catalyst with various support materials: activated carbon, silicon dioxide, aluminum oxide, and magnesium oxide. $^{375}$ However, for all configurations there was no improvement in the catalytic performance compared to the unsupported lithium amide-imide. Carbon is not a suitable support despite its high surface area since it reacts with the catalyst to form the lithium carbodiimide species, $\mathrm{Li}_{2} \mathrm{NCN}$. Similarly, silica and alumina are also not good supports because they react with the catalyst and form lithium oxides. In contrast, $\mathrm{MgO}$ shows better synergy with amide-imide, allowing the formation of a ternary nitride instead of a ternary oxide, which is active for the decomposition of ammonia. However, there is no consensus in the literature about the role of ternary nitrides in the decomposition of ammonia. ${ }^{376,377}$ Bramwell et al. ${ }^{224}$ compared the catalytic activity of $\mathrm{LiNH}_{2}$ alone or supported on carbon. In the case of the supported catalyst, no activity was recorded due to the formation of $\mathrm{Li}_{2} \mathrm{NCN}$. However, by adding $\mathrm{Ni}$ to the supported catalyst, the reaction can be retarded to 450 ${ }^{\circ} \mathrm{C}$. Therefore, the $\mathrm{LiNH}_{2} / \mathrm{Ni} / \mathrm{C}$ catalyst showed higher catalytic activity with respect to $\mathrm{LiNH}_{2}$ (53 vs $13 \%$ at 400 ${ }^{\circ} \mathrm{C}$ ). When these results are compared with those obtained using a $\mathrm{Ru}$ catalyst supported on alumina, the latter allows a $26 \%$ higher conversion to $\mathrm{LiNH}_{2} / \mathrm{Ni} / \mathrm{C}$ at $400{ }^{\circ} \mathrm{C}$ under the reaction conditions analyzed.

Amides and imides have been tested alone or in combination with other compounds. For example, Chang et al. ${ }^{378}$ prepared 
Table 10. Catalysts with Metal Amides and Imides As Active Phase Used to Decompose Ammonia and Their Catalytic Performances at 1 atm

\begin{tabular}{|c|c|c|c|c|c|c|c|c|c|c|c|c|}
\hline active phase & wt $\%$ & support & promoter & $\begin{array}{c}\text { WHSV } \\
\left(\mathrm{mL} \mathrm{g}^{-1} \mathrm{~h}^{-1}\right)\end{array}$ & $\begin{array}{c}\text { GHSV } \\
\left(\mathrm{h}^{-1}\right)\end{array}$ & $\begin{array}{c}\% \mathrm{NH}_{3} \\
\text { inlet flow }\end{array}$ & $\begin{array}{c}\mathrm{T} \\
\left({ }^{\circ} \mathrm{C}\right)\end{array}$ & $\begin{array}{c}\text { conv } \\
(\%)\end{array}$ & $\begin{array}{c}\mathrm{H}_{2} \text { rate } \\
\left(\mathrm{mmol} \mathrm{g}^{-1} \mathrm{~s}^{-1}\right)\end{array}$ & $\begin{array}{c}E_{\mathrm{a}} \\
\left(\mathrm{kJ} \mathrm{mol}^{-1}\right)\end{array}$ & $\begin{array}{l}\text { TOF } \\
\left(s^{-1}\right)\end{array}$ & ref \\
\hline $\mathrm{K}_{2}\left[\mathrm{Mn}\left(\mathrm{NH}_{2}\right)_{4}\right]$ & & & & 60000 & & 5 & 500 & 48 & & & & 360 \\
\hline $\mathrm{Li}_{2} \mathrm{Ca}(\mathrm{NH})_{2}$ & & & & 7200 & & 100 & 440 & 48 & & 120 & & 374 \\
\hline $\mathrm{Li}_{2} \mathrm{Mg}(\mathrm{NH})_{2}$ & & & & 7200 & & 100 & 440 & 40 & & 112 & & 374 \\
\hline $\mathrm{Li}_{2} \mathrm{NH}$ & & & & 60000 & & 5 & 400 & 0 & & 150 & & 232 \\
\hline $\mathrm{Li}_{2} \mathrm{NH}$ & & & & 60000 & & 100 & 500 & 26 & & & & 233 \\
\hline $\mathrm{Li}_{2} \mathrm{NH}-\mathrm{Co}$ & & & & & & & & & & & & 233 \\
\hline $\mathrm{Li}_{2} \mathrm{NH}-\mathrm{CrN}$ & & & & & & & & & & & & 233 \\
\hline $\mathrm{Li}_{2} \mathrm{NH}-\mathrm{Cu}$ & & & & & & & & & & & & 233 \\
\hline $\mathrm{Li}_{2} \mathrm{NH}-\mathrm{Fe}_{2} \mathrm{~N}$ & & & & & & & & & & 50 & & 233 \\
\hline $\mathrm{Li}_{2} \mathrm{NH}-\mathrm{MnN}$ & & & & & & & & & & & & 233 \\
\hline $\mathrm{Li}_{2} \mathrm{NH}-\mathrm{Ni}$ & & & & & & & & & & & & 233 \\
\hline $\mathrm{Li}_{2} \mathrm{NH}-\mathrm{TiN}$ & & & & & & & & & & & & 233 \\
\hline $\mathrm{Li}_{2} \mathrm{NH}-\mathrm{VN}$ & & & & & & & & & & & & 233 \\
\hline $\mathrm{Li}_{2-\mathrm{x}} \mathrm{NH}_{1+\mathrm{x}}$ & & & & 7200 & & 100 & 440 & 33 & & 120 & & 374 \\
\hline $\mathrm{LiNH}_{2}$ & & & & 7200 & & 100 & 450 & 91 & & & 0.002 & 32 \\
\hline $\mathrm{LiNH}_{2}$ & & & & & 13000 & 10 & 400 & 13 & & & & 224 \\
\hline $\mathrm{LiNH}_{2}$ & 50 & $\begin{array}{l}\text { activated } \\
\text { charcoal }\end{array}$ & & 1800 & & 100 & 475 & 23 & & & & 375 \\
\hline $\mathrm{LiNH}_{2}$ & 50 & $\mathrm{Al}_{2} \mathrm{O}_{3}$ & & 1800 & & 100 & 475 & 60 & & & & 375 \\
\hline $\mathrm{LiNH}_{2}$ & 27 & $\mathrm{C}$ & & & 13000 & 10 & 400 & 0 & & & & 224 \\
\hline $\mathrm{LiNH}_{2}$ & 50 & $\mathrm{MgO}$ & & 1800 & & 100 & 475 & 98 & & & & 375 \\
\hline $\mathrm{LiNH}_{2}$ & 50 & $\mathrm{SiO}_{2}$ & & 1800 & & 100 & 475 & 88 & & & & 375 \\
\hline $\mathrm{Mn}_{6} \mathrm{~N}_{5}-\mathrm{CaNH}$ & & & & 36000 & & 100 & 450 & 20 & & 86 & & 380 \\
\hline $\mathrm{MnN}-\mathrm{KNH}_{2}$ & 39.1 & & & 13500 & & 100 & 465 & 21 & & 88 & 1.2 & 378 \\
\hline $\mathrm{MnN}-\mathrm{Li}_{2} \mathrm{NH}$ & & & & 20000 & & 100 & 550 & 90 & & 75 & & 379 \\
\hline $\mathrm{MnN}_{-} \mathrm{LiNH}_{2}$ & 49.9 & & & 13500 & & 100 & 465 & 58 & & 80 & 2.0 & 378 \\
\hline $\mathrm{MnN}_{-} \mathrm{NaNH}_{2}$ & 46.7 & & & 13500 & & 100 & 465 & 20 & & 89 & 0.9 & 378 \\
\hline $\mathrm{NaNH}_{2}$ & & & & 7200 & & 100 & 450 & 55 & & & 0.002 & 32 \\
\hline $\mathrm{NaNH}_{2}$ & & & & 60000 & & 100 & 500 & 20 & & & & 233 \\
\hline $\mathrm{Ni}-\mathrm{KNH}_{2}$ & $8 / 20$ & GNP & & 15000 & 12000 & 10 & 375 & 44 & & 63 & & 235 \\
\hline $\mathrm{Ni}-\mathrm{LiNH}_{2}$ & 27 & $\mathrm{C}$ & & & 13000 & 10 & 400 & 53 & & & & 224 \\
\hline $\mathrm{Ni}-\mathrm{NaNH}_{2}$ & $8 / 20$ & GNP & & 15000 & 12000 & 10 & 375 & 33 & & 62 & & 235 \\
\hline $\mathrm{Rb}_{2}\left[\mathrm{Mn}\left(\mathrm{NH}_{2}\right)_{4}\right]$ & & & & 60000 & & 5 & 500 & 68 & & & & 360 \\
\hline $\mathrm{Ru}-\mathrm{Ba}\left(\mathrm{NH}_{2}\right)_{2}$ & 4.4 & & & 60000 & & 100 & 400 & 20 & 0.13 & 73 & 1.29 & 237 \\
\hline $\mathrm{Ru}-\mathrm{Ca}\left(\mathrm{NH}_{2}\right)_{2}$ & 4.6 & & & 60000 & & 100 & 400 & 8 & 0.08 & 77 & 0.42 & 237 \\
\hline $\mathrm{Ru}-\mathrm{KNH}_{2}$ & $0.8 / 20$ & GNP & & 15000 & 12000 & 10 & 375 & 96 & & 53 & & 235 \\
\hline $\mathrm{Ru}-\mathrm{LiNH}_{2}$ & 5 & $\mathrm{MgO}$ & & 60000 & & 5 & 400 & 100 & & 53 & & 232 \\
\hline $\mathrm{Ru}-\mathrm{Mg}\left(\mathrm{NH}_{2}\right)_{2}$ & 5.0 & & & 60000 & & 100 & 400 & 3 & 0.02 & 101 & 0.14 & 237 \\
\hline $\mathrm{Ru}-\mathrm{NaNH}_{2}$ & $0.8 / 20$ & GNP & & 15000 & 12000 & 10 & 375 & 97 & & 53 & & 235 \\
\hline
\end{tabular}

catalysts composed of $\mathrm{MnN}$ and amides of the metals $\mathrm{Li}, \mathrm{Na}$ and $\mathrm{K}$ through mechanochemical methods, obtaining significantly higher catalytic activity with $\mathrm{MnN}-\mathrm{LiNH}_{2}$. The three alkali metals used have shown an ability to promote the decomposition of ammonia $(\mathrm{Li}>\mathrm{K}>\mathrm{Na}$ ), which is reversed with respect to when they were used as promoters in other formulations. Therefore, in the case of being linked with $-\mathrm{NH}_{2}$, these metals participate in the reaction as cocatalyst, instead of having only an electronic influence. ${ }^{378}$ In this context, Guo et al. ${ }^{379}$ analyzed the catalytic activity of $\mathrm{MnN}$ combined with the imide $\mathrm{Li}_{2} \mathrm{NH}$ and compared the results with a $\mathrm{Ru} /$ CNTs catalyst, obtaining a higher activity with the $\mathrm{MnN}-\mathrm{Li}_{2} \mathrm{NH}$ catalyst under the same reaction conditions. The nitride $\mathrm{Mn}_{6} \mathrm{~N}_{5}$, which is almost inactive in the decomposition of ammonia, showed relatively high catalytic activity when mixed with the imide $\mathrm{CaNH}{ }^{380}$

Other types of catalysts have been prepared by adding amides to ruthenium, typically using mechanochemical methods, such as the alkaline earth metal amides of $\mathrm{Ba}, \mathrm{Ca}$, and $\mathrm{Mg} .{ }^{237}$ Catalytic tests indicated that the best catalytic activity is reached with $\mathrm{Ru}-$ $\mathrm{Ba}\left(\mathrm{NH}_{2}\right)_{2}$ and $\mathrm{Ru}-\mathrm{Ca}\left(\mathrm{NH}_{2}\right)_{2}$ with respect to $\mathrm{Ru}-\mathrm{Mg}\left(\mathrm{NH}_{2}\right)_{2}$ and to the reference catalyst $\mathrm{Ru} / \mathrm{MgO}$. Similarly, Guo et al. used $\mathrm{MgO}$ as a support for $\mathrm{Ru}-\mathrm{LiNH}_{2}$ and compared the catalytic results with $\mathrm{Ru} / \mathrm{CNT}$ and $\mathrm{Ru} / \mathrm{MgO}$; the latter was also promoted with $\mathrm{K}^{232}$ The highest catalytic activity was reached with $\mathrm{Ru}-\mathrm{LiNH}_{2} / \mathrm{MgO}$, followed by the $\mathrm{Ru}$ catalyst supported on carbon nanotubes, the catalyst supported on $\mathrm{MgO}$ promoted with $\mathrm{K}$, and finally, the nonpromoted $\mathrm{Ru} / \mathrm{MgO}$. Furthermore, with the catalyst composed of lithium imide and ruthenium, complete ammonia conversion was achieved at $400{ }^{\circ} \mathrm{C}$ with an ammonia concentration of 5 vol \%. In addition, Chang et al. ${ }^{235}$ tested $\mathrm{Ru}$ and $\mathrm{Ni}$ catalysts with $\mathrm{Na}$ and $\mathrm{K}$ amides supported on graphite nanoplatelets (GNP), obtaining very high ammonia conversion with respect to the same samples prepared through ball milling. Additionally, they were able to compare the effect of doping the $\mathrm{NaNH}_{2}$ and $\mathrm{KNH}_{2}$ amides with $\mathrm{Na}$ and $\mathrm{K}$ used as promoters of the $\mathrm{Ru} / \mathrm{GNP}$ catalyst, obtaining a conversion $53 \%$ higher in the case of $\mathrm{Ru}-\mathrm{NaNH}_{2}$ and $34 \%$ in the case of $\mathrm{Ru}$ - 
$\mathrm{KNH}_{2}$ at temperatures as low as $375{ }^{\circ} \mathrm{C}$ with respect to $\mathrm{Ru} /$ GNP promoted with $\mathrm{Na}$ and $\mathrm{K}$. The use of the $\mathrm{Na}$ and $\mathrm{K}$ promoters had already allowed an increase in the ammonia conversion of 9 and $27 \%$, respectively, with respect to the nonpromoted catalyst. It is worth mentioning that doping $\mathrm{Ni}$ / GNP with $\mathrm{Na}$ amide allowed a higher conversion with respect to $\mathrm{KNH}_{2}\left(44\right.$ vs $33 \%$ at $375{ }^{\circ} \mathrm{C}$ ), although the catalyst undergoes deactivation at lower temperatures.

An exhaustive study on the catalytic activity of catalysts composed of lithium imides and transition metal nitrides (TMN) was carried out by preparing a series of catalysts from the respective chlorides of the transition metals and $\mathrm{LiNH}_{2}$, and a successive nitriding process with $\mathrm{NH}_{3}{ }^{233}$ The $\mathrm{Li}_{2} \mathrm{NH}-\mathrm{Fe}_{2} \mathrm{~N}$ catalyst exhibits a higher ammonia decomposition rate with respect to $\mathrm{Fe}_{2} \mathrm{~N}, \mathrm{Li}_{2} \mathrm{NH}$, and $\mathrm{Fe}$ supported on CNTs. The relative order of the catalytic activity of the transition metals in $\mathrm{Li}_{2} \mathrm{NH}-\mathrm{TMN}$ at $500{ }^{\circ} \mathrm{C}$ is $\mathrm{Mn}>\mathrm{Co} \approx \mathrm{Fe} \approx \mathrm{Cr}>\mathrm{Ni}>\mathrm{V}>\mathrm{Ti} \approx$ $\mathrm{Cu}$. In their mixed forms with lithium imide, the nitrides of the transition metals showed a significantly higher activity compared to the form without $\mathrm{Li}_{2} \mathrm{NH}$. In the case of $\mathrm{Co}, \mathrm{Ni}$, and $\mathrm{Cu}$, the catalysts were compared with their supported form on CNTs. Using the catalyst $\mathrm{Li}_{2} \mathrm{NH}-\mathrm{MnN}$, a higher activity was reported with respect to $\mathrm{Ru} / \mathrm{CNTs}$. Wang et al. ${ }^{359}$ prepared a catalyst composed of $\mathrm{Li}_{3} \mathrm{FeN}_{2}$ with which they obtained a $27 \%$ higher conversion than with a $\mathrm{Fe}_{2} \mathrm{~N}$ catalyst. Cao et al. ${ }^{360}$ tested catalysts containing ternary amides of the alkali metals $\mathrm{K}, \mathrm{Rb}$, and $\mathrm{Mn}$ prepared with mechanochemical methods. The results showed that $\mathrm{Rb}_{2}\left[\mathrm{Mn}\left(\mathrm{NH}_{2}\right)_{4}\right]$ has a catalytic activity higher than $\mathrm{K}_{2}\left[\mathrm{Mn}\left(\mathrm{NH}_{2}\right)_{4}\right]$ and also higher than a $\mathrm{Ru} / \mathrm{MgO}$ reference catalyst. Catalysts with metal amides and imides as active phase used to decompose ammonia and their catalytic performances are reported in Table 10.

3.2. Kinetics and Reaction Mechanism. In recent years, a large number of studies have been carried out on the kinetics of ammonia decomposition using different catalysts to determine the expression of the reaction rate and to understand the decomposition mechanism. The first kinetic expressions proposed considered that the decomposition of ammonia followed a first order kinetics, ${ }^{381,382}$ which in some cases is still used today. ${ }^{131,184,250,317,321,341}$ However, other authors proposed that the reaction rate is a combination of zero order and first order as a function of temperature, ${ }^{383,384}$ while more recent studies have shown that the reaction is inhibited by the presence of $\mathrm{H}_{2}{ }^{238,385}$ The inhibition is manifested particularly at low temperatures, and it has been proposed that it is due to the hydrogen that accumulates on the surface of the catalyst. ${ }^{143}$ Different methods have been used to determine the expression of the reaction rate; on the one hand, the LangmuirHinshelwood-Hougen-Watson (LHHW) approach, which considers coverage-independent parameters. ${ }^{166,295,386-389}$ On the other hand, the Temkin-Pyzhev model considers that the associative desorption of nitrogen is the rate-limiting step, and that if the influence of the inverse reaction can be neglected the reaction rate is expressed as a power law. ${ }^{97,390-392}$ Finally, the Tamaru model considers that a different model is applied depending on the pressure and temperature conditions. ${ }^{370,393,394}$ The model proposed by Tamaru et al. was verified for a W catalyst ${ }^{395-397}$ and confirmed to be valid for other types of catalysts as well, such as $\mathrm{Pt},{ }^{398} \mathrm{VN},{ }^{384} \mathrm{Mo}_{2} \mathrm{~N},{ }^{345}$ and $\mathrm{TiN}_{x} \mathrm{O}_{y}{ }^{394}$ To determine the parameters of the rate expression, a parameter estimation problem can be applied. This is done by testing different kinetic expressions obtained by alternatively considering each elementary step of the reaction as the rate limiting step to find the one that best fits the experimental results, considering all the other steps in equilibrium (quasi-equilibrium approach). ${ }^{399}$

As proposed by Temkin, ${ }^{400}$ the mechanism considered for the decomposition of ammonia occurs following the opposite steps of ammonia synthesis and starts with the adsorption of ammonia on the catalyst surface, followed by subsequent dehydrogenation steps, and finally nitrogen and hydrogen atoms desorb as $\mathrm{N}_{2}$ and $\mathrm{H}_{2}$ (eq 1-6):

$$
\begin{aligned}
& \mathrm{NH}_{3(\mathrm{~g})}+\mathrm{s} \leftrightarrows \mathrm{NH}_{3(\mathrm{a})} \\
& \mathrm{NH}_{3(\mathrm{a})}+\mathrm{s} \leftrightarrows \mathrm{NH}_{2(\mathrm{a})}+\mathrm{H}_{(\mathrm{a})} \\
& \mathrm{NH}_{2(\mathrm{a})}+\mathrm{s} \leftrightarrows \mathrm{NH}_{(\mathrm{a})}+\mathrm{H}_{(\mathrm{a})} \\
& \mathrm{NH}_{(\mathrm{a})}+\mathrm{s} \leftrightarrows \mathrm{N}_{(\mathrm{a})}+\mathrm{H}_{(\mathrm{a})} \\
& 2 \mathrm{~N}_{(\mathrm{a})} \leftrightarrows \mathrm{N}_{2(\mathrm{~g})}+2 \mathrm{~s} \\
& 2 \mathrm{H}_{(\mathrm{a})} \leftrightarrows \mathrm{H}_{2(\mathrm{~g})}+2 \mathrm{~s}
\end{aligned}
$$

where s represents an empty site on the catalyst surface and the subscript (a) refers to a species adsorbed on the surface.

The first analyses of the reaction kinetics were based on the concept that in the synthesis of ammonia the limiting step of the mechanism is the cleavage of the $\mathrm{N}-\mathrm{N}$ bond of the nitrogen molecule. Therefore, recombinative desorption of $\mathrm{N}_{2}$ was proposed as the limiting step of the ammonia decomposition reaction. Logan and Kemball in 1959 analyzed the reaction rates of several catalysts and their dependence on the pressure of ammonia and hydrogen, concluding that, for all the catalysts tested (Ni, Co, Rh, Pt, Ru, Re, Fe, VN, and W) the step that determines the reaction rate is the desorption of nitrogen from the catalyst surface. ${ }^{401}$ Actually, the limiting step of the reaction can vary depending on different properties of the catalyst, such as its composition or the architecture of the active sites. For noble metals such as $\mathrm{Ru}, \mathrm{Rh}, \mathrm{Ir}, \mathrm{Pt}, \mathrm{Pd}$, or $\mathrm{Cu}$, the cleavage of $\mathrm{N}-$ $\mathrm{H}$ bonds was proposed as a limiting step, ${ }^{166}$ while for metals such as $\mathrm{Fe}, \mathrm{Co}, \mathrm{Ni}$, or $\mathrm{Cr}$ it seems to be the desorption of nitrogen. ${ }^{155}$ However, Takahashi and Fujitani ${ }^{238}$ obtained a different result analyzing the kinetics of two catalysts composed of $\mathrm{Ni}$ or $\mathrm{Ru}$ supported on $\mathrm{MgO}$. They concluded that for $\mathrm{Ru}$, nitrogen desorption is the limiting step, as it requires a higher energy, while for $\mathrm{Ni}$ the limiting step is the total dehydrogenation of ammonia. This suggests that the catalyst support also has a strong influence on the reaction mechanism. In the case of $\mathrm{Ni}$, it was proposed that the dehydrogenation of $\mathrm{NH}_{3}$ is the determining step of the reaction rate in the case of supported catalysts with low $\mathrm{Ni}-\mathrm{N}$ binding energy, and the desorption of nitrogen in the case of high $\mathrm{Ni}-\mathrm{N}$ binding energy. ${ }^{255} \mathrm{~A}$ similar result was obtained by Hansgen et al.; ${ }^{402}$ they concluded that the determining step of the reaction rate is the elimination of the second hydrogen atom (eq 3) for surfaces with a $\mathrm{M}-\mathrm{N}$ bond energy lower than $125 \mathrm{kcal} \mathrm{mol}^{-1}$, while, for surfaces with higher $\mathrm{M}-\mathrm{N}$ bond energies, the removal of the first and second hydrogen atoms (eqs 2-3) and the desorption of nitrogen (eq 5) are kinetically significant. These authors developed a method to predict the composition of a bimetallic catalyst with high catalytic activity through the analysis of nitrogen binding energy values. They tested a large number of $\mathrm{Pt}$ compositions bound with $\mathrm{Ti}, \mathrm{V}, \mathrm{Cr}, \mathrm{Mn}, \mathrm{Fe}, \mathrm{Co}$, and $\mathrm{Ni}$, obtaining the energy closest to the optimum (the energy of $\mathrm{Ru}$ ) with catalysts $\mathrm{Pt}-\mathrm{Ni}-$ $\mathrm{Pt}(111), \mathrm{Fe}-\mathrm{Pt}-\mathrm{Pt}(111)$, and $\mathrm{Co}-\mathrm{Pt}-\mathrm{Pt}(111){ }^{403}$ Duan et al. $^{404}$ determined a linear relationship between the apparent 
activation energy of the nitrogen recombination reaction and the adsorption energy of $\mathrm{N}$ on the surfaces of $\mathrm{Fe}, \mathrm{Co}, \mathrm{Ni}$, and $\mathrm{Cu}$, known as the Brønsted-Evans-Polanyi relationship. The calculated apparent activation energy followed the order: $\mathrm{Fe}>$ $\mathrm{Co} \approx \mathrm{Ni}>\mathrm{Cu}$. However, by applying this method to $\mathrm{Ni}-\mathrm{Ru}$, $\mathrm{Ni}-\mathrm{Pt}$, and Ni-WC bimetallic catalysts, which have a similar nitrogen binding energy, Hansgen et al. ${ }^{405}$ concluded that this cannot be considered as the only determining factor to evaluate the catalytic activity, since the three compositions have different activity. In this sense, by comparing the binding energy of monometallic Ni and $\mathrm{Pt}$ catalysts with their bimetallic forms, Zhong et al. ${ }^{406}$ obtained a reduction in the activation energy for the bimetallic $\mathrm{Pt}-\mathrm{Ni}-\mathrm{Pt}(111)$ catalyst. Also, Zheng et al. ${ }^{236}$ considered the binding energy of $\mathrm{N}$ comparing the nitride and carbide forms of molybdenum, and concluded that although the binding energy of $\mathrm{N}$ is stronger on $\mathrm{MoN}$ than on $\mathrm{Mo}_{2} \mathrm{C}$, the former is more active due to a greater number of active sites.

One of the first experimental studies of the kinetics of the ammonia decomposition reaction was carried out in 1941 and it focused on a $\mathrm{Fe}$ catalyst promoted with $\mathrm{Al}_{2} \mathrm{O}_{3}$ and $\mathrm{K}_{2} \mathrm{O}$, comparing its behavior with a catalyst promoted only with $\mathrm{Al}_{2} \mathrm{O}_{3}$, and with an nonpromoted $\mathrm{Fe}$ catalyst. ${ }^{407}$ The reaction rate of $\mathrm{Fe}\left(\mathrm{K}_{2} \mathrm{O}\right) / \mathrm{Al}_{2} \mathrm{O}_{3}$ showed a linear behavior with respect to temperature. It was proposed that the reaction rate depended on the pressure of ammonia and hydrogen, and that the limiting step in the entire temperature range was the desorption of nitrogen (eq 5). On the contrary, for the other two catalysts, the reaction rate showed a dependence on temperature, and depending on the reaction conditions the limiting step also varied, from the desorption of nitrogen to one of the last two dehydrogenations (eqs 3 and 4). Using a Fe catalyst promoted with $\mathrm{Al}_{2} \mathrm{O}_{3}, \mathrm{~K}_{2} \mathrm{O}$, and $\mathrm{SiO}_{2}$, Takezawa and Toyoshima ${ }^{408,409}$ determined a change in the limiting step of the reaction with increasing reaction temperature, from nitrogen desorption to dehydrogenation of $\mathrm{NH}_{2}$ on the catalyst surface.

By carrying out experiments with other catalysts at low pressures down to ultrahigh vacuum, the same behavior was observed; that is, the limiting step of the reaction can vary, not only according to the type of catalyst and active phase, but also changing the reaction temperature. ${ }^{70,71}$ Furthermore, the activation energy can vary in the different ranges of reaction temperature, as in the case of the metals $\mathrm{Pt}, \mathrm{Ni}, \mathrm{Rh}, \mathrm{Ta}$, and $\mathrm{W}^{70,71}$ For example, with a Fe catalyst, a variation of the apparent activation energy was determined from a value of 188 $\mathrm{kJ} \mathrm{mol}{ }^{-1}$ at temperatures up to approximately $370{ }^{\circ} \mathrm{C}$, to $21 \mathrm{~kJ}$ $\mathrm{mol}^{-1}$ above $440{ }^{\circ} \mathrm{C}$, while at around $580{ }^{\circ} \mathrm{C}$ the reaction rate becomes practically independent from temperature. ${ }^{410}$ Kunsman in 1928 was already able to determine the change in apparent activation energy according to the reaction temperature for $\mathrm{Ni}, \mathrm{Mo}, \mathrm{W}$, and Fe catalysts. ${ }^{411,412}$ Using an expression similar to Temkin-Pyzhev, Lamb et al. ${ }^{225}$ determined for a Ru catalyst supported on alumina and promoted with $\mathrm{LiOH}$ an apparent activation energy of $83 \mathrm{~kJ} \mathrm{~mol}^{-1}$ up to $475^{\circ} \mathrm{C}$ and of 77 $\mathrm{kJ} \mathrm{mol}{ }^{-1}$ for higher temperatures. Also, the exponents of $\mathrm{NH}_{3}$ and $\mathrm{H}_{2}$ concentrations vary in the two temperature ranges, which are most likely due to a combination of factors, among which there is the inhibition effect of $\mathrm{H}_{2}$, which can also vary according to the temperature. Tsai and Weinberg ${ }^{382}$ reported a change in the limiting step of the reaction and the apparent activation energy for a $\mathrm{Ru}(001)$ surface, and McCabe ${ }^{383}$ reported a change in the limiting step of the reaction from the desorption of nitrogen to the adsorption of $\mathrm{NH}_{3}$ on the surface at high temperatures (greater than $727^{\circ} \mathrm{C}$ ) with a Ni catalyst.
Likewise, Rasim et al. $^{413}$ observed that, with the use of a Pt catalyst and a Pt-Au bimetallic catalyst at low temperature and high ammonia pressure, the decomposition rate of ammonia has a zero-order dependence on the ammonia pressure, while at high temperature the decomposition rate becomes almost independent of temperature. It is important to mention that the bimetallic catalyst showed superior potential to decompose ammonia. Similarly, Richardson et al. ${ }^{240}$ studied a Pt catalyst supported on alumina and confirmed that the mechanism previously proposed by Vajo et al., ${ }^{414}$ who stated that for high temperatures and/or low pressures, the determining step is the dissociation of the first $\mathrm{N}-\mathrm{H}$ bond, while, at low temperatures and high pressures, the nitrogen desorption is the limiting step and the reaction rate does not depend on the ammonia concentration. In contrast, Egawa et al. ${ }^{415}$ observed the opposite behavior with $\mathrm{Ru}$; at low temperatures the reaction rate follows the Temkin-Pyzhev mechanism and the cleavage of the $\mathrm{N}-\mathrm{H}$ bond is the determining step of the reaction, while at high temperature the reaction rate is proportional to the concentration of ammonia and independent of the concentrations of the products, the limiting step being the desorption of nitrogen. However, Choudhary et al., ${ }^{72}$ focusing on an $\operatorname{Ir}(100)$ surface in a pressure range from ultrahigh vacuum to 1.5 Torr, found a constant activation energy and that the limiting step of the reaction is the desorption of nitrogen for all the temperatures used. But a different result was obtained by Huang et al. ${ }^{416}$ using the same catalyst; the cleavage of the first two $\mathrm{N}-\mathrm{H}$ bonds (eqs 2 and 3) needs a similar energy, and those bonds are the determining steps of the reaction, while the desorption of nitrogen has a lower energy barrier than the dehydrogenation steps.

Analyzing the kinetics of the reaction on $\mathrm{Ru} / \mathrm{CNT}$ catalysts with different sizes of Ru particles, Zhou et al. ${ }^{417}$ observed that although in all cases the recombination of nitrogen is the determining step of the reaction, the apparent activation energy changed with the particle size. In a similar manner, but using a $\mathrm{Ru}$ catalyst supported on $\mathrm{Al}_{2} \mathrm{O}_{3}$, Zheng et al. ${ }^{217}$ found a linear relationship between the apparent activation energy and the mean size of $\mathrm{Ru}$ particles, with a higher apparent activation energy with small particle sizes.

Considering that the dehydrogenation of $\mathrm{NH}_{3}$ and the desorption of $\mathrm{N}_{2}$ are both the determining steps of the ammonia decomposition mechanism, Henpraserttae et al. ${ }^{277}$ proposed that by increasing the number of active sites of a Ni/ $\mathrm{Al}_{2} \mathrm{O}_{3}$ catalyst and its basicity, the dehydrogenation of $\mathrm{NH}_{3}$ would be promoted. At the same time, a high dispersion of Ni would allow a higher number of active sites to be close, increasing the possibility that the adsorbed nitrogen will recombine and desorb to form $\mathrm{N}_{2}$ gas. Chellappa et al. ${ }^{370}$ determined for a $\mathrm{Pt}-\mathrm{Ni}$ catalyst supported on alumina that at high temperatures $(>520$ ${ }^{\circ} \mathrm{C}$ ) the reaction rate could be expressed using a first order equation, and that under these conditions there is no inhibition by hydrogen. On a Ni/ $\mathrm{Al}_{2} \mathrm{O}_{3}$ catalyst, the inhibition effect of hydrogen on the reaction rate changes according to the temperature range. ${ }^{418}$ Similarly, using a Pt catalyst, Tsai et al. ${ }^{419}$ reported that hydrogen inhibition depends on temperature, and also on pressure. Comparing the reaction kinetics of $\mathrm{Fe}$ and Pt catalysts, Löffler and Schmidt concluded that hydrogen inhibition was strong for $\mathrm{Pt}^{420}$ while for $\mathrm{Fe}$ the inhibition was observed only at low temperatures and high pressures. ${ }^{421}$ For a Ru-ceria catalyst supported on $\mathrm{Y}_{2} \mathrm{O}_{3}-\mathrm{ZrO}_{2}$ the decomposition rate is first order in ammonia, and hydrogen 
Table 11. Catalysts Used to Study the Kinetics of Ammonia Decomposition, The Limiting Step of the Reaction Mechanism, and Apparent Activation Energy. The Limiting Step of the Reaction Refers to the Steps Described in eq 1 to 6

\begin{tabular}{|c|c|c|c|c|c|}
\hline active phase & support & promoter & $\begin{array}{l}\text { rate limiting } \\
\text { step }\end{array}$ & $\begin{array}{l}\text { activation energy } \\
\left(\mathrm{kJ} \mathrm{mol}{ }^{-1}\right)\end{array}$ & ref \\
\hline $\mathrm{Pt}$ & & & $2,2-4,3,4,5$ & $69-247$ & $70,166,398,401-403,406,413,414,419,421,426,428-430,435$ \\
\hline $\mathrm{Ta}$ & & & & 59 & 71 \\
\hline $\mathrm{Rh}$ & & & $2,2-4,4,5$ & $88-238$ & $71,166,401,426,436$ \\
\hline $\mathrm{Ni}$ & & & 2,5 & $70-286$ & $71,255,270,383,401,402,404,406,412,427-429,432,437-444$ \\
\hline $\mathrm{W}$ & & & 4,5 & $87-300$ & $71,396,401,412,445,446$ \\
\hline Ir & & & $2,3,2-4,5$ & $64-131$ & $72,166,416,447,448$ \\
\hline $\mathrm{Ru}$ & $\mathrm{CaO}$ & & & 96 & 73 \\
\hline $\mathrm{Ru}$ & $\mathrm{CaO}$ & $\mathrm{K}$ & & 75 & 73 \\
\hline $\mathrm{Ru}$ & $\mathrm{Al}_{2} \mathrm{O}_{3}$ & & $2,3,2-4,4,5$ & $92-130$ & $74,155,217,220,221,393,424,449$ \\
\hline Co & $\mathrm{MgAl}_{2} \mathrm{O}_{4}$ & & 5 & & 153 \\
\hline $\mathrm{Cu}$ & $\mathrm{MgAl}_{2} \mathrm{O}_{4}$ & & 5 & & 153 \\
\hline $\mathrm{Fe}$ & $\mathrm{MgAl}_{2} \mathrm{O}_{4}$ & & 5 & & 153 \\
\hline $\mathrm{Ni}$ & $\mathrm{MgAl}_{2} \mathrm{O}_{4}$ & & 5 & & 153 \\
\hline $\mathrm{Ru}$ & $\mathrm{MgAl}_{2} \mathrm{O}_{4}$ & & 5 & & 153 \\
\hline $\mathrm{Co}_{3} \mathrm{Mo}_{3} \mathrm{~N}$ & & & 5 & 70 & 153,350 \\
\hline $\mathrm{Cr}$ & $\mathrm{Al}_{2} \mathrm{O}_{3}$ & & 5 & & 155 \\
\hline $\mathrm{Cu}$ & $\mathrm{Al}_{2} \mathrm{O}_{3}$ & & 4 & & 155 \\
\hline $\operatorname{Ir}$ & $\mathrm{Al}_{2} \mathrm{O}_{3}$ & & 4 & & 155 \\
\hline $\mathrm{Pb}$ & $\mathrm{Al}_{2} \mathrm{O}_{3}$ & & & & 155 \\
\hline $\mathrm{Se}$ & $\mathrm{Al}_{2} \mathrm{O}_{3}$ & & & & 155 \\
\hline $\mathrm{Te}$ & $\mathrm{Al}_{2} \mathrm{O}_{3}$ & & & & 155 \\
\hline $\mathrm{Pt}$ & $\mathrm{Al}_{2} \mathrm{O}_{3}$ & & $2,4,5$ & & 155,240 \\
\hline $\mathrm{Ni}$ & $\mathrm{Al}_{2} \mathrm{O}_{3}$ & & $2-4,5$ & $88-96$ & $155,251,254,255,257,276,393,418$ \\
\hline Co & $\mathrm{Al}_{2} \mathrm{O}_{3}$ & & $2-4,5$ & & 155,393 \\
\hline $\mathrm{Fe}$ & $\mathrm{Al}_{2} \mathrm{O}_{3}$ & & 5 & 212 & 155,407 \\
\hline $\mathrm{Pd}$ & $\mathrm{Al}_{2} \mathrm{O}_{3}$ & & 4 & 132 & 155,449 \\
\hline $\mathrm{Rh}$ & $\mathrm{Al}_{2} \mathrm{O}_{3}$ & & 4 & 126 & 155,449 \\
\hline $\mathrm{Pd}$ & & & $2,2-4,4$ & $37-130$ & $166,426,427,431,447$ \\
\hline $\mathrm{Ni}$ & $\mathrm{La}-\mathrm{Al}_{2} \mathrm{O}_{3}$ & & 5 & 98 & 251 \\
\hline $\mathrm{Ni}$ & $\mathrm{Gd}_{2} \mathrm{O}_{3}$ & & & & 254 \\
\hline $\mathrm{Ni}$ & $\mathrm{Sm}_{2} \mathrm{O}_{3}$ & & & & 254 \\
\hline $\mathrm{Ni}$ & $\mathrm{CeO}_{2}$ & & & & 254,255 \\
\hline $\mathrm{Ni}$ & $\mathrm{La}_{2} \mathrm{O}_{3}$ & & & & 254,255 \\
\hline $\mathrm{Ni}$ & $\mathrm{Y}_{2} \mathrm{O}_{3}$ & & & & 254,255 \\
\hline $\mathrm{Ni}$ & $\mathrm{ZrO}_{2}$ & & & & 255 \\
\hline $\mathrm{Ni}$ & $\mathrm{MgO}$ & & $2-4,3$ & 144 & 238,255 \\
\hline $\mathrm{Ni}$ & $\mathrm{Al}_{2} \mathrm{O}_{3}$ & $\mathrm{Ce}$ & & & 257 \\
\hline $\mathrm{Ni}$ & $\mathrm{Al}_{2} \mathrm{O}_{3}$ & $\mathrm{Eu}$ & & & 257 \\
\hline $\mathrm{Ni}$ & $\mathrm{Al}_{2} \mathrm{O}_{3}$ & Gd & & & 257 \\
\hline $\mathrm{Ni}$ & $\mathrm{Al}_{2} \mathrm{O}_{3}$ & $\mathrm{La}$ & & & 257 \\
\hline $\mathrm{Ni}$ & $\mathrm{Al}_{2} \mathrm{O}_{3}$ & $\mathrm{Nd}$ & & & 257 \\
\hline $\mathrm{Ni}$ & $\mathrm{Al}_{2} \mathrm{O}_{3}$ & $\operatorname{Pr}$ & & & 257 \\
\hline $\mathrm{Ni}$ & $\mathrm{Al}_{2} \mathrm{O}_{3}$ & $\mathrm{Sm}$ & & & 257 \\
\hline $\mathrm{Ni}$ & $\mathrm{Al}_{2} \mathrm{O}_{3}$ & $\mathrm{Y}$ & & & 257 \\
\hline $\mathrm{Ni}$ & $\mathrm{ZrO}_{2}-\mathrm{Al}_{2} \mathrm{O}_{3}$ & & 2,5 & 86 & 276 \\
\hline $\mathrm{Mo}_{2} \mathrm{C}$ & & & 5 & 89 & 236 \\
\hline $\mathrm{Mo}_{2} \mathrm{~N}$ & & & 5 & $97-251$ & $236,349,350,450$ \\
\hline $\mathrm{ZnON}$ & & & 5 & 187 & 319 \\
\hline $\mathrm{Fe}$ & & $\mathrm{Al}, \mathrm{Ca}$ & 5 & 167 & 295 \\
\hline $\mathrm{Fe}$ & & $\mathrm{Al}, \mathrm{Ca}, \mathrm{K}$ & 5 & $93-146$ & $295,302-304,320$ \\
\hline $\mathrm{Fe}_{4} \mathrm{~N}$ & & & & 143 & 299 \\
\hline $\mathrm{Fe}$ & & & $1,2,3$ or 4,5 & $68-275$ & $299,324,400,401,403,404,407,410,421,430,437,451$ \\
\hline $\mathrm{Fe}$ & & $\mathrm{Al}, \mathrm{Ca}, \mathrm{K}, \mathrm{Si}$ & 5 & & 301 \\
\hline Co & & & 4,5 & $111-180$ & $324,400,401,403,404$ \\
\hline $\mathrm{Fe}_{3} \mathrm{Mo}_{3} \mathrm{~N}$ & & & 5 & $73-89$ & 349,350 \\
\hline $\mathrm{Ni}_{3} \mathrm{Mo}_{3} \mathrm{~N}$ & & & & 84 & 350 \\
\hline Co-Mo & $\mathrm{Al}_{2} \mathrm{O}_{3}$ & & 5 & 106 & 366 \\
\hline $\mathrm{Ni}-\mathrm{Pt}$ & $\mathrm{Al}_{2} \mathrm{O}_{3}$ & & 5 & 47 & 370 \\
\hline $\mathrm{Li}_{2} \mathrm{NH}-\mathrm{Fe}_{2-4} \mathrm{~N}$ & & & & & 376 \\
\hline
\end{tabular}


Table 11. continued

\begin{tabular}{|c|c|c|c|c|c|}
\hline active phase & support & promoter & $\begin{array}{l}\text { rate limiting } \\
\text { step }\end{array}$ & $\begin{array}{l}\text { activation energy } \\
\left(\mathrm{kJ} \mathrm{mol}{ }^{-1}\right)\end{array}$ & ref \\
\hline $\mathrm{Li}_{2} \mathrm{NH}-\mathrm{MnN}$ & & & 6 & 75 & 376,379 \\
\hline Mn & & & & & 377 \\
\hline $\mathrm{Ru}$ & & & 5 & $91-209$ & $382,401,415,425,430,452,453$ \\
\hline $\mathrm{Fe}$ & & $\mathrm{Ca}, \mathrm{K}$ & & & 321 \\
\hline $\mathrm{Fe}$ & & $\mathrm{K}$ & & & 321 \\
\hline VN & & & 5 & $135-209$ & $384,401,454$ \\
\hline $\mathrm{Ru}$ & $\mathrm{MgO}$ & & 5 & 124 & 238 \\
\hline $\mathrm{Fe}-\mathrm{Cr}$ & $\mathrm{Al}_{2} \mathrm{O}_{3}$ & & $2-4$ & & 393 \\
\hline $\mathrm{TiN}_{x} \mathrm{O}_{y}$ & & & 5 & 215 & 394 \\
\hline $\mathrm{Co}-\mathrm{Fe}$ & & & 5 & & 400 \\
\hline $\operatorname{Re}$ & & & 5 & $137-259$ & $401,430,455$ \\
\hline $\mathrm{Cr}-\mathrm{Pt}$ & & & & & 402 \\
\hline $\mathrm{Mn}-\mathrm{Pt}$ & & & & & 402 \\
\hline $\mathrm{Pt}-\mathrm{Mn}$ & & & & & 402 \\
\hline $\mathrm{Pt}-\mathrm{Ti}$ & & & & & 402 \\
\hline $\mathrm{Pt}-\mathrm{V}$ & & & & & 402 \\
\hline $\mathrm{Ti}-\mathrm{Pt}$ & & & & & 402 \\
\hline $\mathrm{V}-\mathrm{Pt}$ & & & & & 402 \\
\hline $\mathrm{Co}-\mathrm{Pt}$ & & & & & $402,403,429$ \\
\hline $\mathrm{Fe}-\mathrm{Pt}$ & & & & & $402,403,429$ \\
\hline $\mathrm{Ni}-\mathrm{Pt}$ & & & 2,5 & 42 & $402,405,406,428,429$ \\
\hline $\mathrm{Cu}-\mathrm{Pt}$ & & & & & 403 \\
\hline $\mathrm{Cu}$ & & & 2,4 & $87-317$ & $403,431,456,457$ \\
\hline $\mathrm{Ni}-\mathrm{Ru}$ & & & & & 405 \\
\hline Ni-WC & & & & & 405 \\
\hline $\mathrm{Fe}$ & & $\mathrm{Al}$ & 3 or 4,5 & & 407 \\
\hline $\mathrm{Fe}$ & & $\mathrm{Al}, \mathrm{K}$ & 5 & $183-191$ & $407,411,458$ \\
\hline $\mathrm{Fe}$ & & $\mathrm{Al}, \mathrm{K}, \mathrm{Si}$ & 3,5 & & 409 \\
\hline $\mathrm{Fe}$ & & $\mathrm{Al}, \mathrm{Sn}$ & & & 411 \\
\hline Mo & & & & & 412 \\
\hline $\mathrm{Ru}$ & $\mathrm{Al}_{2} \mathrm{O}_{3}$ & $\mathrm{LiOH}$ & & $77-83$ & 225 \\
\hline $\mathrm{Pt}-\mathrm{Au}$ & & & 2 & 93 & 413 \\
\hline $\mathrm{Ru}$ & CNTs & & 5 & 82 & 417 \\
\hline $\mathrm{Ru}$ & $\mathrm{CeO}_{2}-\mathrm{YZ}$ & & 5 & 67 & 422 \\
\hline $\mathrm{Ru}$ & $\mathrm{C}$ & & & 88 & 424 \\
\hline $\mathrm{Cu}-\mathrm{Pd}$ & & & 4 & $96-275$ & 431 \\
\hline $\mathrm{Ni}$ & $\mathrm{SiO}_{2}$ & & & 81 & 432 \\
\hline $\mathrm{Ni}-\mathrm{Pd}$ & & & & 59 & 432 \\
\hline $\mathrm{Pd}$ & $\mathrm{SiO}_{2}$ & & & 92 & 432 \\
\hline $\mathrm{Mo}_{3} \mathrm{~N}_{2}$ & & & 3 & 172 & 433 \\
\hline $\mathrm{NaNH}_{2}$ & & & 4,6 & & 434 \\
\hline $\mathrm{GaN}$ & & & & & 459 \\
\hline $\mathrm{Ir}$ & $\mathrm{AC}$ & & & & 460 \\
\hline $\mathrm{Ru}$ & $\mathrm{AC}$ & & & & 460 \\
\hline
\end{tabular}

has a negligible inhibitory effect on the decomposition rate below $320^{\circ} \mathrm{C} .{ }^{422}$

Wang et al. ${ }^{400}$ examined the behaviors of $\mathrm{Fe}, \mathrm{Co}$, and the $\mathrm{Fe}-$ Co bimetallic catalysts, determining that these three metals differ intrinsically. For $\mathrm{Fe}$, the adsorption of ammonia was shown to be the limiting step of the reaction, while for Co both the cleavage of the $\mathrm{N}-\mathrm{H}$ bond and the desorption of $\mathrm{N}_{2}$ are slow. For $\mathrm{Co}-\mathrm{Fe}$, nitrogen desorption from the catalyst surface was determined as the limiting step. Prasad et al. ${ }^{220}$ analyzed the kinetics of the reaction on $\mathrm{Ru} / \mathrm{Al}_{2} \mathrm{O}_{3}$ using different models. They concluded that there is no single limiting step, but that both $\mathrm{N}_{2}$ desorption and $\mathrm{NH}_{x}$ dehydrogenation reactions are kinetically significant. ${ }^{221,423}$ Moreover, for a $\mathrm{Ru} / \mathrm{C}$ catalyst a dependence on $\mathrm{NH}_{3}$ concentration close to the first order and a strong $\mathrm{H}_{2}$ inhibition were observed. ${ }^{424}$ The model that best fit the reaction kinetics involved both the cleavage of the $\mathrm{NH}_{2}-\mathrm{H}$ bond and the desorption of nitrogen. Observing the kinetics of the reaction on $\mathrm{Ru}$, Mortensen et al. ${ }^{425}$ proposed that the mechanism had to consider the diffusion of the adsorbed $\mathrm{NH}_{3}$ and the diffusion of the reaction products from the active sites, for which the latter step determines the reaction rate.

The analysis of the kinetics of the reaction and the estimation of the apparent activation energy would also make it possible to foresee the formulation of the catalyst that would lead to a higher performance in the conversion of ammonia. For example, by analyzing the kinetics of the decomposition reaction on different exposed faces of $\mathrm{Rh}, \mathrm{Pt}$, and $\mathrm{Pd}$, Novell-Leruth et al. ${ }^{426}$ determined that the first dehydrogenation (eq 2) is the determining step for $\mathrm{Pt}(100)$ and $\mathrm{Pd}(100)$, while on $\mathrm{Rh}(100)$ the dehydrogenation of $\mathrm{NH}_{3}$ is relatively fast and the 
Table 12. Structured Catalysts Used to Decompose Ammonia and Their Catalytic Performances

\begin{tabular}{|c|c|c|c|c|c|c|c|c|c|c|}
\hline structured catalyst & catalyst & $\begin{array}{c}P \\
(\text { bar })\end{array}$ & $\begin{array}{l}\text { reactor vol } \\
\quad(\mathrm{mL})\end{array}$ & $\begin{array}{l}\text { total flow } \\
\left(\mathrm{mL} \min ^{-1}\right)\end{array}$ & $\begin{array}{l}\% \mathrm{NH}_{3} \\
\text { inlet flow }\end{array}$ & $\begin{array}{c}T \\
\left({ }^{\circ} \mathrm{C}\right)\end{array}$ & $\begin{array}{c}\text { conv } \\
(\%)\end{array}$ & $\underset{\mathrm{mol}_{\text {met }}}{\mathrm{mol} \mathrm{H}_{2}} \mathrm{~s}^{-1}$ & $\begin{array}{c}E_{\mathrm{a}} \\
\left.(\mathrm{kJ} \mathrm{mol})^{-1}\right)\end{array}$ & ref \\
\hline $\mathrm{SiN}_{\mathrm{X}}$ reactor & $\mathrm{Ir} / \mathrm{Al}_{2} \mathrm{O}_{3}$ & 1 & 0.12 & 6 & 100 & 600 & 12 & & & 103 \\
\hline $\begin{array}{l}\text { cordierite monolith covered } \\
\text { with } \mathrm{Al}_{2} \mathrm{O}_{3}\end{array}$ & 5 wt $\% \mathrm{Ru}$ & 1 & & 100 & 100 & 500 & 45 & & 104 & 385 \\
\hline SiCN monolith & $\mathrm{Ru}$ & 1 & & 2 & 100 & 600 & 80 & & & 462 \\
\hline $\mathrm{CeO}_{2}$ monolith & 0.7 wt $\% \mathrm{Ni}$ & 1 & 1 & 25 & 57 & 500 & 82 & & & 463 \\
\hline cordierite monolith & 5 wt $\% \mathrm{Ni} / \mathrm{CeO}_{2}$ & 1 & 1 & 25 & 57 & 500 & 53 & & & 463 \\
\hline $\begin{array}{l}\text { microcanales covered with } \\
\mathrm{Al}_{2} \mathrm{O}_{3}\end{array}$ & $\begin{array}{l}3.5 \text { wt \% Ru- } 0.8 \text { wt } \\
\% \mathrm{~K}\end{array}$ & 1 & 0.35 & 145 & 100 & 525 & 88 & & & 464 \\
\hline $\begin{array}{l}\text { microchannels covered with } \\
\mathrm{Al}_{2} \mathrm{O}_{3}\end{array}$ & 3.5 wt $\% \mathrm{Ru}$ & 1 & 0.35 & 145 & 100 & 525 & 75 & & & 464 \\
\hline $\begin{array}{l}\text { microreactor with posts covered } \\
\text { with } \mathrm{Al}_{2} \mathrm{O}_{3}\end{array}$ & 3.5 wt $\% \mathrm{Ru}$ & 1 & 0.25 & 145 & 100 & 525 & 51 & & & 464 \\
\hline $\begin{array}{l}\mathrm{SiC} \text { monolith covered with } \\
\mathrm{Al}_{2} \mathrm{O}_{3}\end{array}$ & 5.8 wt $\% \mathrm{Ru}$ & 1 & 0.55 & 5 & 100 & 500 & 99 & & & 465 \\
\hline stainless steel microchannels & $\begin{array}{l}4.7 \text { wt } \% \mathrm{Ni}-0.1 \text { wt } \% \\
\mathrm{Pt} / \mathrm{Al}_{2} \mathrm{O}_{3}\end{array}$ & 0.9 & 0.36 & 50 & 100 & 700 & 99 & & 205 & 466,381 \\
\hline stainless steel microchannels & $\begin{array}{l}8.5 \text { wt \% Ru-Cs/ } \\
\mathrm{Al}_{2} \mathrm{O}_{3}\end{array}$ & 0.9 & 0.36 & 100 & 100 & 500 & 99 & & 117 & 467,468 \\
\hline silica plate covered with $\mathrm{Al}_{2} \mathrm{O}_{3}$ & 0.4 wt \% Co & 1 & 0.002 & & 50 & 575 & & 11.4 & & 469 \\
\hline silica plate covered with $\mathrm{Al}_{2} \mathrm{O}_{3}$ & 0.9 wt $\% \mathrm{Pd}$ & 1 & 0.002 & & 50 & 575 & & 6.4 & & 469 \\
\hline silica plate covered with $\mathrm{Al}_{2} \mathrm{O}_{3}$ & $1.0 \mathrm{wt} \% \mathrm{Fe}$ & 1 & 0.002 & & 50 & 575 & & 2.1 & & 469 \\
\hline silica plate covered with $\mathrm{Al}_{2} \mathrm{O}_{3}$ & 1.3 wt $\% \mathrm{Ru}$ & 1 & 0.002 & & 50 & 575 & & 2.5 & & 469 \\
\hline silica plate covered with $\mathrm{Al}_{2} \mathrm{O}_{3}$ & 7.9 wt $\% \mathrm{Ru}-\mathrm{Ba}$ & 1 & 0.002 & & & & & & & 469 \\
\hline silica plate covered with graphite & 10 wt $\% \mathrm{Ru}$ & 1 & 0.004 & & & & & & & 470 \\
\hline silica plate covered with graphite & $\begin{array}{l}7.2 \text { wt \% Ru-28.3 wt } \\
\% \text { Cs }\end{array}$ & 1 & 0.004 & & & & & & & 470 \\
\hline silica plate covered with graphite & $\begin{array}{l}8.8 \text { wt } \% \text { Ru-12 wt \% } \\
\text { Ba }\end{array}$ & 1 & 0.004 & & & & & & & 470 \\
\hline $\begin{array}{l}\text { Cordierite monolith covered } \\
\text { with } \mathrm{Al}_{2} \mathrm{O}_{3}\end{array}$ & 15 wt $\% \mathrm{Ni}$ & 1 & & 100 & 100 & 500 & 20 & & 153 & 471 \\
\hline $\begin{array}{l}\text { cordierite monolith covered } \\
\text { with } \mathrm{Al}_{2} \mathrm{O}_{3}\end{array}$ & 0.18 wt $\% \mathrm{Ru}$ & & & & & & & & & 472 \\
\hline $\begin{array}{l}\text { cordierite monolith covered } \\
\text { with } \mathrm{Al}_{2} \mathrm{O}_{3}\end{array}$ & 0.26 wt $\% \mathrm{Ru} / \mathrm{CNFs}$ & & & & & & & & & 472 \\
\hline $\begin{array}{l}\text { cordierite monolith covered } \\
\text { with } \mathrm{Al}_{2} \mathrm{O}_{3}\end{array}$ & $\begin{array}{l}0.30 \text { wt \% Ru/N- } \\
\text { CNFs }\end{array}$ & & & & & & & & & 472 \\
\hline $\begin{array}{l}\mathrm{TiO}_{2} \text { monolith covered with Ca- } \\
\text { montmorillonite }\end{array}$ & $\begin{array}{l}8 \text { wt } \% \mathrm{Fe}_{2} \mathrm{O}_{3}-15 \text { wt } \\
\% \mathrm{MnO}_{2}\end{array}$ & 1 & & & 1 & 860 & 97 & & & 473 \\
\hline $\begin{array}{l}\mathrm{TiO}_{2} \text { monolith covered with } \mathrm{Na}- \\
\text { montmorillonite }\end{array}$ & 5 wt $\% \mathrm{MnO}_{2}$ & 1 & & & 1 & 750 & 88 & & & 473 \\
\hline stainless steel microfibers & 10 wt $\% \mathrm{Ni} / \mathrm{Al}_{2} \mathrm{O}_{3}$ & 1 & 0.5 & 15 & 100 & 500 & 56 & & & 474 \\
\hline Ni microfibers & 10 wt $\% \mathrm{Ni} / \mathrm{Al}_{2} \mathrm{O}_{3}$ & 1 & 0.5 & 145 & 100 & 600 & 83 & & & 475 \\
\hline Ni microfibers & $\begin{array}{l}10 \text { wt } \% \text { Ni-10 wt } \% \\
\mathrm{Ce} / \mathrm{Al}_{2} \mathrm{O}_{3}\end{array}$ & 1 & 0.5 & 145 & 100 & 600 & 99 & & & 475 \\
\hline Ni microfibers & $\begin{array}{l}10 \text { wt } \% \mathrm{Ni}-10 \text { wt } \% \\
\mathrm{La} / \mathrm{Al}_{2} \mathrm{O}_{3}\end{array}$ & 1 & 0.5 & 145 & 100 & 600 & 93 & & & 475 \\
\hline
\end{tabular}

dehydrogenation of $\mathrm{NH}$ (eq 4) limits the reaction rate. From the analysis of the apparent activation energy, it was proposed that $\mathrm{Rh}$ was the most efficient catalyst among the three elements considered because it had the lowest apparent activation energy. Stolbov and Rahman ${ }^{427}$ analyzed the kinetics of the decomposition reaction on the $\mathrm{Ni}(111)$ and $\mathrm{Pd}(111)$ surfaces, concluding that ammonia dissociation is the limiting step for Pd. In a similar manner, Guo and Vlachos ${ }^{428}$ compared the kinetics of the reaction on $\mathrm{Ni}, \mathrm{Pt}$, and bimetallic $\mathrm{Ni}-\mathrm{Pt}$ catalysts, obtaining that $\mathrm{Ni}-\mathrm{Pt}$ should be a more active catalyst than $\mathrm{Pt}$ and $\mathrm{Ni}$ surfaces due to a lower ammonia dissociation energy barrier. Through the analysis of reaction kinetics of various $\mathrm{Ni}$ and $\mathrm{Pt}$ monometallic catalysts and $\mathrm{Ni}-\mathrm{Pt}$ and $\mathrm{Fe}-\mathrm{Pt}$ bimetallic catalysts, $\mathrm{Wu}$ et al. ${ }^{429}$ concluded that the bimetallic catalysts should be more active. By analyzing $\mathrm{Pt}, \mathrm{Ru}, \mathrm{Fe}$, and Re catalysts, Shustorovich and Bell ${ }^{430}$ determined that the recombinative desorption of nitrogen is the limiting step of the ammonia decomposition reaction, and that $\mathrm{Pt}$ is the most efficient catalyst, followed by $\mathrm{Ru}, \mathrm{Fe}$, and finally $\mathrm{Re}$. Although the apparent activation energy value calculated for $\mathrm{Cu}$ is very high, $317 \mathrm{~kJ}$ $\mathrm{mol}^{-1}$, it shows high activity when combined with $\mathrm{Pd}^{431}$ Mianowski et al. ${ }^{432}$ compared $\mathrm{Ni}, \mathrm{Pd}$, and $\mathrm{Pd}-\mathrm{Ni}$ catalysts supported on $\mathrm{SiO}_{2}$, obtaining the lowest activation energy with the bimetallic catalyst.

Srifa et al. ${ }^{350}$ determined that with the use of bimetallic catalysts made up of Mo combined with $\mathrm{Co}, \mathrm{Ni}$, and $\mathrm{Fe}$ in their nitride forms, the expression of the reaction rate does not depend on the concentration of $\mathrm{N}_{2}$, but it depends on that of $\mathrm{H}_{2}$. Moreover, the inhibition effect by hydrogen decreases compared to that of the catalyst composed only of molybdenum, which explains its higher catalytic activity. A kinetic study of the decomposition using a $\mathrm{Mo}_{3} \mathrm{~N}_{2}$ catalyst concluded that the determining step of the reaction in this case is the second dehydrogenation (eq 3), and that the catalyst has a higher 
apparent activation energy compared to $\mathrm{Ru}$ or $\mathrm{V}$ catalysts, confirming its lower catalytic activity. ${ }^{433}$

Okura et al. ${ }^{254}$ determined that, for Ni catalysts supported on rare earth (Y, La, Ce, Sm, Gd) and $\mathrm{Al}$ oxides, the catalytic activity is governed by the inhibition of hydrogen, the inhibition being particularly severe for $\mathrm{Ni} / \mathrm{CeO}_{2}$ and $\mathrm{Ni} / \mathrm{Al}_{2} \mathrm{O}_{3}$. A similar result was obtained by the same group, ${ }^{257}$ who compared the influence of the partial pressure of hydrogen on the reaction rate, obtaining that the catalyst that presents the best results among those of $\mathrm{Ni} / \mathrm{Al}_{2} \mathrm{O}_{3}$ promoted with rare earths (Y, La, Ce, $\mathrm{Pr}, \mathrm{Nd}$, $\mathrm{Sm}, \mathrm{Eu}$ and $\mathrm{Gd}$ ) is the one modified with $\mathrm{La}$, which is the one that is least inhibited by the presence of $\mathrm{H}_{2}$. Similarly, Zhang et al. ${ }^{251}$ compared $\mathrm{Ni} / \mathrm{Al}_{2} \mathrm{O}_{3}$ with $\mathrm{Ni} / \mathrm{La}-\mathrm{Al}_{2} \mathrm{O}_{3}$ and suggested that the best catalytic activity obtained by adding La to the support is due to its ability to destabilize the reaction intermediates on the active sites, and consequently facilitating their evolution. Sayas et al. ${ }^{73}$ observed that $\mathrm{K}$ allows a decrease in the $\mathrm{H}_{2}$ inhibition effect of a $\mathrm{Ru} / \mathrm{CaO}$ catalyst promoted with $\mathrm{K}$ with respect to the nonpromoted catalyst.

The ammonia decomposition using transition metal amides or imides follow a different reaction mechanism, where the functional group $\left(-\mathrm{NH}_{2}\right.$ or $\left.-\mathrm{NH}\right)$ participates. ${ }^{434}$ For example, with a $\mathrm{MnN}-\mathrm{Li}_{2} \mathrm{NH}$ catalyst, the desorption of $\mathrm{N}_{2}$, which was determined as the most commonly limiting step for nitrides or transition metals, appears to be fast, while the desorption of $\mathrm{H}_{2}$ is kinetically slow. ${ }^{379}$

Table 11 shows the catalysts used to study the kinetics of ammonia decomposition, the limiting step of the reaction, and the apparent activation energy ranges. A single limiting step has not been determined for most catalysts, which shows that the reaction mechanism does not depend only on the composition of the catalyst, but on many other factors, such as the synthesis method, the metal content, or the reaction conditions. Estimated apparent activation energy values for bimetallic catalysts are generally lower compared to monometallic ones. The lowest values of apparent activation energy are obtained in the case of $\mathrm{Ni}-\mathrm{Pt}$ and $\mathrm{Ni}-\mathrm{Pd}$, while the highest values of apparent activation energy are obtained for $\mathrm{Fe}, \mathrm{Cu}$, and $\mathrm{Mo}_{2} \mathrm{~N}$ catalysts that in turn have low catalytic activity.

3.3. Structured Systems. Since fixed-bed reactors with powder catalysts are not suitable for practical application, ${ }^{391,461}$ different structured catalytic reactors have been developed for the decomposition of ammonia. In particular, structured reactors with straight channels (honeycombs) and microreactors are the most common structured reactors developed up to date. ${ }^{46,148}$ Table 12 shows the types of structured systems described in the literature used to obtain hydrogen from the catalytic decomposition of ammonia along with the catalysts used, the reaction conditions, and the ammonia conversion or hydrogen production obtained. The apparent activation energy is also included when it was reported.

3.3.1. Types of Structured Systems. In some microreactors, the ammonia decomposition reaction was coupled with exothermic reactions, as discussed in section 2.1, in order to generate the heat necessary for the process and to achieve an autothermal regime, or close to it. Arana et al. ${ }^{103}$ designed a reactor composed of four microtubes of $\mathrm{SiN}_{x}$ with a wall thickness of $2 \mu \mathrm{m}$ coated with an $\mathrm{Ir} / \mathrm{Al}_{2} \mathrm{O}_{3}$ catalyst. In addition, the decomposition reaction of ammonia was coupled with the combustion of butane. Using a power of $1 \mathrm{~W}$ they were able to reach a temperature of $\sim 600{ }^{\circ} \mathrm{C}$ and an ammonia conversion of $12 \%$ at atmospheric pressure. Chen and $\operatorname{Yan}^{98}$ modeled a parallel plate microreactor with conductive walls with a thickness of $0.3 \mathrm{~mm}$ and channels $0.3 \mathrm{~mm}$ wide coated with a ruthenium catalyst, where the decomposition of ammonia was coupled with the combustion of methane. Similarly, Kaisare et al. ${ }^{104}$ created a model for an analogous catalytic reactor, with parallel plates with catalytic combustion of propane and ammonia decomposition channels that used a $\mathrm{Ru} / \mathrm{Al}_{2} \mathrm{O}_{3}$ catalyst. In a separate work they analyzed a perpendicular configuration of channels. ${ }^{105}$ Deshmukh and Vlachos ${ }^{106}$ considered propane combustion as an exothermic reaction in channels parallel to the decomposition of ammonia in a microreactor with channels $150 \mu \mathrm{m}$ wide and a wall thickness of $300 \mu \mathrm{m}$ using a $\mathrm{Ru}$ catalyst. They also created a model to study the best strategy for the thermal coupling of the two reactions. ${ }^{476}$ Engelbrecht et al. ${ }^{99}$ and Chiuta et al. ${ }^{100}$ coupled decomposition with ammonia oxidation in an autothermal parallel plate steel microreactor, using a $\mathrm{Ru} / \mathrm{Al}_{2} \mathrm{O}_{3}$ catalyst.

Another type of microreactor investigated for the decomposition of ammonia is the one containing geometrically distributed posts coated with a catalyst. A 3D-printed SiCN ceramic support with 139 pillars (Figure 6) was impregnated

(a)
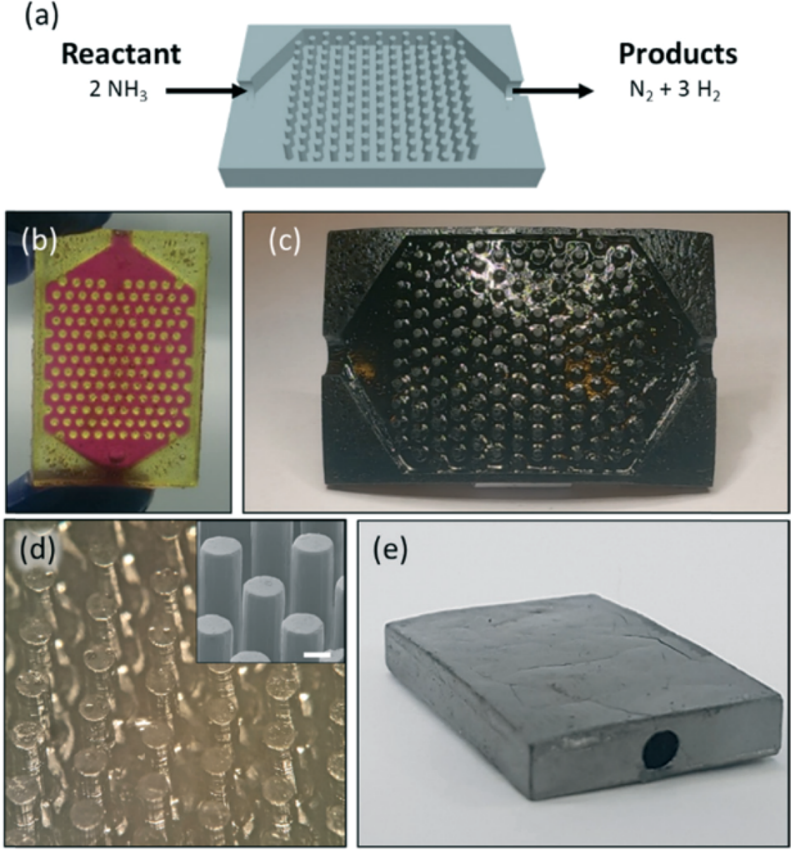

(e)

Figure 6. Catalytic microreactor designed for the ammonia decomposition reaction by Gyak et al. ${ }^{462}$ Reprinted with permission from ref 462. Copyright 2019 Royal Society of Chemistry.

with $\mathrm{Ru}$ and tested for the reaction, obtaining an ammonia conversion of $80 \%$ at $600{ }^{\circ} \mathrm{C} .{ }^{462}$ The microreactor maintained its integrity and functionality after prolonged exposure to ammonia at temperatures up to $1000{ }^{\circ} \mathrm{C}$ for $48 \mathrm{~h}$. Ganley et al. ${ }^{464,477}$ developed a similar post microreactor with $\mathrm{Ru} / \mathrm{Al}_{2} \mathrm{O}_{3}$ and compared its activity with various types of parallel microchannel microreactors with different geometries. The best conversion results were obtained with $140 \mu \mathrm{m}$ wide microchannels. It is important to mention that the promoting effect of $\mathrm{K}$ on $\mathrm{Ru} / \mathrm{Al}_{2} \mathrm{O}_{3}$ was also verified. In a similar manner and by preparing a ceramic microreactor composed of porous silicon carbide with microchannels impregnated with $\mathrm{Ru}$, Christian et al. ${ }^{465}$ obtained the best catalytic results with pores having a diameter of $0.75 \mu \mathrm{m}$ and a complete conversion at 500 ${ }^{\circ} \mathrm{C}$. Using the $3 \mathrm{D}$ printing technique, a microchannel structure 
made entirely of cerium dioxide was prepared and subsequently impregnated with nickel. The catalyst thus prepared showed a higher catalytic activity in the decomposition of ammonia than a conventional cordierite monolith washcoated with a catalyst with a similar composition $\left(\mathrm{Ni} / \mathrm{CeO}_{2}\right) .{ }^{463}$ Another type of stainless steel microchannel reactor loaded with a $\mathrm{Ni}-\mathrm{Pt} / \mathrm{Al}_{2} \mathrm{O}_{3}$ bimetallic catalyst deposited on $450 \mu \mathrm{m}$ wide channels was designed by Chiuta et al. ${ }^{466}$ The reactor showed good activity and stability in a $150 \mathrm{~h}$ test. Using the same microreactor but with a $\mathrm{Ru} / \mathrm{Al}_{2} \mathrm{O}_{3}$ catalyst promoted with $\mathrm{Cs}$, they obtained total ammonia conversion at $200{ }^{\circ} \mathrm{C}$ less than using $\mathrm{Ni}-\mathrm{Pt} / \mathrm{Al}_{2} \mathrm{O}_{3}$ and a good stability over time. ${ }^{467}$

Dillon et al. ${ }^{478}$ demonstrated by infrared spectroscopy that a silicon plate without catalyst is active in the decomposition of ammonia. Taking advantage of the Dillon results, Sørensen et al. $^{469}$ deposited alumina on a silicon plate and impregnated it with different metals, obtaining the following order of catalytic activity in the decomposition of ammonia: $\mathrm{Co}>\mathrm{Pd}>\mathrm{Ru}>\mathrm{Fe}$ at $575^{\circ} \mathrm{C}$. An increase in $\mathrm{H}_{2}$ production was obtained by adding $\mathrm{Ba}$ as a promoter of $\mathrm{Ru}$. Following this work, Sørensen et al. ${ }^{470}$ used a Ru catalyst without promoter or promoted with $\mathrm{Ba}$ and $\mathrm{Cs}$ in a graphite microreactor and obtained a higher catalytic activity in the case of $\mathrm{Ru}$ promoted with $\mathrm{Ba}$ with respect to the catalyst promoted with Cs or without promoters.

Regarding honeycomb structures, Plana et al. ${ }^{471}$ deposited a $\mathrm{Ni} / \mathrm{Al}_{2} \mathrm{O}_{3}$ catalyst on the walls of a cordierite monolith. By using a honeycomb configuration a higher ammonia conversion was obtained compared to a catalyst with the same composition but in a fixed bed reactor, although it is important to mention that the $\mathrm{Ni}$ content of the powdered catalyst used in the fixed bed reactor was around 4.5 times less by weight than the structured catalyst. They also tested the catalytic honeycomb after crushing it, and they observed a higher activity for the structured reactor, probably due to a more uniform reagent flow distribution. ${ }^{479}$ In a similar manner, Armenise et al. ${ }^{385}$ coated a cordierite honeycomb with $\mathrm{Al}_{2} \mathrm{O}_{3}$ and subsequently $\mathrm{Ru}$. A comparison of the conversion of ammonia with that obtained in the work described previously ${ }^{471}$ with the monolith prepared using the same method but with Ni showed that a higher conversion was obtained with $\mathrm{Ru}$ with respect to Ni. Subsequently, Armenise et $\mathrm{al}^{472}$ prepared and tested a cordierite honeycomb functionalized with nitrogen-doped carbon nanofibers, which served as support for $\mathrm{Ru}$, obtaining higher catalytic activity. Another type of honeycomb catalytic structures were prepared by Ismagilov et al., ${ }^{473}$ who deposited $\mathrm{MnO}_{2}$ on the walls of $\mathrm{TiO}_{2}$ honeycombs with $\mathrm{Ca}$ - or Na-montmorillonite as binder.

Liu et al. ${ }^{474}$ prepared stainless steel microfibers with alumina in their pores and impregnated with nickel, obtaining complete ammonia conversion at $650{ }^{\circ} \mathrm{C}$ with a high contact velocity. By using the same technique, $\mathrm{Ni} / \mathrm{Al}_{2} \mathrm{O}_{3}$ catalysts promoted with $\mathrm{Ce}$ or La supported on nickel microfibers were also prepared. ${ }^{475}$ The microfibers with the catalyst promoted with $\mathrm{Ce}$ had the highest catalytic activity, and the two promoted catalysts had a higher activity compared to the microfibers with the nonpromoted catalyst. Similarly, Wang et al. ${ }^{480}$ developed a $50 \mathrm{~cm}^{3}$ reactor with nickel microfibers and $\mathrm{Ni} / \mathrm{Al}_{2} \mathrm{O}_{3}$ promoted with $\mathrm{CeO}_{2}$, obtaining a complete ammonia conversion at $550^{\circ} \mathrm{C}$ and high stability in a test of $300 \mathrm{~h}$. Finally, Zou et al. ${ }^{481}$ proposed a cylindrical structured reactor composed of $\mathrm{MgO}$, to which small amounts of $\mathrm{CaCO}_{3}, \mathrm{MgCO}_{3}, \mathrm{TiO}_{2}$, activated carbon, and graphite were added, and subsequently impregnated with $\mathrm{Ni}$.

3.3.2. Modeling. A chemical reactor model is a suitable tool to understand the relationship between the catalytic properties and the geometry of a structure, and also to predict its catalytic behavior under different conditions. ${ }^{482} \mathrm{~A}$ model can also be classified as washcoat (it only provides information within the catalytic layer), channel (where one channel represents the entire reactor), or a scale model of the reactor. ${ }^{483}$ The choice of the scale of the model depends on the purpose of the application and the complexity of the model. In the literature, several mathematical models have been developed for the decomposition of ammonia using catalytic monoliths or microchannel structures, from $1 \mathrm{D}$ to 3D models at different scales.

Deshmukh et al. ${ }^{388}$ modeled the microreactor proposed by Ganley et al. ${ }^{477}$ using a 2D model. They studied the kinetics of the reaction and tested different types of models. Chen et al. ${ }^{484}$ created a model for a reactor loaded with $\mathrm{Ni}-\mathrm{Pt} / \mathrm{Al}_{2} \mathrm{O}_{3}$ and used it to optimize the reactor and the reaction conditions. Molaeimanesh and Davarani ${ }^{485}$ designed a 2D model to analyze the behavior of various types of microreactors equipped either with microfibers or posts and concluded that the best activity was achieved with microfiber microreactors. Armenise et al. ${ }^{386}$ used the Langmuir-Hinshelwood approach to analyze the kinetics of the cordierite honeycomb washcoated with $\mathrm{Ni} / \mathrm{Al}_{2} \mathrm{O}_{3}$ described previously ${ }^{471}$ for modeling purposes. In a similar manner, Chiuta et al. ${ }^{381}$ created a model for the microchannels loaded with $\mathrm{Ni}-\mathrm{Pt} / \mathrm{Al}_{2} \mathrm{O}_{3}$ presented previously. ${ }^{466}$ They considered a single microchannel as representative of the entire structure and optimized the design conditions taking into account the $\mathrm{NH}_{3}$ flow rate, the thickness of the catalyst layer, and the hydraulic diameter of the channel. They created a similar model with $\mathrm{Ru} / \mathrm{Al}_{2} \mathrm{O}_{3}$ promoted with $\mathrm{Cs}$ using the microchannels of the same stainless steel structure ${ }^{468}$ based on their previous experimental results. ${ }^{467}$ Using a $2 \mathrm{D}$ model of a single channel, Waghode et al. ${ }^{486}$ simulated the behavior of a microreactor loaded with a $\mathrm{Ni}$ catalyst for the generation of $\mathrm{H}_{2}$ from ammonia to feed a fuel cell and compared the results with those obtained with a fuel cell that directly uses ammonia. Similarly, Zade et al. ${ }^{487}$ used one channel as representative of the entire structure, and they designed a model for a $15 \mu \mathrm{m}$ wide microchannel loaded with a Ru catalyst, analyzing the effect of gas velocity, temperature, and reaction kinetics along the channel.

Models have also been developed to describe fixed bed reactors, considering mass and energy transfer issues. The models are useful to optimize the reaction conditions and the characteristics of the catalytic bed itself. For example, Badescu $^{488}$ designed a model of a fixed-bed reactor with a Ni/ $\mathrm{Al}_{2} \mathrm{O}_{3}$ catalyst in order to minimize the presence of ammonia at the outlet and the energy required to control the temperature in the reactor, controlling not only the reaction conditions, but also the geometry of the reactor. Moreover, Chein et al. ${ }^{489}$ designed a model for a $\mathrm{Ni}-\mathrm{Pt} / \mathrm{Al}_{2} \mathrm{O}_{3}$ catalyst to analyze the relationship between the decomposition of ammonia with the porosity and the size of the catalyst particles, as well as the effect of temperature, pressure, and ammonia flow rate, obtaining that a $1 \mathrm{D}$ plug flow model was a good approximation of the experimental results. Finally, Alagharu et al. ${ }^{490}$ simulated the behavior of a fixed bed reactor loaded with a Ni-Pt catalyst with the objective of generating $100 \mathrm{~W}$ of net power in a PEM-type fuel cell with the hydrogen produced.

3.4. Membrane Reactors. To obtain high purity hydrogen outflows, metallic membranes composed of palladium or its alloys and proton-conducting ceramic membranes are currently the most suitable materials due to their high hydrogen selectivity. These types of membranes can work in a wide 
Table 13. Studies Related to Membrane Reactors Used for Ammonia Decomposition

\begin{tabular}{|c|c|c|c|c|c|c|c|c|c|c|}
\hline membrane comp & catalyst & $\begin{array}{c}P \\
\text { (bar) }\end{array}$ & $\begin{array}{c}\text { WHSV } \\
\left(\mathrm{mL} \mathrm{g}^{-1} \mathrm{~h}^{-1}\right)\end{array}$ & $\begin{array}{l}\mathrm{NH}_{3} \text { flow } \\
\left(\mathrm{mL} \mathrm{min}^{-1}\right)\end{array}$ & $\begin{array}{l}\% \mathrm{NH}_{3} \\
\text { inlet flow }\end{array}$ & $\begin{array}{c}\mathrm{T} \\
\left({ }^{\circ} \mathrm{C}\right)\end{array}$ & $\begin{array}{c}\text { conv } \\
(\%)\end{array}$ & $\begin{array}{c}\text { conv (no } \\
\text { membrane) (\%) }\end{array}$ & $\begin{array}{c}E_{\mathrm{a}} \\
\left.(\mathrm{kJ} \mathrm{mol})^{-1}\right)\end{array}$ & ref \\
\hline $\mathrm{Ru} / \mathrm{YSZ}+\mathrm{Pd}$ & & 5 & & & 100 & 400 & 93 & 31 & 160 & 391 \\
\hline $\mathrm{Ru}-\mathrm{Cs} / \mathrm{YSZ}+\mathrm{Pd}$ & & 5 & & 61 & 100 & 400 & 98 & & 160 & 391 \\
\hline $\begin{array}{l}\mathrm{Pd}+\text { stainless steel support }+ \\
\mathrm{MnO}_{x}\end{array}$ & $\mathrm{Ru} / \mathrm{MgO}$ & 3 & 1950 & 47 & 100 & 400 & 100 & 91 & & 499 \\
\hline $\begin{array}{l}\mathrm{Pd}-\mathrm{Ag}+\text { stainless steel support } \\
\quad+\mathrm{MnO}_{x}\end{array}$ & $\mathrm{Ru} / \mathrm{MgO}$ & & & & & & & & & 499 \\
\hline $\mathrm{Pd}+\mathrm{Al}_{2} \mathrm{O}_{3}$ support $+\mathrm{MnO}_{x}$ & $\mathrm{Ru} / \mathrm{MgO}$ & & & & & & & & & 499 \\
\hline $\mathrm{Pd}+\mathrm{Al}_{2} \mathrm{O}_{3}$ support & $\mathrm{Ru} / \mathrm{MgO}$ & & & & & & & & & 499 \\
\hline $\mathrm{Ru} / \gamma-\mathrm{Al}_{2} \mathrm{O}_{3} / \alpha-\mathrm{Al}_{2} \mathrm{O}_{3}+$ silica & & 1 & & 10 & 100 & 450 & 95 & 45 & & $500-502$ \\
\hline $\begin{array}{l}\mathrm{Nd}_{5.5} \mathrm{Mo}_{0.5} \mathrm{~W}_{0.5} \mathrm{O}_{11.25-\delta} / \\
\quad \mathrm{Nd}_{5.5} \mathrm{Mo}_{0.5} \mathrm{~W}_{0.5} \mathrm{O}_{11.25-\delta}-\mathrm{Ni}\end{array}$ & & 1 & 27273 & 50 & 20 & 600 & 42 & 24 & & 503 \\
\hline Pd + ceramic support & $\mathrm{Ni} / \mathrm{La}-\mathrm{Al}_{2} \mathrm{O}_{3}$ & 3 & 2000 & & 100 & 475 & 100 & 91 & & 504 \\
\hline Pd + stainless steel support & $\mathrm{Ru}-\mathrm{Na} / \mathrm{AC}$ & 1 & 20000 & 100 & 10 & 367 & 100 & 76 & & 505 \\
\hline $\mathrm{Pd}$ & $\mathrm{Ru} / \mathrm{SiO}_{2}$ & 1 & & 10 & 100 & 450 & 87 & 73 & & 389 \\
\hline $\mathrm{Pd}+\mathrm{Al}_{2} \mathrm{O}_{3}$ ceramic support & $\mathrm{Ni} / \mathrm{Al}_{2} \mathrm{O}_{3}$ & 16 & 20060 & 410 & 0.33 & 550 & 79 & 17 & 230 & 506 \\
\hline $\mathrm{Pd}+$ metallic support & $\mathrm{Ru} / \mathrm{Al}_{2} \mathrm{O}_{3}$ & 6 & & 400 & 100 & 450 & 99 & & & 507 \\
\hline $\mathrm{Pd}+\mathrm{Al}_{2} \mathrm{O}_{3}$ support $+\mathrm{Ru}$ & & 1 & & 10 & 100 & 375 & 99 & & & 508 \\
\hline $\mathrm{Pd}-\mathrm{Ag}$ & & 1 & & 10 & 100 & 375 & 51 & & & 508 \\
\hline $\mathrm{Pd} / \gamma-\mathrm{Al}_{2} \mathrm{O}_{3} / \alpha-\mathrm{Al}_{2} \mathrm{O}_{3}$ & $\mathrm{Ni} / \mathrm{Al}_{2} \mathrm{O}_{3}$ & 3 & 100 & & 100 & 500 & 98 & 94 & 80 & 509 \\
\hline $\mathrm{Pd} / \alpha-\mathrm{Al}_{2} \mathrm{O}_{3}$ & $\mathrm{Ni} / \mathrm{Al}_{2} \mathrm{O}_{3}$ & 3 & 100 & & 100 & 500 & 97 & 94 & & 509 \\
\hline $\mathrm{Pd}-\mathrm{Ag}$ & $\mathrm{Ru} / \mathrm{Al}_{2} \mathrm{O}_{3}$ & 1 & & 25 & 100 & 500 & 96 & 57 & & 510 \\
\hline $\begin{array}{l}\mathrm{Pd}-\mathrm{Ag}+\mathrm{Al}_{2} \mathrm{O}_{3} \text { support }+ \\
\mathrm{Al}_{2} \mathrm{O}_{3}-\mathrm{YSZ}\end{array}$ & $\mathrm{Ru} / \mathrm{Al}_{2} \mathrm{O}_{3}$ & 4 & & 500 & 100 & 425 & 98 & 86 & & 512 \\
\hline
\end{tabular}

range of temperatures, between 300 and $700{ }^{\circ} \mathrm{C}$ for metals and from 600 to $900{ }^{\circ} \mathrm{C}$ for ceramics. ${ }^{491}$ In the case of the metallic membranes, to lower the cost, increase the permeability of hydrogen, and increase the robustness of the reactor, a stainless steel porous support for the membrane is frequently used. ${ }^{492}$ Hydrogen permeation through the membrane is generally a process assisted by an inert sweep gas on the permeate side of the membrane, such as $\mathrm{Ar}$ or $\mathrm{N}_{2}$. The sweep gas can be used cocurrent or counter-current with respect to the permeated hydrogen flow, and the two configurations can give different results. ${ }^{493,494}$ However, if an inert sweep gas is used the hydrogen stream is diluted, which is contrary to the objective pursued. The membranes can have a separation function, or catalytic properties for the reaction. ${ }^{495}$ When the membrane is surrounded by the catalyst, it is defined as a catalytic membrane reactor (CMR), while if the membrane is placed downstream of the catalytic reaction, it is referred to as a staged membrane reactor (SMR), where the separation occurs after the catalytic reaction. ${ }^{496}$ An important advantage of CMRs is that they shift the equilibrium toward hydrogen; this is the so-called "shift effect" and allows the process to proceed at lower temperature and with more compact reactors.

In the case of ammonia decomposition, various types of membrane reactors have been used, mainly CMRs-based. One of the main applications is the generation of pure hydrogen to feed low temperature PEM fuel cells without corrosion problems caused by the presence of ammonia. ${ }^{390}$ In a study developed by Lundin et al., ${ }^{497}$ the feasibility of using Pd membranes in the decomposition of ammonia was determined in terms of the effect of $\mathrm{N}_{2}$ and $\mathrm{NH}_{3}$ on the membrane. No inhibition of the hydrogen permeation was observed by exposure to pure $\mathrm{N}_{2}$ or $\mathrm{NH}_{3}$ or $\mathrm{H}_{2} / \mathrm{N}_{2}$ and $\mathrm{H}_{2} / \mathrm{NH}_{3}$ mixtures for pressures up to $10 \mathrm{bar}$ and temperatures in the range of 325 to $500{ }^{\circ} \mathrm{C}$. A similar analysis was carried out by Sakamoto et al. $^{498}$ using various types of Pd alloy membranes. The experimental studies performed with membrane reactors for ammonia decomposition are compiled in Table 13, where the reaction conditions, the conversion obtained in comparison with the configuration without a membrane, and the apparent activation energy in the cases where it has been determined, are presented. There are two types of techniques used to create the necessary pressure difference between the two sides of the membrane (retentate and permeate sides): elevated pressure on the retentate side and atmospheric in the permeate, or low pressure on the retentate side and vacuum in the permeate. For obvious reasons, the first configuration is preferred in practical applications, especially considering that fuel cells operate at pressures slightly above atmospheric.

Most of the studies have used Pd or Pd-M ( $\mathrm{M}=\mathrm{Ag}, \mathrm{Cu})$ membranes supported on a metal (usually porous stainless steel) or a ceramic support (especially $\mathrm{Al}_{2} \mathrm{O}_{3}$ ). Nevertheless, novel membrane compositions have also been studied, such as that reported by Zhang et al., ${ }^{391}$ who prepared a tube of yttria stabilized zirconia impregnated with $\mathrm{Ru}$ where a layer of Pd was deposited as a membrane. They also analyzed the consequences on the catalytic activity of the addition of a Cs promoter, which led to complete ammonia conversion at temperatures as low as $400{ }^{\circ} \mathrm{C}$, exceeding equilibrium restrictions without using sweep gas.

The modification of the two types of support, steel and ceramic, with other compounds, such as $\mathrm{MnO}_{x}$, has also been investigated. For instance, Liu et al. ${ }^{499}$ prepared various types of membrane reactors, using $\mathrm{Pd}$ or $\mathrm{Pd}-\mathrm{Ag}$ supported on stainless steel or $\mathrm{Al}_{2} \mathrm{O}_{3}$. Both supports were modified with $\mathrm{MnO}_{x}$, to create an intermediate layer between the support and the membrane. Using a $\mathrm{Ru} / \mathrm{MgO}$ catalyst they obtained good results for the ammonia decomposition, especially with the $\mathrm{Pd}$ membrane supported on porous stainless steel modified with $\mathrm{MnO}_{x}$, with which they obtained good stability after a $200 \mathrm{~h}$ run at $400{ }^{\circ} \mathrm{C}$. Cechetto et al. ${ }^{512}$ recently performed the reaction in a $\mathrm{Pd}-\mathrm{Ag}$ membrane with an $\mathrm{Al}_{2} \mathrm{O}_{3}$ support, adding to the selective layer a protective layer of porous $\mathrm{Al}_{2} \mathrm{O}_{3}$ with yttria- 


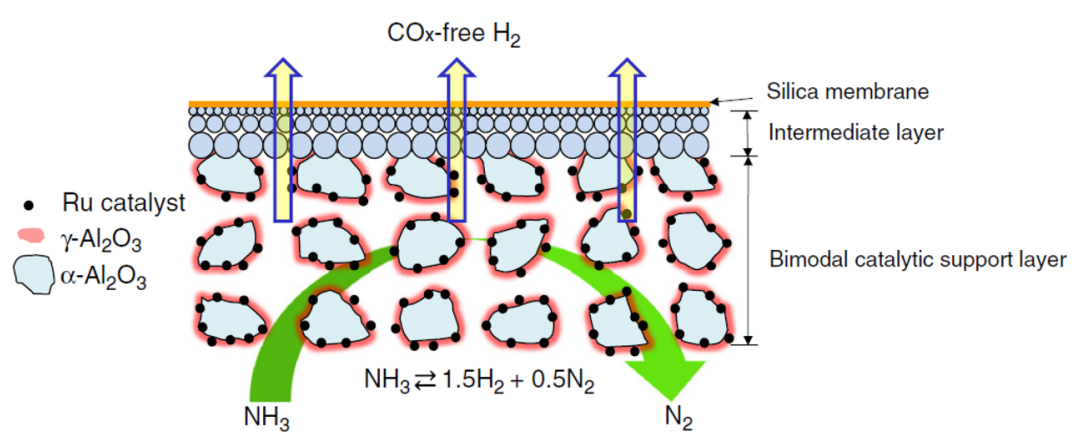

Figure 7. Scheme of the catalytic membrane used by Li et al. ${ }^{501}$ Reprinted with permission from ref 501. Copyright 2011 Elsevier.

stabilized zirconia to improve the membrane stability. They used a $\mathrm{Ru}$-based catalyst supported on $\mathrm{Al}_{2} \mathrm{O}_{3}$ pellets, and they compared ammonia conversion and membrane recovery efficiency with 1 bar of pressure on the permeate side, or by applying vacuum. A higher $\mathrm{H}_{2}$ recovery and, consequently, faster kinetics and conversion, was obtained when vacuum was applied.

Other types of membranes used are silica ${ }^{500}$ or materials composed of $\mathrm{Nd}, \mathrm{Mo}$, and $\mathrm{W} .{ }^{503} \mathrm{Li}$ et al. ${ }^{501}$ tested a catalytic membrane reactor loaded with $\mathrm{Ru} / \gamma-\mathrm{Al}_{2} \mathrm{O}_{3} / \alpha-\mathrm{Al}_{2} \mathrm{O}_{3}$ and a hydrogen-selective silica membrane (Figure 7). The conversion of $\mathrm{NH}_{3}$ in the presence of the membrane increases significantly, from $45 \%$ to $95 \%$ at $450{ }^{\circ} \mathrm{C}$, as a consequence of the shift effect due to the extraction of $\mathrm{H}_{2}$. ${ }^{502}$

Using a different approach, Cheng et al. ${ }^{503}$ used a double layered membrane $\mathrm{Nd}_{5.5} \mathrm{Mo}_{0.5} \mathrm{~W}_{0.5} \mathrm{O}_{11.25-\delta} /$ $\mathrm{Nd}_{5.5} \mathrm{Mo}_{0.5} \mathrm{~W}_{0.5} \mathrm{O}_{11.25-\delta}-\mathrm{Ni}$ that maintained a very high $\mathrm{NH}_{3}$ conversion and $\mathrm{H}_{2}$ permeation flux, stable during a continuous test of 75 h at $750{ }^{\circ} \mathrm{C}$.

Regarding the catalysts, most of the studies have used conventional $\mathrm{Ru}$ or $\mathrm{Ni}$ supported on alumina, while in other works alternative catalysts have been tested, for example promoted with $\mathrm{La},{ }^{504}$ or supported on carbon, ${ }^{505} \mathrm{SiO}_{2}{ }^{389}$ or MgO. ${ }^{499}$ In this sense, Zhang et al. ${ }^{504}$ used a Ni/La- $\mathrm{Al}_{2} \mathrm{O}_{3}$ catalyst with a membrane of $\mathrm{Pd}$ and ceramic material $\left(\mathrm{Al}_{2} \mathrm{O}_{3}\right)$ supported on stainless steel. They were able to determine an optimal catalytic activity and stability of the reactor during a 500 $\mathrm{h}$ test. Residual ammonia slightly inhibited the permeability of the membrane, but through a treatment with air followed by $\mathrm{H}_{2}$ the permeability could be recovered. With a $\mathrm{Pd}$ membrane supported on porous stainless steel and a $\mathrm{Ru} / \mathrm{C}$ catalyst promoted with $\mathrm{Na}$, complete ammonia conversion was achieved at $367{ }^{\circ} \mathrm{C}$, exceeding the corresponding thermodynamic equilibrium conversion by approximately $2 \%{ }^{505}$ Collins et al. $^{506}$ analyzed the conversion of a small amount of ammonia (0.33 mol \%) on $\mathrm{Ni} / \mathrm{Al}_{2} \mathrm{O}_{3}$ for gas cleaning, obtaining a conversion of $79 \%$ at $450{ }^{\circ} \mathrm{C}$ using a $\mathrm{Pd}$ membrane on an $\mathrm{Al}_{2} \mathrm{O}_{3}$ ceramic support. This conversion is $62 \%$ higher than with the same catalyst in a conventional reactor.

Itoh et al. ${ }^{389}$ used a $\mathrm{Ru} / \mathrm{SiO}_{2}$ catalyst with a $200 \mu \mathrm{m}$ thick palladium membrane and obtained a $15 \%$ increase in conversion compared to the conventional reactor, and $60 \%$ of hydrogen recovery at $450{ }^{\circ} \mathrm{C}$. Similarly, a ruthenium catalyst supported on alumina was tested in pellet form by Rizzuto et al. in a CMR equipped with a $\mathrm{Pd}$ membrane, ${ }^{507}$ obtaining a complete conversion with a space velocity of $653 \mathrm{~h}^{-1}$ at $450{ }^{\circ} \mathrm{C}$ and 6 bar. Also, Itoh et al. ${ }^{508}$ investigated a reactor with a $2 \mu \mathrm{m}$ thick $\mathrm{Pd}$ membrane deposited on an $\mathrm{Al}_{2} \mathrm{O}_{3}$ tube and impregnated with $\mathrm{Ru}$, and compared the catalytic results with an $\mathrm{Ag}-\mathrm{Pd}$ membrane, obtaining a significantly higher $\mathrm{NH}_{3}$ conversion with the first configuration, reaching complete conversion at 375 ${ }^{\circ} \mathrm{C}$ with a reduced flow of pure ammonia.

Some studies have used the experimental results to build a model, and use it to simulate different reaction conditions to optimize the CMR reactor. For example, using a Ni/ $\mathrm{Al}_{2} \mathrm{O}_{3}$ catalyst and a $4 \mu \mathrm{m}$ Pd membrane supported and encapsulated over two $\mathrm{Al}_{2} \mathrm{O}_{3}$ phases, Israni et al. ${ }^{509}$ created a model to describe the behavior of the membrane reactor and compare it with that of a Pd membrane reactor and a conventional $13 \mu \mathrm{m}$ $\mathrm{Al}_{2} \mathrm{O}_{3}$ support. Using the same approach, Prasad et al. ${ }^{510}$ designed a model for a CMR reactor with a $\mathrm{Pd}-\mathrm{Ag}$ membrane and a $\mathrm{Ru} / \mathrm{Al}_{2} \mathrm{O}_{3}$ catalyst and experimentally confirmed the results, obtaining complete conversion at lower temperatures compared to the reactor without a membrane.

Without confirming the results experimentally, Abashar ${ }^{387,392}$ simulated the behavior of a $\mathrm{Pd}-\mathrm{Ag}$ membrane reactor coupled with a pellet catalyst through a model, and concluded that using more than one reactor, that is, a multistage configuration, would have significant advantages in terms of ammonia decomposition. By using a single stage they predicted an ammonia conversion of around $29 \%$ at 40 bar, while with seven stages the conversion becomes complete. Di Carlo et al. ${ }^{511}$ designed a reactor with more than one $\mathrm{Pd}$ membrane inside it and a Ni/ $\mathrm{Al}_{2} \mathrm{O}_{3}$ catalyst, and optimized the reaction conditions through a model using seven membranes, a temperature of $550^{\circ} \mathrm{C}$ and a pressure of 10 bar. Another work which focused on analyzing the performance of a reactor with 19 vanadium-based alloy membrane tubes was carried out by Hla and Dolan, ${ }^{513}$ who obtained a very high hydrogen yield at a low ammonia inlet flow and a reaction pressure of 7.8 bar.

\section{SUMMARY AND CONCLUSIONS}

Ammonia decomposition is a well-known reaction that was first used essentially for fundamental studies. With the imminent arrival of the energy transition, hydrogen technologies are gaining relevance, and the generation of hydrogen from the decomposition reaction of ammonia represents an interesting route to produce carbon-free hydrogen on-site and on-demand. Ammonia can be considered as a hydrogen storage system, as it is liquid at low pressure at ambient temperature, which means a high hydrogen density both in volume and weight. For this reason, numerous efforts have been devoted to the development of highly active and robust catalysts, aimed at the decomposition of ammonia at the lowest possible temperature. The most studied catalysts contain a metallic function (frequently $\mathrm{Ni}$ or $\mathrm{Ru}$ ) supported on an inorganic oxide and modified by different types of promoters, usually alkaline. It is well established that ruthenium is the most active metal for the decomposition of 
ammonia, as it allows to obtain the highest conversion at the lowest temperature, of the order of $400{ }^{\circ} \mathrm{C}$, while Ni-based catalysts yield similar results, but at temperatures between 500 and $600{ }^{\circ} \mathrm{C}$. It should be noted, however, that a higher $\mathrm{Ni}$ content can be used due to its lower cost. Because of the high price of ruthenium, alternative systems based on easily obtainable non-noble metals are being investigated, generally composed of elements such as iron, cobalt, or molybdenum, even if they currently do not reach the activity of rutheniumbased catalysts. However, it should be noted that many of these inexpensive catalytic systems have not yet been studied as extensively as ruthenium or nickel and therefore it may be a lot of room for improvement. Nitrides and carbides of transition metals also show catalytic activity for the decomposition of ammonia; however, at present the catalytic activities that have been obtained are far from being considered an alternative to noble metals. Using theoretical models it has been predicted that bimetallic catalysts, such as Co-Mo, are suitable cost-effective candidates, but bimetallic systems are complex and an extensive characterization is needed to fully understand the nature of the active sites involved under reaction conditions to predict a proper combination of metals. ${ }^{514}$ The combinations tested so far confirm that bimetallic catalysts are more promising than monometallic ones. The investigation of novel compounds based on amide and imide of transition metals has recently started, and these materials already show remarkable potential as future catalysts for low temperature ammonia decomposition, especially when coupled with other active metals and nitrides, or used in a supported form.

The active phase, the support, and the promoters all play a determinant role in the reaction. It is generally accepted that for $\mathrm{NH}_{3}$ decomposition a catalyst support should present a high basicity, along with high conductivity, low concentration of electron-withdrawing groups, high thermal stability, and high surface area. In particular, many studies have related the activity of catalyst to basicity, and this has been reported for different active phases such as $\mathrm{Ru}, \mathrm{Ni}, \mathrm{Fe}, \mathrm{Co}$, and Mo. Basicity has been related to an increase in the dispersion of the active metal, but also to an enhancement for ammonia dehydrogenation and for the recombinative desorption of surface $\mathrm{N}$ atoms, which are the most likely rate-limiting steps of the reaction. Generally, the basicity of the catalyst is modulated by adding a promoter, which also allows to increase the electrodonating properties of the catalyst, since the promoters can have an indirect interaction with the support to induce stronger basicity. Although, in general, the relationship between the electrodonating properties of the promoter and the activity of the catalyst has been determined, the effect can be different depending on the active phase used due to direct electronic interaction with the active metal. For example, in the case of ruthenium, the alkali metals have proven to be good promoters, while in the case of cobalt or amides/imides the activity decreases. In the case of nickel, rare earths such as $\mathrm{Ce}$ and La have shown to be better promoters than alkali metals. In summary, the combination of properties such as basicity and a high electrodonation capacity of the support and promoters seems to be fundamental for the development of efficient catalysts for $\mathrm{NH}_{3}$ decomposition.

The development of these catalysts has been accompanied by the design of compact and efficient reactors in order to keep the reactor volume low for small and medium applications. In addition, structured reactors are superior to fixed-bed reactors due to their portability, superior heat and mass transfer capacity, and uniform flow distribution. In particular, microchannel reactors are most likely the structure with the highest potential for ammonia decomposition compared to conventional honeycomb structures and other types of structured reactors.

Kinetic studies and reactor modeling are useful and necessary tools for a better understanding of the reaction mechanism and for a rational design of catalytic devices. Many studies have focused on determining the limiting step of the decomposition reaction using different types of catalysts. Although initially it was proposed that the limiting step was the desorption of nitrogen from the surface of the catalyst and that, therefore, the binding energy with nitrogen could determine the activity of the catalyst, it was later seen that for various types of catalysts the limiting step was different. It has been proposed that for noble metals the limiting step is the cleavage of $\mathrm{N}-\mathrm{H}$ bonds, while that for non-noble metals it is the desorption of nitrogen. However, this issue is more complex since the limiting step for a given catalyst can vary with reaction conditions. Another important element that kinetic studies have focused on is the inhibition of hydrogen generated on the catalyst; in fact, it has been proposed that it is the inhibitory effect of hydrogen that governs catalytic activity. The extent of inhibition has been determined to vary with temperature and is greater at low temperatures due to hydrogen accumulation on the catalyst surface. For catalysts composed of $\mathrm{Ni}, \mathrm{Pt}$, or $\mathrm{Mo}$, it is found that the inhibition is stronger than that of other metals such as $\mathrm{Ru}$ or $\mathrm{Fe}$, and some promoters decrease the $\mathrm{H}_{2}$ inhibitory effect, such as $\mathrm{K}$ for $\mathrm{Ru}$ catalysts. Bimetallic formulations also decrease hydrogen inhibition.

Another method that makes it possible to reduce the inhibitory effect of hydrogen on the catalyst is the use of a selective membrane to remove it while it is being generated. Furthermore, the use of a catalytic membrane reactor implies a change in the reaction equilibrium (shift effect) and, therefore, an increase in the hydrogen yield. Also, when hydrogen is intended to power low-temperature fuel cells to produce electricity, as in PEM-type fuel cells, catalytic membrane reactors appear as the ideal solution to obtain a stream of pure hydrogen from ammonia decomposition. The only drawback is the current high price of Pd-based dense metallic membranes. A proper balance between catalyst characteristics and membrane properties is necessary for optimized performance.

While it is true that studies related to catalysts are at a very advanced stage, the development of practical catalytic systems for use in specific applications still requires great efforts. The optimization of the ammonia decomposition reaction for its application in a real hydrogen production system must be comprehensive and consider not only the type of catalyst, but also the type of reactor to allow the realization of a totally carbon-free energy technology.

\section{AUTHOR INFORMATION}

\section{Corresponding Author}

Jordi Llorca - Institute of Energy Technologies, Department of Chemical Engineering and Barcelona Research Center in Multiscale Science and Engineering, Universitat Politènica de Catalunya, Barcelona 08019, Spain; 이잉.org/00000002-7447-9582; Phone: +34 934011708;

Email: jordi.llorca@upc.edu

\section{Authors}

Ilaria Lucentini - Institute of Energy Technologies, Department of Chemical Engineering and Barcelona Research Center in 
Multiscale Science and Engineering, Universitat Politècnica de Catalunya, Barcelona 08019, Spain

Xènia Garcia - Institute of Energy Technologies, Department of Chemical Engineering and Barcelona Research Center in Multiscale Science and Engineering, Universitat Politècnica de Catalunya, Barcelona 08019, Spain

Xavier Vendrell - Institute of Energy Technologies, Department of Chemical Engineering and Barcelona Research Center in Multiscale Science and Engineering, Universitat Politècnica de Catalunya, Barcelona 08019, Spain

Complete contact information is available at:

https://pubs.acs.org/10.1021/acs.iecr.1c00843

\section{Notes}

The authors declare no competing financial interest.

\section{ACKNOWLEDGMENTS}

This work was supported by projects MICINN/FEDER RTI2018-093996-B-C31 and GC 2017 SGR 128. I.L. is grateful to MINECO for Ph.D. Grant BES-2016-076507. X.G. is grateful for a FI-DGR 2016 grant. X.V. is grateful to MICIU-Juan de la Cierva-Incorporación program for the individual fellowship grant agreement IJCI-2017-31449. J.L. is a Serra Húnter Fellow and is grateful to the ICREA Academia program.

\section{ACRONYMS AND ABBREVIATIONS}

AC activated carbon

AFC alkaline fuel cell

BHA barium hexaaluminate

CMFs carbon microfibers

CMR catalytic membrane reactor

CNFs carbon nanofibers

CNTs carbon nanotubes

conv conversion (in tables)

comp composition (in tables)

DBD dielectric barrier discharges

DMFC direct methanol fuel cell

$E_{\mathrm{a}} \quad$ apparent activation energy

GC graphitized carbon

GHSV gas hourly space velocity

GNP graphene nanoplatelet

GO graphene oxide

GWP global warming power

HNTs halloysite nanotubes

LHHW Langmuir-Hinshelwood-Hougen-Watson

MCFC molten carbonate fuel cell

MOFs metal-organic frameworks

MSC microporous superactivated carbon

MW multiwalled

NT nanotube

OMC ordered mesoporous carbon

$P \quad$ pressure

PAFC phosphoric acid fuel cell

PEMFC proton exchange membrane fuel cell

RGO reduced graphene oxide

SMR staged membrane reactor

SOFC solid oxide fuel cell

SW single-walled

$T$ temperature

TMN transition metal nitrides

TOF turn over frequency

WHSV weight hourly space velocity wt

YSZ yttria-stabilized zirconia

$\Delta H^{\circ} \quad$ standard enthalpy of reaction

\section{REFERENCES}

(1) York, R.; Bell, S. E. Energy Transitions or Additions? Why a Transition from Fossil Fuels Requires More than the Growth of Renewable Energy. Energy Res. Soc. Sci. 2019, 51, 40-43.

(2) Braff, W. A.; Mueller, J. M.; Trancik, J. E. Value of Storage Technologies for Wind and Solar Energy. Nat. Clim. Change 2016, 6 (10), 964-969.

(3) Markard, J. The next Phase of the Energy Transition and Its Implications for Research and Policy. Nat. Energy 2018, 3 (8), 628633.

(4) Bockris, J. O. M. The Hydrogen Economy: Its History. Int. J. Hydrogen Energy 2013, 38 (6), 2579-2588.

(5) Staffell, I.; Scamman, D.; Velazquez Abad, A.; Balcombe, P.; Dodds, P. E.; Ekins, P.; Shah, N.; Ward, K. R. The Role of Hydrogen and Fuel Cells in the Global Energy System. Energy Environ. Sci. 2019, 12 (2), 463-491.

(6) Pandev, M.; Lucchese, P.; Mansilla, C.; Le Duigou, A.; Abrashev, B.; Vladikova, D. Hydrogen Economy: The Future for a Sustainable and Green Society. Bulg. Chem. Commun. 2017, 49 (C), 84-92.

(7) Navarro, R. M.; Peña, M. A.; Fierro, J. L. G. Hydrogen Production Reactions from Carbon Feedstocks: Fossil Fuels and Biomass. Chem. Rev. 2007, 107 (10), 3952-3991.

(8) Kalamaras, C. M.; Efstathiou, A. M. Hydrogen Production Technologies: Current State and Future Developments. Conf. Pap. Energy 2013, 2013, 1-9.

(9) Crabtree, G. W.; Dresselhaus, M. S.; Buchanan, M. V. The Hydrogen Economy. Phys. Today 2004, 57 (12), 39-45.

(10) Ursúa, A.; Gandía, L. M.; Sanchis, P. Hydrogen Production from Water Electrolysis: Current Status and Future Trends. Proc. IEEE 2012, 100 (2), 410-426.

(11) International Renewable Energy Agency (IRENA). Hydrogen: A Renewable Energy Perspective; IRENA, 2019.

(12) International Energy Agency (IEA). Future of Hydrogen; IEA, 2019.

(13) Yacobucci, B. D.; Curtright, A. E. A Hydrogen Economy and Fuel Cells: An Overview; Congressional Resource Service, 2004.

(14) White, C. M.; Steeper, R. R.; Lutz, A. E. The Hydrogen-Fueled Internal Combustion Engine: A Technical Review. Int. J. Hydrogen Energy 2006, 31 (10), 1292-1305.

(15) van den Berg, A. W. C.; Otero Areán, C. Materials for Hydrogen Storage: Current Research Trends and Perspectives. Chem. Commun. 2008, 6, 668-681.

(16) Robertson, I. M.; Sofronis, P.; Nagao, A.; Martin, M. L.; Wang, S.; Gross, D. W.; Nygren, K. E. Hydrogen Embrittlement Understood. Metall. Mater. Trans. A 2015, 46 (6), 1085-1103.

(17) Zheng, J.; Liu, X.; Xu, P.; Liu, P.; Zhao, Y.; Yang, J. Development of High Pressure Gaseous Hydrogen Storage Technologies. Int. J. Hydrogen Energy 2012, 37 (1), 1048-1057.

(18) Züttel, A. Hydrogen Storage Methods. Naturwissenschaften 2004, 91 (4), 157-172.

(19) Ahluwalia, R. K.; Hua, T. Q.; Peng, J.-K.; Lasher, S.; McKenney, K.; Sinha, J.; Gardiner, M. Technical Assessment of Cryo-Compressed Hydrogen Storage Tank Systems for Automotive Applications. Int. J. Hydrogen Energy 2010, 35 (9), 4171-4184.

(20) Hwang, H. T.; Varma, A. Hydrogen Storage for Fuel Cell Vehicles. Curr. Opin. Chem. Eng. 2014, 5, 42-48.

(21) Subrahmanyam, K. S.; Kumar, P.; Maitra, U.; Govindaraj, A.; Hembram, K. P. S. S.; Waghmare, U. V.; Rao, C. N. R. Chemical Storage of Hydrogen in Few-Layer Graphene. Proc. Natl. Acad. Sci. U. S. A. 2011, 108 (7), 2674-2677.

(22) Nijkamp, M. G.; Raaymakers, J. E. M. J.; van Dillen, A. J.; de Jong, K. P. Hydrogen Storage Using Physisorption-Materials Demands. Appl. Phys. A: Mater. Sci. Process. 2001, 72, 619-623. 
(23) Sakintuna, B.; Lamari-Darkrim, F.; Hirscher, M. Metal Hydride Materials for Solid Hydrogen Storage: A Review. Int. J. Hydrogen Energy 2007, 32 (9), 1121-1140.

(24) Suh, M. P.; Park, H. J.; Prasad, T. K.; Lim, D.-W. Hydrogen Storage in Metal-Organic Frameworks. Chem. Rev. 2012, 112, 782835.

(25) Rowsell, J. L. C.; Yaghi, O. M. Strategies for Hydrogen Storage in Metal-Organic Frameworks. Angew. Chem., Int. Ed. 2005, 44 (30), 4670-4679.

(26) Murray, L. J.; Dincă, M.; Long, J. R. Hydrogen Storage in MetalOrganic Frameworks. Chem. Soc. Rev. 2009, 38 (5), 1294.

(27) Jensen, J. O.; Li, Q.; Bjerrum, N. J.; Palm, J. The Energy Efficiency of Onboard Hydrogen Storage Techniques. Energy Efficiency 2010, 143-156, DOI: 10.1016/j.jallcom.2007.04.051.

(28) Bossel, U.; Eliasson, B. Energy and the Hydrogen Economy. Eur. Fuel Cell Forum, Lucerne 2002, 1-36.

(29) Kojima, Y.; Suzuki, K.-I.; Fukumoto, K.; Sasaki, M.; Yamamoto, T.; Kawai, Y.; Hayashi, H. Hydrogen Generation Using Sodium Borohydride Solution and Metal Catalyst Coated on Metal Oxide. Int. J. Hydrogen Energy 2002, 27 (10), 1029-1034.

(30) Biniwale, R. B.; Rayalu, S.; Devotta, S.; Ichikawa, M. Chemical Hydrides: A Solution to High Capacity Hydrogen Storage and Supply. Int. J. Hydrogen Energy 2008, 33 (1), 360-365.

(31) Eberle, U.; Felderhoff, M.; Schüth, F. Chemical and Physical Solutions for Hydrogen Storage. Angew. Chem., Int. Ed. 2009, 48 (36), 6608-6630.

(32) Makepeace, J. W.; Wood, T. J.; Hunter, H. M. A.; Jones, M. O.; David, W. I. F. Ammonia Decomposition Catalysis Using NonStoichiometric Lithium Imide. Chem. Sci. 2015, 6 (7), 3805-3815.

(33) Lan, R.; Irvine, J. T. S.; Tao, S. Ammonia and Related Chemicals as Potential Indirect Hydrogen Storage Materials. Int. J. Hydrogen Energy 2012, 37 (2), 1482-1494.

(34) Klerke, A.; Christensen, C. H.; Nørskov, J. K.; Vegge, T. Ammonia for Hydrogen Storage: Challenges and Opportunities. J. Mater. Chem. 2008, 18 (20), 2304-2310.

(35) Zamfirescu, C.; Dincer, I. Using Ammonia as a Sustainable Fuel. J. Power Sources 2008, 185 (1), 459-465.

(36) Giddey, S.; Badwal, S. P. S.; Munnings, C.; Dolan, M. Ammonia as a Renewable Energy Transportation Media. ACS Sustainable Chem. Eng. 2017, 5 (11), 10231-10239.

(37) Lipman, T.; Shah, N. Ammonia as an Alternative Energy Storage Medium for Hydrogen Fuel Cells: Scientific and Technical Review for NearTerm Stationary Power Demonstration Projects; Transportation Sustainability Research Center, 2007.

(38) Venkat, P.; Richardson, J. Introduction to Ammonia Production. CEP Mag. 2016, No. 2, 69-75.

(39) Appl, M. Ammonia, 2. Production Processes. Ullmann's Encyclopedia of industrial chemistry 2011, 3, 1-88.

(40) Barona, A.; Etxebarria, B.; Aleksanyan, A.; Gallastegui, G.; Rojo, N.; Diaz-Tena, E. A Unique Historical Case to Understand the Present Sustainable Development. Sci. Eng. Ethics 2018, 24 (1), 261-274.

(41) The Royal Society. Ammonia: Zero-Carbon Fertiliser, Fuel and Energy Store. Policy Briefing; The Royal Society, 2020.

(42) Maruti Co. Ammonia Cracker; 2013, https://www. exportersindia.com/maruti-corporation-company5789622/ammoniacracker-4133077.htm (accessed 2021).

(43) Rouwenhorst, K. H. R.; Van der Ham, A. G. J.; Mul, G.; Kersten, S. R. A. Islanded Ammonia Power Systems: Technology Review \& Conceptual Process Design. Renewable Sustainable Energy Rev. 2019, 114, 109339.

(44) Lamb, K. E.; Dolan, M. D.; Kennedy, D. F. Ammonia for Hydrogen Storage; A Review of Catalytic Ammonia Decomposition and Hydrogen Separation and Purification. Int. J. Hydrogen Energy 2019, 44 (7), 3580-3593.

(45) Hejze, T.; Besenhard, J. O.; Kordesch, K.; Cifrain, M.; Aronsson, R. R. Current Status of Combined Systems Using Alkaline Fuel Cells and Ammonia as a Hydrogen Carrier. J. Power Sources 2008, 176 (2), 490-493.
(46) Chiuta, S.; Everson, R. C.; Neomagus, H. W. J. P.; Van Der Gryp, P.; Bessarabov, D. G. Reactor Technology Options for Distributed Hydrogen Generation via Ammonia Decomposition: A Review. Int. J. Hydrogen Energy 2013, 38 (35), 14968-14991.

(47) Valera-Medina, A.; Xiao, H.; Owen-Jones, M.; David, W. I. F.; Bowen, P. J. Ammonia for Power. Prog. Energy Combust. Sci. 2018, 69, 63-102.

(48) Zamfirescu, C.; Dincer, I. Ammonia as a Green Fuel and Hydrogen Source for Vehicular Applications. Fuel Process. Technol. 2009, 90 (5), 729-737.

(49) Ibrahim, O. M.; Klein, S. A. Absorption Power Cycles. Energy 1996, 21 (1), 21-27.

(50) Arias, J. Hydrogen and Fuel Cells in Japan; EU-Japan Centre for Industrial Cooperation, 2019.

(51) Kroch, E. Ammonia-A Fuel for Motor Buses. J. Inst. Pet. 1945, 31, 213-223.

(52) Siemer, D. Nuclear Power: Policies, Practices, and the Future; Wiley, 2019.

(53) Comotti, M.; Frigo, S. Hydrogen Generation System for Ammonia-Hydrogen Fuelled Internal Combustion Engines. Int. J. Hydrogen Energy 2015, 40 (33), 10673-10686.

(54) Hacker, V.; Kordesch, K. Ammonia Crackers. Handbook of Fuel Cells - Fundamentals, Technology and Applications 2003, 3, 121-127.

(55) Intelligent Energy. Fuel-Flexible Hestia Hydrogen-Generating System Demonstrated. Fuel Cells Bull. 2006, 2006 (8), 6 DOI: 10.1016/ S1464-2859(06)71153-1.

(56) Compact Microchannel Ammonia Crackers. https:// pocharitechnologies.com/2019/09/20/micro-ammonia-cracker-foron-demand-mobile-hydrogen/ (accessed 2020-06-25).

(57) Tower Power Ltd. About Us http://towerpowerlimited.com/ about.php (accessed 2020-06-25).

(58) GenCell Ltd. GenCell Ltd. The Off-grid Challenge. https:// www.gencellenergy.com/our-products/gencell-a5/ (accessed 2020-0625).

(59) AFCEnergy. AFC Wraps Ammonia-to-Power Fuel Cell Generator Trials, Plans Commercial System. Fuel Cells Bull. 2019, 2019 (6), 11-12.

(60) STFC UK Researchers. Change Game for Hydrogen from Ammonia for Cars. Fuel Cells Bull. 2014, 2014 (7), 11.

(61) Fothergill, K.; Greenwood, S.; Makepeace, J.; Wilkinson, I. Ammonia to Green Hydrogen Project - Feasibility Study; Science and Technology Facilities Council, 2015; Vol. 33.

(62) Brown, Trevor. Engie, Siemens, STFC, and Ecuity awarded funding for green ammonia-to-hydrogen in UK. https://www. ammoniaenergy.org/articles/engie-siemens-stfc-and-ecuity-awardedfunding-for-green-ammonia-to-hydrogen-in-uk/ (accessed 2020-06$25)$.

(63) Powell, M. R.; Chellappa, A. S.; Vencill, T. R. Compact Fuel Cell Power Supplies with Safe Fuel Storage. Army Science Conference (24th) 2004, $1-10$.

(64) T-Raissi, A. Hydrogen from Ammonia and Ammonia-Borane Complex for Fuel Cell Applications. 2002 U.S. DOE Hydrogen Review 2002, 581-597.

(65) Rencat. Fuel Cell Grade $\mathrm{H}_{2}$ from ammonia without using Palladium membrane. https://www.rencat.net/Technology/ (accessed 2020-06-25).

(66) Badwal, S.; Giddey, S. S.; Kulkarni, F. T. C. A.; Hughes, A. E.; Kennedy, D. F. US Patent US 9,895,652 B2, 2018.

(67) ARPA-E. Funding for New Projects to Power Cleaner Transportation. Fuel Cells Bull. 2017, 2017 (1), 12-13.

(68) Perman, E. P.; Atkinson, G. A. S. The Decomposition of Ammonia by Heat. Proc. R. Soc. London 1905, 74, 110-117.

(69) Tyler, C. Ammonia as a Source of Hydrogen for Hardening Oils. J. Am. Oil Chem. Soc. 1934, 11, 231.

(70) Robertson, A. J. B.; Willhoft, E. M. A. Kinetics of the Decomposition of Ammonia on Platinum at Low Pressures. Trans. Faraday Soc. 1967, 63, 476-487.

(71) Willhoft, E. M. A. Kinetics of Decomposition of Ammonia at Low Pressures on Metal Surfaces. Trans. Faraday Soc. 1968, 64, 1925-1933. 
(72) Choudhary, T. V.; Santra, A. K.; Sivadinarayana, C.; Min, B. K.; Yi, C. W.; Davis, K.; Goodman, D. W. Ammonia Decomposition on $\operatorname{Ir}(100)$ : From Ultrahigh Vacuum to Elevated Pressures. Catal. Lett. 2001, 77 (1-3), 1-5.

(73) Sayas, S.; Morlanés, N.; Katikaneni, S. P.; Harale, A.; Solami, B.; Gascon, J. High Pressure Ammonia Decomposition on $\mathrm{Ru}-\mathrm{K} / \mathrm{CaO}$ Catalysts. Catal. Sci. Technol. 2020, 10 (15), 5027-5035.

(74) Carlo, A.; Di; Vecchione, L.; Del Prete, Z. Ammonia Decomposition over Commercial $\mathrm{Ru} / \mathrm{Al}_{2} \mathrm{O}_{3}$ Catalyst: An Experimental Evaluation at Different Operative Pressures and Temperatures. Int. J. Hydrogen Energy 2014, 39 (2), 808-814.

(75) Pitselis, G. E.; Petrolekas, P. D.; Vayenas, C. G. Electrochemical Promotion of Ammonia Decomposition over Fe Catalyst Films Interfaced with $\mathrm{K}^{+}$- \& $\mathrm{H}^{+}$- Conductors. Ionics 1997, 3 (1-2), 110-116.

(76) Marnellos, G.; Zisekas, S.; Stoukides, M. Synthesis of Ammonia at Atmospheric Pressure with the Use of Solid State Proton Conductors. J. Catal. 2000, 193 (1), 80-87.

(77) Zisekas, S.; Karagiannakis, G.; Marnellos, G.; Stoukides, M. Study of Ammonia Decomposition in a Proton Conducting Solid Electrolyte Cell. Ionics 2002, 8 (1-2), 118-122.

(78) Smith, C.; Essex, H. Effect of Electric Fields on the Decomposition of Ammonia by AlphaRays. J. Chem. Phys. 1938, 6 (4), 188-196.

(79) Zhao, Y.; Wang, L.; Zhang, J.; Gong, W.; Guo, H. Decomposition of Ammonia by Atmospheric Pressure AC Discharge: Catalytic Effect of the Electrodes. Catal. Today 2013, 211, 72-77.

(80) McLennan, J. C.; Greenwood, G. On the Decomposition of Ammonia by High-Speed Electrons. R. Soc. 1928, 120 (785), 283-295.

(81) Seabury, C. W.; Rhodin, T. N.; Purtell, R. J.; Merrill, R. P. Chemisorption and Reaction of $\mathrm{NH}_{3}$ on $\mathrm{Ni}(111)$. Surf. Sci. 1980, 93 (1), 117-126.

(82) Dawson, P. T.; Peng, Y. K. Ammonia Decomposition on Tungsten Surfaces. J. Chem. Phys. 1970, 52 (2), 1014-1015.

(83) Son, Y.-S.; Kim, K.-H.; Kim, K.-J.; Kim, J.-C. Ammonia Decomposition Using Electron Beam. Plasma Chem. Plasma Process. 2013, 33 (3), 617-629.

(84) Hirabayashi, S.; Ichihashi, M. Adsorption and Dehydrogenation of Ammonia on Vanadium and Niobium Nitride Cluster Cations. Int. J. Mass Spectrom. 2016, 407, 86-91.

(85) Guler, M.; Dogu, T.; Varışl1, D. Hydrogen Production over Molybdenum Loaded Mesoporous Carbon Catalysts in Microwave Heated Reactor System. Appl. Catal., B 2017, 219, 173-182.

(86) Varışlı, D.; Korkusuz, C.; Dogu, T. Microwave-Assisted Ammonia Decomposition Reaction over Iron Incorporated Mesoporous Carbon Catalysts. Appl. Catal., B 2017, 201, 370-380.

(87) Barker, R. Decomposition of Ammonia in a Microwave Discharge. J. Chem. Soc., Faraday Trans. 1 1972, 68, 315-322.

(88) Carbaugh, D. C.; Munno, F. J.; Marchello, J. M. Ammonia Decomposition in Glow Discharge. J. Chem. Phys. 1967, 47 (12), 5211-5214.

(89) Wang, L.; Zhao, Y.; Liu, C.; Gong, W.; Guo, H. Plasma Driven Ammonia Decomposition on a Fe-Catalyst: Eliminating Surface Nitrogen Poisoning. Chem. Commun. 2013, 49 (36), 3787-3789.

(90) Yi, Y.; Wang, L.; Guo, Y.; Sun, S.; Guo, H. Plasma-Assisted Ammonia Decomposition over $\mathrm{Fe}-\mathrm{Ni}$ Alloy Catalysts for $\mathrm{CO}_{\mathrm{x}}$-Free Hydrogen. AIChE J. 2018, 65 (2), 691-701.

(91) Hayakawa, Y.; Miura, T.; Shizuya, K.; Wakazono, S.; Tokunaga, K.; Kambara, S. Hydrogen Production System Combined with a Catalytic Reactor and a Plasma Membrane Reactor from Ammonia. Int. J. Hydrogen Energy 2019, 44 (20), 9987-9993.

(92) Inoue, Y.; Hayakawa, Y.; Takeyama, A.; Miura, T.; Kambara, S. Hydrogen Production by Ammonia Decomposition Using Pulsed Plasma. International Symposium on Electrohydrodynamics (ISEHD) 2014, 2-5.

(93) Goto, Y.; Hayakawa, Y.; Kambara, S. Reaction Mechanism of Ammonia Decomposition by Atmospheric Plasma. Ninth JSME-KSME Thermal and Fluids Engineering Conference 2017, 1-4.

(94) Akiyama, M.; Aihara, K.; Sawaguchi, T.; Matsukata, M.; Iwamoto, M. Ammonia Decomposition to Clean Hydrogen Using
Non-Thermal Atmospheric-Pressure Plasma. Int. J. Hydrogen Energy 2018, 43 (31), 14493-14497.

(95) Wang, L.; Yi, Y.; Zhao, Y.; Zhang, R.; Zhang, J.; Guo, H. $\mathrm{NH}_{3}$ Decomposition for $\mathrm{H}_{2}$ Generation: Effects of Cheap Metals and Supports on Plasma-Catalyst Synergy. ACS Catal. 2015, 5 (7), 41674174.

(96) Hu, T.; Wang, Y. Effect of Operating and Geometrical Parameters on Ammonia Decomposition in a Tubular Reactor Driven by Concentrating Solar Power. J. Energy Eng. 2020, 146 (4), 040200181-040200188.

(97) Wang, B.; Kong, H.; Wang, H.; Wang, Y.; Hu, X. Kinetic and Thermodynamic Analyses of Mid/Low-Temperature Ammonia Decomposition in Solar-Driven Hydrogen Permeation Membrane Reactor. Int. J. Hydrogen Energy 2019, 44 (49), 26874-26887.

(98) Chen, J.; Yan, L. Ammonia Decomposition Coupled with Methane Combustion in Catalytic Microreactors for Hydrogen Production. Chem. Biomol. Eng. 2017, 2 (1), 19-26.

(99) Engelbrecht, N.; Chiuta, S.; Bessarabov, D. G. A Highly Efficient Autothermal Microchannel Reactor for Ammonia Decomposition: Analysis of Hydrogen Production in Transient and Steady-State Regimes. J. Power Sources 2018, 386, 47-55.

(100) Chiuta, S.; Bessarabov, D. G. Design and Operation of an Ammonia-Fueled Microchannel Reactor for Autothermal Hydrogen Production. Catal. Today 2018, 310 (1), 187-194.

(101) Kim, J. H.; Um, D. H.; Kwon, O. C. Hydrogen Production from Burning and Reforming of Ammonia in a Microreforming System. Energy Convers. Manage. 2012, 56, 184-191.

(102) Kim, J. H.; Kwon, O. C. A Micro Reforming System Integrated with a Heat-Recirculating Micro-Combustor to Produce Hydrogen from Ammonia. Int. J. Hydrogen Energy 2011, 36 (3), 1974-1983.

(103) Arana, L. R.; Schaevitz, S. B.; Franz, A. J.; Schmidt, M. A.; Jensen, K. F. A Microfabricated Suspended-Tube Chemical Reactor for Thermally Efficient Fuel Processing. J. Microelectromech. Syst. 2003, 12 (5), 600-612.

(104) Kaisare, N. S.; Stefanidis, G. D.; Vlachos, D. G. Millisecond Production of Hydrogen from Alternative, High Hydrogen Density Fuels in a Cocurrent Multifunctional Microreactor. Ind. Eng. Chem. Res. 2009, 48 (4), 1749-1760.

(105) Regatte, V. R.; Kaisare, N. S. Hydrogen Generation in Spatially Coupled Cross-Flow Microreactors. Chem. Eng. J. 2013, 215-216, $876-885$.

(106) Deshmukh, S. R.; Vlachos, D. G. Effect of Flow Configuration on the Operation of Coupled Combustor/Reformer Microdevices for Hydrogen Production. Chem. Eng. Sci. 2005, 60 (21), 5718-5728.

(107) Hanada, N.; Hino, S.; Ichikawa, T.; Suzuki, H.; Takai, K.; Kojima, Y. Hydrogen Generation by Electrolysis of Liquid Ammonia. Chem. Commun. 2010, 46 (41), 7775-7777.

(108) Modisha, P.; Bessarabov, D. Electrocatalytic Process for Ammonia Electrolysis: A Remediation Technique with Hydrogen Co-Generation. Int. J. Electrochem. Sci. 2016, 11 (8), 6627-6635.

(109) Reli, M.; Ambrožová, N.; Šihor, M.; Matějová, L.; Čapek, L.; Obalová, L.; Matěj, Z.; Kotarba, A.; Kočí, K. Novel Cerium Doped Titania Catalysts for Photocatalytic Decomposition of Ammonia. Appl. Catal., B 2015, 178, 108-116.

(110) Iwase, A.; Ii, K.; Kudo, A. Decomposition of an Aqueous Ammonia Solution as a Photon Energy Conversion Reaction Using a Ru-Loaded ZnS Photocatalyst. Chem. Commun. 2018, 54 (48), 61176119.

(111) Wiig, E. O.; Kistiakowsky, G. B. The Photochemical Decomposition of Ammonia. J. Am. Chem. Soc. 1932, 54 (5), 18061820.

(112) Li, Q.-s.; Domen, K.; Naito, S.; Onishi, T.; Tamaru, K. Photocatalytic Synthesis and Photodecomposition of Ammonia over $\mathrm{SrTiO}_{3}$ and $\mathrm{BaTiO}_{3}$ Based Catalysts. Chem. Lett. 1983, 12, 321-324.

(113) Yuzawa, H.; Mori, T.; Itoh, H.; Yoshida, H. Reaction Mechanism of Ammonia Decomposition to Nitrogen and Hydrogen over Metal Loaded Titanium Oxide Photocatalyst. J. Phys. Chem. C 2012, 116 (6), 4126-4136. 
(114) Paik, B.; Tsubota, M.; Ichikawa, T.; Kojima, Y. Catalytic Effect of $\mathrm{ATiO}_{3}(\mathrm{~A}=\mathrm{Sr}, \mathrm{Ba})$ on Ammonia Decomposition during Mechanical Milling. Chem. Commun. 2010, 46 (22), 3982-3984.

(115) Li, L.; Hurley, J. A. Ammonia-Based Hydrogen Source for Fuel Cell Applications. Int. J. Hydrogen Energy 2007, 32 (1), 6-10.

(116) White, A. H.; Melville, W. The Decomposition of Ammonia at High Temperatures. J. Am. Chem. Soc. 1905, 27 (4), 373-386.

(117) Chambers, A.; Yoshii, Y.; Inada, T.; Miyamoto, T. Ammonia Decomposition in Coal Gasification Atmospheres. Can. J. Chem. Eng. 1996, 74 (6), 929-934.

(118) Wang, W.; Padban, N.; Ye, Z.; Andersson, A.; Bjerle, I. Kinetics of Ammonia Decomposition in Hot Gas Cleaning. Ind. Eng. Chem. Res. 1999, 38 (11), 4175-4182.

(119) Mojtahedi, W.; Abbasian, J. Catalytic Decomposition of Ammonia in a Fuel Gas at High Temperature and Pressure. Fuel 1995, 74 (11), 1698-1703.

(120) Simell, P. A.; Hepola, J. O.; Krause, A. O. I. Effects of Gasification Gas Components on Tar and Ammonia Decomposition over Hot Gas Cleanup Catalysts. Fuel 1997, 76 (12), 1117-1127.

(121) Platonov, O. I.; Stepanov, E. N. Monitoring Catalyst Activity in Removing Ammonia from Coke-Oven Gas. Coke Chem. 2008, 51 (4), 153-154.

(122) Pansare, S. S.; Goodwin, J. G., Jr. Ammonia Decomposition on Tungsten-Based Catalysts in the Absence and Presence of Syngas. Ind. Eng. Chem. Res. 2008, 47 (12), 4063-4070.

(123) Ohtsuka, Y.; Xu, C.; Kong, D.; Tsubouchi, N. Decomposition of Ammonia with Iron and Calcium Catalysts Supported on Coal Chars. Fuel 2004, 83 (6), 685-692.

(124) Collins, J. P.; Way, J. D.; Kraisuwansarn, N. A Mathematical Model of a Catalytic Membrane Reactor for the Decomposition of $\mathrm{NH}_{3}$. J. Membr. Sci. 1993, 77 (2-3), 265-282.

(125) Gobina, E. N.; Oklany, J. S.; Hughes, R. Elimination of Ammonia from Coal Gasification Streams by Using a Catalytic Membrane Reactor. Ind. Eng. Chem. Res. 1995, 34 (11), 3777-3783.

(126) Abashar, M. E. E. Integrated Catalytic Membrane Reactors for Decomposition of Ammonia. Chem. Eng. Process. 2002, 41 (5), 403412.

(127) Abashar, M. E. E.; Al-Sughair, Y. S.; Al-Mutaz, I. S. Investigation of Low Temperature Decomposition of Ammonia Using Spatially Patterned Catalytic Membrane Reactors. Appl. Catal., A 2002, 236 (12), 35-53.

(128) Tsubouchi, N.; Hashimoto, H.; Ohtsuka, Y. Sulfur Tolerance of an Inexpensive Limonite Catalyst for High Temperature Decomposition of Ammonia. Powder Technol. 2008, 180 (1-2), 184-189.

(129) Uemiya, S.; Uchida, M.; Moritomi, H.; Yoshiie, R.; Nishimura, M. Ammonia Decomposition Catalyst with Resistance to Coexisting Sulfur Compounds. Mater. Trans. 2005, 46 (12), 2709-2712.

(130) Wang, W.; Padban, N.; Ye, Z.; Olofsson, G.; Andersson, A.; Bjerle, I. Catalytic Hot Gas Cleaning of Fuel Gas from an Air-Blown Pressurized Fluidized-Bed Gasifier. Ind. Eng. Chem. Res. 2000, 39 (11), 4075-4081.

(131) Arabczyk, W.; Narkiewicz, U. A New Method for in Situ Determination of Number of Active Sites in Iron Catalysts for Ammonia Synthesis and Decomposition. Appl. Surf. Sci. 2002, 196 (14), 423-428.

(132) He, C.; Wang, H.; Huai, L.; Liu, J. Mechanism of Ammonia Decomposition and Oxidation on $\operatorname{Ir}(100)$ : A First-Principles Study. J. Phys. Chem. C 2012, 116 (45), 24035-24045.

(133) Hung, C.-M. Catalytic Decomposition of Ammonia over Bimetallic $\mathrm{CuO} / \mathrm{CeO}_{2}$ Nanoparticle Catalyst. Aerosol Air Qual. Res. 2008, 8 (4), 447-458.

(134) Lee, J. Y.; Lim, Y. H.; Park, B. H.; Adelodun, A. A.; Jo, Y. M. Preparation of $\mathrm{Ag}-\mathrm{Cu} / \mathrm{Al}_{2} \mathrm{O}_{3}$ Composite Catalyst for Ammonia Decomposition. Bull. Korean Chem. Soc. 2015, 36 (1), 162-167.

(135) Bera, P.; Hegde, M. S. Oxidation and Decomposition of $\mathrm{NH}_{3}$ over Combustion Synthesized $\mathrm{Al}_{2} \mathrm{O}_{3}$ and $\mathrm{CeO}_{2}$ Supported $\mathrm{Pt}$, $\mathrm{Pd}$ and Ag Catalysts. Indian J. Chem. - Sect. A Inorganic, Phys. Theor. Anal. Chem. 2002, 41 (8), 1554-1561.
(136) Nagaoka, K.; Eboshi, T.; Takeishi, Y.; Tasaki, R.; Honda, K.; Imamura, K.; Sato, K. Carbon-Free $\mathrm{H}_{2}$ Production from Ammonia Triggered at Room Temperature with an Acidic $\mathrm{RuO}_{2} / \gamma-\mathrm{Al}_{2} \mathrm{O}_{3}$ Catalyst. Sci. Adv. 2017, 3 (4), 1-8.

(137) Goto, M.; Shiramizu, D.; Kodama, A.; Hirose, T. Kinetic Analysis for Ammonia Decomposition in Supercritical Water Oxidation of Sewage Sludge. Ind. Eng. Chem. Res. 1999, 38 (11), 4500-4503.

(138) Friedlander, A. G.; Courty, P. R.; Montarnal, R. E. Ammonia Decomposition in the Presence of Water Vapor. II. Kinetics of the Reaction on Nickel Catalyst. J. Catal. 1977, 48 (1-3), 322-332.

(139) Atsumi, R.; Noda, R.; Takagi, H.; Vecchione, L.; Di Carlo, A.; Del Prete, Z.; Kuramoto, K. Effects of Steam on $\mathrm{Ni} / \mathrm{Al}_{2} \mathrm{O}_{3}$ Catalysts for Ammonia Decomposition. Ind. Eng. Chem. Res. 2014, 53 (45), 1784917853.

(140) Hung, C.-M. Decomposition Kinetics of Ammonia in Gaseous Stream by a Nanoscale Copper-Cerium Bimetallic Catalyst. J. Hazard. Mater. 2008, 150 (1), 53-61.

(141) Makepeace, J. W.; He, T.; Weidenthaler, C.; Jensen, T. R.; Chang, F.; Vegge, T.; Ngene, P.; Kojima, Y.; de Jongh, P. E.; Chen, P.; David, W. I. F. Reversible Ammonia-Based and Liquid Organic Hydrogen Carriers for High-Density Hydrogen Storage: Recent Progress. Int. J. Hydrogen Energy 2019, 44 (15), 7746-7767.

(142) Dixon, J. K. The Kinetics of the Decomposition of Ammonia on Copper. J. Am. Chem. Soc. 1931, 53 (5), 1763-1773.

(143) Mukherjee, S.; Devaguptapu, S. V.; Sviripa, A.; Lund, C. R. F.; Wu, G. Low-Temperature Ammonia Decomposition Catalysts for Hydrogen Generation. Appl. Catal., B 2018, 226, 162-181.

(144) Bell, T. E.; Torrente-Murciano, L. $\mathrm{H}_{2}$ Production via Ammonia Decomposition Using Non-Noble Metal Catalysts: A Review. Top. Catal. 2016, 59 (15-16), 1438-1457.

(145) Schüth, F.; Palkovits, R.; Schlögl, R.; Su, D. S. Ammonia as a Possible Element in an Energy Infrastructure: Catalysts for Ammonia Decomposition. Energy Environ. Sci. 2012, 5 (4), 6278-6289.

(146) Chemical elements by market price. 13 Nov. 2020, http://www. leonland.de/elements by price/en/list (accessed 2020-11-13).

(147) Nuss, P.; Eckelman, M. J. Life Cycle Assessment of Metals: A Scientific Synthesis. PLoS One 2014, 9 (7), e101298.

(148) García-Bordejé, E.; Armenise, S.; Roldán, L. Toward Practical Application of $\mathrm{H}_{2}$ Generation from Ammonia Decomposition Guided by Rational Catalyst Design. Catal. Rev.: Sci. Eng. 2014, 56 (2), 220237.

(149) Cheddie, D. Ammonia as a Hydrogen Source for Fuel Cells: A Review. Hydrogen Energy - Challenges and Perspectives 2012, 333-362.

(150) Abd Ali, Z. D.; Allami, S.; Jwad, B. H. Ammonia as Hydrogen Storage Media, Sustainable Method to Hydrogen Evolution. J. Phys.: Conf. Ser. 2018, 1032 (1), 1-10.

(151) Yin, S. F.; Xu, B. Q.; Zhou, X. P.; Au, C. T. A Mini-Review on Ammonia Decomposition Catalysts for on-Site Generation of Hydrogen for Fuel Cell Applications. Appl. Catal., A 2004, 277 (1-2), 1-9.

(152) Duan, X.; Zhou, X.; Chen, D. Structural Manipulation of the Catalysts for Ammonia Decomposition. Catalysis 2013, 25, 118-140.

(153) Boisen, A.; Dahl, S.; Nørskov, J. K.; Christensen, C. H. Why the Optimal Ammonia Synthesis Catalyst Is Not the Optimal Ammonia Decomposition Catalyst. J. Catal. 2005, 230 (2), 309-312.

(154) Jacobsen, C. J. H.; Dahl, S.; Boisen, A.; Clausen, B. S.; Topsøe, H.; Logadottir, A.; Nørskov, J. K. Optimal Catalyst Curves: Connecting Density Functional Theory Calculations with Industrial Reactor Design and Catalyst Selection. J. Catal. 2002, 205 (2), 382-387.

(155) Ganley, J. C.; Thomas, F. S.; Seebauer, E. G.; Masel, R. I. A Priori Catalytic Activity Correlations: The Difficult Case of Hydrogen Production from Ammonia. Catal. Lett. 2004, 96 (3-4), 117-122.

(156) Yin, S.-F.; Zhang, Q.-H.; Xu, B.-Q.; Zhu, W.-X.; Ng, C.-F.; Au, C.-T. Investigation on the Catalysis of $\mathrm{CO}_{\mathrm{x}}$-Free Hydrogen Generation from Ammonia. J. Catal. 2004, 224 (2), 384-396.

(157) Liu, H.; Wang, H.; Shen, J.; Ying, S.; Liu, Z. Preparation and Evaluation of Ammonia Decomposition Catalysts by High-Throughput Technique. React. Kinet. Catal. Lett. 2008, 93 (1), 11-17. 
(158) Choudhary, T. V.; Sivadinarayana, C.; Goodman, D. W. Catalytic Ammonia Decomposition : $\mathrm{CO}_{\mathrm{x}}$-Free Hydrogen Production for Fuel Cell Applications. Catal. Lett. 2001, 72 (3-4), 197-201.

(159) Choudhary, T. V.; Goodman, D. W. CO-Free Fuel Processing for Fuel Cell Applications. Catal. Today 2002, 77, 65-78.

(160) Li, Y.; Liu, S.; Yao, L.; Ji, W.; Au, C.-T. Core-Shell Structured Iron Nanoparticles for the Generation of $\mathrm{CO}_{\mathrm{x}}$-Free Hydrogen via Ammonia Decomposition. Catal. Commun. 2010, 11 (5), 368-372.

(161) Yao, L.; Shi, T.; Li, Y.; Zhao, J.; Ji, W.; Au, C.-T. Core-Shell Structured Nickel and Ruthenium Nanoparticles: Very Active and Stable Catalysts for the Generation of $\mathrm{CO}_{\mathrm{x}}$-Free Hydrogen via Ammonia Decomposition. Catal. Today 2011, 164 (1), 112-118.

(162) Yao, L. H.; Li, Y. X.; Zhao, J.; Ji, W. J.; Au, C. T. Core-Shell Structured Nanoparticles ( $\left.\mathrm{M} @ \mathrm{SiO}_{2}, \mathrm{Al}_{2} \mathrm{O}_{3}, \mathrm{MgO} ; \mathrm{M}=\mathrm{Fe}, \mathrm{Co}, \mathrm{Ni}, \mathrm{Ru}\right)$ and Their Application in $\mathrm{CO}_{\mathrm{x}}$-Free $\mathrm{H}_{2}$ Production via $\mathrm{NH}_{3}$ Decomposition. Catal. Today 2010, 158 (3-4), 401-408.

(163) Zhang, J.; Comotti, M.; Schüth, F.; Schlögl, R.; Su, D. S. Commercial Fe- or Co-Containing Carbon Nanotubes as Catalysts for $\mathrm{NH}_{3}$ Decomposition. Chem. Commun. 2007, 19, 1916-1918.

(164) Li, X.-K.; Ji, W.-J.; Zhao, J.; Wang, S.-J.; Au, C.-T. Ammonia Decomposition over $\mathrm{Ru}$ and $\mathrm{Ni}$ Catalysts Supported on Fumed $\mathrm{SiO}_{2}$, MCM-41, and SBA-15. J. Catal. 2005, 236 (2), 181-189.

(165) Seehra, M. S.; Bristow, A. D. Introductory Chapter: Overview of the Properties and Applications of Noble and Precious Metals. Noble and Precious Metals - Properties, Nanoscale Effects and Applications 2018, $3-11$.

(166) Papapolymerou, G.; Bontozoglou, V. Decomposition of $\mathrm{NH}_{3}$, on Pd and Ir Comparison with Pt and Rh. J. Mol. Catal. A: Chem. 1997, 120, 165-171.

(167) Hill, A. K.; Torrente-Murciano, L. In-Situ $\mathrm{H}_{2}$ Production via Low Temperature Decomposition of Ammonia: Insights into the Role of Cesium as a Promoter. Int. J. Hydrogen Energy 2014, 39 (15), 76467654.

(168) Hill, A. K.; Torrente-Murciano, L. Low Temperature $\mathrm{H}_{2}$ Production from Ammonia Using Ruthenium-Based Catalysts: Synergetic Effect of Promoter and Support. Appl. Catal., B 2015, 172-173, 129-135.

(169) Wang, S. J.; Yin, S. F.; Li, L.; Xu, B. Q.; Ng, C. F.; Au, C. T. Investigation on Modification of Ru/CNTs Catalyst for the Generation of $\mathrm{CO}_{\mathrm{x}}$-Free Hydrogen from Ammonia. Appl. Catal., B 2004, 52 (4), 287-299.

(170) Raróg-Pilecka, W.; Kowalczyk, Z.; Sentek, J.; Składanowski, D.; Szmigiel, D.; Zieliñski, J. Decomposition of Ammonia over Potassium Promoted Ruthenium Catalyst Supported on Carbon. Appl. Catal., A 2001, 208 (1-2), 213-216.

(171) Ren, S.; Huang, F.; Zheng, J.; Chen, S.; Zhang, H. Ruthenium Supported on Nitrogen-Doped Ordered Mesoporous Carbon as Highly Active Catalyst for $\mathrm{NH}_{3}$ Decomposition to $\mathrm{H}_{2}$. Int. J. Hydrogen Energy 2017, 42 (8), 5105-5113.

(172) Bell, T. E.; Zhan, G.; Wu, K.; Zeng, H. C.; Torrente-Murciano, L. Modification of Ammonia Decomposition Activity of Ruthenium Nanoparticles by N-Doping of CNT Supports. Top. Catal. 2017, 60 (15-16), 1251-1259.

(173) García-García, F. R.; Álvarez-Rodríguez, J.; Rodríguez-Ramos, I.; Guerrero-Ruiz, A. The Use of Carbon Nanotubes with and without Nitrogen Doping as Support for Ruthenium Catalysts in the Ammonia Decomposition Reaction. Carbon 2010, 48 (1), 267-276.

(174) Chen, J.; Zhu, Z. H.; Wang, S.; Ma, Q.; Rudolph, V.; Lu, G. Q. Effects of Nitrogen Doping on the Structure of Carbon Nanotubes (CNTs) and Activity of Ru/CNTs in Ammonia Decomposition. Chem. Eng. J. 2010, 156 (2), 404-410.

(175) Marco, Y.; Roldán, L.; Armenise, S.; García-Bordejé, E. Support-Induced Oxidation State of Catalytic Ru Nanoparticles on Carbon Nanofibers That Were Doped with Heteroatoms (O, N) for the Decomposition of $\mathrm{NH}_{3}$. ChemCatChem 2013, 5 (12), 3829-3834.

(176) Duan, X.; Zhou, J.; Qian, G.; Li, P.; Zhou, X.; Chen, D. Carbon Nanofiber-Supported $\mathrm{Ru}$ Catalysts for Hydrogen Evolution by Ammonia Decomposition. Chin. J. Catal. 2010, 31 (8), 979-986.
(177) García-García, F. R.; Guerrero-Ruiz, A.; Rodríguez-Ramos, I. Role of B5-Type Sites in Ru Catalysts Used for the $\mathrm{NH}_{3}$ Decomposition Reaction. Top. Catal. 2009, 52 (6-7), 758-764.

(178) García-García, F. R.; Gallegos-Suarez, E.; Fernández-García, M.; Guerrero-Ruiz, A.; Rodríguez-Ramos, I. Understanding the Role of Oxygen Surface Groups: The Key for a Smart Ruthenium-Based Carbon-Supported Heterogeneous Catalyst Design and Synthesis. Appl. Catal., A 2017, 544, 66-76.

(179) Yin, S.-F.; Xu, B.-Q.; Ng, C.-F.; Au, C.-T. Nano Ru/CNTs: A Highly Active and Stable Catalyst for the Generation of $\mathrm{CO}_{\mathrm{x}}$-Free Hydrogen in Ammonia Decomposition. Appl. Catal., B 2004, 48 (4), 237-241.

(180) Yin, S. F.; Xu, B. Q.; Zhu, W. X.; Ng, C. F.; Zhou, X. P.; Au, C. T. Carbon Nanotubes-Supported $\mathrm{Ru}$ Catalyst for the Generation of $\mathrm{CO}_{\mathrm{x}}-$ Free Hydrogen from Ammonia. Catal. Today 2004, 93-95, 27-38.

(181) Petrunin, D. A.; Borisov, V. A.; Iost, K. N.; Temerev, V. L.; Trenikhin, M. V.; Gulyaeva, T. I.; Shlyapin, D. A.; Tsyrulnikov, P. G. Comparison of the Activity of $\mathrm{Ru}-\mathrm{K} /$ Sibunit Catalysts in Ammonia Synthesis and Decomposition. AIP Conf. Proc. 2018, 2141, 02002410200247.

(182) Li, G.; Kanezashi, M.; Tsuru, T. Catalytic Ammonia Decomposition over High-Performance $\mathrm{Ru}$ /Graphene Nanocomposites for Efficient $\mathrm{CO}_{\mathrm{x}}$-Free Hydrogen Production. Catalysts 2017, 7 (23), $1-12$.

(183) Li, L.; Zhu, Z. H.; Yan, Z. F.; Lu, G. Q.; Rintoul, L. Catalytic Ammonia Decomposition over Ru/Carbon Catalysts: The Importance of the Structure of Carbon Support. Appl. Catal., A 2007, 320, 166172.

(184) Li, L.; Zhu, Z. H.; Wang, S. B.; Yao, X. D.; Yan, Z. F. Chromium Oxide Catalysts for $\mathrm{CO}_{\mathrm{x}}$-Free Hydrogen Generation via Catalytic Ammonia Decomposition. J. Mol. Catal. A: Chem. 2009, 304 (1-2), $71-76$.

(185) Raróg-Pilecka, W.; Szmigiel, D.; Kowalczyk, Z.; Jodzis, S.; Zieliñski, J. Ammonia Decomposition over the Carbon-Based Ruthenium Catalyst Promoted with Barium or Cesium. J. Catal. 2003, 218 (2), 465-469.

(186) Huang, D.-C.; Jiang, C.-H.; Liu, F.-J.; Cheng, Y.-C.; Chen, Y.C.; Hsueh, K.-L. Preparation of Ru-Cs Catalyst and Its Application on Hydrogen Production by Ammonia Decomposition. Int. J. Hydrogen Energy 2013, 38 (8), 3233-3240.

(187) Li, L.; Zhu, Z. H.; Lu, G. Q.; Yan, Z. F.; Qiao, S. Z. Catalytic Ammonia Decomposition over CMK-3 Supported Ru Catalysts: Effects of Surface Treatments of Supports. Carbon 2007, 45 (1), 1120.

(188) Yin, S. F.; Xu, B. Q.; Wang, S. J.; Ng, C. F.; Au, C. T. MagnesiaCarbon Nanotubes (MgO-CNTs) Nanocomposite: Novel Support of $\mathrm{Ru}$ Catalyst for the Generation of $\mathrm{CO}_{\mathrm{x}}$-Free Hydrogen from Ammonia. Catal. Lett. 2004, 96 (3-4), 113-116.

(189) Bajus, S.; Agel, F.; Kusche, M.; Ní Bhriain, N.; Wasserscheid, P. Alkali Hydroxide-Modified $\mathrm{Ru} / \gamma-\mathrm{Al}_{2} \mathrm{O}_{3}$ Catalysts for Ammonia Decomposition. Appl. Catal., A 2016, 510, 189-195.

(190) Pyrz, W.; Vijay, R.; Binz, J.; Lauterbach, J.; Buttrey, D. J. Characterization of K-Promoted Ru Catalysts for Ammonia Decomposition Discovered Using High-Throughput Experimentation. Top. Catal. 2008, 50 (1-4), 180-191.

(191) Klerke, A.; Klitgaard, S. K.; Fehrmann, R. Catalytic Ammonia Decomposition Over Ruthenium Nanoparticles Supported on NanoTitanates. Catal. Lett. 2009, 130 (3-4), 541-546.

(192) Chung, D. B.; Kim, H. Y.; Jeon, M.; Lee, D. H.; Park, H. S.; Choi, S. H.; Nam, S. W.; Jang, S. C.; Park, J.-H.; Lee, K.-Y.; Yoon, C. W. Enhanced Ammonia Dehydrogenation over $\mathrm{Ru} / \mathrm{La}(\mathrm{x})-\mathrm{Al}_{2} \mathrm{O}_{3}(\mathrm{X}=0-$ $50 \mathrm{Mol} \%)$ : Structural and Electronic Effects of La Doping. Int. J. Hydrogen Energy 2017, 42 (3), 1639-1647.

(193) Huang, C.; Yu, Y.; Yang, J.; Yan, Y.; Wang, D.; Hu, F.; Wang, X.; Zhang, R.; Feng, G. $\mathrm{Ru} / \mathrm{La}_{2} \mathrm{O}_{3}$ Catalyst for Ammonia Decomposition to Hydrogen. Appl. Surf. Sci. 2019, 476, 928-936.

(194) Szmigiel, D.; Raróg-Pilecka, W.; Miśkiewicz, E.; Kaszkur, Z.; Kowalczyk, Z. Ammonia Decomposition over the Ruthenium Catalysts 
Deposited on Magnesium-Aluminum Spinel. Appl. Catal., A 2004, 264 (1), 59-63.

(195) Su, Q.; Gu, L. L.; Zhong, A. H.; Yao, Y.; Ji, W.J.; Ding, W. P.; Au, C.-T. Layered Double Hydroxide Derived $\mathrm{Mg}_{2} \mathrm{Al}-\mathrm{LDO}$ Supported and K-Modified $\mathrm{Ru}$ Catalyst for Hydrogen Production via Ammonia Decomposition. Catal. Lett. 2018, 149 (9), 1-10.

(196) Tan, H.; Li, K.; Sioud, S.; Cha, D.; Amad, M. H.; Hedhili, M. N.; Al-Talla, Z. A. Synthesis of Ru Nanoparticles Confined in Magnesium Oxide-Modified Mesoporous Alumina and Their Enhanced Catalytic Performance during Ammonia Decomposition. Catal. Commun. 2012, 26, 248-252.

(197) Wang, Z.; Qu, Y.; Shen, X.; Cai, Z. Ruthenium Catalyst Supported on Ba Modified $\mathrm{ZrO}_{2}$ for Ammonia Decomposition to $\mathrm{CO}_{\mathrm{x}}$ Free Hydrogen. Int. J. Hydrogen Energy 2019, 44 (14), 7300-7307.

(198) Lorenzut, B.; Montini, T.; Pavel, C. C.; Comotti, M.; Vizza, F.; Bianchini, C.; Fornasiero, P. Embedded $\mathrm{Ru} @ \mathrm{ZrO}_{2}$ Catalysts for $\mathrm{H}_{2}$ Production by Ammonia Decomposition. ChemCatChem 2010, 2 (9), 1096-1106.

(199) Miyamoto, M.; Hamajima, A.; Oumi, Y.; Uemiya, S. Effect of Basicity of Metal Doped $\mathrm{ZrO}_{2}$ Supports on Hydrogen Production Reactions. Int. J. Hydrogen Energy 2018, 43 (2), 730-738.

(200) Yin, S.-F.; Xu, B.-Q.; Wang, S.-J.; Au, C.-T. Nanosized Ru on High-Surface-Area Superbasic $\mathrm{ZrO}_{2}-\mathrm{KOH}$ for Efficient Generation of Hydrogen via Ammonia Decomposition. Appl. Catal., A 2006, 301 (2), 202-210.

(201) Zhang, J.; Xu, H.; Ge, Q.; Li, W. Highly Efficient Ru/MgO Catalysts for $\mathrm{NH}_{3}$ Decomposition: Synthesis, Characterization and Promoter Effect. Catal. Commun. 2006, 7 (3), 148-152.

(202) Ju, X.; Liu, L.; Yu, P.; Guo, J.; Zhang, X.; He, T.; Wu, G.; Chen, P. Mesoporous $\mathrm{Ru} / \mathrm{MgO}$ Prepared by a Deposition-Precipitation Method as Highly Active Catalyst for Producing $\mathrm{CO}_{\mathrm{x}}$-Free Hydrogen from Ammonia Decomposition. Appl. Catal., B 2017, 211, 167-175.

(203) Li, J.; Wang, W.; Chen, W.; Gong, Q.; Luo, J.; Lin, R.; Xin, H.; Zhang, H.; Wang, D.; Peng, Q.; Zhu, W.; Chen, C.; Li, Y. Sub-Nm Ruthenium Cluster as an Efficient and Robust Catalyst for Decomposition and Synthesis of Ammonia: Break the "Size Shackles. Nano Res. 2018, 11 (9), 4774-4785.

(204) Kurtoğlu, S. F.; Soyer-Uzun, S.; Uzun, A. Utilizing Red Mud Modified by Simple Treatments as a Support to Disperse Ruthenium Provides a High and Stable Performance for $\mathrm{CO}_{\mathrm{x}}$-Free Hydrogen Production from Ammonia. Catal. Today 2020, 357, 1-11.

(205) Ng, P. F.; Li, L.; Wang, S.; Zhu, Z.; Lu, G.; Yan, Z. Catalytic Ammonia Decomposition over Industrial-Waste-Supported Ru Catalysts. Environ. Sci. Technol. 2007, 41 (10), 3758-3762.

(206) Li, L.; Wang, S.; Zhu, Z.; Yao, X.; Yan, Z. Catalytic Decomposition of Ammonia over Fly Ash Supported Ru Catalysts. Fuel Process. Technol. 2008, 89 (11), 1106-1112.

(207) Varışl1, D.; Elverisli, E. E. Synthesizing Hydrogen from Ammonia over $\mathrm{Ru}$ Incorporated $\mathrm{SiO}_{2}$ Type Nanocomposite Catalysts. Int. J. Hydrogen Energy 2014, 39 (20), 10399-10408.

(208) Li, Y.; Yao, L.; Song, Y.; Liu, S.; Zhao, J.; Ji, W.; Au, C.-T. CoreShell Structured Microcapsular-like $\mathrm{Ru} @ \mathrm{SiO}_{2}$ Reactor for Efficient Generation of $\mathrm{CO}_{\mathrm{x}}$-Free Hydrogen through Ammonia Decomposition. Chem. Commun. 2010, 46 (29), 5298-5300.

(209) Wang, L.; Chen, J.; Ge, L.; Zhu, Z.; Rudolph, V. HalloysiteNanotube-Supported $\mathrm{Ru}$ Nanoparticles for Ammonia Catalytic Decomposition to Produce $\mathrm{CO}_{\mathrm{x}}$-Free Hydrogen. Energy Fuels 2011, 25 (8), 3408-3416.

(210) Hu, X.-C.; Fu, X.-P.; Wang, W.-W.; Wang, X.; Wu, K.; Si, R.; Ma, C.; Jia, C.-J.; Yan, C.-H. Ceria-Supported Ruthenium Clusters Transforming from Isolated Single Atoms for Hydrogen Production via Decomposition of Ammonia. Appl. Catal., B 2020, 268, 118424.

(211) Hu, Z.; Mahin, J.; Datta, S.; Bell, T. E.; Torrente-Murciano, L. $\mathrm{Ru}$-Based Catalysts for $\mathrm{H}_{2}$ Production from Ammonia: Effect of $1 \mathrm{D}$ Support. Top. Catal. 2019, 62 (17-20), 1169-1177.

(212) Zhao, J.; Xu, S.; Wu, H.; You, Z.; Deng, L.; Qiu, X. MetalSupport Interactions on $\mathrm{Ru} / \mathrm{CaAlO} \mathrm{X}_{\mathrm{X}}$ Catalysts Derived from Structural Reconstruction of Ca-Al Layered Double Hydroxides for Ammonia Decomposition. Chem. Commun. 2019, 55 (96), 14410-14413.
(213) Hayashi, F.; Toda, Y.; Kanie, Y.; Kitano, M.; Inoue, Y.; Yokoyama, T.; Hara, M.; Hosono, H. Ammonia Decomposition by Ruthenium Nanoparticles Loaded on Inorganic Electride C12A7: $\mathrm{E}^{-}$. Chem. Sci. 2013, 4 (8), 3124-3130.

(214) Wang, Z.; Cai, Z.; Wei, Z. Highly Active Ruthenium Catalyst Supported on Barium Hexaaluminate for Ammonia Decomposition to $\mathrm{CO}_{\mathrm{x}}$-Free Hydrogen. ACS Sustainable Chem. Eng. 2019, 7 (9), 82268235.

(215) Nagaoka, K.; Eboshi, T.; Abe, N.; Miyahara, S. I.; Honda, K.; Sato, K. Influence of Basic Dopants on the Activity of $\mathrm{Ru} / \operatorname{Pr}_{6} \mathrm{O}_{11}$ for Hydrogen Production by Ammonia Decomposition. Int. J. Hydrogen Energy 2014, 39 (35), 20731-20735.

(216) Nagaoka, K.; Honda, K.; Ibuki, M.; Sato, K.; Takita, Y. Highly Active $\mathrm{Cs}_{2} \mathrm{O} / \mathrm{Ru} / \mathrm{Pr}_{6} \mathrm{O}_{11}$ as a Catalyst for Ammonia Decomposition. Chem. Lett. 2010, 39 (9), 918-919.

(217) Zheng, W.; Zhang, J.; Xu, H.; Li, W. $\mathrm{NH}_{3}$ Decomposition Kinetics on Supported Ru Clusters: Morphology and Particle Size Effect. Catal. Lett. 2007, 119 (3-4), 311-318.

(218) Simonsen, S. B.; Chakraborty, D.; Chorkendorff, I.; Dahl, S. Alloyed Ni-Fe Nanoparticles as Catalysts for $\mathrm{NH}_{3}$ Decomposition. Appl. Catal., A 2012, 447-448, 22-31.

(219) Lucentini, I.; Casanovas, A.; Llorca, J. Catalytic Ammonia Decomposition for Hydrogen Production on $\mathrm{Ni}, \mathrm{Ru}$ and $\mathrm{Ni}-\mathrm{Ru}$ Supported on $\mathrm{CeO}_{2}$. Int. J. Hydrogen Energy 2019, 44, 12693-12707.

(220) Prasad, V.; Karim, A. M.; Arya, A.; Vlachos, D. G. Assessment of Overall Rate Expressions and Multiscale, Microkinetic Model Uniqueness via Experimental Data Injection: Ammonia Decomposition on $\mathrm{Ru} / \gamma-\mathrm{Al}_{2} \mathrm{O}_{3}$ for Hydrogen Production. Ind. Eng. Chem. Res. 2009, 48 (11), 5255-5265.

(221) Karim, A. M.; Prasad, V.; Mpourmpakis, G.; Lonergan, W. W.; Frenkel, A. I.; Chen, J. G.; Vlachos, D. G. Correlating Particle Size and Shape of Supported $\mathrm{Ru} / \gamma-\mathrm{Al}_{2} \mathrm{O}_{3}$ Catalysts with $\mathrm{NH}_{3}$ Decomposition Activity. J. Am. Chem. Soc. 2009, 131 (34), 12230-12239.

(222) McCullough, K.; Chiang, P.-H.; Jimenez, J. D.; Lauterbach, J. A. Material Discovery and High Throughput Exploration of Ru Based Catalysts for Low Temperature Ammonia Decomposition. Materials 2020, 13 (8), 1-19.

(223) Su, Q.; Gu, L.; Yao, Y.; Zhao, J.; Ji, W.; Ding, W.; Au, C.-T. Layered Double Hydroxides Derived $\mathrm{Ni}_{\mathrm{x}}\left(\mathrm{Mg}_{\mathrm{y}} \mathrm{Al}_{\mathrm{z}} \mathrm{O}_{\mathrm{n}}\right)$ Catalysts: Enhanced Ammonia Decomposition by Hydrogen Spillover Effect. Appl. Catal., B 2017, 201, 451-460.

(224) Bramwell, P. L.; Lentink, S.; Ngene, P.; De Jongh, P. E. Effect of Pore Confinement of $\mathrm{LiNH}_{2}$ on Ammonia Decomposition Catalysis and the Storage of Hydrogen and Ammonia. J. Phys. Chem. C 2016, 120 (48), 27212-27220.

(225) Lamb, K.; Hla, S. S.; Dolan, M. Ammonia Decomposition Kinetics over LiOH-Promoted, $\alpha-\mathrm{Al}_{2} \mathrm{O}_{3}$-Supported Ru Catalyst. Int. J. Hydrogen Energy 2019, 44 (7), 3726-3736.

(226) Torrente-Murciano, L.; Hill, A. K.; Bell, T. E. Ammonia Decomposition over Cobalt/Carbon Catalysts-Effect of Carbon Support and Electron Donating Promoter on Activity. Catal. Today 2017, 286, 131-140.

(227) Raróg-Pilecka, W.; Szmigiel, D.; Komornicki, A.; Zieliński, J.; Kowalczyk, Z. Catalytic Properties of Small Ruthenium Particles Deposited on Carbon: Ammonia Decomposition Studies. Carbon 2003, 41 (3), 589-591.

(228) Xie, P.; Yao, Y.; Huang, Z.; Liu, Z.; Zhang, J.; Li, T.; Wang, G.; Shahbazian-Yassar, R.; Hu, L.; Wang, C. Highly Efficient Decomposition of Ammonia Using High-Entropy Alloy Catalysts. Nat. Commun. 2019, 10 (4011), 1-12.

(229) Wang, L.; Chen, J.; Ge, L.; Rudolph, V.; Zhu, Z. Difference in the Cooperative Interaction between Carbon Nanotubes and $\mathrm{Ru}$ Particles Loaded on Their Internal/External Surface. RSC Adv. 2013, 3 (31), 12641-12647.

(230) Zheng, W.; Zhang, J.; Zhu, B.; Blume, R.; Zhang, Y.; Schlichte, K.; Schlögl, R.; Schüth, F.; Su, D. S. Structure-Function Correlations for $\mathrm{Ru} / \mathrm{CNT}$ in the Catalytic Decomposition of Ammonia. ChemSusChem 2010, 3 (2), 226-230. 
(231) Chen, C.; Chen, Y.; Ali, A. M.; Luo, W.; Wen, J.; Zhang, L.; Zhang, H. Bimetallic Ru-Fe Nanoparticles Supported on Carbon Nanotubes for Ammonia Decomposition and Synthesis. Chem. Eng. Technol. 2020, 43 (4), 719-730.

(232) Guo, J.; Chen, Z.; Wu, A.; Chang, F.; Wang, P.; Hu, D.; Wu, G.; Xiong, Z.; Yu, P.; Chen, P. Electronic Promoter or Reacting Species? The Role of $\mathrm{LiNH}_{2}$ on $\mathrm{Ru}$ in Catalyzing $\mathrm{NH}_{3}$ Decomposition. Chem. Commun. 2015, 51 (82), 15161-15164.

(233) Guo, J.; Wang, P.; Wu, G.; Wu, A.; Hu, D.; Xiong, Z.; Wang, J.; Yu, P.; Chang, F.; Chen, Z.; Chen, P. Lithium Imide Synergy with 3d Transition-Metal Nitrides Leading to Unprecedented Catalytic Activities for Ammonia Decomposition. Angew. Chem. 2015, 127 (10), 2993-2997.

(234) Li, L.; Wang, Y.; Xu, Z. P.; Zhu, Z. Catalytic Ammonia Decomposition for CO-Free Hydrogen Generation over $\mathrm{Ru} / \mathrm{Cr}_{2} \mathrm{O}_{3}$ Catalysts. Appl. Catal., A 2013, 467, 246-252.

(235) Chang, F.; Wu, H.; Van der Pluijm, R.; Guo, J.; Ngene, P.; De Jongh, P. E. Effect of Pore Confinement of $\mathrm{NaNH}_{2}$ and $\mathrm{KNH}_{2}$ on Hydrogen Generation from Ammonia. J. Phys. Chem. C 2019, 123 (35), 21487-21496.

(236) Zheng, W.; Cotter, T. P.; Kaghazchi, P.; Jacob, T.; Frank, B.; Schlichte, K.; Zhang, W.; Su, D. S.; Schüth, F.; Schlögl, R. Experimental and Theoretical Investigation of Molybdenum Carbide and Nitride as Catalysts for Ammonia Decomposition. J. Am. Chem. Soc. 2013, 135 (9), 3458-3464.

(237) Yu, P.; Guo, J.; Liu, L.; Wang, P.; Chang, F.; Wang, H.; Ju, X.; Chen, P. Effects of Alkaline Earth Metal Amides on Ru in Catalytic Ammonia Decomposition. J. Phys. Chem. C 2016, 120 (5), 2822-2828.

(238) Takahashi, A.; Fujitani, T. Kinetic Analysis of Decomposition of Ammonia over Nickel and Ruthenium Catalysts. J. Chem. Eng. Jpn. 2016, 49 (1), 22-28.

(239) Maeda, A.; Hu, Z.; Kunimori, K.; Uchijima, T. Effect of HighTemperature Reduction on Ammonia Decomposition over NiobiaSupported and Nioba-Promoted Rhodium Catalysts. Catal. Lett. 1988, $1,155-157$.

(240) Richardson, D. J.; Hellgardt, K.; Russell, P. A.; Mason, G.; Buffman, B. A. Flux Response Analysis: A Study of Ammonia Decomposition over Pt/Alumina. Chem. Eng. Res. Des. 2004, 82 (10), 1397-1403.

(241) Polański, J.; Bartczak, P.; Ambrozkiewicz, W.; Sitko, R.; Siudyga, T.; Mianowski, A.; Szade, J.; Balin, K.; Lelątko, J. Ni-Supported Pd Nanoparticles with Ca Promoter: A New Catalyst for Low-Temperature Ammonia Cracking. PLoS One 2015, 10 (8), e0136805.

(242) Choi, J.-G. Ammonia Decomposition over Vanadium Carbide Catalysts. J. Catal. 1999, 182 (1), 104-116.

(243) Varışl1, D.; Rona, T. $\mathrm{CO}_{\mathrm{x}}$ Free Hydrogen Production from Ammonia Decomposition over Platinum Based Siliceous Materials. Int. J. Chem. React. Eng. 2012, 10 (1), 1-30.

(244) Donald, J.; Xu, C.; Hashimoto, H.; Byambajav, E.; Ohtsuka, Y. Novel Carbon-Based Ni/Fe Catalysts Derived from Peat for Hot Gas Ammonia Decomposition in an Inert Helium Atmosphere. Appl. Catal, A 2010, 375 (1), 124-133.

(245) Gu, Y.-Q.; Jin, Z.; Zhang, H.; Xu, R.-J.; Zheng, M.-J.; Guo, Y.M.; Song, Q.-S.; Jia, C.-J. Transition Metal Nanoparticles Dispersed in an Alumina Matrix as Active and Stable Catalysts for $\mathrm{CO}_{\mathrm{x}}$-Free Hydrogen Production from Ammonia. J. Mater. Chem. A 2015, 3 (33), $17172-17180$.

(246) Hu, X.-C.; Wang, W.-W.; Jin, Z.; Wang, X.; Si, R.; Jia, C.-J. Transition Metal Nanoparticles Supported La-Promoted $\mathrm{MgO}$ as Catalysts for Hydrogen Production via Catalytic Decomposition of Ammonia. J. Energy Chem. 2019, 38, 41-49.

(247) Zhang, H.; Alhamed, Y. A.; Kojima, Y.; Al-Zahrani, A. A.; Petrov, L. A. Cobalt Supported on Carbon Nanotubes. An Efficient Catalyst for Ammonia Decomposition. C. R. Acad. Bulg. Sci. 2013, 66 (4), 519-524.

(248) Xun, Y.; He, X.; Yan, H.; Gao, Z.; Jin, Z.; Jia, C. Fe- and CoDoped Lanthanum Oxides Catalysts for Ammonia Decomposition: Structure and Catalytic Performances. J. Rare Earths 2017, 35 (1), 1523.
(249) Xu, C.; Tsubouchi, N.; Hashimoto, H.; Ohtsuka, Y. Catalytic Decomposition of Ammonia Gas with Metal Cations Present Naturally in Low Rank Coals. Fuel 2005, 84 (14-15), 1957-1967.

(250) Dasireddy, V. D. B. C.; Likozar, B. $\mathrm{CO}_{\mathrm{x}}$-Free Hydrogen Generation via Decomposition of Ammonia over Copper and ZincBased Catalysts. Fuel 2017, 196, 325-335.

(251) Zhang, J.; Xu, H.; Li, W. Kinetic Study of $\mathrm{NH}_{3}$ Decomposition over Ni Nanoparticles: The Role of La Promoter, Structure Sensitivity and Compensation Effect. Appl. Catal., A 2005, 296 (12), 257-267.

(252) Zhang, J.; Xu, H.; Jin, X.; Ge, Q.; Li, W. Characterizations and Activities of the Nano-Sized $\mathrm{Ni} / \mathrm{Al}_{2} \mathrm{O}_{3}$ and $\mathrm{Ni} / \mathrm{La}-\mathrm{Al}_{2} \mathrm{O}_{3}$ Catalysts for $\mathrm{NH}_{3}$ Decomposition. Appl. Catal., A 2005, 290 (1-2), 87-96.

(253) Yan, H.; Xu, Y.-J.; Gu, Y.-Q.; Li, H.; Wang, X.; Jin, Z.; Shi, S.; Si, R.; Jia, C.-J.; Yan, C.-H. Promoted Multimetal Oxide Catalysts for the Generation of Hydrogen via Ammonia Decomposition. J. Phys. Chem. C 2016, 120 (14), 7685-7696.

(254) Okura, K.; Okanishi, T.; Muroyama, H.; Matsui, T.; Eguchi, K. Ammonia Decomposition over Nickel Catalysts Supported on RareEarth Oxides for the On-Site Generation of Hydrogen. Chem CatChem 2016, 8, 2988-2995.

(255) Nakamura, I.; Fujitani, T. Role of Metal Oxide Supports in $\mathrm{NH}_{3}$ Decomposition over Ni. Appl. Catal., A 2016, 524, 45-49.

(256) Muroyama, H.; Saburi, C.; Matsui, T.; Eguchi, K. Ammonia Decomposition over $\mathrm{Ni} / \mathrm{La}_{2} \mathrm{O}_{3}$ Catalyst for on-Site Generation of Hydrogen. Appl. Catal., A 2012, 443-444, 119-124.

(257) Okura, K.; Okanishi, T.; Muroyama, H.; Matsui, T.; Eguchi, K. Promotion Effect of Rare-Earth Elements on the Catalytic Decomposition of Ammonia over $\mathrm{Ni} / \mathrm{Al}_{2} \mathrm{O}_{3}$ Catalyst. Appl. Catal., A 2015, 505, $77-85$.

(258) Okura, K.; Miyazaki, K.; Muroyama, H.; Matsui, T.; Eguchi, K. Ammonia Decomposition over Ni Catalysts Supported on PerovskiteType Oxides for the on-Site Generation of Hydrogen. RSC Adv. 2018, 8 (56), 32102-32110.

(259) Liu, Y.; Wang, H.; Li, J.; Lu, Y.; Wu, H.; Xue, Q.; Chen, L. Monolithic Microfibrous Nickel Catalyst Co-Modified with Ceria and Alumina for Miniature Hydrogen Production via Ammonia Decomposition. Appl. Catal., A 2007, 328 (1), 77-82.

(260) Zheng, W.; Zhang, J.; Ge, Q.; Xu, H.; Li, W. Effects of $\mathrm{CeO}_{2}$ Addition on $\mathrm{Ni} / \mathrm{Al}_{2} \mathrm{O}_{3}$ Catalysts for the Reaction of Ammonia Decomposition to Hydrogen. Appl. Catal., B 2008, 80 (1-2), 98-105.

(261) Vacharapong, P.; Arayawate, S.; Henpraserttae, S.; Katanyutanon, S.; Charojrochkul, S.; Lawtrakul, L.; Toochinda, P. Effect of Magnetic Inducement in Preparation of Ni/Ce-Doped $\mathrm{Al}_{2} \mathrm{O}_{3}$ for Ammonia Decomposition. ChemistrySelect 2019, 4 (40), 1191311919.

(262) Liu, H.; Wang, H.; Shen, J.; Sun, Y.; Liu, Z. Promotion Effect of Cerium and Lanthanum Oxides on Ni/SBA-15 Catalyst for Ammonia Decomposition. Catal. Today 2008, 131 (1-4), 444-449.

(263) Trovarelli, A.; Llorca, J. Ceria Catalysts at Nanoscale: How Do Crystal Shapes Shape Catalysis? ACS Catal. 2017, 7 (7), 4716-4735.

(264) Trovarelli, A. Catalytic Properties of Ceria and $\mathrm{CeO}_{2}$ Containing Materials. Catal. Rev.: Sci. Eng. 1996, 38 (4), 439-520.

(265) Campbell, C. T.; Peden, C. H. F. Oxygen Vacancies and Catalysis on Ceria Surfaces. Science (Washington, DC, U. S.) 2005, 309 (5735), 713-714.

(266) Melchionna, M.; Fornasiero, P. The Role of Ceria-Based Nanostructured Materials in Energy Applications. Mater. Today 2014, 17 (7), 349-357.

(267) Choudhary, T. V.; Sivadinarayana, C.; Goodman, D. W. Production of $\mathrm{CO}_{\mathrm{x}}$-Free Hydrogen for Fuel Cells via Step-Wise Hydrocarbon Reforming and Catalytic Dehydrogenation of Ammonia. Chem. Eng. J. 2003, 93 (1), 69-80.

(268) Atsumi, R.; Noda, R.; Takagi, H.; Vecchione, L.; Di Carlo, A.; Del Prete, Z.; Kuramoto, K. Ammonia Decomposition Activity over $\mathrm{Ni} / \mathrm{SiO}_{2}$ Catalysts with Different Pore Diameters. Int. J. Hydrogen Energy 2014, 39 (26), 13954-13961.

(269) Li, Y.; Wen, J.; Ali, A. M.; Duan, M.; Zhu, W.; Zhang, H.; Chen, C.; Li, Y. Size Structure - Catalytic Performance Correlation of 
Supported Ni/MCF-17 Catalysts for $\mathrm{CO}_{\mathrm{x}}$-Free Hydrogen Production. Chem. Commun. 2018, 54, 6364-6367.

(270) Duan, X.; Qian, G.; Liu, Y.; Ji, J.; Zhou, X.; Chen, D.; Yuan, W. Structure Sensitivity of Ammonia Decomposition over Ni Catalysts: A Computational and Experimental Study. Fuel Process. Technol. 2013, $108,112-117$.

(271) Zhang, L.-F.; Li, M.; Ren, T.-Z.; Liu, X.; Yuan, Z.-Y. CeModified $\mathrm{Ni}$ Nanoparticles Encapsulated in $\mathrm{SiO}_{2}$ for $\mathrm{CO}_{\mathrm{x}}$-Free Hydrogen Production via Ammonia Decomposition. Int. J. Hydrogen Energy 2015, 40 (6), 2648-2656.

(272) Liu, H.; Wang, H.; Shen, J.; Sun, Y.; Liu, Z. Preparation, Characterization and Activities of the Nano-Sized Ni/SBA-15 Catalyst for Producing $\mathrm{CO}_{\mathrm{x}}$-Free Hydrogen from Ammonia. Appl. Catal., A 2008, 337 (2), 138-147.

(273) Li, L.; Chen, F.; Shao, J.; Dai, Y.; Ding, J.; Tang, Z. Attapulgite Clay Supported Ni Nanoparticles Encapsulated by Porous Silica: Thermally Stable Catalysts for Ammonia Decomposition to $\mathrm{CO}_{\mathrm{x}}$ Free Hydrogen. Int. J. Hydrogen Energy 2016, 41 (46), 21157-21165.

(274) Kurtoğlu, S. F.; Sarp, S.; Yılmaz Akkaya, C.; Yağc1, B.; Motallebzadeh, A.; Soyer-Uzun, S.; Uzun, A. $\mathrm{CO}_{\mathrm{x}}$-Free Hydrogen Production from Ammonia Decomposition over Sepiolite-Supported Nickel Catalysts. Int. J. Hydrogen Energy 2018, 43 (21), 9954-9968.

(275) Henpraserttae, S.; Leong, H. W.; Ratprasatpon, P.; Moolgate, J.; Toochinda, P. Effect of Acidic Sites of Support to Nickle Catalysts for Ammonia Decomposition. 2016 2nd Asian Conference on Defence Technology (ACDT) 2016, 167-169.

(276) Henpraserttae, S.; Charojrochkul, S.; Klysubun, W.; Lawtrakul, L.; Toochinda, P. Reduced Temperature Ammonia Decomposition Using $\mathrm{Ni} / \mathrm{Zr}$-Doped $\mathrm{Al}_{2} \mathrm{O}_{3}$ Catalyst. Catal. Lett. 2018, 148 (6), 17751783.

(277) Henpraserttae, S.; Charojrochkul, S.; Lawtrakul, L.; Toochinda, P. Ni-Based Catalysts for Hydrogen Production from Ammonia Decomposition: Effect of Dopants and Urine Application. ChemistrySelect 2018, 3 (42), 11842-11850.

(278) Deng, Q.-F.; Zhang, H.; Hou, X.-X.; Ren, T.-Z.; Yuan, Z.-Y. High-Surface-Area $\mathrm{Ce}_{0.8} \mathrm{Zr}_{0.2} \mathrm{O}_{2}$ Solid Solutions Supported Ni Catalysts for Ammonia Decomposition to Hydrogen. Int. J. Hydrogen Energy 2012, 37 (21), 15901-15907.

(279) Sima, D.; Wu, H.; Tian, K.; Xie, S.; Foo, J. J.; Li, S.; Wang, D.; Ye, Y.; Zheng, Z.; Liu, Y. Q. Enhanced Low Temperature Catalytic Activity of $\mathrm{Ni} / \mathrm{Al}-\mathrm{Ce}_{0.8} \mathrm{Zr}_{0.2} \mathrm{O}_{2}$ for Hydrogen Production from Ammonia Decomposition. Int. J. Hydrogen Energy 2020, 45 (16), 9342-9352.

(280) Zhao, J.; Deng, L.; Zheng, W.; Xu, S.; Yu, Q.; Qiu, X. NickelInduced Structure Transformation in Hydrocalumite for Enhanced Ammonia Decomposition. Int. J. Hydrogen Energy 2020, 45 (22), 12244-12255.

(281) Sato, K.; Abe, N.; Kawagoe, T.; Miyahara, S.; Honda, K.; Nagaoka, K. Supported Ni Catalysts Prepared from Hydrotalcite-like Compounds for the Production of Hydrogen by Ammonia Decomposition. Int. J. Hydrogen Energy 2017, 42 (10), 6610-6617.

(282) Inokawa, H.; Ichikawa, T.; Miyaoka, H. Catalysis of Nickel Nanoparticles with High Thermal Stability for Ammonia Decomposition. Appl. Catal., A 2015, 491, 184-188.

(283) Alhamed, Y. A.; Zhang, H.; Kojima, Y.; Al-Zahrani, A. A.; Hafedh, D.; Petrov, L. A. Effect of Surface Functional Groups Attached to Carbon Nanotubes Used as Support of Nickel Catalysts on Their Structure and Catalytic Performance for Ammonia Decomposition. Comptes Rendus L'Academie Bulg. des Sci. 2014, 67 (4), 519-526.

(284) Zhang, H.; Alhamed, Y. A.; Kojima, Y.; Al-Zahrani, A. A.; Miyaoka, H.; Petrov, L. A. Structure and Catalytic Properties of Ni/ MWCNTs and Ni/AC Catalysts for Hydrogen Production via Ammonia Decomposition. Int. J. Hydrogen Energy 2014, 39 (1), 277-287.

(285) Meng, T.; Xu, Q.-Q.; Li, Y.-T.; Chang, J.-L.; Ren, T.-Z.; Yuan, Z.-Y. Nickle Nanoparticles Highly Dispersed on Reduced Graphene Oxide for Ammonia Decomposition to Hydrogen. J. Ind. Eng. Chem. 2015, 32, 373-379.
(286) Cao, J. L.; Yan, Z.-L.; Deng, Q.-F.; Yuan, Z.-Y.; Wang, Y.; Sun, G.; Wang, X.-D.; Hari, B.; Zhang, Z.-Y. Homogeneous Precipitation Method Preparation of Modified Red Mud Supported Ni Mesoporous Catalysts for Ammonia Decomposition. Catal. Sci. Technol. 2014, 4 (2), 361-368.

(287) Cao, J.-L.; Yan, Z.-L.; Deng, Q.-F.; Wang, Y.; Yuan, Z.-Y.; Sun, G.; Jia, T.-K.; Wang, X.-D.; Bala, H.; Zhang, Z.-Y. Mesoporous Modified-Red-Mud Supported Ni Catalysts for Ammonia Decomposition to Hydrogen. Int. J. Hydrogen Energy 2014, 39 (11), 57475755.

(288) Lu, A.-H.; Nitz, J.-J.; Comotti, M.; Weidenthaler, C.; Schlichte, K.; Lehmann, C. W.; Terasaki, O.; Schüth, F. Spatially and Size Selective Synthesis of Fe-Based Nanoparticles on Ordered Mesoporous Supports as Highly Active and Stable Catalysts for Ammonia Decomposition. J. Am. Chem. Soc. 2010, 132 (40), 14152-14162.

(289) Tüysüz, H.; Schüth, F.; Zhi, L.; Müllen, K.; Comotti, M. Ammonia Decomposition over Iron Phthalocyanine-Based Materials. ChemCatChem 2015, 7 (9), 1453-1459.

(290) Liang, C.; Li, W.; Wei, Z.; Xin, Q.; Li, C. Catalytic

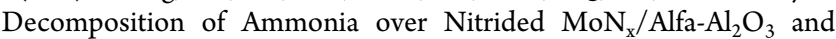
$\mathrm{NiMoN}_{\mathrm{y}} / \mathrm{Alfa}_{-} \mathrm{Al}_{2} \mathrm{O}_{3}$ Catalysts. Ind. Eng. Chem. Res. 2000, 39, 36943697.

(291) Han, X.; Chu, W.; Ni, P.; Luo, S. Z.; Zhang, T. Promoting Effects of Iridium on Nickel Based Catalyst in Ammonia Decomposition. J. Fuel Chem. Technol. 2007, 35 (6), 691-695.

(292) Huang, C.; Li, H.; Yang, J.; Wang, C.; Hu, F.; Wang, X.; Lu, Z. H.; Feng, G.; Zhang, R. Ce ${ }_{0.6} \mathrm{Zr}_{0.3} \mathrm{Y}_{0.1} \mathrm{O}_{2}$ Solid Solutions-Supported NiCo Bimetal Nanocatalysts for $\mathrm{NH}_{3}$ Decomposition. Appl. Surf. Sci. 2019, 478, 708-716.

(293) Hu, Z.-P.; Weng, C.-C.; Chen, C.; Yuan, Z.-Y. Catalytic Decomposition of Ammonia to $\mathrm{CO}_{\mathrm{x}}$-Free Hydrogen over Ni/ZSM-5 Catalysts: A Comparative Study of the Preparation Methods. Appl. Catal., A 2018, 562, 49-57.

(294) Kunsman, C. H. The Decomposition of Ammonia on Iron Catalysts. Science (Washington, DC, U. S.) 1927, 65 (1691), 527-528.

(295) Kowalczyk, Z.; Sentek, J.; Jodzis, S.; Muhler, M.; Hinrichsen, O. Effect of Potassium on the Kinetics of Ammonia Synthesis and Decomposition over Fused Iron Catalyst at Atmospheric Pressure. J. Catal. 1997, 169, 407-414.

(296) Arabczyk, W.; Pelka, R. Studies of the Kinetics of Two Parallel Reactions: Ammonia Decomposition and Nitriding of Iron Catalyst. J. Phys. Chem. A 2009, 113 (2), 411-416.

(297) Othman, N. E. F.; Salleh, H. M.; Purwanto, H. Utilization of Low-Grade Iron Ore in Ammonia Decomposition. Procedia Chem. 2016, 19, 119-124.

(298) Tseng, J.-C.; Gu, D.; Pistidda, C.; Horstmann, C.; Dornheim, M.; Ternieden, J.; Weidenthaler, C. Tracking the Active Catalyst for Iron-Based Ammonia Decomposition by In Situ Synchrotron Diffraction Studies. ChemCatChem 2018, 10 (19), 4465.

(299) Pelka, R.; Moszyńska, I.; Arabczyk, W. Catalytic Ammonia Decomposition over $\mathrm{Fe} / \mathrm{Fe}_{4} \mathrm{~N}$. Catal. Lett. 2009, 128 (1-2), 72-76.

(300) Pelka, R.; Arabczyk, W. Studies of the Kinetics of Reaction between Iron Catalysts and Ammonia-Nitriding of Nanocrystalline Iron with Parallel Catalytic Ammonia Decomposition. Top. Catal. 2009, 52 (11), 1506-1516.

(301) Pelka, R.; Kiełbasa, K.; Arabczyk, W. Catalytic Ammonia Decomposition during Nanocrystalline Iron Nitriding at $475^{\circ} \mathrm{C}$ with $\mathrm{NH}_{3} / \mathrm{H}_{2}$ Mixtures of Different Nitriding Potentials. J. Phys. Chem. C 2014, 118 (12), 6178-6185.

(302) Kiełbasa, K.; Pelka, R.; Arabczyk, W. Studies of the Kinetics of Ammonia Decomposition on Promoted Nanocrystalline Iron Using Gas Phases of Different Nitriding Degree. J. Phys. Chem. A 2010, 114 (13), 4531-4534.

(303) Pelka, R.; Kiełbasa, K.; Arabczyk, W. The Effect of Iron Nanocrystallites' Size in Catalysts for Ammonia Synthesis on Nitriding Reaction and Catalytic Ammonia Decomposition. Cent. Eur. J. Chem. 2011, 9 (2), 240-244.

(304) Pelka, R.; Arabczyk, W. Influence of Chemical Composition of Nanocrystalline Iron's Surface on the Rates of Two Parallel Reactions: 
Nitriding and Catalytic Decomposition of Ammonia. Chem. Pap. 2012, 66 (1), 18-25.

(305) Feyen, M.; Weidenthaler, C.; Guttel, R.; Schlichte, K.; Holle, U.; Lu, A.-H.; Schuth, F. Schüth, F. High-Temperature Stable, Iron-Based Core-Shell Catalysts for Ammonia Decomposition. Chem. - Eur. J. 2011, 17 (2), 598-605.

(306) Li, Y.; Yao, L.; Liu, S.; Zhao, J.; Ji, W.; Au, C.-T. Cs-Modified Iron Nanoparticles Encapsulated in Microporous and Mesoporous $\mathrm{SiO}_{2}$ for $\mathrm{CO}_{\mathrm{x}}$-Free $\mathrm{H}_{2}$ Production via Ammonia Decomposition. Catal. Today 2011, 160 (1), 79-86.

(307) Hu, Z.-P.; Chen, L.; Chen, C.; Yuan, Z.-Y. Fe/ZSM-5 Catalysts for Ammonia Decomposition to $\mathrm{CO}_{\mathrm{x}}$-Free Hydrogen: Effect of $\mathrm{SiO}_{2}$ / $\mathrm{Al}_{2} \mathrm{O}_{3}$ Ratio. Mol. Catal. 2018, 455, 14-22.

(308) Cui, H.-Z.; Gu, Y.-Q.; He, X.-X.; Wei, S.; Jin, Z.; Jia, C.-J.; Song, Q.-S. Iron-Based Composite Nanostructure Catalysts Used to Produce $\mathrm{CO}_{\mathrm{x}}$-Free Hydrogen from Ammonia. Sci. Bull. 2016, 61 (3), 220-226.

(309) Tsubouchi, N.; Hashimoto, H.; Ohtsuka, Y. High Catalytic Performance of Fine Particles of Metallic Iron Formed from Limonite in the Decomposition of a Low Concentration of Ammonia. Catal. Lett. 2005, 105 (3-4), 203-208.

(310) Rein, D.; Friedel Ortega, K.; Weidenthaler, C.; Bill, E.; Behrens, M. The Roles of Co-Precipitation PH, Phase-Purity and Alloy Formation for the Ammonia Decomposition Activity of Ga-Promoted $\mathrm{Fe} / \mathrm{MgO}$ Catalysts. Appl. Catal., A 2017, 548, 52-61.

(311) Ortega, K. F.; Rein, D.; Lüttmann, C.; Heese, J.; Özcan, F.; Heidelmann, M.; Folke, J.; Kähler, K.; Schlögl, R.; Behrens, M. Ammonia Decomposition and Synthesis over Multinary Magnesioferrites: Promotional Effect of $\mathrm{Ga}$ on $\mathrm{Fe}$ Catalysts for the Decomposition Reaction. ChemCatChem 2017, 9 (4), 659-671.

(312) Itoh, M.; Masuda, M.; Machida, K. Hydrogen Generation by Ammonia Cracking with Iron Metal-Rare Earth Oxide Composite Catalyst. Mater. Trans. 2002, 43 (11), 2763-2767.

(313) Li, L.; Meng, Q.; Ji, W.; Shao, J.; Xu, Q.; Yan, J. Embedded Iron Nanoparticles by Graphitized Carbon as Highly Active yet Stable Catalyst for Ammonia Decomposition. Mol. Catal. 2017, 442, 147153.

(314) Jedynak, A.; Kowalczyk, Z.; Szmigiel, D.; Rarog, W.; Zielinski, J. Ammonia Decomposition over the Carbon-Based Iron Catalyst Promoted with Potassium. Appl. Catal., A 2002, 237, 223-226.

(315) Ji, J.; Duan, X.; Qian, G.; Li, P.; Zhou, X.; Chen, D.; Yuan, W. Fe Particles on the Tops of Carbon Nanofibers Immobilized on Structured Carbon Microfibers for Ammonia Decomposition. Catal. Today 2013, 216, 254-260.

(316) Ji, J.; Yan, X.; Qian, G.; Peng, C.; Duan, X.; Zhou, X. Morphology and Location Manipulation of Fe Nanoparticles on Carbon Nanofibers as Catalysts for Ammonia Decomposition to Generate Hydrogen. Int. J. Hydrogen Energy 2017, 42 (27), 1746617475 .

(317) Duan, X.; Qian, G.; Zhou, X.; Sui, Z.; Chen, D.; Yuan, W. Tuning the Size and Shape of Fe Nanoparticles on Carbon Nanofibers for Catalytic Ammonia Decomposition. Appl. Catal., B 2011, 101 (34), 189-196.

(318) Wang, Y.; Ross Kunz, M.; Siebers, S.; Rollins, H. W.; Gleaves, J.; Yablonsky, G.; Fushimi, R. Transient Kinetic Experiments within the High Conversion Domain: The Case of Ammonia Decomposition. Catalysts 2019, 9 (1), 1-19.

(319) Otremba, T.; Frenzel, N.; Lerch, M.; Ressler, T.; Schomäcker, R. Kinetic Studies on Ammonia Decomposition over Zirconium Oxynitride. Appl. Catal., A 2011, 392 (1-2), 103-110.

(320) Vilekar, S. a.; Fishtik, I.; Datta, R. The Peculiar Catalytic Sequence of the Ammonia Decomposition Reaction and Its SteadyState Kinetics. Chem. Eng. Sci. 2012, 71, 333-344.

(321) Arabczyk, W.; Zamlynny, J. Study of the Ammonia Decomposition over Iron Catalysts. Catal. Lett. 1999, 60, 167-171.

(322) Zhang, J.; Muller, J.-O.; Zheng, W.; Wang, D.; Su, D.; Schlogl, R. Individual $\mathrm{Fe}-\mathrm{Co}$ Alloy Nanoparticles on Carbon Nanotubes: Structural and Catalytic Properties. Nano Lett. 2008, 8 (9), 2738-2743.
(323) Lorenzut, B.; Montini, T.; Bevilacqua, M.; Fornasiero, P. FeMoBased Catalysts for $\mathrm{H}_{2}$ Production by $\mathrm{NH}_{3}$ Decomposition. Appl. Catal., B 2012, 125, 409-417.

(324) Lendzion-Bieluń, Z.; Pelka, R.; Arabczyk, W. Study of the Kinetics of Ammonia Synthesis and Decomposition on Iron and Cobalt Catalysts. Catal. Lett. 2009, 129 (1-2), 119-123.

(325) Zhang, Z.-S.; Fu, X. P.; Wang, W.-W.; Jin, Z.; Song, Q.-S.; Jia, C.-J. Promoted Porous $\mathrm{Co}_{3} \mathrm{O}_{4}-\mathrm{Al}_{2} \mathrm{O}_{3}$ Catalysts for Ammonia Decomposition. Sci. China: Chem. 2018, 61 (11), 1389-1398.

(326) Czekajło, Ł.; Lendzion-Bieluń, Z. Effect of Preparation Conditions and Promoters on the Structure and Activity of the Ammonia Decomposition Reaction Catalyst Based on Nanocrystalline Cobalt. Chem. Eng. J. 2016, 289, 254-260.

(327) Gu, Y.-Q.; Fu, X.-P.; Du, P.-P.; Gu, D.; Jin, Z.; Huang, Y.-Y.; Si, R.; Zheng, L.-Q.; Song, Q.-S.; Jia, C.-J.; Weidenthaler, C. In Situ X-Ray Diffraction Study of Co-Al Nanocomposites as Catalysts for Ammonia Decomposition. J. Phys. Chem. C 2015, 119 (30), 17102-17110.

(328) Zhang, H.; Alhamed, Y. A.; Chu, W.; Ye, Z.; Alzahrani, A.; Petrov, L. Controlling Co-Support Interaction in Co/MWCNTs Catalysts and Catalytic Performance for Hydrogen Production via $\mathrm{NH}_{3}$ Decomposition. Appl. Catal., A 2013, 464-465, 156-164.

(329) Zhang, H.; Alhamed, Y. A.; Al-Zahrani, A.; Daous, M.; Inokawa, H.; Kojima, Y.; Petrov, L. A. Tuning Catalytic Performances of Cobalt Catalysts for Clean Hydrogen Generation via Variation of the Type of Carbon Support and Catalyst Post-Treatment Temperature. Int. J. Hydrogen Energy 2014, 39 (31), 17573-17582.

(330) Lara-García, H. A.; Mendoza-Nieto, J. A.; Pfeiffer, H.; TorrenteMurciano, L. $\mathrm{CO}_{\mathrm{x}}$-Free Hydrogen Production from Ammonia on Novel Cobalt Catalysts Supported on 1D Titanate Nanotubes. Int. J. Hydrogen Energy 2019, 44 (57), 30062-30074.

(331) Podila, S.; Alhamed, Y. A.; Alzahrani, A. A.; Petrov, L. A. Hydrogen Production by Ammonia Decomposition Using Co Catalyst Supported on Mg Mixed Oxide Systems. Int. J. Hydrogen Energy 2015, 40 (45), 15411-15422.

(332) Podila, S.; Driss, H.; Zaman, S. F.; Alhamed, Y. A.; Alzahrani, A. A.; Daous, M. A.; Petrov, L. A. Hydrogen Generation by Ammonia Decomposition Using $\mathrm{Co} / \mathrm{MgO}-\mathrm{La}_{2} \mathrm{O}_{3}$ Catalyst: Influence of Support Calcination Atmosphere. J. Mol. Catal. A: Chem. 2016, 414, 130-139.

(333) Podila, S.; Driss, H.; Zaman, S. F.; Ali, A. M.; Al-Zahrani, A. A.; Daous, M. A.; Petrov, L. A. Effect of Preparation Methods on the Catalyst Performance of $\mathrm{Co} / \mathrm{Mg}-\mathrm{La}$ Mixed Oxide Catalyst for $\mathrm{CO}_{\mathrm{x}}-$ Free Hydrogen Production by Ammonia Decomposition. Int. J. Hydrogen Energy 2017, 42 (38), 24213-24221.

(334) Hu, X.-C.; Wang, W.-W.; Gu, Y.-Q.; Jin, Z.; Song, Q.-S.; Jia, C.-J. Co- $\mathrm{SiO}_{2}$ Nanocomposite Catalysts for $\mathrm{CO}_{\mathrm{x}}$-Free Hydrogen Production by Ammonia Decomposition. ChemPlusChem 2017, 82 (3), 368-375.

(335) Varisli, D.; Kaykac, N. G. $\mathrm{CO}_{\mathrm{x}}$ Free Hydrogen Production over Cobalt Incorporated Silicate Structured Mesoporous Catalysts. Appl. Catal., B 2012, 127, 389-398.

(336) Varışl1, D.; Kaykac, N. G. Hydrogen from Ammonia over Cobalt Incorporated Silicate Structured Catalysts Prepared Using Different Cobalt Salts. Int. J. Hydrogen Energy 2016, 41 (14), 59555968.

(337) Lendzion-Bieluń, Z.; Narkiewicz, U.; Arabczyk, W. CobaltBased Catalysts for Ammonia Decomposition. Materials 2013, 6 (6), 2400-2409.

(338) Ji, J.; Duan, X.; Qian, G.; Zhou, X.; Tong, G.; Yuan, W. Towards an Efficient $\mathrm{CoMo} / \gamma-\mathrm{Al}_{2} \mathrm{O}_{3}$ Catalyst Using Metal Amine Metallate as an Active Phase Precursor: Enhanced Hydrogen Production by Ammonia Decomposition. Int. J. Hydrogen Energy 2014, 39 (24), 12490-12498.

(339) Duan, X.; Qian, G.; Zhou, X.; Chen, D.; Yuan, W. MCM-41 Supported Co-Mo Bimetallic Catalysts for Enhanced Hydrogen Production by Ammonia Decomposition. Chem. Eng. J. 2012, 207208, 103-108.

(340) Tagliazucca, V.; Schlichte, K.; Schüth, F.; Weidenthaler, C. Molybdenum-Based Catalysts for the Decomposition of Ammonia: In Situ X-Ray Diffraction Studies, Microstructure, and Catalytic Properties. J. Catal. 2013, 305, 277-289. 
(341) Santhana Krishnan, P.; Neelaveni, M.; Tamizhdurai, P.; Mythily, M.; Krishna Mohan, S.; Mangesh, V. L.; Shanthi, K. $\mathrm{CO}_{\mathrm{x}^{-}}$ Free Hydrogen Generation via Decomposition of Ammonia over Al, Ti and $\mathrm{Zr}$-Laponite Supported $\mathrm{MoS}_{2}$ Catalysts. Int. J. Hydrogen Energy 2020, 45 (15), 8568-8583.

(342) Xu, J.; Yan, H.; Jin, Z.; Jia, C.-J. Facile Synthesis of Stable $\mathrm{MO}_{2} \mathrm{~N}$ Nanobelts with High Catalytic Activity for Ammonia Decomposition. Chin. J. Chem. 2019, 37 (4), 364-372.

(343) Liu, H.; Wang, H.; Shen, J.; Sun, Y.; Liu, Z. Influence of Preparation Conditions on the Catalytic Performance of MoNx/SBA15 for Ammonia Decomposition. J. Nat. Gas Chem. 2006, 15 (3), 178180.

(344) Li, L.; Chu, W.; Ding, C.; Xi, X.; Jiang, R.; Yan, J. Embedded MoN@C Nanocomposites as an Advanced Catalyst for Ammonia Decomposition to $\mathrm{CO}_{\mathrm{x}}$-Free Hydrogen. Int. J. Hydrogen Energy 2017, 42 (52), 30630-30638.

(345) Wise, R. S.; Markel, E. J. Catalytic $\mathrm{NH}_{3}$ Decomposition by Topotactic Molybdenum Oxides and Nitrides: Effect on Temperature Programmed $\gamma-\mathrm{Mo}_{2} \mathrm{~N}$ Synthesis. J. Catal. 1994, 145 (2), 335-343.

(346) Tagliazucca, V.; Leoni, M.; Weidenthaler, C. Crystal Structure and Microstructural Changes of Molybdenum Nitrides Traced during Catalytic Reaction by in Situ X-Ray Diffraction Studies. Phys. Chem. Chem. Phys. 2014, 16 (13), 6182-6188.

(347) Podila, S.; Zaman, S. F.; Driss, H.; Alhamed, Y. A.; Al-Zahrani, A. A.; Petrov, L. A. Hydrogen Production by Ammonia Decomposition Using High Surface Area $\mathrm{Mo}_{2} \mathrm{~N}$ and $\mathrm{Co}_{3} \mathrm{Mo}_{3} \mathrm{~N}$ Catalysts. Catal. Sci. Technol. 2016, 6 (5), 1496-1506.

(348) Jolaoso, L. A.; Zaman, S. F.; Podila, S.; Driss, H.; Al-Zahrani, A. A.; Daous, M. A.; Petrov, L. Ammonia Decomposition over Citric Acid Induced $\gamma-\mathrm{Mo}_{2} \mathrm{~N}$ and $\mathrm{Co}_{3} \mathrm{Mo}_{3} \mathrm{~N}$ Catalysts. Int. J. Hydrogen Energy 2018, 43 (10), 4839-4844.

(349) Zaman, S. F.; Jolaloso, L. A.; Al-Zahrani, A. A.; Alhamed, Y. A.; Podila, S.; Driss, H.; Daous, M. A.; Petrov, L. A. Study of $\mathrm{Fe}_{3} \mathrm{Mo}_{3} \mathrm{~N}$ Catalyst for Ammonia Decomposition. Bulg. Chem. Commun. 2018, 50 (H), 181-188.

(350) Srifa, A.; Okura, K.; Okanishi, T.; Muroyama, H.; Matsui, T.; Eguchi, K. $\mathrm{CO}_{\mathrm{X}}$-Free Hydrogen Production via Ammonia Decomposition over Molybdenum Nitride-Based Catalysts. Catal. Sci. Technol. 2016, 6 (20), 7495-7504.

(351) Zaman, S. F.; Jolaloso, L. A.; Podila, S.; Al-Zahrani, A. A.; Alhamed, Y. A.; Driss, H.; Daous, M. M.; Petrov, L. A. Ammonia Decomposition over Citric Acid Chelated $\gamma-\mathrm{Mo}_{2} \mathrm{~N}$ and $\mathrm{Ni}_{2} \mathrm{Mo}_{3} \mathrm{~N}$ Catalysts. Int. J. Hydrogen Energy 2018, 43, 17252-17258.

(352) Choi, J.-G.; Ha, J.; Hong, J.-W. Synthesis and Catalytic Properties of Vanadium Interstitial Compounds. Appl. Catal., A 1998, $168(1), 47-56$.

(353) Choi, J.-G.; Jung, M.-K.; Choi, S.; Park, T.-K.; Kuk, I. H.; Yoo, J. H.; Park, H. S.; Lee, H.-S.; Ahn, D.-H.; Chung, H. Synthesis and Catalytic Properties of Vanadium Nitrides. Bull. Chem. Soc. Jpn. 1997, 70 (5), 993-996.

(354) Choi, J.-G.; Oh, H.-G.; Back, Y.-S. Tantalum Carbide Hydrodenitrogenation Catalysts. J. Ind. Eng. Chem. 1998, 4 (2), 94-98.

(355) Kraupner, A.; Antonietti, M.; Palkovits, R.; Schlicht, K.; Giordano, C. Mesoporous $\mathrm{Fe}_{3} \mathrm{C}$ Sponges as Magnetic Supports and as Heterogeneous Catalyst. J. Mater. Chem. 2010, 20 (29), 6019-6022.

(356) Pansare, S. S.; Torres, W.; Goodwin, J. G., Jr. Ammonia Decomposition on Tungsten Carbide. Catal. Commun. 2007, 8 (4), 649-654.

(357) Cui, X.; Li, H.; Guo, L.; He, D.; Chen, H.; Shi, J. Synthesis of Mesoporous Tungsten Carbide by an Impregnation-Compaction Route, and Its $\mathrm{NH}_{3}$ Decomposition Catalytic Activity. Dalt. Trans. 2008, No. 45, 6435-6440.

(358) Soerijanto, H.; Rödel, C.; Wild, U.; Lerch, M.; Schomäcker, R.; Schlögl, R.; Ressler, T. The Impact of Nitrogen Mobility on the Activity of Zirconium Oxynitride Catalysts for Ammonia Decomposition. J. Catal. 2007, 250 (1), 19-24.

(359) Wang, P.; Guo, J.; Xiong, Z.; Wu, G.; Wang, J.; Chen, P. The Interactions of $\mathrm{Li}_{3} \mathrm{FeN}_{2}$ with $\mathrm{H}_{2}$ and $\mathrm{NH}_{3}$. Int. J. Hydrogen Energy 2016, 41 (32), 14171-14177.
(360) Cao, H.; Guo, J.; Chang, F.; Pistidda, C.; Zhou, W.; Zhang, X.; Santoru, A.; Wu, H.; Schell, N.; Niewa, R.; Chen, P.; Klassen, T.; Dornheim, M. Transition and Alkali Metal Complex Ternary Amides for Ammonia Synthesis and Decomposition. Chem. - Eur. J. 2017, 23 (41), 9766-9771.

(361) Duan, X.; Ji, J.; Yan, X.; Qian, G.; Chen, D.; Zhou, X. Understanding Co-Mo Catalyzed Ammonia Decomposition: Influence of Calcination Atmosphere and Identification of Active Phase. ChemCatChem 2016, 8 (5), 938-945.

(362) Zhao, Z.; Zou, H.; Lin, W. Effect of Rare Earth and Other Cationic Promoters on Properties of $\mathrm{CoMoN}_{\mathrm{x}} / \mathrm{CNTs}$ Catalysts for Ammonia Decomposition. J. Rare Earths 2013, 31 (3), 247-250.

(363) Srifa, A.; Okura, K.; Okanishi, T.; Muroyama, H.; Matsui, T.; Eguchi, K. Hydrogen Production by Ammonia Decomposition over CsModified $\mathrm{Co}_{3} \mathrm{Mo}_{3} \mathrm{~N}$ Catalysts. Appl. Catal., B 2017, 218, 1-8.

(364) Leybo, D. V.; Baiguzhina, A. N.; Muratov, D. S.; Arkhipov, D. I.; Kolesnikov, E. A.; Levina, V. V.; Kosova, N. I.; Kuznetsov, D. V. Effects of Composition and Production Route on Structure and Catalytic Activity for Ammonia Decomposition Reaction of Ternary Ni-Mo Nitride Catalysts. Int. J. Hydrogen Energy 2016, 41 (6), 3854-3860.

(365) Ehiro, T.; Katagiri, K.; Yamaguchi, S.; Nishimura, T.; Saito, M.; Yoshioka, Y. The Effects of the Addition of Calcium Phosphate on Catalytic Activities for Ammonia Decomposition on CoMo-Based Catalysts. J. Ceram. Soc. Jpn. 2019, 127 (11), 802-809.

(366) Egawa, C. Ammonia Decomposition on Co/Mo(112) Model Surface. e-J. Surf. Sci. Nanotechnol. 2018, 16, 115-118.

(367) Lendzion-Bielun, Z.; Pelka, R.ł; Czekajło, Łu. Characterization of FeCo Based Catalyst for Ammonia Decomposition. The Effect of Potassium Oxide. Pol. J. Chem. Technol. 2014, 16 (4), 111-116.

(368) Lendzion-Bieluń, Z.; Arabczyk, W. Fused Fe-Co Catalysts for Hydrogen Production by Means of the Ammonia Decomposition Reaction. Catal. Today 2013, 212, 215-219.

(369) Podila, S.; Driss, H.; Zaman, S. F.; Ali, A. M.; Al-Zahrani, A. A.; Daous, M. A.; Petrov, L. A. $\mathrm{MgFe}$ and $\mathrm{Mg}-\mathrm{Co}-\mathrm{Fe}$ Mixed Oxides Derived from Hydrotalcites: Highly Efficient Catalysts for $\mathrm{CO}_{\mathrm{x}}$ Free Hydrogen Production from $\mathrm{NH}_{3}$. Int. J. Hydrogen Energy 2020, 45 (1), 873-890.

(370) Chellappa, A. S.; Fischer, C. M.; Thomson, W. J. Ammonia Decomposition Kinetics over $\mathrm{Ni}-\mathrm{Pt} / \mathrm{Al}_{2} \mathrm{O}_{3}$ for PEM Fuel Cell Applications. Appl. Catal., A 2002, 227, 231-240.

(371) Hajduk, S.; Dasireddy, V. D. B. C.; Likozar, B.; Dražić, G.; Crnjak Orel, Z. $\mathrm{CO}_{\mathrm{x}}$-Free Hydrogen Production via Decomposition of Ammonia over $\mathrm{Cu}-\mathrm{Zn}$-Based Heterogeneous Catalysts and Their Activity/Stability. Appl. Catal., B 2017, 211, 57-67.

(372) Lucentini, I.; Colli, G. G.; Luzi, C. D.; Serrano, I.; Martínez, O. M.; Llorca, J. Catalytic Ammonia Decomposition over Ni-Ru Supported on $\mathrm{CeO}_{2}$ for Hydrogen Production: Effect of Metal Loading and Kinetic Analysis. Appl. Catal., B 2021, 286, 1-10.

(373) David, W. I. F.; Makepeace, J. W.; Callear, S. K.; Hunter, H. M. A.; Taylor, J. D.; Wood, T. J.; Jones, M. O. Hydrogen Production from Ammonia Using Sodium Amide. J. Am. Chem. Soc. 2014, 136 (38), 13082-13085.

(374) Makepeace, J. W.; Hunter, H. M. A.; Wood, T. J.; Smith, R. I.; Murray, C. A.; David, W. I. F. Ammonia Decomposition Catalysis Using Lithium-Calcium Imide. Faraday Discuss. 2016, 188, 525-544.

(375) Wood, T. J.; Makepeace, J. W. Assessing Potential Supports for Lithium Amide-Imide Ammonia Decomposition Catalysts. ACS Appl. Energy Mater. 2018, 1 (6), 2657-2663.

(376) Makepeace, J. W.; Wood, T. J.; Marks, P. L.; Smith, R. I.; Murray, C. A.; David, W. I. F. Bulk Phase Behavior of Lithium ImideMetal Nitride Ammonia Decomposition Catalysts. Phys. Chem. Chem. Phys. 2018, 20 (35), 22689-22697.

(377) Wood, T. J.; Makepeace, J. W.; David, W. I. F. Neutron Diffraction and Gravimetric Study of the Manganese Nitriding Reaction under Ammonia Decomposition Conditions. Phys. Chem. Chem. Phys. 2018, 20 (13), 8547-8553.

(378) Chang, F.; Guo, J.; Wu, G.; Wang, P.; Yu, P.; Chen, P. Influence of Alkali Metal Amides on the Catalytic Activity of Manganese Nitride for Ammonia Decomposition. Catal. Today 2017, 286, 141-146. 
(379) Guo, J.; Chang, F.; Wang, P.; Hu, D.; Yu, P.; Wu, G.; Xiong, Z.; Chen, P. Highly Active $\mathrm{MnN}-\mathrm{Li}_{2} \mathrm{NH}$ Composite Catalyst for Producing $\mathrm{CO}_{\mathrm{x}}$-Free Hydrogen. ACS Catal. 2015, 5 (5), 2708-2713.

(380) Yu, P.; Guo, J.; Liu, L.; Wang, P.; Wu, G.; Chang, F.; Chen, P. Ammonia Decomposition with Manganese Nitride-Calcium Imide Composites as Efficient Catalysts. ChemSusChem 2016, 9 (4), 364369.

(381) Chiuta, S.; Everson, R. C.; Neomagus, H. W. J. P.; Le Grange, L. A.; Bessarabov, D. G. A Modelling Evaluation of an Ammonia-Fuelled Microchannel Reformer for Hydrogen Generation. Int. J. Hydrogen Energy 2014, 39 (22), 11390-11402.

(382) Tsai, W.; Weinberg, W. H. Steady-State Decomposition of Ammonia on the Ruthenium(001) Surface. J. Phys. Chem. 1987, 91 (20), 5302-5307.

(383) McCabe, R. W. Kinetics of Ammonia Decomposition on Nickel. J. Catal. 1983, 79, 445-450.

(384) Oyama, S. T. Kinetics of Ammonia Decomposition on Vanadium Nitride. J. Catal. 1992, 133, 358-369.

(385) Armenise, S.; Cazaña, F.; Monzón, A.; García-Bordejé, E. In Situ Generation of COx-Free $\mathrm{H}_{2}$ by Catalytic Ammonia Decomposition over Ru-Al-Monoliths. Fuel 2018, 233, 851-859.

(386) Armenise, S.; García-Bordejé, E.; Valverde, J. L.; Romeo, E.; Monzón, A. A Langmuir-Hinshelwood Approach to the Kinetic Modelling of Catalytic Ammonia Decomposition in an Integral Reactor. Phys. Chem. Chem. Phys. 2013, 15, 12104-12117.

(387) Abashar, M. E. E. Multi-Stage Membrane Reactors for Hydrogen Production by Ammonia Decomposition. Int. J. Petrochemistry Res. 2018, 2 (1), 109-115.

(388) Deshmukh, S. R.; Mhadeshwar, A. B.; Vlachos, D. G. Microreactor Modeling for Hydrogen Production from Ammonia Decomposition on Ruthenium. Ind. Eng. Chem. Res. 2004, 43 (12), 2986-2999.

(389) Itoh, N.; Oshima, A.; Suga, E.; Sato, T. Kinetic Enhancement of Ammonia Decomposition as a Chemical Hydrogen Carrier in Palladium Membrane Reactor. Catal. Today 2014, 236, 70-76.

(390) Kim, S.; Song, J.; Lim, H. Conceptual Feasibility Studies of a $\mathrm{CO}_{\mathrm{x}}$-Free Hydrogen Production from Ammonia Decomposition in a Membrane Reactor for PEM Fuel Cells. Korean J. Chem. Eng. 2018, 35 (3), 1-8.

(391) Zhang, Z.; Liguori, S.; Fuerst, T. F.; Way, J. D.; Wolden, C. A. Efficient Ammonia Decomposition in a Catalytic Membrane Reactor to Enable Hydrogen Storage and Utilization. ACS Sustainable Chem. Eng. 2019, 7 (6), 5975-5985.

(392) Abashar, M. E. E. Ultra-Clean Hydrogen Production by Ammonia Decomposition. J. King Saud Univ., Eng. Sci. 2018, 30, 2-11.

(393) Ganley, J. C. A Heterogeneous Chemical Reactor Analysis and Design Laboratory: The Kinetics of Ammonia Decomposition. Educ. Chem. Eng. 2017, 21, 11-16.

(394) Djéga-Mariadassou, G.; Shin, C.-H.; Bugli, G. Tamaru's Model for Ammonia Decomposition over Titanium Oxynitride. J. Mol. Catal. A: Chem. 1999, 141, 263-267.

(395) Shindo, H.; Egawa, C.; Onishi, T.; Tamaru, K. Mechanism of Ammonia Decomposition on Tungsten; $\mathrm{NH}_{3}$ and $\mathrm{ND}_{3}$ Isotope Effect. Z. Naturforsch., A: Phys. Sci. 1979, 34a (1), 96-98.

(396) Shindo, H.; Egawa, C.; Onishi, T.; Tamaru, K. Reaction Mechanism of Ammonia Decomposition on Tungsten. J. Chem. Soc., Faraday Trans. 1 1980, 76, 280-290.

(397) Tamaru, K. Adsorption Measurements during the Decomposition of Ammonia on a Tungsten Catalyst. Trans. Faraday Soc. 1961, 57, 1410-1415.

(398) Tamaru, K. A "New" General Mechanism of Ammonia Synthesis and Decomposition on Transition Metals. Acc. Chem. Res. 1988, 21 (2), 88-94.

(399) De La Osa, A. R.; De Lucas, A.; Romero, A.; Valverde, J. L.; Sánchez, P. Kinetic Models Discrimination for the High Pressure WGS Reaction over a Commercial CoMo Catalyst. Int. J. Hydrogen Energy 2011, 36 (16), 9673-9684.

(400) Wang, Y.; Kunz, M. R.; Fang, Z.; Yablonsky, G.; Fushimi, R. Accumulation Dynamics as a New Tool for Catalyst Discrimination: An
Example from Ammonia Decomposition. Ind. Eng. Chem. Res. 2019, 58 (24), 10238-10248.

(401) Logan, S. R.; Kemball, C. The Catalytic Decomposition of Ammonia on Evaporated Metal Films. Trans. Faraday Soc. 1960, 56, 144-153.

(402) Hansgen, D. A.; Vlachos, D. G.; Chen, J. G. Using First Principles to Predict Bimetallic Catalysts for the Ammonia Decomposition Reaction. Nat. Chem. 2010, 2 (6), 484-489.

(403) Hansgen, D. A.; Thomanek, L. M.; Chen, J. G.; Vlachos, D. G. Experimental and Theoretical Studies of Ammonia Decomposition Activity on Fe-Pt, Co-Pt, and Cu-Pt Bimetallic Surfaces. J. Chem. Phys. 2011, 134 (18), 184701-184707.

(404) Duan, X.; Ji, J.; Qian, G.; Fan, C.; Zhu, Y.; Zhou, X.; Chen, D.; Yuan, W. Ammonia Decomposition on Fe( $\left.\begin{array}{lll}1 & 1 & 0\end{array}\right), \mathrm{Co}\left(\begin{array}{lll}1 & 1 & 1\end{array}\right)$ and $\mathrm{Ni}\left(\begin{array}{lll}1 & 1\end{array}\right.$ 1) Surfaces: A Density Functional Theory Study. J. Mol. Catal. A: Chem. 2012, 357, 81-86.

(405) Hansgen, D. A.; Vlachos, D. G.; Chen, J. G. Ammonia Decomposition Activity on Monolayer Ni Supported on Ru, Pt and WC Substrates. Surf. Sci. 2011, 605 (23-24), 2055-2060.

(406) Zhong, J.-Q.; Zhou, X.; Yuan, K.; Wright, C. A.; Tadich, A.; Qi, D.; Li, H. X.; Wu, K.; Xu, G. Q.; Chen, W. Probing the Effect of the Pt$\mathrm{Ni}-\mathrm{Pt}(111)$ Bimetallic Surface Electronic Structures on the Ammonia Decomposition Reaction. Nanoscale 2017, 9 (2), 666-672.

(407) Love, K. S.; Emmett, P. H. The Catalytic Decomposition of Ammonia Over Iron Synthetic Ammonia Catalysts. J. Am. Chem. Soc. 1941, 63 (12), 3297-3308.

(408) Takezawa, N.; Toyoshima, I. The Change of the RateDetermining Step of the Ammonia Decomposition over an Ammonia Synthetic Iron Catalyst. J. Phys. Chem. 1966, 70 (2), 594-595.

(409) Takezawa, N.; Toyoshima, I. The Rate-Determining Step of Ammonia Decomposition over a Well-Reduced Doubly Promoted Iron Catalyst. J. Res. Inst. Catal. Hokkaido Univ. 1966, 14 (1), 41-58.

(410) Ertl, G.; Huber, M. Mechanism and Kinetics of Ammonia Decomposition on Iron. J. Catal. 1980, 61 (2), 537-539.

(411) Kunsman, C. H. The Thermal Decomposition of Ammonia on Iron Catalysts. II. J. Am. Chem. Soc. 1929, 51 (3), 688-695.

(412) Kunsman, C. H. The Thermal Decomposition of Ammonia on Tungsten, Molybdenum and Nickel. I. J. Am. Chem. Soc. 1928, 50 (8), 2100-2113.

(413) Rasim, K.; Bobeth, M.; Pompe, W.; Seriani, N. A Microkinetic Model of Ammonia Decomposition on a Pt Overlayer on $\mathrm{Au}(111)$. J. Mol. Catal. A: Chem. 2010, 325 (1-2), 15-24.

(414) Vajo, J. J.; Tsai, W.; Weinberg, W. H. Mechanistic Details of the Heterogeneous Decomposition of Ammonia on Platinum. J. Phys. Chem. 1985, 89 (15), 3243-3251.

(415) Egawa, C.; Nishida, T.; Naito, S.; Tamaru, K. Ammonia Decomposition on (1 1110$)$ and ( 001$)$ Surfaces of Ruthenium. J. Chem. Soc., Faraday Trans. 1 1984, 80 (6), 1595-1604.

(416) Huang, W.; Lai, W.; Xie, D. First-Principles Study of Decomposition of $\mathrm{NH}_{3}$ on $\operatorname{Ir}\left(\begin{array}{lll}1 & 0 & 0\end{array}\right)$. Surf. Sci. 2008, 602 (6), 12881294.

(417) Zhou, S.; Lin, S.; Guo, H. First-Principles Insights into Ammonia Decomposition Catalyzed by Ru Clusters Anchored on Carbon Nanotubes: Size Dependence and Interfacial Effects. J. Phys. Chem. C 2018, 122 (16), 9091-9100.

(418) Roy, S. K.; Ray, N.; Mukherjee, D. K.; Sen, S. P. Kinetics and Mechanism of Ammonia Decomposition over Alumina Supported Nickel Catalysts. Proceedings of Indian National Science Academy. 1975, 485-495.

(419) Tsai, W.; Vajo, J. J.; Weinberg, W. H. Inhibition by Hydrogen of the Heterogeneous Decomposition of Ammonia on Platinum. J. Phys. Chem. 1985, 89 (23), 4926-4932.

(420) Löffler, D. G.; Schmidt, L. D. Kinetics of $\mathrm{NH}_{3}$ Decomposition on Polycrystalline Pt. J. Catal. 1976, 41, 440-454.

(421) Löffler, D. G.; Schmidt, L. D. Kinetics of $\mathrm{NH}_{3}$ Decomposition on Iron at High Temperatures. J. Catal. 1976, 44, 244-258.

(422) Hashimoto, K.; Toukai, N. Decomposition of Ammonia over a Catalyst Consisting of Rutheniurn Metal and Cerium Oxides 
Supported on Y-Form Zeolite. J. Mol. Catal. A: Chem. 2000, 161 (1-2), $171-178$.

(423) Prasad, V.; Vlachos, D. G. Multiscale Model and InformaticsBased Optimal Design of Experiments: Application to the Catalytic Decomposition of Ammonia on Ruthenium. Ind. Eng. Chem. Res. 2008, 47 (17), 6555-6567.

(424) Bradford, M. C. J.; Fanning, P. E.; Vannice, M. A. Kinetics of $\mathrm{NH}_{3}$ Decomposition over Well Dispersed Ru. J. Catal. 1997, 172 (2), 479-484.

(425) Mortensen, H.; Diekhöner, L.; Baurichter, A.; Jensen, E.; Luntz, A. C. Dynamics of Ammonia Decomposition on $\mathrm{Ru}(0001)$. J. Chem. Phys. 2000, 113 (16), 6882-6887.

(426) Novell-Leruth, G.; Valcárcel, A.; Pérez-Ramírez, J.; Ricart, J. M. Ammonia Dehydrogenation over Platinum-Group Metal Surfaces. Structure, Stability, and Reactivity of Adsorbed $\mathrm{NH}_{\mathrm{x}}$ Species. J. Phys. Chem. C 2007, 111 (2), 860-868.

(427) Stolbov, S.; Rahman, T. S. First-Principles Study of Some Factors Controlling the Rate of Ammonia Decomposition on Ni and Pd Surfaces. J. Chem. Phys. 2005, 123, 204716.

(428) Guo, W.; Vlachos, D. G. Patched Bimetallic Surfaces Are Active Catalysts for Ammonia Decomposition. Nat. Commun. 2015, 6 (8619), $1-7$.

(429) Wu, H.; Sutton, J. E.; Guo, W.; Vlachos, D. G. Volcano Curves for in Silico Prediction of Mono- And Bifunctional Catalysts: Application to Ammonia Decomposition. J. Phys. Chem. C 2019, 123 (44), 27097-27104.

(430) Shustorovich, E.; Bell, A. T. Synthesis and Decomposition of Ammonia on Transition Metal Surfaces: Bond-Order-ConservationMorse-Potential Analysis. Surf. Sci. 1991, 259 (3), L791-L796.

(431) Jiang, Z.; Qin, P.; Fang, T. Theoretical Study of $\mathrm{NH}_{3}$

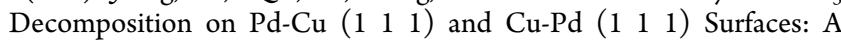
Comparison with Clean Pd ( $\left.\begin{array}{llll}1 & 1 & 1\end{array}\right)$ and $\mathrm{Cu}\left(\begin{array}{lll}1 & 1 & 1\end{array}\right)$. Appl. Surf. Sci. 2016, 371, 337-342.

(432) Mianowski, A.; Siudyga, T.; Polański, J. Szarawara-Kozik’s Temperature Criterion in the Context of Three-Parameter Equation for Modeling Ammonia or Methanol Decomposition during Heterogenous Catalysis. React. Kinet., Mech. Catal. 2018, 125 (2), 493-504.

(433) Zaman, S. F. A DFT Study of Ammonia Dissociation over $\mathrm{Mo}_{3} \mathrm{~N}_{2}$ Cluster. Bulg. Chem. Commun. 2018, $50(\mathrm{H}), 201-208$.

(434) Wood, T. J.; Makepeace, J. W.; Hunter, H. M. A.; Jones, M. O.; David, W. I. F. Isotopic Studies of the Ammonia Decomposition Reaction Mediated by Sodium Amide. Phys. Chem. Chem. Phys. 2015, 17 (35), 22999-23006.

(435) Offermans, W. K.; Jansen, A. P. J.; Van Santen, R. A.; NovellLeruth, G.; Ricart, J. M.; Pérez-Ramírez, J. Ammonia Dissociation on Pt $\{100\}$, Pt $\{111\}$, and Pt $\{211\}$ : A Comparative Density Functional Theory Study. J. Phys. Chem. C 2007, 111 (47), 17551-17557.

(436) Popa, C.; Offermans, W. K.; Van Santen, R. A.; Jansen, A. P. J. $\mathrm{Ab}$ Initio Density-Functional Theory Study of $\mathrm{NH}_{\mathrm{x}}$ Dehydrogenation and Reverse Reactions on the $\mathrm{Rh}(111)$ Surface. Phys. Rev. B: Condens. Matter Mater. Phys. 2006, 74, 155428.

(437) Gundry, P. M.; Haber, J.; Tompkins, F. C. Surface Potential Measurements on Nickel and Iron Films during the Chemisorption of Ammonia, Nitrogen, and Hydrogen. J. Catal. 1962, 1 (4), 363-371.

(438) Ertl, G.; Rüstig, J. Decomposition of $\mathrm{NH}_{3}$ on Nickel: Absence of a Magneto-Catalytic Effect. Surf. Sci. 1982, 119 (1), 314-318.

(439) Tamaru, K.; Tanaka, K.-I.; Fukasaku, S.; Ishida, S. Decomposition of Ammonia on a Nickel Catalyst. Trans. Faraday Soc. 1965, 61, 765-772.

(440) Hüttinger, M.; Küppers, J. Intermediate Product Identification for Ammonia Decomposition at Ni (110). Surf. Sci. Lett. 1983, 130 (1), L277-L282.

(441) Chrysostomou, D.; Flowers, J.; Zaera, F. Thermal Chemistry of Ammonia on $\mathrm{Ni}(110)$. Surf. Sci. 1999, 439 (1-3), 34-48.

(442) Grunze, M.; Golze, M.; Driscoll, R. K.; Dowben, P. A. Ammonia Adsorption and Decomposition on a $\mathrm{Ni}(110)$ Surface. J. Vac. Sci. Technol. 1981, 18 (2), 611-615.

(443) Bassignana, I. C.; Wagemann, K.; Küppers, J.; Ertl, G. Adsorption and Thermal Decomposition of Ammonia on a $\mathrm{Ni}(110)$
Surface: Isolation and Identification of Adsorbed $\mathrm{NH}_{2}$ and NH. Surf. Sci. 1986, 175 (1), 22-44.

(444) Duan, X.; Qian, G.; Fan, C.; Zhu, Y.; Zhou, X.; Chen, D.; Yuan, W. First-Principles Calculations of Ammonia Decomposition on $\mathrm{Ni}(110)$ Surface. Surf. Sci. 2012, 606 (3-4), 549-553.

(445) Grosman, M.; Löffler, D. G. Kinetics of Ammonia Decomposition on Polycrystalline Tungsten. J. Catal. 1983, 80 (1), 188-193.

(446) Reed, A. P. C.; Lambert, R. M. Mechanism of Ammonia Decomposition on (100) Oriented Polycrystalline Tungsten and Single-Crystal W(100). J. Phys. Chem. 1984, 88 (10), 1954-1959.

(447) Grosman, M.; Löffler, D. G. Ammonia Decomposition on Ir and Pt Wires. React. Kinet. Catal. Lett. 1987, 33 (1), 87-92.

(448) Santra, A. K.; Min, B. K.; Yi, C. W.; Luo, K.; Choudhary, T. V.; Goodman, D. W. Decomposition of $\mathrm{NH}_{3}$ on $\operatorname{Ir}(100)$ : A Temperature Programmed Desorption Study. J. Phys. Chem. B 2002, 106 (2), 340344.

(449) Amano, A.; Taylor, H. The Decomposition of Ammonia on Ruthenium, Rhodium and Palladium Catalysts Supported on Alumina. J. Am. Chem. Soc. 1954, 76 (16), 4201-4204.

(450) Zhao, J.; Cui, C.; Wang, H.; Han, J.; Zhu, X.; Ge, Q. Insights into the Mechanism of Ammonia Decomposition on Molybdenum Nitrides Based on DFT Studies. J. Phys. Chem. C 2019, 123 (1), 554564.

(451) Lanzani, G.; Laasonen, K. $\mathrm{NH}_{3}$ Adsorption and Dissociation on a Nanosized Iron Cluster. Int. J. Hydrogen Energy 2010, 35 (13), 65716577.

(452) Hellman, A.; Honkala, K.; Remediakis, I. N.; Logadóttir, Á.; Carlsson, A.; Dahl, S.; Christensen, C. H.; Nørskov, J. K. Ammonia Synthesis and Decomposition on a Ru-Based Catalyst Modeled by First-Principles. Surf. Sci. 2009, 603 (10-12), 1731-1739.

(453) Dietrich, H.; Jacobi, K.; Ertl, G. Decomposition of $\mathrm{NH}_{3}$ on $\mathrm{Ru}(1121)$. Surf. Sci. 1996, 352-354 (95), 138-141.

(454) McGill, W. J.; Sebba, F. The Kinetics of Ammonia Decomposition over Vanadium Nitride. J. Catal. 1963, 2 (2), 104-108.

(455) McGeer, J. P.; Taylor, H. S. Ammonia Decomposition and Related Phenomena on Rhenium Catalysts. J. Am. Chem. Soc. 1951, 73 (6), 2743-2751.

(456) Jiang, Z.; Qin, P.; Fang, T. Mechanism of Ammonia Decomposition on Clean and Oxygen-Covered $\mathrm{Cu}\left(\begin{array}{lll}1 & 1 & 1\end{array}\right)$ Surface: A DFT Study. Chem. Phys. 2014, 445, 59-67.

(457) Bao, J. L.; Carter, E. A. Surface-Plasmon-Induced Ammonia Decomposition on Copper: Excited-State Reaction Pathways Revealed by Embedded Correlated Wavefunction Theory. ACS Nano 2019, 13 (9), 9944-9957.

(458) Brunauer, S.; Love, K. S.; Keenan, R. G. Adsorption of Nitrogen and the Mechanism of Ammonia Decomposition Over Iron Catalysts. J. Am. Chem. Soc. 1942, 64 (4), 751-758.

(459) Bui, K. M.; Iwata, J.-I.; Kangawa, Y.; Shiraishi, K.; Shigeta, Y.; Oshiyama, A. First-Principle Study of Ammonia Decomposition and Nitrogen Incorporation on the GaN Surface in Metal Organic Vapor Phase Epitaxy. J. Cryst. Growth 2019, 507, 421-424.

(460) García-García, F. R.; Guerrero-Ruiz, A.; Rodríguez-Ramos, I.; Goguet, A.; Shekhtman, S. O.; Hardacre, C. TAP Studies of Ammonia Decomposition over Ru and Ir Catalysts. Phys. Chem. Chem. Phys. 2011, 13 (28), 12892-12899.

(461) Barbato, P. S.; Landi, G.; Lisi, L.; Di Benedetto, A. CFD Simulations of Copper-Ceria Based Microreactor for COPROX. Int. J. Chem. React. Eng. 2016, 14 (6), 1301-1313.

(462) Gyak, K.; Vishwakarma, N. K.; Hwang, Y.; Kim, J.; Yun, H.; Kim, D. 3D-Printed Monolithic SiCN Ceramic Microreactors from a Photocurable Preceramic Resin for the High Temperature Ammonia Cracking Process. React. Chem. Eng. 2019, 4, 1393-1399.

(463) Lucentini, I.; Serrano, I.; Soler, L.; Divins, N. J.; Llorca, J. Ammonia Decomposition over 3D-Printed $\mathrm{CeO}_{2}$ Structures Loaded with Ni. Appl. Catal., A 2020, 591, 117382.

(464) Ganley, J. C.; Seebauer, E. G.; Masel, R. I. Development of a Microreactor for the Production of Hydrogen from Ammonia. J. Power Sources 2004, 137 (1), 53-61. 
(465) Christian; Mitchell, M.; Kim, D.-P.; Kenis, P. J. A. Ceramic Microreactors for On-Site Hydrogen Production. J. Catal. 2006, 241 (2), 235-242.

(466) Chiuta, S.; Everson, R. C.; Neomagus, H. W. J. P.; Bessarabov, D. G. Experimental Performance Evaluation of an Ammonia-Fuelled Microchannel Reformer for Hydrogen Generation. Int. J. Hydrogen Energy 2014, 39 (14), 7225-7235.

(467) Chiuta, S.; Everson, R. C.; Neomagus, H. W. J. P.; Bessarabov, D. G. Performance Evaluation of a High-Throughput Microchannel Reactor for Ammonia Decomposition over a Commercial Ru-Based Catalyst. Int. J. Hydrogen Energy 2015, 40 (7), 2921-2926.

(468) Chiuta, S.; Everson, R. C.; Neomagus, H. W. J. P.; Bessarabov, D. G. Hydrogen Production from Ammonia Decomposition over a Commercial $\mathrm{Ru} / \mathrm{Al}_{2} \mathrm{O}_{3}$ Catalyst in a Microchannel Reactor: Experimental Validation and CFD Simulation. Int. J. Hydrogen Energy 2016, 41 (6), 3774-3785.

(469) Sørensen, R. Z.; Nielsen, L. J. E.; Jensen, S.; Hansen, O.; Johannessen, T.; Quaade, U.; Christensen, C. H. Catalytic Ammonia Decomposition: Miniaturized Production of $\mathrm{CO}_{\mathrm{x}}$-Free Hydrogen for Fuel Cells. Catal. Commun. 2005, 6 (3), 229-232.

(470) Sørensen, R. Z.; Klerke, A.; Quaade, U.; Jensen, S.; Hansen, O.; Christensen, C. H. Promoted Ru on High-Surface Area Graphite for Efficient Miniaturized Production of Hydrogen from Ammonia. Catal. Lett. 2006, 112 (1-2), 77-81.

(471) Plana, C.; Armenise, S.; Monzón, A.; García-Bordejé, E. Ni on Alumina-Coated Cordierite Monoliths for in Situ Generation of COFree $\mathrm{H}_{2}$ from Ammonia. J. Catal. 2010, 275 (2), 228-235.

(472) Armenise, S.; Roldán, L.; Marco, Y.; Monzón, A.; GarcíaBordejé, E. Elucidation of Catalyst Support Effect for $\mathrm{NH}_{3}$ Decomposition Using Ru Nanoparticles on Nitrogen-Functionalized Carbon Nanofiber Monoliths. J. Phys. Chem. C 2012, 116 (50), 2638526395.

(473) Ismagilov, Z. R.; Khairulin, S. R.; Shkrabina, R. A.; Yashnik, S. A.; Ushakov, V. A.; Moulijn, J. A.; Van Langeveld, A. D. Deactivation of Manganese Oxide-Based Honeycomb Monolith Catalyst under Reaction Conditions of Ammonia Decomposition at High Temperature. Catal. Today 2001, 69 (1-4), 253-257.

(474) Liu, Y.; Wang, H.; Li, J.; Lu, Y.; Xue, Q.; Chen, J. Microfibrous Entrapped $\mathrm{Ni} / \mathrm{Al}_{2} \mathrm{O}_{3}$ Using SS-316 Fibers for $\mathrm{H}_{2}$ Production from $\mathrm{NH}_{3}$. AIChE J. 2007, 53 (7), 1845-1849.

(475) Lu, Y.; Wang, H.; Liu, Y.; Xue, Q.; Chen, L.; He, M. Novel Microfibrous Composite Bed Reactor: High Efficiency $\mathrm{H}_{2}$ Production from $\mathrm{NH}_{3}$ with Potential for Portable Fuel Cell Power Supplies. Lab Chip 2007, 7 (1), 133-140.

(476) Deshmukh, S. R.; Vlachos, D. G. CFD Simulations of Coupled, Countercurrent Combustor/Reformer Microdevices for Hydrogen Production. Ind. Eng. Chem. Res. 2005, 44 (14), 4982-4992.

(477) Ganley, J. C.; Seebauer, E. G.; Masel, R. I. Porous Anodic Alumina Microreactors for Production of Hydrogen from Ammonia. AIChE J. 2004, 50 (4), 829-834.

(478) Dillon, A. C.; Gupta, P.; Robinson, M. B.; Bracker, A. S.; George, S. M. FTIR Studies of Water and Ammonia Decomposition on Silicon Surfaces. J. Electron Spectrosc. Relat. Phenom. 1990, 54-55 (C), 1085-1095.

(479) Plana, C.; Armenise, S.; Monzón, A.; García-Bordejé, E. Process Optimisation of in Situ $\mathrm{H}_{2}$ Generation from Ammonia Using $\mathrm{Ni}$ on Alumina Coated Cordierite Monoliths. Top. Catal. 2011, 54 (13-15), 914-921.

(480) Wang, M.; Li, J.; Chen, L.; Lu, Y. Miniature $\mathrm{NH}_{3}$ Cracker Based on Microfibrous Entrapped $\mathrm{Ni}-\mathrm{CeO}_{2} / \mathrm{Al}_{2} \mathrm{O}_{3}$ Catalyst Monolith for Portable Fuel Cell Power Supplies. Int. J. Hydrogen Energy 2009, 34 (4), $1710-1716$

(481) Zou, J.; Lu, D.-P.; Zhai, Q.-J. The Research on Ni-Based Ammonia Decomposition Catalyst. Appl. Mech. Mater. 2014, 644-650, $5364-5367$

(482) Pereira, S. I. Modeling of Fixed Bed Catalytic Reactors. Comput. Chem. Eng. 1985, 1354 (December 2017), 535 DOI: 10.1016/00981354(85)80028-4.
(483) Chen, J.; Yang, H.; Wang, N.; Ring, Z.; Dabros, T. Mathematical Modeling of Monolith Catalysts and Reactors for Gas Phase Reactions. Appl. Catal., A 2008, 345 (1), 1-11.

(484) Chen, L.; Kang, Q.; He, Y.-L.; Tao, W.-Q. Pore-Scale Simulation of Coupled Multiple Physicochemical Thermal Processes in Micro Reactor for Hydrogen Production Using Lattice Boltzmann Method. Int. J. Hydrogen Energy 2012, 37 (19), 13943-13957.

(485) Molaeimanesh, G. R.; Sanati Davarani, M. H. A Pore-Scale Model for Microfibrous Ammonia Cracking Microreactors via Lattice Boltzmann Method. Korean J. Chem. Eng. 2016, 33 (4), 1211-1219.

(486) Waghode, A. N.; Hanspal, N. S.; Shigidi, I. M. T. A.; Nassehi, V.; Hellgardt, K. Computer Modelling and Numerical Analysis of Hydrodynamics and Heat Transfer in Non-Porous Catalytic Reactor for the Decomposition of Ammonia. Chem. Eng. Sci. 2005, 60 (21), $5862-5877$

(487) Qazi Zade, A.; Renksizbulut, M.; Friedman, J. Ammonia Decomposition for Hydrogen Production in Catalytic Microchannels with Slip/Jump Effects. J. Appl. Fluid Mech. 2015, 8 (4), 703-712.

(488) Badescu, V. Optimal Design and Operation of Ammonia Decomposition Reactors. Int. J. Energy Res. 2020, 44, 5360-5384.

(489) Chein, R.-Y.; Chen, Y.-C.; Chang, C.-S.; Chung, J. N. Numerical Modeling of Hydrogen Production from Ammonia Decomposition for Fuel Cell Applications. Int. J. Hydrogen Energy 2010, 35 (2), 589-597.

(490) Alagharu, V.; Palanki, S.; West, K. N. Analysis of Ammonia Decomposition Reactor to Generate Hydrogen for Fuel Cell Applications. J. Power Sources 2010, 195 (3), 829-833.

(491) Gallucci, F.; Fernandez, E.; Corengia, P.; van Sint Annaland, M. Recent Advances on Membranes and Membrane Reactors for Hydrogen Production. Chem. Eng. Sci. 2013, 92, 40-66.

(492) López, E.; Divins, N. J.; Llorca, J. Hydrogen Production from Ethanol over $\mathrm{Pd}-\mathrm{Rh} / \mathrm{CeO}_{2}$ with a Metallic Membrane Reactor. Catal. Today 2012, 193 (1), 145-150.

(493) Rahimpour, M. R.; Asgari, A. Production of Hydrogen from Purge Gases of Ammonia Plants in a Catalytic Hydrogen-Permselective Membrane Reactor. Int. J. Hydrogen Energy 2009, 34 (14), 5795-5802.

(494) Gómez-García, M. Á.; Dobrosz-Gómez, I.; Fontalvo, J.; Rynkowski, J. M. Membrane Reactor Design Guidelines for Ammonia Decomposition. Catal. Today 2012, 191 (1), 165-168.

(495) Hedayati, A.; Llorca, J. Experimental Study of 2-Methoxyethanol Steam Reforming in a Membrane Reactor for Pure Hydrogen Production. Fuel 2017, 190, 312-317.

(496) Palo, E.; Salladini, A.; Morico, B.; Palma, V.; Ricca, A.; Iaquaniello, G. Application of Pd-Based Membrane Reactors: An Industrial Perspective. Membranes (Basel, Switz.) 2018, 8 (101), 1-15.

(497) Lundin, S.-T. B.; Yamaguchi, T.; Wolden, C. A.; Oyama, S. T.; Way, J. D. The Role (or Lack Thereof) of Nitrogen or Ammonia Adsorption-Induced Hydrogen Flux Inhibition on Palladium Membrane Performance. J. Membr. Sci. 2016, 514, 65-72.

(498) Sakamoto, F.; Kinari, Y.; Chen, F. L.; Sakamoto, Y. Hydrogen Permeation through Palladium Alloy Membranes in Mixture Gases of $10 \%$ Nitrogen and Ammonia in the Hydrogen. Int. J. Hydrogen Energy 1997, 22 (4), 369-375.

(499) Liu, J.; Ju, X.; Tang, C.; Liu, L.; Li, H.; Chen, P. High Performance Stainless-Steel Supported Pd Membranes with a Fingerlike and Gap Structure and Its Application in $\mathrm{NH}_{3}$ Decomposition Membrane Reactor. Chem. Eng. J. 2020, 388, 124245.

(500) Li, G.; Kanezashi, M.; Lee, H. R.; Maeda, M.; Yoshioka, T.; Tsuru, T. Preparation of a Novel Bimodal Catalytic Membrane Reactor and Its Application to Ammonia Decomposition for $\mathrm{CO}_{\mathrm{x}}$-Free Hydrogen Production. Int. J. Hydrogen Energy 2012, 37 (17), 1210512113.

(501) Li, G.; Kanezashi, M.; Tsuru, T. Highly Enhanced Amsmonia Decomposition in a Bimodal Catalytic Membrane Reactor for $\mathrm{CO}_{\mathrm{x}}$ Free Hydrogen Production. Catal. Commun. 2011, 15 (1), 60-63.

(502) Li, G.; Kanezashi, M.; Yoshioka, T.; Tsuru, T. Ammonia Decomposition in Catalytic Membrane Reactors: Simulation and Experimental Studies. AIChE J. 2013, 59 (1), 168-179.

(503) Cheng, H.; Meng, B.; Li, C.; Wang, X.; Meng, X.; Sunarso, J.; Tan, X.; Liu, S. Single-Step Synthesized Dual-Layer Hollow Fiber 
Membrane Reactor for on-Site Hydrogen Production through Ammonia Decomposition. Int. J. Hydrogen Energy 2020, 45 (12), $7423-7432$.

(504) Zhang, J.; Xu, H.; Li, W. High-Purity $\mathrm{CO}_{\mathrm{x}}$-Free $\mathrm{H}_{2}$ Generation from $\mathrm{NH}_{3}$ via the Ultra Permeable and Highly Selective Pd Membranes. J. Membr. Sci. 2006, 277 (1-2), 85-93.

(505) García-García, F. R.; Ma, Y. H.; Rodríguez-Ramos, I.; GuerreroRuiz, A. High Purity Hydrogen Production by Low Temperature Catalytic Ammonia Decomposition in a Multifunctional Membrane Reactor. Catal. Commun. 2008, 9 (3), 482-486.

(506) Collins, J. P.; Way, J. D. Catalytic Decomposition of Ammonia in a Membrane Reactor. J. Membr. Sci. 1994, 96 (3), 259-274.

(507) Rizzuto, E.; Palange, P.; Del Prete, Z. Characterization of an Ammonia Decomposition Process by Means of a Multifunctional Catalytic Membrane Reactor. Int. J. Hydrogen Energy 2014, 39 (22), 11403-11410.

(508) Itoh, N.; Kikuchi, Y.; Furusawa, T.; Sato, T. Tube-Wall Catalytic Membrane Reactor for Hydrogen Production by Low-Temperature Ammonia Decomposition. Int. J. Hydrogen Energy 2020, DOI: 10.1016/ j.ijhydene.2020.03.162.

(509) Israni, S. H.; Nair, B. K. R.; Harold, M. P. Hydrogen Generation and Purification in a Composite Pd Hollow Fiber Membrane Reactor: Experiments and Modeling. Catal. Today 2009, 139 (4), 299-311.

(510) Prasad, V.; Karim, A. M.; Ulissi, Z.; Zagrobelny, M.; Vlachos, D. G. High Throughput Multiscale Modeling for Design of Experiments, Catalysts, and Reactors: Application to Hydrogen Production from Ammonia. Chem. Eng. Sci. 2010, 65 (1), 240-246.

(511) Di Carlo, A.; Dell'Era, A.; Del Prete, Z. 3D Simulation of Hydrogen Production by Ammonia Decomposition in a Catalytic Membrane Reactor. Int. J. Hydrogen Energy 2011, 36 (18), 1181511824.

(512) Cechetto, V.; Di Felice, L.; Medrano, J. A.; Makhloufi, C.; Zuniga, J.; Gallucci, F. $\mathrm{H}_{2}$ Production via Ammonia Decomposition in a Catalytic Membrane Reactor. Fuel Process. Technol. 2021, 216, 106772. (513) Shwe Hla, S.; Dolan, M. D. CFD Modelling of a Membrane Reactor for Hydrogen Production from Ammonia. IOP Conf. Ser.: Mater. Sci. Eng. 2018, 297, 012027.

(514) Divins, N. J.; Angurell, I.; Escudero, C.; Pérez-Dieste, V.; Llorca, $\mathrm{J}$. Influence of the support on surface rearrangements of bimetallic nanoparticles in real catalysts. Science 2014, 346, 620-623. 